\title{
The XMM-Newton survey in the Marano field
}

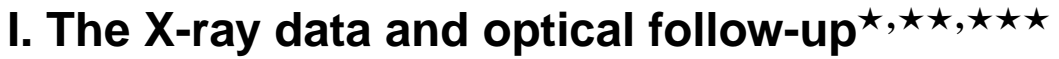

\author{
M. Krumpe ${ }^{1}$, G. Lamer ${ }^{1}$, A. D. Schwope ${ }^{1}$, S. Wagner ${ }^{2}$, G. Zamorani ${ }^{3}$, M. Mignoli ${ }^{3}$, R. Staubert ${ }^{4}$, \\ L. Wisotzki ${ }^{1}$, and G. Hasinger ${ }^{5}$
}

1 Astrophysikalisches Institut Potsdam, An der Sternwarte 16, 14482 Potsdam, Germany e-mail: mkrumpe@aip.de

2 Landessternwarte Heidelberg-Königstuhl, 69117 Heidelberg, Germany

3 INAF - Osservatorio Astronomico di Bologna, Bologna, Italy

${ }^{4}$ Universität Tübingen, Institut für Astronomie und Astrophysik, 72076 Tübingen, Germany

5 Max-Planck-Institut für Extraterrestrische Physik, Giessenbachstrasse, Postfach 1312, 85741 Garching, Germany

Received 16 June 2006 / Accepted 7 December 2006

\begin{abstract}
We report on a medium deep XMM-Newton survey of the Marano Field and optical follow-up observations. The mosaicked XMM-Newton pointings in this optical quasar survey field cover $0.6 \mathrm{deg}^{2}$ with a total of $120 \mathrm{ks}$ good observation time. We detected $328 \mathrm{X}$-ray sources in total. The turnover flux of our sample is $f_{\mathrm{X}} \sim 5 \times 10^{-15} \mathrm{erg} \mathrm{cm}^{-2} \mathrm{~s}^{-1}$ in the $0.2-10 \mathrm{keV}$ band. With VLT FORS1 and FORS2 spectroscopy we classified 96 new X-ray counterparts.

The central $0.28 \mathrm{deg}^{2}$, where detailed optical follow-up observations were performed, contain $170 \mathrm{X}$-ray sources (detection likelihood $M L>10$ ), out of which 48 had already been detected by ROSAT. In this region we recover 23 out of 29 optically selected quasars. With a total of 110 classifications in our core sample we reach a completeness of $\sim 65 \%$. About one-third of the XMM-Newton sources are classified as type II AGN with redshifts mostly below 1.0. Furthermore, we detect five high redshift type II AGN $(2.2 \leq z \leq 2.8)$. We show that the true redshift distribution of type II AGN remains uncertain, since their lack of emission lines in a wide optical wavelength range hampers their identification in the redshift range $1<z<2$. The optical and X-ray colours of the core sample indicate that most of the still unidentified X-ray sources are likely to be type II AGN. We calculate absorbing column densities and show that the ratio of absorbed to unabsorbed objects is significantly higher for type II AGN than for type I AGN. Nevertheless, we find a few unabsorbed type II AGN. The X-ray hardness ratios of some high redshift type I AGN also give an indication of heavy absorption. However, none of these type I objects is bright enough for spectral extraction and detailed model fitting. Types I and II AGN cover the same range in intrinsic X-ray luminosity, $\left(10^{43} \leq L_{\mathrm{X}} \leq 10^{46}\right)$, although type II AGN have a lower median intrinsic X-ray luminosity $\left(\log L_{\mathrm{X}} \sim 44.0\right)$ compared to type I AGN $\left(\log L_{\mathrm{X}} \sim 44.4\right)$.

Furthermore, we classified three X-ray bright optically normal galaxies (XBONGs) as counterparts. They show properties similar to type II AGN, probably harbouring an active nucleus.
\end{abstract}

Key words. surveys - X-rays: galaxies - galaxies: active - galaxies: quasars: general

\section{Introduction}

X-ray surveys are essential to characterise the source population of the X-ray sky and are the only means to understand the nature of the X-ray background radiation. Deep X-ray surveys had been carried out during the last decades (Hasinger et al. 1998; Lehmann et al. 2001; Alexander et al. 2003) and showed that up to $80 \%$ of the soft X-ray background is due to active galactic nuclei (AGN). XMM-Newton and CHANDRA, thanks to their high sensitivity at hard X-rays, opened the absorbed X-ray universe for further studies and revealed a large population of

* Based on observations obtained at the European Southern Observatory, Paranal, Chile (ESO programmes 66.B-0127(A) and 70.A-0651(A) and on observations obtained with XMM-Newton, an ESA science mission with instruments and contributions directly funded by ESA Member States and NASA.

$\star \star$ Appendices A-D are only available in electronic form at http: //www . aanda.org

$\star \star \star$ Table 8 is also available in electronic form at the CDS via anonymous ftp to cdsarc.u-strasbg.fr $(130.79 .128 .5)$ or via http://cdsweb.u-strasbg.fr/cgi-bin/qcat?]/A+A/466/41 obscured, low-luminosity, low-redshift X-ray sources (Hasinger et al. 2001). To characterise the X-ray sky properly and understand the X-ray background peak at $\sim 30 \mathrm{keV}$ (Worsley et al. 2005), numerous surveys with a large variety in limiting flux and survey area are needed (see Fig. 1, in Brandt \& Hasinger 2005). In this paper, we present new XMM-Newton data and spectroscopic classifications of X-ray sources in the Marano Field.

The Marano Field was named by an early optical quasar survey up to a limiting magnitude of $B_{\mathrm{J}}=22.0$ by Marano et al. (1988). Based on different optical selection techniques (colour-colour diagrams, grism plates, variability analysis) they discovered 23 broad emission line quasars and defined an extensive list of quasar candidates. Zitelli et al. (1992) completed this work by presenting a spectroscopically complete sample of quasars with $B_{\mathrm{J}} \leq 22.0$ using this list of quasar candidates. They confirmed 54 quasars including the former 23 quasars. Between December 1992 and July 1993, ROSAT observed the central Marano Field $\left(\sim 0.2 \mathrm{deg}^{2}\right)$ for $56 \mathrm{ks}$ (Zamorani et al. 1999). The data revealed 50 X-ray sources with a limiting 
Table 1. XMM-Newton observations of the Marano Field. filter: $T$.thin, M.-medium.

\begin{tabular}{ccccrc}
\hline \hline $\begin{array}{c}\text { XMM-orbit/ } \\
\text { ObsID }\end{array}$ & $\begin{array}{c}\text { Date } \\
(2000)\end{array}$ & $\begin{array}{c}\text { RA } \\
\text { (3h:min:ss) }\end{array}$ & $\begin{array}{c}\text { Dec } \\
(-55: m i n: s s)\end{array}$ & $\begin{array}{r}\text { Time } \\
\text { (ks) }\end{array}$ & Filter \\
\hline $106 / 0112940201$ & $7 / 7$ & $15: 27$ & $06: 13$ & 10.3 & $T$. \\
$107 / 0112940201$ & $10 / 7$ & $16: 03$ & $21: 41$ & 10.4 & $T$. \\
$107 / 0112940301$ & $10 / 7$ & $15: 27$ & $11: 22$ & 10.6 & $T$. \\
$107 / 0112940401$ & $10 / 7$ & $15: 27$ & $16: 32$ & 10.5 & $T$. \\
$107 / 0112940501$ & $10 / 7$ & $15: 27$ & $21: 41$ & 7.8 & $T$. \\
$129 / 0129321001$ & $22 / 8$ & $16: 03$ & $11: 22$ & 6.1 & $T$. \\
$129 / 0129320801$ & $22 / 8$ & $16: 03$ & $16: 32$ & 10.6 & $M$. \\
$129 / 0129320901$ & $22 / 8$ & $16: 03$ & $06: 13$ & 10.6 & $T$. \\
$130 / 0110970101$ & $24 / 8$ & $13: 09$ & $03: 54$ & 10.9 & $T$. \\
$130 / 0110970201$ & $24 / 8$ & $14: 15$ & $11: 22$ & 9.1 & $T$. \\
$130 / 0110970301$ & $24 / 8$ & $14: 15$ & $16: 32$ & 9.1 & $T$. \\
$130 / 0110970401$ & $24 / 8$ & $14: 15$ & $21: 41$ & 9.1 & $T$. \\
$131 / 0110970501$ & $26 / 8$ & $14: 51$ & $06: 13$ & 9.1 & $T$. \\
$131 / 0110970601$ & $26 / 8$ & $14: 51$ & $11: 22$ & 9.9 & $T$. \\
$133 / 0110970701$ & $30 / 8$ & $14: 51$ & $16: 32$ & 9.9 & $T$. \\
$133 / 0110970801$ & $30 / 8$ & $14: 51$ & $21: 41$ & 8.8 & $T$. \\
\hline
\end{tabular}

flux of $f_{\mathrm{X}} \geq 3.7 \times 10^{-15} \mathrm{erg} \mathrm{cm}^{-2} \mathrm{~s}^{-1}$ in the ROSAT band $(0.5-$ $2.0 \mathrm{keV})$. Multi-colour CCD and spectroscopic data identified $42 \mathrm{X}$-ray sources (33 quasars, 2 galaxies, 3 clusters, and 4 stars). $66 \%$ of the optically selected quasars within the ROSAT fieldof-view were detected as ROSAT X-ray sources. Gruppioni et al. (1999) carried out a deep radio survey at 1.4 and $2.4 \mathrm{GHz}$ and detected 68 radio sources $\left(f_{\mathrm{R}}>0.2 \mathrm{mJy}\right)$. Follow-up observation provided redshifts for 30 objects.

Our new XMM-Newton data comprise an area three times larger, compared to the ROSAT survey, and are thus almost comparable in size to the optical quasar survey. We are reaching a survey sensitivity of $f_{\mathrm{X}} \sim 5 \times 10^{-15} \mathrm{erg} \mathrm{cm}^{-2} \mathrm{~s}^{-1}$ (turnover flux) over a contiguous area of $0.6 \mathrm{deg}^{2}$. The XMM-Newton survey of the Marano Field is thus comparable with deep ROSAT surveys (e.g., the UDS, Hasinger et al. 1998) or with medium deep CHANDRA surveys (e.g., ChaMP, Green et al. 2004).

The paper is organised as follows. In Sect. 2 we list the X-ray and optical data and the reduction of the data. Section 3 describes and summarises the spectroscopic classification of the $\mathrm{X}$-ray sources. In Sect. 4 we make use of the spectroscopic classification and redshift determination to analyse the properties of different object classes. In this section we concentrate on a "core sample" of objects in the central part of the field, where we reached the highest degree of completeness in the spectroscopic classification. Section 5 addresses additional objects in the Marano Field that are not X-ray detected. These objects were obtained as a control sample. Section 6 dicusses the results of the different object classes. Finally, our conclusions are outlined in Sect. 7. Unless mentioned otherwise, all errors refer to a $68 \%$ confidence interval.

\section{Observations and data reduction}

\subsection{XMM-Newton X-ray observations}

Since the area of the optical quasar survey in the Marano Field is larger than the XMM-Newton field of view, the X-ray observations have been performed as a grid of $4 \times 4$ overlapping pointings with a spacing of $\sim 5$ arcmin in right ascension and declination. The pointing in the north-western corner of the grid was shifted to cover the position of a deep far-infrared survey with the ISO satellite (Table 1 and Fig. 1). The ISO data are not addressed in this paper.

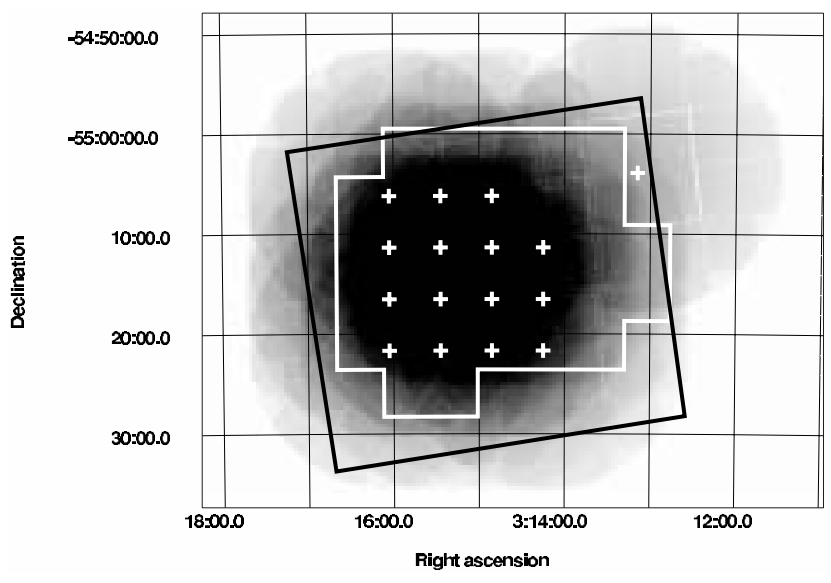

Fig. 1. Averaged mos 1 and mos 2 exposure map (0.5-2.0 keV) of the $4 \times 4$ pointing pattern (small white crosses). The exposure time in the central region reaches $78 \mathrm{ks}$. The black rectangle shows the area covered by the WFI $R$-band images. The white polygon marks the area covered by the SOFI $K$-band images (see Sect. 2.2).

Due to the overlapping pointings some deviations from the standard XMM-Newton data analysis procedures were necessary, and are described here. The photon event tables from the 16 grid pointings were merged into a single event table with a common sky coordinate frame using the XMM SAS merge task. In this coordinate frame we created 240 images (one for each pointing, energy range, and instrument). The 5 bands used are 0.2-0.5 keV, 0.5-2.0 keV, 2.0-4.5 keV, 4.5-7.5 keV, and 7.5-12.0 keV. Each image has $963 \times 963$ pixels with four arcseconds binning. For each of these images separate exposure maps and background maps were created using the XMM SAS tasks eexpmap and esplinemap. One of the pointings (ObsID 0129320801) had accidentally been scheduled with "medium" thickness filters, while all other pointings had been observed with "thin" filters. To correct for the somewhat lower throughput of the medium filters at soft energies, we multiplied all exposure maps of this pointing with a correction factor derived from the energy conversion factors for the "thin" and "medium" filters. These ECFs were taken from the SSC document SSC-LUX-TN-0059 v.3 (Osborne 2001). The correction is largest in the softest band $(0.2-0.5 \mathrm{keV})$, where the effective exposure in the affected pointing is reduced by $11 \%$.

We then added the images, exposure maps, and background maps of the individual pointings, resulting in 15 images for 5 energy bands and 3 detectors. These images were used simultaneously as input for a detection run with eboxdetect and emldetect. The task emldetect applies a PSF-fit to each source found by eboxdetect to determine the source parameters. Since we use merged mosaic images of the field, each source image results from the superposition of several pointings. In each pointing the source is detected at a different position on the detector. Therefore, the standard configuration of emldetect, which uses the off-axis angle and position-angle of each source to extract the appropriate PSF from calibration files, could not be used here. Instead emldetect was modified to use the calibration PSF corresponding to an off-axis angle $\theta=5$ arcmin throughout the entire field. This PSF is circular symmetric and is a good representation of the point sources in the merged images. For this work no extent models were fitted to the sources, therefore the source list produced by emldetect does not contain any information whether a source is extended or point-like. Generally, the X-ray data reduction and analysis 
was performed with XMM SAS version 5.1 (from 18/06/2001) with the abovementioned exception of an adapted version of emldetect. The version of emldetect used here suffered from an error, which resulted in the overestimation of detection likelihood values (XMM-Newton-NEWS \#29, 11-Mar.-2003). All likelihood values quoted here have been corrected to adhere to the relation $M L=-\ln (P)$, where $M L$ is the detection likelihood and $P$ is the probability that the detection is caused by a random fluctuation of the background. All count rates and derived quantities are taken from the PSF fitting of emldetect. Using the combined expoure maps, emldetect corrects the source count rates for all spatial variations of telescope and detector efficiency, i.e., the count rates relate to the optical axis of each EPIC camera. Following Cash (1979), the $68 \%$ confidence intervals for the source positions and source fluxes were calculated using emldetect as follows: each parameter is varied until the statistic

$C=2 \sum_{i=1}^{N}\left(e_{i}-n_{i} \ln e_{i}\right)$

exceeds the best-fit value of $C$ by 1 , where $e_{i}$ is the source model at the position of pixel $i$ and $n_{i}$ is the number of counts in pixel $i$. We used energy conversion factors (ECFs) to calculate source fluxes in the band $0.2-10 \mathrm{keV}$ (from the total EPIC count rate in the band $0.2-12 \mathrm{keV}$ ) and in the band $0.5-2.0 \mathrm{keV}$ (from the EPIC count rate in the band $0.5-2.0 \mathrm{keV}$ ). The ECFs were derived by using XSPEC and the XMM SAS calibration files to simulate power-law spectra with photon index $\Gamma=1.8$ and the galactic column density of the field $N_{\mathrm{H}}=2.7 \times 10^{20} \mathrm{~cm}^{-2}$.

For all sources 3 hardness ratios of the form $H R=\left(C R_{2}-C R_{1}\right) /\left(C R_{1}+C R_{2}\right)$ were calculated between the adjacent pn-detector energy bands $0.2-0.5 \mathrm{keV}, 0.5-2.0 \mathrm{keV}$, $2.0-4.5 \mathrm{keV}$, and $4.5-7.5 \mathrm{keV}$. For example, HR2 was calculated between the bands $0.5-2.0 \mathrm{keV}$ and $2.0-4.5 \mathrm{keV}$. The different energy responses of the mos- and pn-detectors result in different hardness ratios for the same source. If not noted otherwise the pn-values are used throughout the paper.

In total we detected $328 \mathrm{X}$-ray sources with detection likelihoods $M L \geq 5.0$. The $\mathrm{X}$-ray fluxes are in the range $f_{\mathrm{X}}=(0.16-54) \times 10^{-14} \mathrm{erg} \mathrm{cm}^{-2} \mathrm{~s}^{-1}(0.2-10 \mathrm{keV})$. The X-ray flux histogram (Fig. 3) shows the X-ray detection limit of our survey. Below $f_{\mathrm{X}} \sim 5 \times 10^{-15} \mathrm{erg} \mathrm{cm}^{-2} \mathrm{~s}^{-1}$ we are unable to detect the majority of the X-ray sources. In the central region (Fig. 2) we found $252 \mathrm{X}$-ray sources. The complete X-ray source list can be found in the Online Material, Appendix A.

Since the PSF-modelling task emldetect was only used with point-source models here, the detection procedure was not very sensitive to diffuse emission from clusters of galaxies. However, visual inspection of the X-ray images revealed no obviously extended sources. We estimate that extended cluster emission down to $0.5-2.0 \mathrm{keV}$ fluxes of $2 \times 10^{-14} \mathrm{erg} \mathrm{cm}^{-2} \mathrm{~s}^{-1}$ should be detectable in this survey. The cluster $\log N(\log S)$ of Rosati et al. (1998) gives a surface density of $\sim 6$ clusters per square degree at this flux. We would therefore expect about four clusters in the total area of the XMM observations and about two clusters in the $0.28 \mathrm{deg}^{2}$ core region of the survey. Given the considerable cosmic variance of the cluster surface density the non-detection of clusters in the survey is consistent with the $\log N(\log S)$. In any case the small number of expected clusters does not suggest a significant contribution of clusters of galaxies to our source sample.

XMM-Newton redetected all 50 ROSAT X-ray sources (Zamorani et al. 1999). However, two ROSAT X-ray sources

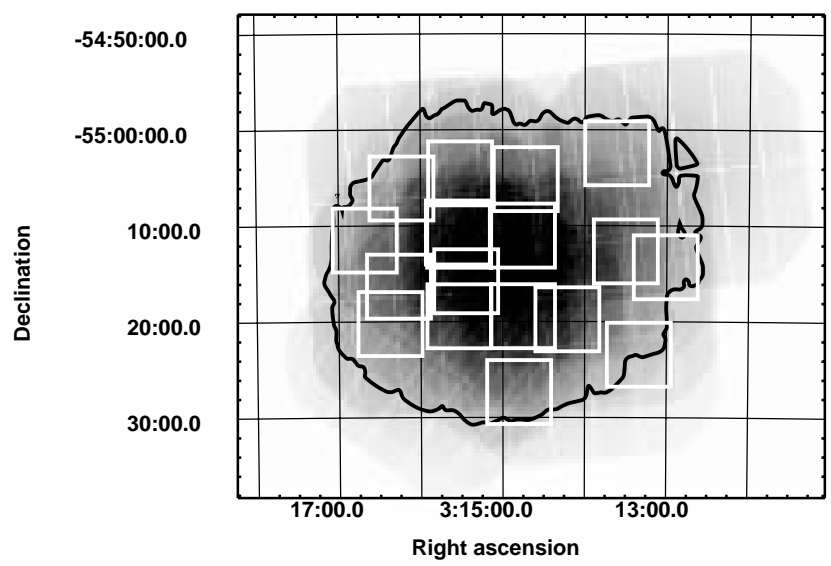

Fig. 2. PN exposure map $(0.5-2.0 \mathrm{keV})$ of the $4 \times 4$ pointing pattern. The exposure time in the central region reaches $35.5 \mathrm{ks}$. The area inside the black contour markes the "core sample" region where the pndetector exposure time exceeds six ks. White rectangles show the position of the spectroscopic masks for the optical follow-up observations (see Sect. 2.4).

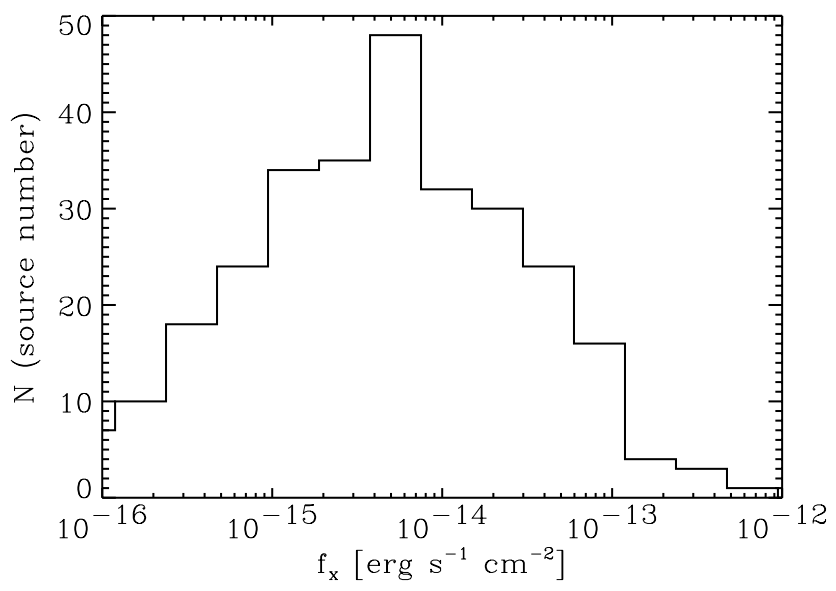

Fig. 3. $0.2-10 \mathrm{keV} \mathrm{X}$-ray flux histogram of the $328 \mathrm{XMM}-$ Newton detected X-ray sources in the Marano Field. Up to an X-ray flux of $f_{\mathrm{X}} \sim 5 \times 10^{-15} \mathrm{erg} \mathrm{cm}^{-2} \mathrm{~s}^{-1}$ (turnover flux) we detect more and more sources. Below that X-ray flux the detection rate decreases dramactically.

have low detection likelihoods and were thus not included in the final XMM-Newton X-ray source list (Online Material, Appendix A). Figure 4 compares ROSAT and EPIC X-ray fluxes in the $0.5-2.0 \mathrm{keV}$ band.

The X-ray fluxes of both missions are comparable down to ROSAT fluxes of $f_{\mathrm{X}} \sim 6.0 \times 10^{-15} \mathrm{erg} \mathrm{cm}^{-2} \mathrm{~s}^{-1}$. Among the 13 objects (broad emission line objects) with $f_{\mathrm{X}} \geq 10^{-14} \mathrm{erg} \mathrm{cm}^{-2} \mathrm{~s}^{-1}$ (ROSAT), five show variability of about a factor of two in both directions. Throughout the whole paper we refer to the X-ray sources by mentioning the X-ray source number without any letter as suffix (e.g. 452). These numbers refer to the sequence numbers in the original emldetect source list and are non-contiguous due to the removal of sources below $M L=5.0$ after correction of the detection likelihoods.

\subsection{The optical data}

\subsubsection{WFI $R$-band observations}

The central region of the Marano Field has been observed in the $U B V R I$ bands with the ESO Wide-Field-Imager (WFI). Here 


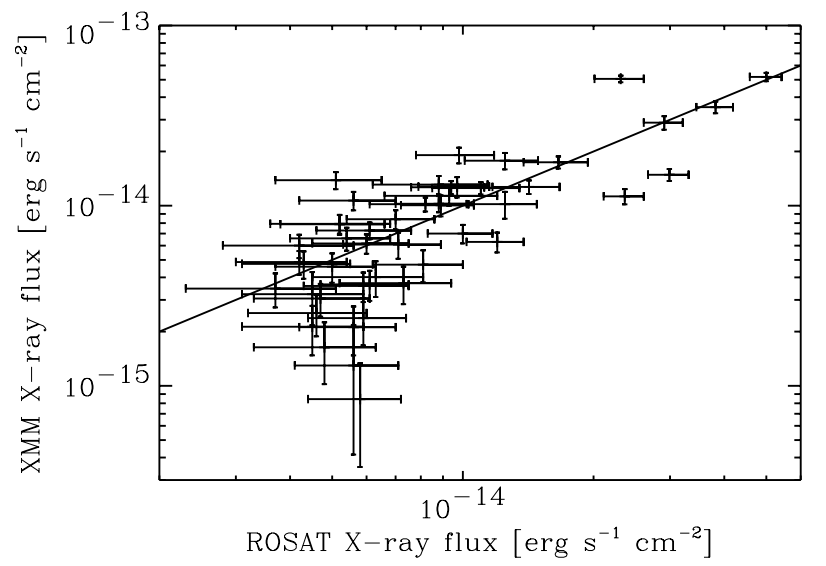

Fig. 4. Comparison between the $0.5-2 \mathrm{keV} X$-ray fluxes measured by ROSAT and XMM-Newton of all 50 ROSAT X-ray sources in the Marano Field. XMM-Newton fluxes are averages of the three individual EPIC camera fluxes. The black solid line represents equal ROSAT and XMM-Newton fluxes.

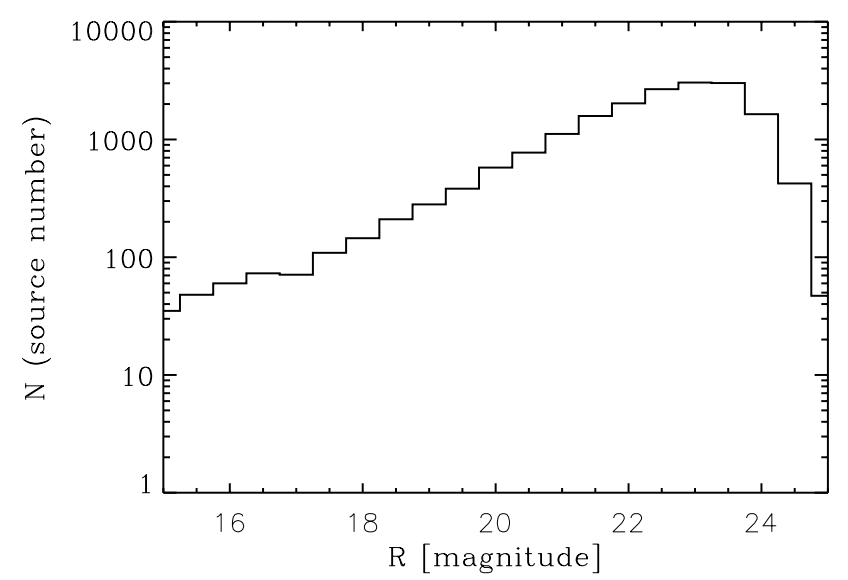

Fig. 5. Magnitude histogram of the sources detected in the WFI $R$-band image. Completeness in detecting objects is lost for $R>23$.

we make use of the deep (7000 s) $R$-band image (Mignoli $\&$ Zamorani 1998) with a limiting magnitude $R \simeq 22.5$ (defined as turnover magnitude minus $0.5 \mathrm{mag}$, see Fig. 5). The WFI-image covers an area of $35 \times 32$ arcmin well aligned with the region of the deepest XMM-Newton coverage (Fig. 1).

\subsubsection{SOFI $K$-band observations}

The $K$-band data $(2.0-2.3 \mu \mathrm{m})$ were obtained at the ESO New Technology Telescope (NTT) with the SOFI instrument. With an area of $754 \operatorname{arcmin}^{2}$ the $K$-band mosaic covers a slightly smaller area than the WFI $R$-band observation and is well aligned with the deepest XMM-Newton exposure (Fig. 1). The SOFI observations consist of a mosaic of 33 jittered pointings, each covering $5 \times 5$ arcmin with an exposure time of 29 min each. The limiting magnitude is $K \simeq 20$ (turnover magnitude minus $0.5 \mathrm{mag}$, see Fig. 6).

\subsubsection{VLT $R$-band pre-images}

$R$-band pre-images for the spectroscopic follow-up observation were obtained with the Focal Reducer and Spectrograph FORS1 and FORS2 at the ESO Very Large Telescope (VLT). The first run (FORS1) contained six and the second run

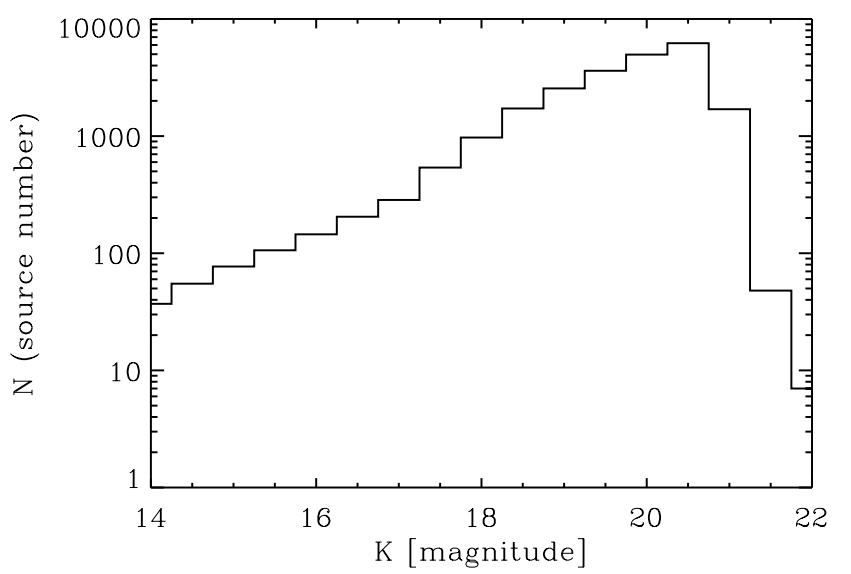

Fig. 6. Histogram of the $K$-band magnitudes in the NTT-SOFI images. Completeness in detecting objects is lost for $K>20.5$.

(FORS2) 12 optical images. The summed pre-imaging area is $\sim 530 \mathrm{arcmin}^{2}$ in the central region of the Marano Field (Fig. 2). Since the field was not contiguously covered by FORS spectroscopy, the pre-images also cover only $\sim 50 \%$ of the central area of the X-ray survey.

\subsection{Target selection for optical spectroscopy}

Our target selection for the optical spectroscopy was primarily based on FORS1/FORS $2 R$-band pre-images. WFI $R$-band and SOFI $K$-band data were used in addition to support the target selection on the pre-images in case of faint counterparts. The chosen fields represent a compromise in terms of maximum survey area and source density because of limited telescope time. First priority for spectroscopic follow up was given to candidates with a likelihood of existence $M L>10$ within a $2 \sigma_{\mathrm{X}}$ error radius. The total position error is $\sigma_{\mathrm{X}}=\sqrt{\sigma_{\text {stat. }}^{2}+\sigma_{\text {syst. }}^{2}}$. The statistical errors $\sigma_{\text {stat. }}$ of the positions were calculated as described in Sect. 2.1. The systematic errors are caused by uncertainties in the spacecraft attitude, errors in the linearisation of the detector coordinates, and undersampling of the PSF (in particular for the PN camera). At this stage, we allowed a systematic position error of $2.0^{\prime \prime}$, see Sect. 3.2 for a more accurate estimation of the systematic position errors.

The multi-object spectroscopy masks were designed with the software fims 2 provided by ESO. For all spectroscopic targets we used straight slitlets with a nominal length of ten arcseconds and width of one arcsecond. For extended sources or closely spaced candidate counterparts, a different slit length, 6-14 arcsec, was used. Still available mask positions were filled with candidate objects not fulfilling the main selection criteria and with additional random non-X-ray emitting galaxies.

\subsection{Spectroscopic observations}

The FORS1 multi-object spectroscopy (MOS) run with six masks was obtained on November 20th, 2000 by using grism GRIS_150I+17. No order separation filter was used. The exposure time for every mask was $2400 \mathrm{~s}$. The seeing was $0.7 \pm$ 0.1 arcsec.

The second run was performed with FORS2 in spectroscopic mask mode (MXU) from November 27th-30th, 2002. We aimed for an exposure time of $3 \times 1800 \mathrm{~s}$ per mask, but weather and time constraints required some deviations from this general 
Table 2. Details of FORS2 spectroscopic mask mode observation (see Fig. 2).

\begin{tabular}{cccc}
\hline \hline Mask & $\begin{array}{c}\text { Observation } \\
\text { (each 1800 s) }\end{array}$ & Filter & $\begin{array}{c}\text { Seeing } \\
\text { (in arcsec) }\end{array}$ \\
\hline mask1 & 3 & none & 0.63 \\
mask2 & 4 & none & 1.36 \\
mask3 & 3 & none & 1.05 \\
mask4 & 3 & none & 0.78 \\
mask5 & 3 & none & 0.93 \\
mask6 & 3 & GC 375 & 0.75 \\
mask7 & 3 & none & 0.80 \\
mask8 & 3 & none & 0.81 \\
mask9 & 2 & none & 0.66 \\
mask10 & 3 & none & 0.76 \\
mask11 & 1 & none & 0.96 \\
mask12 & 2 & none & 0.64 \\
\hline
\end{tabular}

Table 3. Setups for the optical spectroscopy.

\begin{tabular}{rcc}
\hline \hline & FORS1 & FORS2 \\
\hline Grism & GRIS_150I+17 & GRIS_150I+27 \\
Dispersion $[\AA / \mathrm{mm}]$ & 230 & 225 \\
Pixel size $[\mu \mathrm{m}]$ & $24 \times 24$ & $15 \times 15$ \\
Step width $[\AA$ A $/$ Pixel] & 5 & 3 \\
\hline
\end{tabular}

Table 4. Origin of optical counterparts to XMM sources.

\begin{tabular}{lr}
\hline \hline Obtained optical spectra for X-ray sources & 207 \\
\hline $\begin{array}{l}\text { Positive literature classifications by Marano } \\
\text { et al. (1988) and Zitelli et al. (1992) (partly reobserved) }\end{array}$ & 30 \\
\hline $\begin{array}{l}\text { New optical counterparts of ROSAT sources } \\
\text { Zamorani et al. (1999) (partly reobserved) }\end{array}$ & 14 \\
\hline New positive spectroscopic classifications of XMM sources & 96 \\
\hline Total positive spectroscopic classifications of XMM sources & 140 \\
\hline
\end{tabular}

scheme (Table 2). Grism GRIS 150I+27 was used for all masks. To prevent second order contamination, the first mask (mask6) in the observing sequence was observed through filter GC 375, which limited the wavelength range from 3850 to $7500 \AA$. A comparison with the second mask without an order separating filter showed that the contamination effect is negligible. Hence, for all following masks the filter was removed from the light path resulting in a wavelength range for central targets of the final spectra from 3500 to $10000 \AA$.

\subsection{Spectroscopic data reduction}

The reduction of the data was accomplished by a semiautomatic pipeline coded in MIDAS. It was specially designed to reduce FORS2-MXU data with as little interaction with the user as possible. After modifications this code was also used to reduce the FORS1-MOS data. The bias correction was done in the standard manner with careful attention to possible time dependence on the bias level and dark current. An ordinary flat field correction was used to rectify the pixel-to-pixel variation.

\subsubsection{Wavelength calibration}

We established a 2-d wavelength calibration in the pipeline. This allowed us to correct the distortion perpendicular to the dispersion direction and significantly improved the signal-to-noise ratio of the extracted spectra. The calibrated wavelength range is

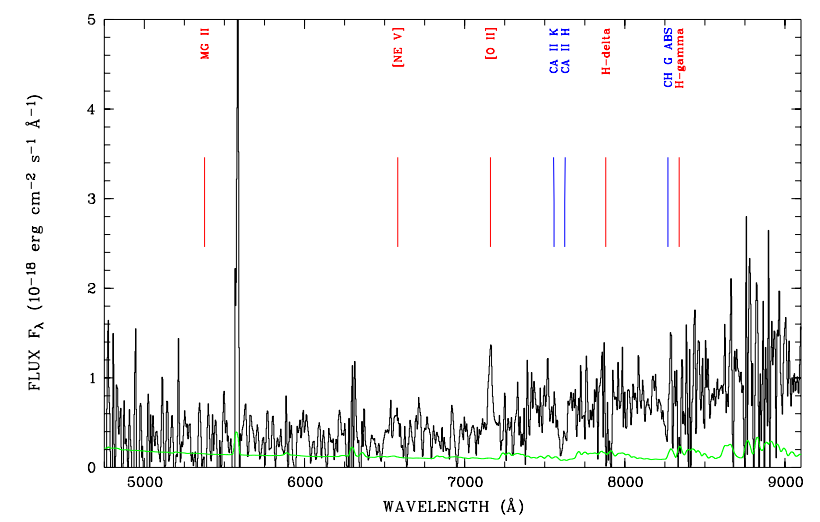

Fig. 7. Optical atmospheric absorption corrected, wavelength and flux calibrated spectrum for X-ray source 480 . The black spectrum marks the object's spectrum. The green line (see the online colour version of the manuscript) shows the error spectrum. Possible emission and absorption features in the spectrum are labelled. The spectral feature at $5580 \AA$ is spurious due to incomplete substraction of a night sky line.

3500-10000 ̊. However, many spectra were located close to the edge of the CCD. Hence, there are substantial variations in the wavelength range of the spectra. The average uncertainty of the wavelength calibration is $0.2 \AA$ but reaches a maximum of $\Delta \lambda \pm 1.5 \AA$ at long and short wavelength ends. Further details on the optical setups can be found in Table 3 . We estimate the spectral resolution finally achieved by measuring the width of the arc lines in the lamp spectra to $\Delta \lambda \sim 21 \AA$ (FWHM).

\subsubsection{Object and sky definition}

The aquisition slit-through images were used to roughly define the position and width of every single slit in an image. For an optimal definition of the object and sky region in the spectrum, the pipeline then displays intensity profiles in graphic windows and an image of the corresponding spectrum. After a careful inspection of these pipeline outputs, the object and the sky region were defined manually for every single spectrum. Consequently, bad pixel/lines/colums in the raw data can be excluded for the extraction and scientific misinterpretation of artifacts in the final spectra can be avoided. Whenever possible, two sky regions, on both sides of the target spectrum, were defined.

\subsubsection{Extraction of spectra}

The extraction is based on an optimal extraction algorithm (Horne 1986) including a cosmic ray rejection. Extensive tests determined the optimal extraction parameters. Standard flux calibration was applied. Since FORS2-mask6 was observed with an additional filter, a different standard star was used. All spectra were corrected for atmospheric absorption using the standard ESO extinction correction function scaled to the given airmass.

The $n$ individual spectra of a given counterpart object were combined to form one single final spectrum. The individual spectra were firstly normalised to the same mean intensity. The normalisation factor was determined from an analysis of all spectra in a given mask. The final spectrum is the uncertaintyweighted mean spectrum. 


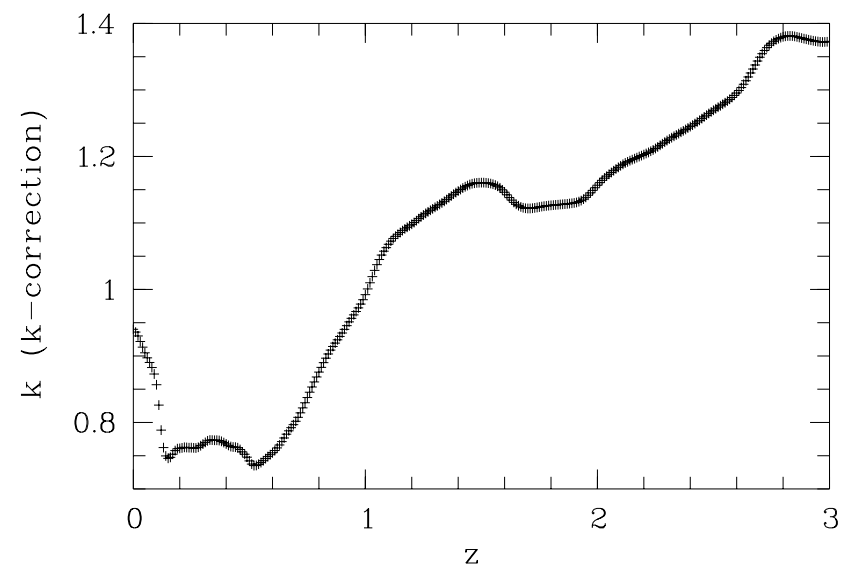

Fig. 8. Adopted mean k correction from observed $R$-band to rest-frame $M_{\mathrm{B}}$, for broad emission line AGN.

Table 5. X-ray counterpart distribution.

\begin{tabular}{lrr}
\hline \hline Object class & Total number & Percentage \\
\hline Broad emission line objects (B) & 89 & $63 \%$ \\
Narrow emission line objects (N) & 36 & $26 \%$ \\
Galaxies (G) & 6 & $4 \%$ \\
Stars (S) & 9 & $7 \%$ \\
\hline
\end{tabular}

Table 6. Properties of the core sample.

\begin{tabular}{lc}
\hline \hline X-ray sources $\left(t_{\mathrm{PN}}>6 \mathrm{ks}, M L>10\right)$ & 170 \\
Optically identified X-ray counterparts & 158 \\
Spectroscopically classified optical counterparts & 110 \\
\hline
\end{tabular}

\section{Spectroscopic classification of the X-ray counterparts}

The spectroscopic classification of the individual X-ray sources was primarily based on the FORS1 and FORS2 spectra. In addition, we used optical and X-ray images for a reliable identification (see Online Material, Appendix C). Broad emission line objects were immediately accepted as X-ray counterparts. Narrow emission line galaxies and normal galaxies were accepted as X-ray counterparts, if no other optical candidate was found within the position error range. Stars were regarded as likely counterparts if the X-ray colours indicated a soft X-ray spectrum compatible with coronal emission of $\leq 1 \mathrm{keV}$. Every $\mathrm{X}$-ray identification was confirmed by at least two individuals.

For the large majority of our spectra the signal-to-noise ratio $(S N R)$ is sufficient to give reliable classifications and redshifts. Like other X-ray identification surveys, we encounter the problem of difficult classification of the optical spectra below a certain $S N R$. For spectra with a $S N R=3-5$, the identification of narrow emission lines was still possible. However, at this $S N R$ faint broad emission lines and normal galaxy spectra are very difficult to identify. Figure 7 shows a spectrum of a narrow emission line galaxy with a $S N R=2.4$ (continuum near the O II emission line), which is close to the identification limit. Only one significant narrow emission line at $\sim 7100 \AA$ is found. The spectral shape makes it reasonable to identify this line as $\mathrm{O}$ II emission line. Even though this classification is likely, an unambiguous redshift determination and classification of the type cannot be given. Spectra with a $S N R$ less then $\sim 2.5$ are not identifiable. The reliability of the redshift determination and classification of the optical spectra is given by flags in Col. (9) Flags of Table 8.
Table 7. Fractions of absorbed sources in different X-ray luminosity bins based in Fig. 19c. Only objects with $N_{\mathrm{H}}>2 \times \Delta N_{\mathrm{H}}$ are selected.

\begin{tabular}{cccc}
\hline \hline & $43 \leq \log \left(L_{\mathrm{X}}\right)<44$ & $44 \leq \log \left(L_{\mathrm{X}}\right)<45$ & $\log \left(L_{\mathrm{X}}\right) \geq 45$ \\
\hline type I & $0 \%(0 / 19)$ & $10 \%(4 / 42)$ & $0 \%(0 / 8)$ \\
type II & $43 \%(6 / 14)$ & $58 \%(7 / 12)$ & $33 \%(1 / 3)$ \\
\hline
\end{tabular}

The complete list of X-ray classification is given in Table 8. The columns are described as follows:

(1) No

Classification of a counterpart object consists of the sequence number of the X-ray source list and a suffix (A, B) to discriminate between different candidates.

(2) RA [hh:min:s] and (3) Dec [deg:min:s]

Right ascension and declination of the optical candidate counterpart (J2000).

(4) Dist OX $_{\text {[arcsec] }}$

Spatial offset between the X-ray and optical positions.

(5) $K$

SOFI $K$-band magnitude of the spectroscopically classified candidate, whenever available.

(6) $R$

WFI $R$-band magnitude of the spectroscopically classified candidate, whenever possible.

\section{(7) Class}

Spectroscopic classification of the identified object. S-star, $\mathrm{G}$ - normal galaxy (no emission lines), $\mathrm{N}$ - narrow emission line galaxy with unresolved emission lines (at $6000 \AA$ our spectral resolution of $21 \AA$ corresponds to $1050 \mathrm{~km} \mathrm{~s}^{-1}$ ), $\mathrm{B}$ - broad emission line object (all measured line widths have $v_{\text {FWHM }}>2500 \mathrm{~km} \mathrm{~s}^{-1}$ ), and ? - undefined object.

(8) $z$

Spectroscopic redshift of the identified object. The redshift is taken from the literature for objects with " 1 -." and " 0 -." in Col. (9) Flags. Column (15) Rem. states the source of redshift determination and classification.

\section{(9) Flags}

X-ray identification flag, redshift flag, and classification reliability flag. The first number $(0,1)$ marks whether a spectroscopically classified object was accepted as X-ray counterpart. Objects which we consider to be the correct identification of the $\mathrm{X}$-ray source are flagged by " 1 ", while " 0 " flags objects not considered as the X-ray source.

The second (middle) flag states the redshift reliablility. A redshift flag " 1 " means a reliable, well-established redshift determined by several spectral features. " 0 " marks objects where the redshift determination relies on a single but reasonable spectral feature.

The third (last) flag characterises the classification reliability. A flag " 1 " marks that the object type as given in "(7) Class" is well established and reliable. Flag " 0 " indicates an uncertain classification of the object type. Either high SNR spectral features of the object do not allow a proper classification or the optical spectra do not allow to give a reliable classification of the object type because of a low $S N R$ and/or insufficient wavelength 


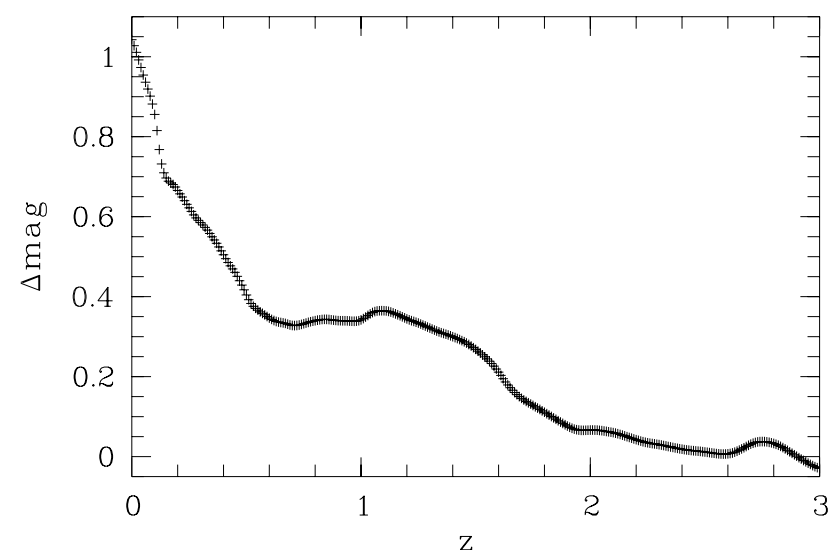

Fig. 9. Adopted conversion term to estimate $A B$ magnitudes at fixed rest-frame wavelength $\lambda=2500 \AA$ from observed $R$-band magnitudes.

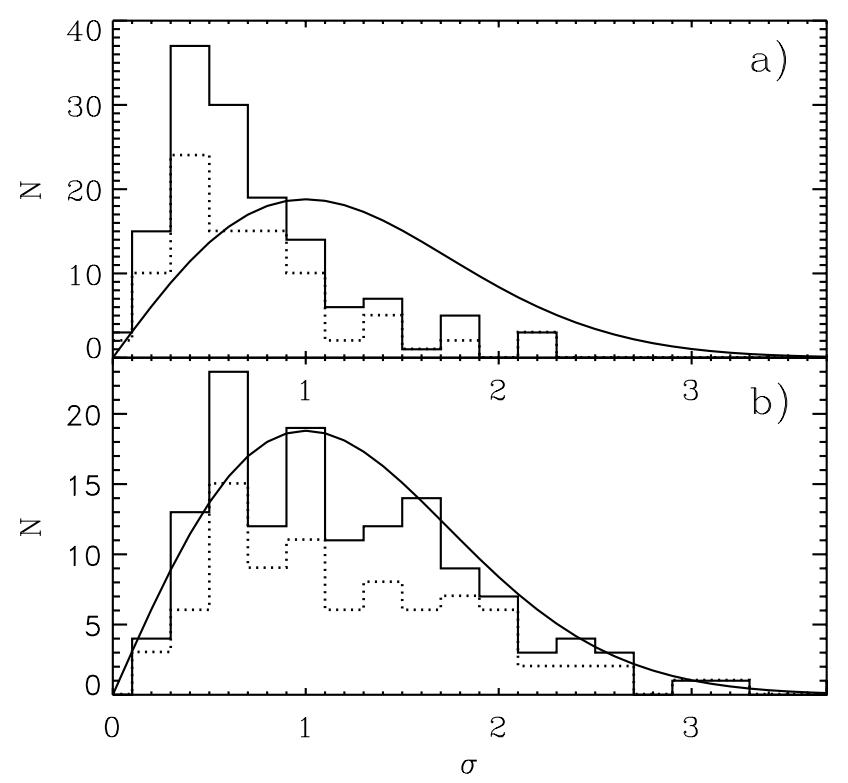

Fig. 10. a) Number of objects identified as X-ray counterparts vs. difference in X-ray and optical position scaled to a $1 \sigma_{\mathrm{X}}$ position error with a systematic X-ray position error of 2 arcsec. The smooth line represents the theoretical distribution. The distribution of type I AGN, which are a class of well-accepted X-ray emitters, is plotted as a dotted line. The solid line characterises the complete sample including type I AGN, type II AGN, galaxies, and stars. b) The $1 \sigma_{\mathrm{X}}$ position error was adjusted to the observed distribution by reducing the systematic X-ray position error from $2^{\prime \prime}$ to $0.7^{\prime \prime}$. The complete sample agrees well with the type I AGN distribution, which gives evidence of a marginally false match rate.

coverage of the optical spectra. The latter is illustrated in Fig. 7. The narrow $\mathrm{O}$ II line indicates a narrow emission line galaxy. However, the SNR of the spectrum does not allow us to judge the existence of a broad $\mathrm{Mg}$ II line and, hence, a classification as type I AGN. Since C IV and C III are outside the spectrum's wavelength range, $\mathrm{Mg}$ II is the only possible broad-line feature. The most likely classification of this object is a narrow emission line galaxy. However, the given arguments show that a classification as type I AGN cannot be excluded. Therefore, the classification flag for this object is " 0 ". Objects with " 1 - -" and " 0 - - " have only an X-ray identification flag since their redshift and classification relies on follow-up surveys previously done in the Marano Field (see Col. (15) Rem.).
(10) $\log \left(L_{X_{O B S}}\left[\operatorname{erg~s}^{-1}\right]\right)$

Observed rest-frame X-ray luminosity (logarithmic units) in the $0.2-10 \mathrm{keV}$ energy band calculated by using Eq. (1). The $k$-correction vanishes since we assume an energy index $\alpha=-1$ with $F_{v} \sim v^{\alpha} \sim v^{1-\Gamma}$ ( $\Gamma$-photon index) based on Alexander et al. (2003) and Mainieri et al. (2002). The luminosity distance $d_{\mathrm{L}}$ was computed by the analytical fit for flat cosmologies with $\Omega_{\mathrm{m}}=0.3, \Omega_{\Lambda}=0.7, H_{0}=70 \mathrm{~km} \mathrm{~s}^{-1} \mathrm{Mpc}^{-1}$ following Szokoly et al. (2004).

$L_{\mathrm{X}}=\frac{4 \pi d_{\mathrm{L}}^{2}}{(z+1)^{\alpha+1}} f_{\mathrm{X}}$.

(11) $M_{B}$

Absolute magnitudes $M_{\mathrm{B}}$ (in the Johnson system) were estimated only for type I AGN using the relation

$M_{\mathrm{B}}=R+5-5 \log \left(d_{\mathrm{L}} / \mathrm{pc}\right)+K(z)$,

where $d_{\mathrm{L}}$ is the luminosity distance and $K(z)$ is the customary $k$-correction term. In our case, this term includes the transition from observed-frame $R$-band to rest-frame $B$-band, assuming a mean spectral energy distribution for all sources, and also the $(1+z)$ bandwidth stretching factor. For the type I AGN we computed $K(z)$ from the composite SDSS quasar spectrum (Vanden Berk et al. 2001). The resulting graph is shown in Fig. 8.

(12) $\alpha_{O X}$

The broad band spectral index $\alpha_{\mathrm{OX}}$ roughly characterises the UV-X-ray spectral energy distribution by connecting the restframe points at $2500 \AA$ and $1 \mathrm{keV}$ with a simple power-law, $F_{v} \propto v^{-\alpha_{\mathrm{Ox}}}$. For each broad emission line AGN we estimated its flux at a fixed rest-frame wavelength of $\lambda=2500 \AA$, applying the relation

$m_{\mathrm{AB}}(2500 \AA)=R+\Delta m(z)$,

where $R$ is the quoted $R$-band magnitude, $m_{\mathrm{AB}}(2500 \AA)$ is the predicted magnitude at $2500 \AA$ - expressed in the AB system for easy conversion into monochromatic fluxes -, and $\Delta m(z)$ is a redshift-dependent term (similar, but not identical to the $k$-correction) that also accounts for the zeropoint transformation from the Vega to the $\mathrm{AB}$ system. Our adopted $\Delta m(z)$ relation, again computed from the SDSS composite quasar spectrum of Vanden Berk et al. (2001), is shown in Fig. 9. Notice that at the typical redshifts of $z \sim 1.5$ of our broad line AGN, the observed $R$-band approximately traces a rest-frame wavelength of $\lambda_{\text {rest }} \sim 2600 \AA$, implying that the spectral energy distribution corrections are small. The resulting $\mathrm{AB}$ magnitudes are then converted into fluxes following the definition of the AB system (Oke \& Gunn 1983):

$m_{\mathrm{AB}}(2500 \AA)=-2.5 \log \left(F_{v}(2500 \AA)\right)-48.60$,

where $F_{v}(2500 \AA)$ is given in $\mathrm{erg} \mathrm{s}^{-1} \mathrm{~cm}^{-2} \mathrm{~Hz}^{-1}$. The X-ray flux at $1 \mathrm{keV}$ is computed by:

$f(0.2-10 \mathrm{keV})=F_{v}(1 \mathrm{keV}) \int_{0.2 \mathrm{keV}}^{10 \mathrm{keV}} E^{\alpha} \mathrm{d} E \quad$ with $\alpha=-1$.

Hence, the broad band spectral index is obtained as

$\alpha_{\text {ox }}=\frac{\log \left(F_{v}(2500 \AA)\right)-\log \left(F_{v}(1 \mathrm{keV})\right)}{\log (v(1 \mathrm{keV}))-\log (v(2500 \AA))}$. 


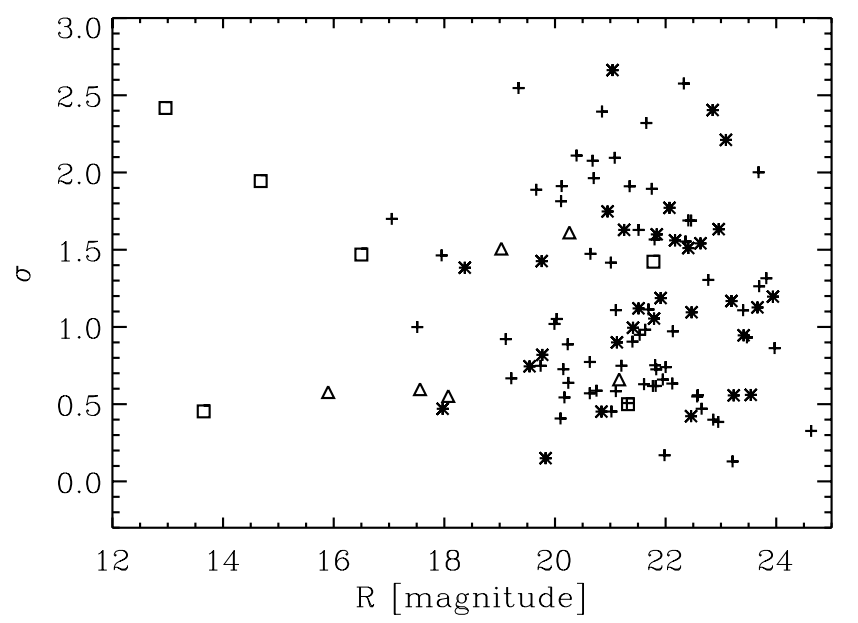

Fig. 11. WFI $R$-band magnitude vs. difference in X-ray and optical position scaled to a $1 \sigma_{\mathrm{X}}$ position error with a systematic X-ray position error of 0.7 arcsec. Crosses mark type I AGN, asterisks type II AGN, triangle galaxies, and rectangles stars.

\section{(13) $N_{H}\left[\mathrm{~cm}^{-2}\right]$}

X-ray absorbing hydrogen column density in units of $10^{22} \mathrm{~cm}^{-2}$ (see Sect. 4.4).

(14) $\log \left(L_{X_{I N T}}\left[\operatorname{erg~s}^{-1}\right]\right)$

Intrinsic rest-frame $X$-ray luminosity (logarithmic units) in the 0.2-10 keV energy band after X-ray flux correction for the absorbing hydrogen column density. Calculation uses Eq. (1).

\section{(15) Rem.}

Remarks for individual objects: 1 - optically selected and spectroscopically classified quasar by Marano et al. (1988); 2 - optically selected and spectroscopically classified quasar by Zitelli et al. (1992); 3 - ROSAT X-ray source with spectroscopic classification and redshift determination by Zamorani et al. (1999); 4 - ROSAT X-ray source with no or wrong identification by Zamorani et al. (1999); 5 - unclassified radio objects within 5.0", Gruppioni et al. (1999); 6 - spectroscopic classification and redshift taken from Teplitz et al. (2003); 7 - radio source, spectroscopic classification and redshift taken from Gruppioni et al. (1999), and C - individual comment to an object (see Sect. 3.1).

\subsection{Comments to individual objects}

9A: New redshift determined for this source, formerly known as Zamorani et al. (1999) X043-12.

15A: Object 15A is not regarded as the optical counterpart, since the identification of the line features is uncertain and the positional offset is rather large. A very faint object at the detection limit lies in the X-ray error circle. A lower limit of $f_{\mathrm{X}} / f_{\text {opt }} \sim 65$ for this source was estimated by assuming a limiting magnitude of $R=24$.

17A: Source 17 is a likely $X$-ray blend with large contrast between the two individual sources. Object 17A is a unique identification of the brighter X-ray source, whose X-ray flux is likely to be overestimated due to blending.

20A and 20B: Zamorani et al. (1999) identified source 20 with the M star 20B. Some contribution of the M star's Xray flux cannot be excluded, but the identification with the type II AGN (object 20A) seems more likely, since the X-ray colours indicate a relatively hard spectrum.

22A and 22B: $X$-ray source 22 is a probable blend. Objects 22A and 22B fall on top of the two suspected point sources. We regard both optical objects as likely counterparts of the X-ray blend and distribute the measured X-ray flux evenly between the two sources.

26A: New redshift determined for this source, formerly known as Zamorani et al. (1999) X301-29.

32A: NV strongest emission line in the spectrum (brighter than $\mathrm{Ly}-\alpha$ ). Narrow emission lines are present and the object is classified as "N" (narrow emission line galaxy). However, all narrow emission lines have underlying broad components. Therefore, the classification flag is set to " 0 ".

35A and 35B: Two galaxies at equal redshift, the brighter galaxy $35 \mathrm{~A}$ is regarded as the $\mathrm{X}$-ray counterpart. The possibility that the X-ray source is a galaxy cluster cannot completely excluded, but an X-ray extent is not obvious. For 35A a new redshift is determined (update to Zamorani et al. 1999, X022-48).

42A: New redshift determined for this source, formerly known as Zamorani et al. (1999) X031-24.

46A: X-ray blend with major contribution from the southwestern component with type I AGN 46A as counterpart.

47A and 47B: Two narrow emission line galaxies at equal redshift. The possibility that the X-ray source is a galaxy cluster cannot completely excluded, but an X-ray extent is not obvious. $47 \mathrm{~A}$ is assumed to be the optical counterpart.

63A: Unresolved narrow emission lines are present and the object is classified as "N" (narrow emission line galaxy). However, the C IV emission line has an underlying broad component. Therefore, the classification flag is set to " 0 ".

66A: The identification is not unique, since the optical image reveals a possible second object inside the $1 \sigma$-X-ray position error circle.

133A: Narrow emission lines are present. The object is classified as "N" (narrow emission line galaxy). However, Ly- $\alpha$ shows a resolved, broad base. Therefore, the classification flag is set " 0 ".

151A: Probably a spurious detection of the X-ray source. The source is kept in the source list for the formal reason of having an $M L=5.2$, but is not considered further.

191A: Optical spectrum in Zitelli et al. (1992) indicates a type I AGN with typical broad emission lines. However, this object shows the lowest X-ray-to-optical flux ratio of all AGN in our sample. $f_{\mathrm{X}} / f_{\mathrm{opt}}=0.04$ suggests an X-ray faint AGN. Since this object was not detected by ROSAT, it is unlikely that the extreme $f_{\mathrm{X}} / f_{\text {opt }}$-ratio is due to a temporary low X-ray state of the object. Furthermore, it is one of the type I objects with intrinsic absorption $\left(N_{\mathrm{H}}=\left(5.75_{-2.81}^{+3.83}\right) \times 10^{22} \mathrm{~cm}^{-2}\right)$.

217A and 217B: $X$-ray blend, the identification of 217A with the southeastern component seems unambiguous, the identification of $217 \mathrm{~B}$ with the northwestern component is not unique, since a similarly bright, close-by, but still unidentified object is present at the same distance from the X-ray source.

224A and 224B: Two galaxies at equal redshift, no obvious $\mathrm{X}$-ray extent.

253A and 253B: Two objects at similar redshift with 2 mag difference in the optical. The brighter object $253 \mathrm{~A}$ is regarded as the identification.

280A: Object is classified as " $\mathrm{B}$ " since C III is well resolved with $\Delta \lambda \sim 72 \AA$ (FWHM). Ly- $\alpha$ and C IV are narrow emision lines, but show strong absorption with broad underlying components. The classification flag is set "0". Possible broad absorption line quasar. 
Table 8. Optical properties of candidate counterparts of Marano XMM-Newton X-ray sources. For a detailed explanation of the individual columns see Sect. 3.

\begin{tabular}{|c|c|c|c|c|c|c|c|c|c|c|c|c|c|c|}
\hline (1) & (2) & (3) & (4) & (5) & (6) & (7) & (8) & (9) & (10) & (11) & (12) & (13) & (14) & (15) \\
\hline No & RA & Dec & Dist $_{\mathrm{OX}}$ & $K$ & $R$ & Class & $z$ & Flags & $\log \left(L_{\mathrm{X}_{\mathrm{OBS}}}\right)$ & $M_{\mathrm{B}}$ & $\alpha_{\mathrm{OX}}$ & $N_{\mathrm{H}}$ & $\log \left(L_{\mathrm{X}_{\mathrm{INT}}}\right)$ & Rem. \\
\hline $1 \mathrm{~A}$ & 31549.6 & -551812 & 1.52 & 17.14 & 20.39 & B & 0.808 & $1--$ & 44.70 & -22.26 & 1.32 & $0.00_{-0.00}^{+0.01}$ & 44.70 & 2,3 \\
\hline $2 \mathrm{~A}$ & 31547.5 & -552904 & 1.37 & - & 19.66 & B & 0.464 & $1--$ & 44.59 & -21.66 & 1.22 & $0.00_{-0.00}^{+0.04}$ & 44.59 & 2 \\
\hline $3 \mathrm{~A}$ & 31732.7 & -552026 & 1.35 & - & - & B & 0.406 & 1 - - & 44.24 & - & - & $0.28_{-0.12}^{+0.00}$ & 44.44 & 2 \\
\hline $4 \mathrm{~A}$ & 31334.0 & -552643 & 1.26 & - & 17.05 & B & 0.987 & 111 & 45.28 & -26.04 & 1.74 & $0.00_{-0.00}^{+0.01}$ & 45.28 & 1 \\
\hline $5 \mathrm{~A}$ & 31650.4 & -551109 & 0.78 & - & 17.51 & B & 2.531 & 1 - - & 45.63 & -27.79 & 2.00 & $0.00_{-0.00}^{+0.01}$ & 45.63 & 1,3 \\
\hline $6 \mathrm{~A}$ & 31605.8 & -551539 & 1.12 & 17.79 & 20.64 & B & 0.636 & 111 & 44.01 & -21.49 & 1.46 & $0.00_{-0.00}^{+0.01}$ & 44.01 & 1,3 \\
\hline $7 \mathrm{~A}$ & 31505.6 & -550942 & 0.13 & 18.25 & 21.98 & B & 0.501 & 111 & 43.59 & -19.56 & 1.30 & $0.00_{-0.00}^{+0.01}$ & 43.59 & 4 \\
\hline $8 \mathrm{~A}$ & 31456.0 & -552007 & 1.17 & 15.53 & 19.03 & G & 0.387 & 111 & 43.32 & - & - & $2.09_{-0.73}^{+0.00}$ & 43.90 & 3,5 \\
\hline $9 \mathrm{~A}$ & 31509.9 & -551313 & 1.18 & 18.81 & 22.63 & $\mathrm{~N}$ & 1.427 & 111 & 44.64 & - & - & $0.33_{-0.20}^{+0.18}$ & 44.73 & $4, \mathrm{C}$ \\
\hline $10 \mathrm{~A}$ & 31328.3 & -551018 & 0.49 & 16.98 & 20.24 & B & 1.378 & 111 & 45.05 & -23.58 & 1.45 & $0.23_{-0.16}^{+0.18}$ & 45.12 & 3 \\
\hline $11 \mathrm{~A}$ & 31511.3 & -550927 & 0.35 & 17.98 & 21.02 & B & 1.192 & 111 & 44.51 & -22.45 & 1.47 & $0.00_{-0.00}^{+0.01}$ & 44.51 & 2,3 \\
\hline $12 \mathrm{~A}$ & 31432.4 & -551440 & 0.46 & 8.87 & - & S & 0.000 & 1 - - & - & - & - & $0.00_{-0.00}^{+0.00}$ & - & 3 \\
\hline $13 \mathrm{~A}$ & 31350.9 & -551838 & 1.54 & 18.78 & 21.35 & B & 0.500 & $1--$ & 43.66 & -20.18 & 1.37 & $0.00_{-0.00}^{+0.01}$ & 43.66 & 3 \\
\hline $14 \mathrm{~A}$ & 31638.0 & -550637 & 1.71 & 17.47 & 20.68 & B & 0.854 & 1 - - & 44.28 & -22.09 & 1.47 & $0.00_{-0.00}^{+0.01}$ & 44.28 & 2,3 \\
\hline $15 \mathrm{~A}$ & 31551.7 & -550817 & 9.58 & - & 21.69 & $\mathrm{~N}$ & 0.331 & 000 & - & - & - & - & - & $4, \mathrm{C}$ \\
\hline $16 \mathrm{~A}$ & 31538.3 & -550140 & 0.41 & 19.40 & 21.30 & B & 1.374 & 111 & 44.88 & -22.51 & 1.33 & $0.00_{-0.00}^{+0.01}$ & 44.88 & 3 \\
\hline $17 \mathrm{~A}$ & 31528.9 & -551027 & 3.62 & 18.49 & 21.27 & B & 2.161 & 111 & 45.15 & -23.69 & 1.48 & $0.00_{-0.00}^{+0.19}$ & 45.15 & $2,3, \mathrm{C}$ \\
\hline $18 \mathrm{~A}$ & 31421.1 & -552405 & 2.02 & - & 21.65 & B & 0.614 & 1 - - & 43.69 & -20.40 & 1.41 & $0.05_{-0.05}^{+0.08}$ & 43.72 & 3 \\
\hline $19 \mathrm{~A}$ & 31525.2 & -551827 & 0.82 & 19.78 & 22.13 & B & 1.573 & 111 & 44.53 & -22.03 & 1.42 & $0.00_{-0.00}^{+0.16}$ & 44.53 & 3 \\
\hline $20 \mathrm{~A}$ & 31621.5 & -551759 & 0.63 & 17.33 & - & $\mathrm{N}$ & 2.207 & 111 & 45.01 & - & - & $0.83_{-0.44}^{+0.02}$ & 45.12 & $\mathrm{C}$ \\
\hline $20 B$ & 31621.1 & -551801 & 4.11 & 13.28 & 16.72 & $S$ & 0.000 & 011 & - & - & - & - & - & 3 \\
\hline $21 \mathrm{~A}$ & 31626.1 & -552251 & 1.38 & 12.16 & 16.50 & S & 0.000 & 1 - - & - & - & - & $0.00_{-0.00}^{+0.02}$ & - & 3 \\
\hline $22 \mathrm{~A}$ & 31519.7 & -550225 & 4.63 & 19.41 & 23.33 & B & 1.78 & 111 & 44.63 & -21.19 & 1.11 & $0.00_{-0.00}^{+0.10}$ & 44.63 & $\mathrm{C}$ \\
\hline $22 \mathrm{~B}$ & 31520.1 & -550234 & 4.62 & 17.36 & 19.98 & B & 1.353 & 111 & 44.34 & -23.79 & 1.66 & $0.00_{-0.00}^{+0.00}$ & 44.34 & $1,3, \mathrm{C}$ \\
\hline $23 \mathrm{~A}$ & 31432.1 & -551959 & 1.24 & 18.32 & 21.01 & B & 1.271 & 111 & 44.37 & -22.62 & 1.56 & $0.00_{-0.00}^{+0.02}$ & 44.37 & 1 \\
\hline $25 \mathrm{~A}$ & 31534.7 & -551926 & 0.85 & 18.12 & - & B & 1.430 & 111 & 44.48 & - & - & $0.00_{-0.00}^{+0.03}$ & 44.48 & 3 \\
\hline $26 \mathrm{~A}$ & 31436.0 & -551403 & 0.80 & 17.90 & 20.23 & B & 1.19 & 111 & 44.15 & -23.24 & 1.76 & $0.00_{-0.00}^{+0.00}$ & 44.15 & $4, \mathrm{C}$ \\
\hline $27 \mathrm{~A}$ & 31538.7 & -552219 & 1.74 & 18.04 & 21.75 & B & 1.190 & 111 & 44.23 & -21.72 & 1.46 & $0.00_{-0.00}^{+0.01}$ & 44.23 & 3 \\
\hline $28 \mathrm{~A}$ & 31507.6 & -550456 & 0.64 & 17.75 & 20.15 & B & 1.315 & 111 & 44.41 & -23.56 & 1.71 & $0.00_{-0.00}^{+0.01}$ & 44.41 & 2,3 \\
\hline $29 \mathrm{~A}$ & 31511.1 & -551530 & 0.42 & 9.28 & - & S & 0.000 & 1 - - & - & - & - & $0.00_{-0.00}^{+0.01}$ & - & 3 \\
\hline $32 \mathrm{~A}$ & 31547.0 & -551755 & 1.44 & 17.75 & 22.96 & $\mathrm{~N}$ & 2.727 & 110 & 45.09 & - & - & $14.5_{-8.2}^{+7.9}$ & 45.55 & $\mathrm{C}$ \\
\hline $33 \mathrm{~A}$ & $\begin{array}{lll}3 & 13 & 19.4\end{array}$ & -551617 & 0.62 & 17.18 & 19.21 & B & 2.166 & 1 - - & 45.05 & -25.76 & 1.88 & $0.10_{-0.10}^{+0.33}$ & 45.07 & 1 \\
\hline $34 \mathrm{~A}$ & 31559.1 & -552637 & 1.72 & 18.32 & 20.70 & B & 0.983 & 1 - - & 44.36 & -22.38 & 1.50 & $0.00_{-0.00}^{+0.01}$ & 44.36 & 3 \\
\hline $35 \mathrm{~A}$ & 31503.2 & -551906 & 0.67 & 16.17 & 19.54 & $\mathrm{~N}$ & 0.391 & 111 & 42.91 & - & - & $1.32_{-0.58}^{+0.59}$ & 43.39 & $4, \mathrm{C}$ \\
\hline $35 \mathrm{~B}$ & 31503.2 & -551909 & 3.28 & - & 19.54 & $\mathrm{~N}$ & 0.391 & 011 & - & - & - & - & - & $\mathrm{C}$ \\
\hline $36 \mathrm{~A}$ & 31411.1 & -551829 & 3.12 & 19.91 & - & - & - & - & - & - & - & - & - & 4 \\
\hline $38 \mathrm{~A}$ & 31630.0 & -551911 & 2.28 & 19.66 & 22.33 & B & 1.254 & 111 & 44.50 & -21.27 & 1.27 & $0.00_{-0.00}^{+0.01}$ & 44.50 & 2,3 \\
\hline $39 \mathrm{~A}$ & 31339.7 & -550151 & 0.95 & 19.24 & 23.41 & $\mathrm{~N}$ & 0.862 & 110 & 44.07 & - & - & $0.76_{-0.33}^{+0.63}$ & 44.32 & \\
\hline $41 \mathrm{~A}$ & 31607.9 & -551724 & 1.58 & 18.54 & 22.36 & B & 0.979 & 111 & 43.85 & -20.71 & 1.43 & $0.00_{-0.00}^{+0.05}$ & 43.85 & 4 \\
\hline $42 \mathrm{~A}$ & 31548.7 & -552246 & 0.94 & 19.37 & 21.63 & B & 1.54 & 111 & 44.48 & -22.47 & 1.51 & $0.00_{-0.00}^{+0.02}$ & 44.48 & $4, \mathrm{C}$ \\
\hline $43 \mathrm{~A}$ & 31332.5 & -551049 & 1.14 & 19.52 & 23.40 & B & 2.013 & 111 & 44.76 & -21.41 & 1.24 & $0.17_{-0.17}^{+0.32}$ & 44.80 & \\
\hline $44 \mathrm{~A}$ & 31346.8 & -550031 & 1.07 & 17.42 & 21.40 & B & 0.785 & 111 & 43.82 & -21.19 & 1.51 & $0.00_{-0.00}^{+0.117}$ & 43.82 & \\
\hline $44 B$ & 31347.4 & -550030 & 6.41 & - & 22.24 & $\mathrm{~N}$ & 0.480 & 000 & - & - & - & - & - & \\
\hline $45 \mathrm{~A}$ & 31451.8 & -551932 & 2.49 & - & 23.09 & $\mathrm{~N}$ & 0.584 & 100 & 43.08 & - & - & $0.69_{-0.27}^{+0.51}$ & 43.38 & 4 \\
\hline $46 \mathrm{~A}$ & 31607.0 & -552324 & 4.01 & 19.16 & 21.98 & B & 1.308 & 111 & 44.34 & -21.71 & 1.43 & $0.00_{-0.00}^{+0.09}$ & 44.34 & $\mathrm{C}$ \\
\hline $47 \mathrm{~A}$ & 31538.7 & -551044 & 1.34 & 19.06 & 23.94 & $\mathrm{~N}$ & 0.900 & 100 & 43.47 & - & - & $2.09_{-1.09}^{+1.54}$ & 43.89 & $\mathrm{C}$ \\
\hline 47B & 31539.1 & -551042 & 3.72 & 19.99 & 22.76 & $\mathrm{~N}$ & 0.89 & 000 & - & - & - & - & - & $\mathrm{C}$ \\
\hline $49 \mathrm{~A}$ & 31638.3 & -552014 & 1.66 & 16.01 & 20.26 & G & 0.454 & 111 & 43.22 & - & - & $1.45_{-0.63}^{+0.65}$ & 43.70 & \\
\hline $50 \mathrm{~A}$ & 31409.9 & -551746 & 1.25 & 18.35 & 23.66 & $\mathrm{~N}$ & 0.986 & 110 & 43.79 & - & - & $3.63_{-1.58}^{+1.63}$ & 44.30 & \\
\hline $51 \mathrm{~A}$ & 31630.6 & -551503 & 1.83 & 16.57 & 20.95 & $\mathrm{~N}$ & 0.58 & 110 & 43.37 & - & - & $0.05_{-0.05}^{+0.08}$ & 43.41 & \\
\hline $52 \mathrm{~A}$ & 31451.4 & -545714 & 1.82 & - & - & B & 1.829 & 1 - - & 44.77 & - & - & $0.00_{-0.00}^{+0.04}$ & 44.77 & 2 \\
\hline $53 \mathrm{~A}$ & 31358.8 & -551754 & 0.67 & 18.82 & 23.23 & $\mathrm{~N}$ & 1.340 & 100 & 44.07 & - & - & $3.98_{-2.93}^{+2.51}$ & 44.50 & \\
\hline $54 \mathrm{~A}$ & 31612.4 & -545910 & 2.49 & - & - & B & 2.744 & $1--$ & 45.05 & - & - & $0.00_{-0.00}^{+0.43}$ & 45.05 & 1 \\
\hline $56 \mathrm{~A}$ & 31250.9 & -551726 & 1.94 & 16.65 & 20.11 & B & 0.641 & 111 & 43.90 & -22.04 & 1.60 & $0.01_{-0.01}^{+0.08}$ & 43.91 & 1 \\
\hline $57 \mathrm{~A}$ & 31344.0 & -551924 & 2.33 & 17.11 & 20.12 & B & 0.281 & $1--$ & 42.52 & -19.93 & 1.79 & $0.01_{-0.01}^{+0.08}$ & 42.54 & 3 \\
\hline $58 \mathrm{~A}$ & 31301.7 & -552223 & 0.70 & - & 21.10 & $\mathrm{~B}$ & 0.589 & 111 & 43.69 & -20.85 & 1.49 & $0.00_{-0.00}^{+0.02}$ & 43.69 & \\
\hline
\end{tabular}


Table 8. continued.

\begin{tabular}{|c|c|c|c|c|c|c|c|c|c|c|c|c|c|c|}
\hline (1) & (2) & (3) & (4) & (5) & (6) & (7) & (8) & (9) & (10) & (11) & (12) & (13) & (14) & $(15)$ \\
\hline No & RA & Dec & Dist $_{\mathrm{OX}}$ & $K$ & $R$ & Class & $z$ & Flags & $\log \left(L_{\mathrm{X}_{\mathrm{OBS}}}\right)$ & $M_{\mathrm{B}}$ & $\alpha_{\mathrm{OX}}$ & $N_{\mathrm{H}}$ & $\log \left(L_{\mathrm{X}_{\mathrm{INT}}}\right)$ & Rem. \\
\hline $59 \mathrm{~A}$ & $\begin{array}{lll}3 & 13 & 39.4\end{array}$ & -551426 & 0.77 & 17.37 & 21.83 & $\bar{B}$ & 0.978 & 111 & 43.94 & -21.23 & 1.49 & $0.00_{-0.00}^{+0.02}$ & 43.94 & \\
\hline $60 \mathrm{~A}$ & 31426.3 & -551747 & 0.71 & 17.62 & 21.77 & B & 0.957 & 111 & 43.69 & -21.25 & 1.59 & $0.03_{-0.03}^{+0.00}$ & 43.71 & 3,5 \\
\hline $63 \mathrm{~A}$ & 31516.9 & -550602 & 1.25 & 20.37 & 23.19 & $\mathrm{~N}$ & 2.800 & 110 & 44.88 & - & - & $4.37_{-2.10}^{+11.2}$ & 45.13 & $\mathrm{C}$ \\
\hline $64 \mathrm{~A}$ & 31333.7 & -551021 & 2.32 & 17.21 & 21.51 & B & 1.165 & 111 & 43.95 & -21.91 & 1.61 & $0.00_{-0.00}^{+0.10}$ & 43.95 & \\
\hline $66 \mathrm{~A}$ & 31500.7 & -550718 & 1.23 & 18.40 & 21.41 & $\mathrm{~N}$ & 0.981 & 100 & 43.51 & - & - & $0.28_{-0.20}^{+0.00}$ & 43.63 & $\mathrm{C}$ \\
\hline $67 \mathrm{~A}$ & 31310.6 & -551313 & 1.22 & 18.27 & 21.69 & B & 0.866 & 111 & 43.85 & -21.11 & 1.49 & $0.00_{-0.00}^{+0.03}$ & 43.85 & \\
\hline $68 \mathrm{~A}$ & 31337.9 & -552303 & 1.77 & 18.28 & 21.80 & B & 0.839 & 111 & 43.82 & -20.93 & 1.47 & $0.00_{-0.00}^{+0.08}$ & 43.82 & 5 \\
\hline $69 \mathrm{~A}$ & 31415.3 & -551910 & 0.50 & 19.37 & 24.63 & B & 2.553 & 111 & 44.42 & -20.69 & 1.29 & $1.20_{-0.77}^{+1.21}$ & 44.53 & \\
\hline $70 \mathrm{~A}$ & 31621.0 & -550649 & 1.98 & 19.82 & 23.37 & - & - & - & - & - & - & - & - & \\
\hline $70 \mathrm{~B}$ & 31621.0 & -550645 & 3.35 & 18.96 & 22.15 & $\mathrm{~N}$ & 0.844 & 010 & - & - & - & - & - & \\
\hline $73 \mathrm{~A}$ & 31321.2 & -552047 & 0.51 & 18.31 & 20.10 & B & 2.706 & 111 & 45.10 & -25.31 & 1.81 & $0.00_{-0.00}^{+0.12}$ & 45.10 & 1 \\
\hline $75 \mathrm{~A}$ & 31601.2 & -550516 & 0.50 & 18.96 & 22.86 & B & 2.460 & 111 & 44.64 & -22.38 & 1.49 & $0.00_{-0.00}^{+0.034}$ & 44.64 & \\
\hline $76 \mathrm{~A}$ & 31549.7 & -550908 & 0.16 & 19.06 & 23.21 & B & 1.065 & 111 & 43.69 & -20.01 & 1.39 & $0.01_{-0.01}^{+0.00}$ & 43.69 & 3 \\
\hline $78 \mathrm{~A}$ & 31502.0 & -552611 & 1.13 & 19.17 & 23.68 & - & - & - & - & - & - & - & - & \\
\hline $80 \mathrm{~A}$ & 31359.4 & -545717 & 3.16 & - & - & B & 1.620 & $1--$ & 44.51 & - & - & $0.00_{-0.00}^{+0.05}$ & 44.51 & 1 \\
\hline $81 \mathrm{~A}$ & 31344.2 & -550152 & 4.16 & 18.86 & - & - & - & - & - & - & - & - & & \\
\hline $83 \mathrm{~A}$ & 31447.9 & -552231 & 2.33 & 19.46 & 23.68 & B & 1.650 & 111 & 44.27 & -20.63 & 1.29 & $0.00_{-0.00}^{+0.07}$ & 44.27 & \\
\hline $83 \mathrm{~B}$ & 31448.8 & -552230 & 5.51 & 18.40 & 20.70 & $\mathrm{~N}$ & 0.568 & 010 & - & - & - & - & - & 3 \\
\hline $84 \mathrm{~A}$ & 31621.6 & -552038 & 1.32 & 18.63 & 23.47 & B & 2.094 & 111 & 44.48 & -21.43 & 1.37 & $1.32_{-0.75}^{+1.27}$ & 44.64 & \\
\hline $84 \mathrm{~B}$ & 31621.9 & -552041 & 3.72 & 18.73 & 23.42 & S & 0.000 & 011 & - & - & - & - & - & \\
\hline $88 \mathrm{~A}$ & 31316.6 & -550310 & 3.31 & - & 20.85 & B & 0.739 & 111 & 43.62 & -21.62 & 1.67 & $0.00_{-0.00}^{+0.03}$ & 43.62 & \\
\hline $92 \mathrm{~A}$ & 31200.3 & -550222 & 1.85 & - & - & B & 2.425 & $1--$ & 45.22 & - & - & $0.58_{-0.58}^{+0.00}$ & 45.29 & 1 \\
\hline $100 \mathrm{~A}$ & 31243.8 & -551058 & 2.43 & - & - & B & 1.165 & 111 & 44.23 & - & - & $0.01_{-0.01}^{+0.17}$ & 44.24 & \\
\hline $104 \mathrm{~A}$ & 31647.0 & -551033 & 3.80 & - & 23.86 & - & - & - & - & - & - & - & - & \\
\hline $111 \mathrm{~A}$ & 31505.2 & -552948 & 2.42 & - & - & - & - & - & - & - & - & - & - & \\
\hline $116 \mathrm{~A}$ & 31620.7 & -551652 & 1.30 & 16.99 & - & $\mathrm{N}$ & 0.581 & 111 & 43.08 & - & - & $3.63_{-1.42}^{+1.37}$ & 43.72 & \\
\hline $120 \mathrm{~A}$ & 31313.5 & -550159 & 0.87 & - & 15.90 & G & 0.094 & $1--$ & 41.46 & - & - & $0.00_{-0.00}^{+0.42}$ & 41.46 & \\
\hline $132 \mathrm{~A}$ & 31614.3 & -551729 & 1.53 & 17.65 & 21.79 & $\mathrm{~N}$ & 1.144 & 100 & 43.70 & - & - & $0.00_{-0.00}^{+0.18}$ & 43.70 & \\
\hline $133 \mathrm{~A}$ & 31426.2 & -552113 & 1.02 & - & 23.54 & $\mathrm{~N}$ & 2.321 & 110 & 44.25 & - & - & $0.00_{-0.00}^{+0.20}$ & 44.25 & $\mathrm{C}$ \\
\hline $135 \mathrm{~A}$ & 31314.7 & -552619 & 5.41 & - & 21.95 & B & 2.033 & 111 & 44.79 & -22.88 & 1.48 & $0.00_{-0.00}^{+0.10}$ & 44.79 & \\
\hline $140 \mathrm{~A}$ & 31433.0 & -552518 & 0.91 & - & 21.84 & - & - & - & - & - & - & - & - & \\
\hline $145 \mathrm{~A}$ & 31537.1 & -551714 & 1.40 & 16.39 & 19.77 & $\mathrm{~N}$ & 0.497 & 111 & 42.31 & - & - & $100_{31}$ & 44.11 & \\
\hline $150 \mathrm{~A}$ & 31429.5 & -550605 & 0.32 & 18.15 & 23.21 & - & - & - & - & - & - & - & - & \\
\hline $151 \mathrm{~A}$ & 31650.5 & -551101 & 4.45 & - & - & - & - & - & - & - & - & - & - & $\mathrm{C}$ \\
\hline $157 \mathrm{~A}$ & 31628.0 & -550538 & 3.46 & 16.41 & 19.76 & $\mathrm{~N}$ & 0.625 & 111 & 42.79 & - & - & - & - & \\
\hline $165 \mathrm{~A}$ & 31532.2 & -551654 & 5.06 & 10.94 & 12.90 & S & 0.000 & 011 & - & - & - & - & - & \\
\hline $166 \mathrm{~A}$ & 31455.3 & -551815 & 6.85 & 17.91 & 21.45 & S & 0.000 & 011 & - & - & - & - & - & \\
\hline $171 \mathrm{~A}$ & 31351.4 & -550256 & 2.18 & 18.59 & 22.17 & $\mathrm{~N}$ & 0.800 & 111 & 43.45 & - & - & $6.31_{-2.47}^{+2.38}$ & 44.14 & \\
\hline $172 \mathrm{~A}$ & 31624.3 & -551908 & 3.17 & - & - & - & - & - & - & - & - & - & - & \\
\hline $185 \mathrm{~A}$ & 31650.8 & -552224 & 1.60 & - & - & B & 2.768 & 1 - - & 44.90 & - & - & $6.31_{-4.03}^{+7.80}$ & 45.21 & 2 \\
\hline $189 \mathrm{~A}$ & 31325.4 & -550118 & 8.94 & 19.34 & 20.47 & $\mathrm{~N}$ & 0.079 & 011 & - & - & - & - & - & \\
\hline $191 \mathrm{~A}$ & 31438.5 & -552006 & 2.27 & 15.98 & 17.95 & B & 1.045 & $1--$ & 43.44 & -25.24 & 2.40 & $5.75_{-2.81}^{+3.83}$ & 44.01 & $1, \mathrm{C}$ \\
\hline 197A & 31427.9 & -551934 & 2.27 & 17.94 & - & - & - & - & - & - & - & - & - & \\
\hline $204 \mathrm{~A}$ & 31424.6 & -550203 & 1.31 & 18.14 & 21.16 & G & 0.859 & 110 & 43.30 & - & - & $0.03_{-0.03}^{+2.26}$ & 43.32 & \\
\hline $205 \mathrm{~A}$ & 31318.9 & -552512 & 1.73 & - & - & $\mathrm{S}$ & 0.000 & 111 & - & - & - & $0.00_{-0.00}^{+0.01}$ & - & \\
\hline 205B & 31318.8 & -552507 & 4.20 & - & - & S & 0.000 & 011 & - & - & - & - & - & \\
\hline $209 \mathrm{~A}$ & 31602.2 & -550248 & 0.88 & 19.31 & 20.63 & B & 2.735 & 111 & 44.67 & -24.79 & 1.90 & $1.58_{-1.17}^{+2.50}$ & 44.80 & 2 \\
\hline $217 \mathrm{~A}$ & 31419.6 & -551643 & 3.81 & 19.35 & 22.07 & $\mathrm{~N}$ & 0.816 & 110 & 42.96 & - & - & $3.63_{-1.77}^{+2.41}$ & 43.51 & $\mathrm{C}$ \\
\hline 217B & 31419.2 & -551637 & 3.66 & 18.00 & 21.32 & $\mathrm{~N}$ & 0.645 & 010 & - & - & - & - & - & $\mathrm{C}$ \\
\hline $220 \mathrm{~A}$ & 31550.4 & -550145 & 1.75 & 18.48 & 21.53 & B & 1.529 & 111 & 44.11 & -22.55 & 1.69 & $0.00_{-0.00}^{+0.10}$ & 44.11 & \\
\hline $222 \mathrm{~A}$ & 31643.2 & -552006 & 8.05 & - & - & G & 0.82 & 000 & - & - & - & - & - & \\
\hline $223 \mathrm{~A}$ & 31647.6 & -551411 & 1.37 & - & - & $\mathrm{N}$ & 1.304 & 100 & 44.07 & - & - & $0.28_{-0.25}^{+0.45}$ & 44.16 & \\
\hline $224 \mathrm{~A}$ & 31304.9 & -551607 & 1.89 & 18.36 & 21.46 & G & 0.688 & 001 & - & - & - & -0.25 & - & $\mathrm{C}$ \\
\hline 224B & 31304.8 & -551604 & 1.70 & 17.48 & 21.51 & $\mathrm{~N}$ & 0.690 & 111 & 43.48 & - & - & $17.4_{-5.1}^{+7.9}$ & 44.48 & $\mathrm{C}$ \\
\hline $225 \mathrm{~A}$ & 31331.7 & -550046 & 3.12 & 15.89 & - & G & 0.420 & 011 & - & - & - & - & - & \\
\hline $225 \mathrm{~B}$ & 31332.2 & -550045 & 1.32 & 16.06 & 17.99 & $S$ & 0.000 & 011 & - & - & - & - & - & \\
\hline $229 \mathrm{~A}$ & 31606.5 & -551444 & 4.07 & - & 22.23 & $\mathrm{~N}$ & 0.98 & 001 & - & - & - & - & - & \\
\hline
\end{tabular}


Table 8. continued.

\begin{tabular}{|c|c|c|c|c|c|c|c|c|c|c|c|c|c|c|}
\hline (1) & (2) & (3) & (4) & (5) & (6) & (7) & (8) & (9) & (10) & (11) & (12) & (13) & (14) & (15) \\
\hline No & RA & Dec & Dist $_{\mathrm{OX}}$ & $K$ & $R$ & Class & $z$ & Flags & $\log \left(L_{\mathrm{X}_{\mathrm{OBS}}}\right)$ & $M_{\mathrm{B}}$ & $\alpha_{\mathrm{OX}}$ & $N_{\mathrm{H}}$ & $\log \left(L_{\mathrm{X}_{\mathrm{INT}}}\right)$ & Rem. \\
\hline $232 \mathrm{~A}$ & 31441.7 & -550817 & 1.74 & 19.48 & - & $\bar{B}$ & 2.520 & 111 & 44.58 & - & - & $1.20_{-0.95}^{+16.4}$ & 44.71 & \\
\hline $241 \mathrm{~A}$ & 31321.9 & -551351 & 0.95 & 15.81 & 17.56 & $\mathrm{G}$ & 0.165 & 111 & 41.85 & - & - & $\begin{array}{r}-01_{-0.01}^{+0.93} \\
0.06\end{array}$ & 41.87 & \\
\hline $242 \mathrm{~A}$ & 31604.2 & -550716 & 0.81 & 18.34 & 22.57 & B & 1.147 & 111 & 43.69 & -20.81 & 1.54 & $0.40_{-0.25}^{+0.01}$ & 43.82 & \\
\hline $246 \mathrm{~A}$ & 31356.6 & -550107 & 2.96 & 18.50 & 21.91 & $\mathrm{~N}$ & 0.944 & 110 & 43.51 & - & - & $0.52_{-0.35}^{+1.77}$ & 43.70 & \\
\hline $246 B$ & 31357.1 & -550113 & 4.75 & 20.25 & 22.64 & $\mathrm{~N}$ & 0.688 & 0 - - & - & - & - & - & - & 6 \\
\hline $253 \mathrm{~A}$ & 31438.0 & -550650 & 2.64 & 18.21 & 21.84 & $\mathrm{~N}$ & 0.517 & 110 & 42.64 & - & - & $25.1_{-15.2}^{+28.5}$ & 43.83 & $\mathrm{C}$ \\
\hline $253 B$ & 31438.1 & -550645 & 3.12 & - & 23.86 & $\mathrm{~N}$ & 0.518 & 010 & - & - & - & - & - & $\mathrm{C}$ \\
\hline $255 \mathrm{~A}$ & 31446.2 & -550952 & 4.28 & 14.66 & 17.51 & S & 0.00 & 011 & - & - & - & - & - & \\
\hline $265 \mathrm{~A}$ & 31336.7 & -550018 & 0.94 & 18.89 & 22.65 & B & 1.280 & 111 & 43.74 & -21.00 & 1.56 & $0.91_{-0.90}^{+3.29}$ & 43.94 & \\
\hline $265 B$ & 31337.0 & -550020 & 4.04 & 19.84 & 21.81 & S & 0.000 & 011 & - & - & - & - & - & \\
\hline $267 \mathrm{~A}$ & 31422.6 & -551709 & 2.71 & 18.53 & 22.46 & B & 1.495 & 111 & 43.83 & -21.56 & 1.64 & $8.32_{-4.06}^{+6.68}$ & 44.37 & \\
\hline $268 \mathrm{~A}$ & 31623.8 & -551520 & 1.17 & 18.06 & 21.20 & B & 1.22 & 111 & 43.72 & -22.34 & 1.79 & $0.00_{-0.00}^{+0.00}$ & 43.72 & 4 \\
\hline $268 \mathrm{~B}$ & 31623.9 & -551521 & 1.68 & 17.82 & 21.20 & $\mathrm{~N}$ & 0.983 & 010 & - & - & - & - & - & \\
\hline $280 \mathrm{~A}$ & 31324.8 & -551119 & 0.66 & 18.93 & 22.95 & B & 1.936 & 110 & 44.23 & -21.78 & 1.53 & $27.5_{-12.6}^{+21.1}$ & 44.97 & $\mathrm{C}$ \\
\hline $281 \mathrm{~A}$ & 31557.7 & -550602 & 1.92 & - & - & - & - & - & - & - & - & - & - & \\
\hline $300 \mathrm{~A}$ & 31501.8 & -551408 & 4.93 & 10.21 & 12.96 & S & 0.000 & 111 & - & - & - & $0.00_{-0.00}^{+0.01}$ & - & \\
\hline $308 \mathrm{~A}$ & 31317.8 & -551552 & 1.71 & - & 22.13 & - & - & - & - & - & - & - & - & \\
\hline $310 \mathrm{~A}$ & 31457.9 & -551323 & 3.21 & 18.51 & 22.41 & B & 1.01 & 111 & 43.07 & -20.72 & 1.78 & $0.05_{-0.05}^{+0.20}$ & 43.09 & \\
\hline $311 \mathrm{~A}$ & 31629.8 & -551102 & 3.88 & 18.61 & - & - & - & - & - & - & - & - & - & \\
\hline $334 \mathrm{~A}$ & 31631.6 & -551227 & 0.70 & 19.32 & 20.17 & B & 2.536 & 1 - - & 44.73 & -25.13 & 1.93 & $0.52_{-0.52}^{+0.69}$ & 44.79 & 2,3 \\
\hline $335 \mathrm{~A}$ & 31357.3 & -550432 & 0.91 & 15.96 & 17.97 & $\mathrm{~N}$ & 0.257 & 111 & 41.93 & - & - & $1.91_{-0.96}^{+1.77}$ & 42.54 & \\
\hline $345 \mathrm{~A}$ & 31316.6 & -552238 & 0.84 & - & 21.61 & B & 2.166 & 111 & 44.88 & -23.36 & 1.54 & $0.00_{-0.00}^{+0.10}$ & 44.88 & \\
\hline $360 \mathrm{~A}$ & 31311.7 & -551718 & 2.36 & 20.02 & - & B & 2.166 & 111 & 44.49 & - & - & $0.14_{-0.14}^{+0.90}$ & 44.51 & \\
\hline $361 \mathrm{~A}$ & 31314.6 & -550318 & 2.03 & - & 20.03 & B & 1.648 & 111 & 44.20 & -24.28 & 1.96 & $12.0_{-6.4}^{+9.14}$ & 44.80 & \\
\hline $364 \mathrm{~A}$ & 31412.0 & -552556 & 1.82 & - & 21.10 & B & 1.821 & 1 - - & 44.39 & -23.48 & 1.75 & $0.23_{-0.23}^{+0.36}$ & 44.45 & 2,3 \\
\hline $367 \mathrm{~A}$ & 31634.8 & -550349 & 3.92 & - & 21.08 & B & 0.981 & $1--$ & 43.97 & -21.99 & 1.61 & $2.29_{-1.46}^{+1.93}$ & 44.37 & 2 \\
\hline $371 \mathrm{~A}$ & 31620.5 & -550333 & 3.16 & - & 21.25 & $\mathrm{~N}$ & 0.802 & 110 & 43.23 & - & - & $33.1_{-10.6}^{+20.50}$ & 44.37 & \\
\hline $373 \mathrm{~A}$ & 31502.3 & -550507 & 2.53 & - & 23.69 & B & 0.953 & 101 & 43.24 & -19.33 & 1.45 & $0.00_{-0.00}^{+0.06}$ & 43.24 & \\
\hline $373 B$ & 31502.5 & -550502 & 6.11 & - & 21.87 & $\mathrm{~N}$ & 0.573 & 010 & - & - & - & - & - & \\
\hline $377 \mathrm{~A}$ & 31653.4 & -550838 & 0.81 & - & 22.91 & - & - & - & - & - & - & - & - & \\
\hline $381 \mathrm{~A}$ & 31644.3 & -552708 & 2.93 & - & 19.34 & $\mathrm{~B}$ & 1.879 & 1 - - & 45.01 & -25.32 & 1.80 & $0.00_{-0.00}^{+0.07}$ & 45.01 & 1 \\
\hline $382 \mathrm{~A}$ & 31247.3 & -551651 & 1.46 & 19.83 & 20.63 & B & 1.904 & 111 & 44.57 & -24.07 & 1.78 & $0.00_{-0.00}^{+0.00}$ & 44.57 & $\mathrm{C}$ \\
\hline $388 \mathrm{~A}$ & 31401.3 & -545956 & 0.72 & 17.27 & 19.99 & B & 0.841 & 1 - - & 44.74 & -22.75 & 1.39 & $0.00_{-0.00}^{+0.01}$ & 44.74 & 1 \\
\hline $408 \mathrm{~A}$ & 31554.6 & -551004 & 4.12 & 19.92 & 23.40 & - & - & - & - & - & - & - & - & \\
\hline $410 \mathrm{~A}$ & 31502.4 & -552745 & 4.21 & 11.01 & 14.68 & S & 0.000 & 111 & - & - & - & $0.00_{-0.00}^{+0.01}$ & - & \\
\hline $422 \mathrm{~A}$ & 31531.6 & -551045 & 4.79 & 17.22 & 21.04 & $\mathrm{~N}$ & 0.476 & 111 & 42.50 & - & - & $3.98_{-1.61}^{+2.46}$ & 43.21 & \\
\hline $437 \mathrm{~A}$ & 31533.7 & -550258 & 2.33 & 20.22 & 21.78 & $\mathrm{~S}$ & 0.00 & 111 & - & - & - & $0.01_{-0.01}^{+0.01}$ & - & \\
\hline $449 \mathrm{~A}$ & 31543.8 & -550742 & 4.28 & 18.62 & - & B & 1.204 & 111 & 43.47 & - & - & $7.59_{-4.04}^{+6.10}$ & 44.08 & 3 \\
\hline $462 \mathrm{~A}$ & 31506.0 & -551624 & 1.99 & 18.75 & - & - & - & - & - & - & - & - & - & \\
\hline $463 \mathrm{~A}$ & 31625.3 & -550839 & 0.55 & 19.07 & - & $\mathrm{N}$ & 2.531 & 111 & 44.41 & - & - & $3.63_{-2.20}^{+4.27}$ & 44.66 & \\
\hline $473 \mathrm{~A}$ & 31532.2 & -551122 & 6.40 & - & 21.64 & $S$ & 0.000 & 011 & - & - & - & $0.01_{-0.01}^{+0.20}$ & - & \\
\hline $473 B$ & 31532.9 & -551127 & 11.67 & - & 17.22 & G & 0.154 & 0 - - & - & - & - & - & - & 7 \\
\hline $475 \mathrm{~A}$ & 31352.4 & -550346 & 1.77 & 20.20 & 23.97 & B & 1.729 & 111 & 44.07 & -20.48 & 1.36 & $0.10_{-0.10}^{+0.43}$ & 44.10 & \\
\hline $480 \mathrm{~A}$ & 31653.2 & -551232 & 2.52 & - & 22.47 & $\mathrm{~N}$ & 0.921 & 100 & 43.52 & - & - & $\begin{array}{r}4.79_{-2.34}^{+3.85} \\
\end{array}$ & 44.12 & \\
\hline $485 \mathrm{~A}$ & 31527.1 & -551614 & 4.69 & 17.75 & 22.85 & $\mathrm{~N}$ & 1.263 & 100 & 43.33 & - & - & $27.5_{-15 .}^{+72.5}$ & 44.25 & \\
\hline $496 \mathrm{~A}$ & 31245.4 & -551648 & 1.38 & - & 19.74 & B & 1.906 & 111 & 44.63 & -24.96 & 1.91 & $0.00_{-0.00}^{+0.11}$ & 44.63 & $\mathrm{C}$ \\
\hline $498 \mathrm{~A}$ & 31506.1 & -551400 & 1.09 & 11.83 & 13.65 & $S$ & 0.00 & 111 & - & - & - & $0.01_{-0.01}^{+0.06}$ & - & \\
\hline $511 \mathrm{~A}$ & 31528.0 & -551314 & 0.92 & 19.88 & 22.58 & B & 2.623 & 111 & 44.22 & -22.79 & 1.75 & $0.00_{-0.00}^{+0.010}$ & 44.22 & \\
\hline $512 \mathrm{~A}$ & 31348.2 & -551309 & 1.12 & 17.90 & - & $\mathrm{N}$ & 0.465 & 110 & 42.83 & - & - & $\begin{array}{r}-91_{-1.02}^{+1.53} \\
\end{array}$ & 43.36 & $\mathrm{C}$ \\
\hline $512 \mathrm{~B}$ & 31348.3 & -551305 & 3.57 & 15.92 & - & $\mathrm{N}$ & 0.584 & $0--$ & - & - & - & - & - & $3, \mathrm{C}$ \\
\hline $518 \mathrm{~A}$ & 31457.0 & -552925 & 2.01 & - & 21.53 & - & - & - & - & - & - & - & - & \\
\hline $524 \mathrm{~A}$ & 31314.1 & -550416 & 3.54 & - & 20.46 & S & 0.000 & 011 & - & - & - & - & - & \\
\hline $531 \mathrm{~A}$ & 31338.2 & -550351 & 1.12 & 18.31 & - & B & 0.926 & 111 & 44.15 & - & - & $0.00_{-0.00}^{+0.01}$ & 44.15 & \\
\hline $535 \mathrm{~A}$ & 31653.5 & -551152 & 1.51 & - & 21.81 & B & 0.974 & 101 & 43.43 & -21.25 & 1.71 & $0.44_{-0.31}^{+0.58}$ & 43.60 & \\
\hline $536 \mathrm{~A}$ & 31450.7 & -550410 & 3.47 & - & 22.41 & $\mathrm{~N}$ & 0.333 & 111 & 41.92 & - & - & - & - & \\
\hline $543 \mathrm{~A}$ & 31250.3 & -551100 & 0.56 & 19.85 & - & B & 2.511 & 111 & 44.44 & - & - & - & - & \\
\hline $551 \mathrm{~A}$ & 31243.3 & -551201 & 3.16 & - & - & S & 0.000 & 011 & - & - & - & - & - & \\
\hline
\end{tabular}


Table 8. continued.

\begin{tabular}{|c|c|c|c|c|c|c|c|c|c|c|c|c|c|c|}
\hline (1) & (2) & (3) & (4) & $(5)$ & (6) & (7) & $(8)$ & (9) & (10) & (11) & (12) & (13) & (14) & (15) \\
\hline No & RA & Dec & Dist $_{\mathrm{OX}}$ & $K$ & $R$ & Class & $z$ & Flags & $\log \left(L_{\mathrm{X}_{\mathrm{OBS}}}\right)$ & $M_{\mathrm{B}}$ & $\alpha_{\mathrm{OX}}$ & $N_{\mathrm{H}}$ & $\log \left(L_{\mathrm{X}_{\mathrm{INT}}}\right)$ & Rem. \\
\hline 551B & 31243.0 & -551158 & 1.20 & - & - & $\bar{B}$ & 0.789 & 111 & 43.48 & - & - & $0.00_{-0.00}^{+0.20}$ & 43.48 & \\
\hline 557A & 31441.4 & -552137 & 1.27 & 15.42 & 18.07 & G & 0.16 & 111 & 41.26 & - & - & $0.00_{-0.00}^{+0.00}$ & 41.26 & \\
\hline $559 \mathrm{~A}$ & 31617.3 & -551429 & 0.99 & 17.95 & 21.95 & B & 1.180 & $1-$ & 43.82 & -21.51 & 1.60 & $\begin{array}{r}-0.00 \\
0.00_{-0.00}^{+0.02}\end{array}$ & 43.82 & 3 \\
\hline $579 \mathrm{~A}$ & 31547.3 & -551419 & 0.88 & 18.01 & 20.84 & $\mathrm{~N}$ & 0.497 & 110 & 42.43 & - & - & $0.23_{-0.12}^{+0.18}$ & 42.60 & \\
\hline $582 \mathrm{~A}$ & 31630.5 & -551130 & 0.89 & 17.23 & 21.32 & S & 0.000 & 111 & - & - & - & $0.04_{-0.04}^{+0.06}$ & & \\
\hline $585 \mathrm{~A}$ & 31309.3 & -551140 & 1.80 & 19.23 & 23.82 & B & 1.383 & 111 & 44.29 & -20.01 & 1.16 & $0.01_{-0.01}^{+0.04}$ & 44.30 & \\
\hline $593 \mathrm{~A}$ & 31511.2 & -551202 & 1.16 & 18.61 & 22.12 & B & 0.964 & 111 & 43.19 & -20.92 & 1.75 & $0.00_{-0.00}^{+0.01}$ & 43.19 & \\
\hline $607 \mathrm{~A}$ & 31651.2 & -551306 & 0.22 & - & 19.83 & $\mathrm{~N}$ & 0.407 & 110 & 42.87 & - & - & $0.00_{-0.00}^{+0.00}$ & 42.87 & $\mathrm{C}$ \\
\hline $608 \mathrm{~A}$ & 31349.7 & -551259 & 0.57 & 17.67 & 22.46 & $\mathrm{~N}$ & 1.019 & 100 & 43.58 & - & - & $0.14_{-0.14}^{-0.24}$ & 43.64 & 4 \\
\hline $610 \mathrm{~A}$ & 31551.8 & -551222 & 1.25 & 17.64 & 21.12 & $\mathrm{~N}$ & 0.699 & 111 & 43.10 & - & - & $8.32_{-2.45}^{-3.14}$ & 43.90 & \\
\hline $615 \mathrm{~A}$ & 31459.5 & -550357 & 1.75 & 19.15 & 22.77 & B & 1.355 & 101 & 43.99 & -21.01 & 1.46 & $0.00_{-0.00}^{+0.06}$ & 43.99 & $\mathrm{C}$ \\
\hline $615 B$ & 31500.2 & -550353 & 5.00 & 17.08 & 19.23 & $\mathrm{~N}$ & 0.096 & 011 & - & - & - & - & - & $3, \mathrm{C}$ \\
\hline $631 \mathrm{~A}$ & 31532.7 & -551200 & 0.89 & 19.65 & - & - & - & - & - & - & - & - & - & \\
\hline $632 \mathrm{~A}$ & 31624.6 & -552133 & 2.07 & 18.97 & 23.29 & $?$ & - & 000 & - & - & - & - & - & $\mathrm{C}$ \\
\hline $632 \mathrm{~B}$ & 31624.7 & -552144 & 9.08 & 19.04 & 22.93 & $\mathrm{~N}$ & 0.987 & 011 & - & - & - & - & - & \\
\hline $652 \mathrm{~A}$ & 31530.3 & -550437 & 0.52 & 18.56 & 20.75 & B & 2.710 & 111 & 45.14 & -24.66 & 1.68 & $0.00_{-0.00}^{+0.20}$ & 45.14 & 3 \\
\hline $653 \mathrm{~A}$ & 31647.4 & -551231 & 1.38 & - & 23.38 & - & - & - & - & - & - & - & - & 4, C \\
\hline $654 \mathrm{~A}$ & 31705.7 & -552718 & 3.54 & - & - & B & 2.105 & 1 - - & 45.26 & - & - & $0.33_{-033}^{+0.44}$ & 45.32 & 1 \\
\hline $656 \mathrm{~A}$ & 31326.1 & -550425 & 1.36 & 15.35 & 18.37 & $\mathrm{~N}$ & 0.166 & 111 & 42.64 & - & - & $0.91_{-0.32}^{-0.33}$ & 43.13 & \\
\hline $664 \mathrm{~A}$ & 31610.0 & -552120 & 9.50 & 18.31 & 22.60 & - & - & - & - & - & - & - & - & \\
\hline $664 \mathrm{~B}$ & 31611.0 & -552122 & 5.17 & - & - & - & - & - & - & - & - & - & - & \\
\hline $673 \mathrm{~A}$ & 31540.3 & -551221 & 0.65 & 18.76 & 22.00 & B & 1.062 & 111 & 44.03 & -21.21 & 1.45 & $0.40_{-0.19}^{+0.29}$ & 44.18 & 3 \\
\hline $675 \mathrm{~A}$ & 31624.6 & -551144 & 0.66 & 19.14 & 21.82 & B & 1.140 & 1 - - & 44.06 & -21.55 & 1.50 & $0.00_{-0.00}^{+0.06}$ & 44.06 & 3 \\
\hline $686 \mathrm{~A}$ & 31346.6 & -551149 & 0.67 & 17.08 & 19.11 & B & 1.663 & 111 & 45.44 & -25.23 & 1.58 & $0.00_{-0.00}^{+0.00}$ & 45.44 & $1,3,5$ \\
\hline $687 \mathrm{~A}$ & 31302.6 & -552608 & 4.54 & - & 18.83 & S & 0.000 & 011 & - & - & - & - & - & \\
\hline
\end{tabular}

361A: Broad absorption line quasar.

382A and 496A: Physical quasar pair, separated by 17", at $z=1.904, d_{\mathrm{A}}=143 \mathrm{kpc}$. The spectra are different, i.e., the two objects are not lensed images of the same source.

437A: Spectrum, optical, and X-ray image, and relative soft hardness ratios point undoubtedly to an $\mathrm{M}$ star as X-ray source. However, a flux ratio $f_{\mathrm{X}} / f_{\mathrm{opt}}=1.7$ is unusually high for an $\mathrm{M}$ star as the X-ray identification.

512A and 512B: Zamorani et al. (1999) identify the NELG $512 \mathrm{~B}$ with the X-ray source. The 2 mag fainter NELG 512A lies somewhat closer to the X-ray position. While both galaxies may contribute to the observed X-ray flux, we assume object 512A as the counterpart in the following.

582A: Spectrum, optical, and $X$-ray image, and relative soft hardness ratios point undoubtedly to an M star as X-ray source. However, a flux ratio $f_{\mathrm{X}} / f_{\text {opt }}=1.5$ is unusually high for an $\mathrm{M}$ star as the X-ray identification.

607A and 653A: Detected as a single X-ray source by ROSAT Zamorani et al. (1999) X404-23, X-ray source 653 is brighter and closer to X404-23 and, therefore, treated as the detected ROSAT X-ray source. The broad spectral feature at $7100 \AA$ in the optical spectrum of $607 \mathrm{~A}$ is spurious due to the zeroth order light of the neighbouring slit.

615A and 615B: Both objects, the broad emission line object $615 \mathrm{~A}$ and the narrow emission line object $615 \mathrm{~B}$, are possible counterparts to the X-ray source. We regard the fainter, but positionally better matching object $615 \mathrm{~A}$ as the counterpart.

632A: Spectrum suggests a BL-Lac object, but the object is not a radio source, classification unclear.

\subsection{Discussion of spurious matches}

Since type I AGN are a well-established class of X-ray emitters with relatively low surface density, false matches should not play any role for this object class. However, when investigating the classes of optically normal and narrow emission line galaxies, the problem of false matches has to be taken into account due to the high surface densities of these objects. To check the quality of our optical X-ray counterpart identification, we applied various tests.

First, the derived false match rate will depend on the assumed position errors, which take into account the statistical and the systematic position error (see Sect. 2.3). The difference in X-ray and optical position scaled to a $1 \sigma_{\mathrm{X}}$ position error is shown in Fig. 10a. When we assume systematic errors of 2 arcsec, the theoretical Gaussian distribution (Eq. (7))

$f\left(r_{\mathrm{n}}\right)=N_{\text {tot }} r_{\mathrm{n}} \mathrm{e}^{-\frac{1}{2} r_{\mathrm{n}}^{2}}, r_{\mathrm{n}}=\frac{r_{\mathrm{X}}-r_{\mathrm{O}}}{\sigma_{\mathrm{X}}}$

peaks significantly later than the observed distribution of the $\mathrm{X}$-ray counterparts. This is not due to the fact that we systematically accepted spurious counterparts sources, since the type I AGN distribution (shown with dotted lines in Fig. 10) is consistent with the total X-ray identification distribution. The fact that we have a large number of type I AGN allows us to estimate the actual systematic position error for our X-ray observation. The observed distribution of the type I AGN position errors can be well reproduced with a systematic error of $\sigma_{\text {syst. }}=0.7^{\prime \prime}$ (Fig. 10b). Moreover, the distribution of normalised position differences for the total sample agrees well with the theoretical distribution and with the type I AGN distribution when we apply $\sigma_{\text {syst. }}=0.7^{\prime \prime}$. 
Considering $\sigma_{\text {syst. }}=0.7^{\prime \prime}$, we calculate the false match rate by following Sutherland \& Saunders (1992) and Ciliegi et al. (2003). For every spectroscopically classified counterpart we determine the likelihood ratio $L$ by

$L=\frac{Q \exp \left(-d i s t_{\mathrm{OX}}^{2} / 2\right)}{2 \pi \sigma_{\mathrm{X}}^{2} N\left(<m_{\mathrm{R}}\right)}$,

where $L$ is the probability of finding the true optical counterpart in this position with this magnitude, relative to that of finding a similar chance background object. Hence, the reliability of a counterpart is given by $P_{\text {true }}=L /(1+L)$. $Q$ is the probability that the counterpart of the X-ray source is brighter than the limit of the optical survey and has been set here to $Q=0.5 . N\left(<m_{\mathrm{R}}\right)$ is the number density of catalogue objects of the relevant class brighter than the counterpart. For narrow emission line counterparts we assumed that about one-third of the field galaxies show line emission detectable in our spectra (Kennicutt 1992). We therefore derive the density of background objects $N\left(<m_{\mathrm{R}}\right)$ by multiplying the total number of objects $<m_{\mathrm{R}}$ in the optical catalogue by a factor 0.33 . For normal galaxies a factor of 0.67 was applied accordingly.

The total number of false matches can then be calculated by adding up all probabilities $P_{\mathrm{sp}}=1 /(1+L)$ that the counterpart is spurious. Statistically, $\sim 25 \%$ of our narrow emission line galaxy are spurious counterparts. However, after visually examining objects with low probabilities $P_{\text {true }}$, we find that in at least two cases $(422 \mathrm{~A}, 485 \mathrm{~A})$ the $\mathrm{X}$-ray position given by the source detection software is influenced by blending with another X-ray source and the counterparts are consistent with the peak of the brighter X-ray source (see Appendix C, finding chart). Taking this into account, we estimate that the false match rate is $\sim 20 \%$ for the sample of type II AGN. For the optically normal galaxies the false match rate calculated with the likelihood method is $\sim 2 \%$.

As a further quality check we plot the position error vs. WFI $R$-band magnitude. False matches would be recognisable due to different distributions of the type I and type II counterparts in this diagram. However, Fig. 11 shows that all object classes occupy the same regions in the diagram. Hence, the diagram does not indicate any serious contamination by false matches even for faint counterparts.

\subsection{Classification summary}

In total, we spectroscopically classified 140 of the optically identified X-ray sources. Details are shown in Table 4. Like in other deep surveys, e.g., Hasinger et al. (2001), the majority of the X-ray counterparts $(\sim 90 \%)$ are related to the accretion on supermassive black holes (type I and type II AGN). Furthermore, we classified a few galaxies and stars as optical counterparts (see Table 5).

\section{Properties of a core sample of the XMM-Newton Marano survey}

Our survey suffers somewhat from incomplete optical coverage of the X-ray survey area and, hence, a low identification rate over the whole area. To reach conclusions for the survey of a certain statistical significance we constrain our survey area and sample size to the central $0.28 \mathrm{deg}^{2}$ with a pn-exposure $>6 \mathrm{ks}$ and significance of detection of individual sources with $M L>10$. We refer to this as the "core sample". See Fig. 2 for the definition of

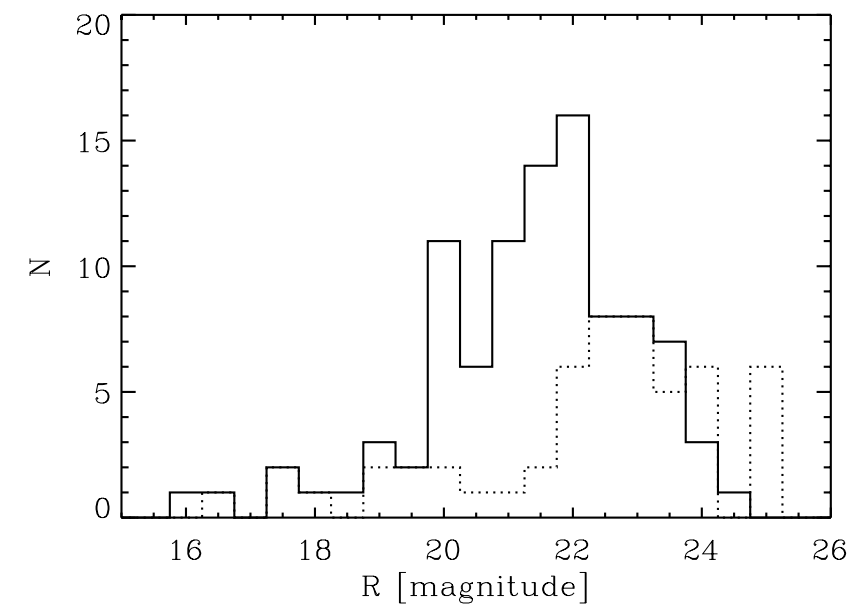

Fig. 12. WFI $R$-band magnitude histogram for the core sample in the XMM-Newton Marano Field survey. The solid line represents the $R$-magnitude distribution of the spectroscopically classified X-ray counterparts. The dashed line shows the distribution of the optically identified but not spectroscopically classified X-ray counterparts. Six X-ray sources, which have no counterpart within a $3 \sigma_{\mathrm{X}}$ error circle, are assigned to the bin at $R=25$.

the core region. Note that the maximum exposure is $35.5 \mathrm{ks}$ for the PN camera and $78 \mathrm{ks}$ for each of the MOS cameras.

The core sample contains $170 \mathrm{X}$-ray sources $(M L>10)$. No optical data are available for six out of the $170 \mathrm{X}$-ray sources which fall outside the WFI $R$-band image and are also not covered by VLT pre-images. A further six X-ray sources from the core sample have no optical detection in the WFI $R$-band image in a $3 \sigma_{\mathrm{X}}$ position error circle $\left(\sigma_{\text {syst. }}=0.7^{\prime \prime}\right) .122 \mathrm{X}$-ray sources are new detections, while 35 are spectroscopically classified ROSAT X-ray sources, and 13 are optically unidentified ROSAT sources. Out of the 140 spectroscopically classified objects in the Marano Field, 110 are associated with X-ray sources of the core sample. A summary of the properties of the core sample is given in Table 6 .

Figure 12 shows $R$-band magnitude histograms of the core sample X-ray sources that were spectroscopically classified and only optically identified, respectively. The $\left(f_{\mathrm{X}}, M L\right)$-distribution of X-ray sources in the core region is given in Fig. 13. The identification ratio of the core sample is $65 \%$. In the next subsections we always refer to this sample.

\subsection{X-ray properties}

Characterising X-ray sources only by the X-ray properties can be used to reveal and study the existence of different X-ray populations and their features. Hardness ratios (see Sect. 2.1) are the simplest tool to determine the spectral energy distribution in the X-ray regime. In Fig. 14 (HR2 vs. HR3) we only plot those X-ray sources in an X-ray colour-colour diagram that have the $H R 2$ error $\sigma_{\mathrm{HR} 2} \leq 0.3$. The different spectroscopically classified classes occupy different regions in this Xray colour-colour diagram. A noticable separation in type I and type II AGN can be recognised. This result is in agreement with Mainieri et al. (2002) and Caccianiga et al. (2004). Type II AGN spread over a much broader HR2 $(0.9>H R 2>-0.6)$. The lowest $H R 2$ values for type II AGN overlap with the highest $H R 2$ values for type I AGN. However, we do not see a large fraction of type II AGN occupying the HR2 range typical for type I AGN, as was reported by Della Ceca et al. (2004) for the 


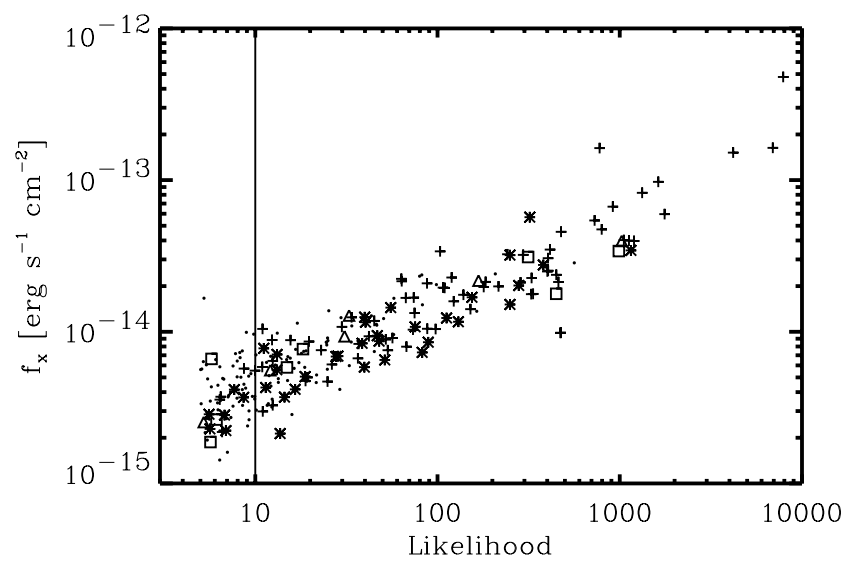

Fig. 13. Likelihood vs. $0.2-10 \mathrm{keV} X$-ray-flux of the 252 central X-ray sources (pn-detector exposure time $\geq 6 \mathrm{ks}$ ). Crosses mark type I AGN, asterisks type II AGN, triangle galaxies, and rectangles stars. Dots indicate X-ray sources with no spectroscopic classification. The vertical line indicates $M L=10$.

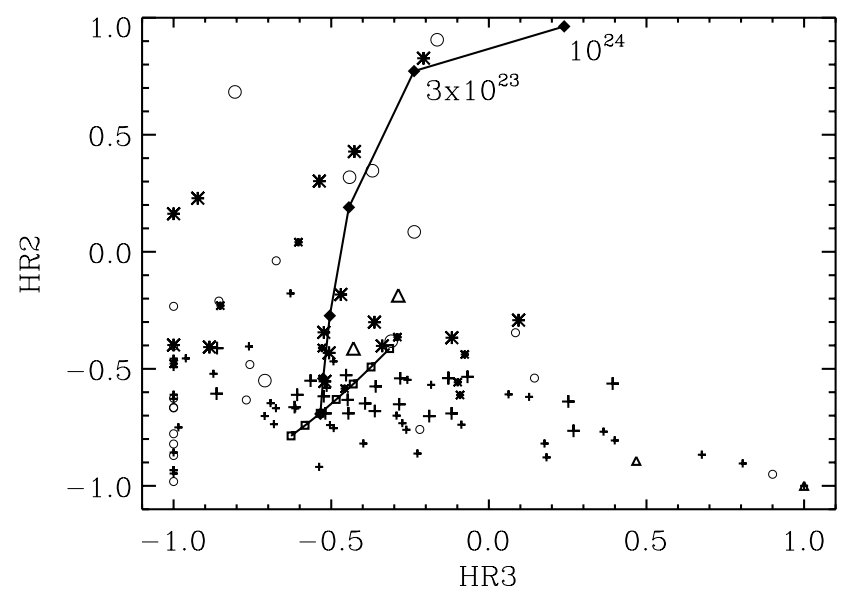

Fig. 14. X-ray diagnostic diagram based on hardness ratios. $H R 2$ is calculated by using the $0.5-2.0 \mathrm{keV}$ and $2.0-4.5 \mathrm{keV}$ band, $H R 3$ $2.0-4.5 \mathrm{keV}$ and $4.5-7.5 \mathrm{keV}$. Only X-ray sources that have $\sigma_{\mathrm{HR} 2}<0.3$ are plotted. Labels: crosses - 63 type I AGN, asterisks - 24 type II AGN, triangles -4 galaxies, and open circles -24 unidentified X-ray sources with $M L>10$. Large symbols represent objects with $\sigma_{\mathrm{HR} 3}<0.3$, small symbols $\sigma_{\mathrm{HR} 3} \geq 0.3$. The solid line with box symbols (at $H R 2 \sim H R 3 \sim-0.5)$ represents an unabsorbed power-law X-ray spectrum (corrected for galactic absorption) with different photon index $\Gamma=2.4-1.2$ in steps of 0.2 (squares). Different hydrogen column densities $\left(N_{\mathrm{H}} / \mathrm{cm}^{-2}=10^{20}, 10^{22}, 3 \times 10^{22}, 10^{23}, 3 \times 10^{23}, 10^{24}\right.$; see Sect. 4.4) are plotted as a solid line with diamonds for $\Gamma=2$ and $z=1$.

XMM-Newton bright serendipitous survey. Figure 14 shows that $H R 2$ is a good indicator for intrinsic absorption. The X-ray sources with $H R 3=-1.0$ correspond to non-detections in the $4.5-7.5 \mathrm{keV}$ band. The objects with $H R 3>0.4$ and soft $H R 2$ values have $H R 3$ errors $\geq 0.3$ (small plot symbols). Therefore, it is likely that their deviation from the typical location of sources in the $H R 2-H R 3$ diagram is caused by statistical fluctuations.

The small number of optically normal galaxies span a large range in $H R 2$. Two have $H R 2$ values that belong to the softest in the whole core sample. The other two have X-ray spectra similar to those of soft type II AGN or very hard type I AGN.

In addition to the spectroscopically classified objects, we also plot unidentified objects from the core region. Out of the 60 total unidentified X-ray sources, only 24 meet the selection criterion of $\sigma_{\mathrm{HR} 2} \leq 0.3$. Based on the rather clear separation

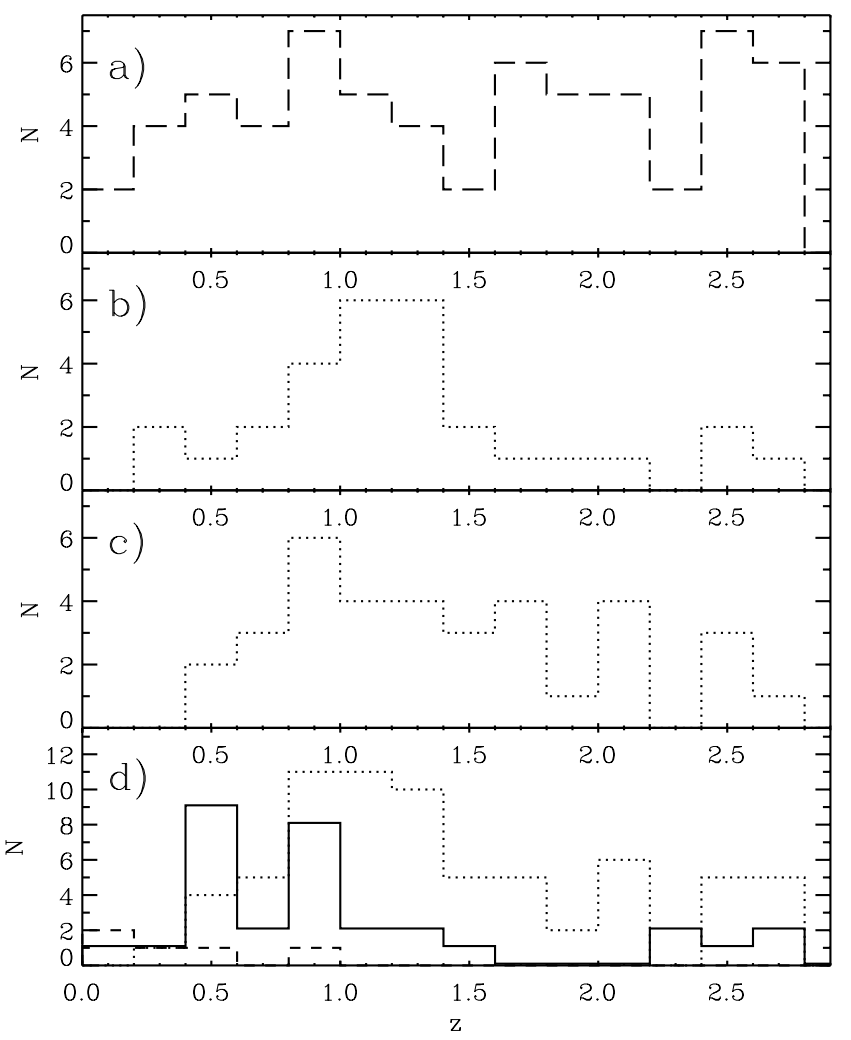

Fig. 15. Redshift distribution of AGN in the Marano Field. a) Complete sample of 64 optically selected quasars $\left(B_{\mathrm{J}} \leq 22.0\right)$ by Zitelli et al. (1992); b) ROSAT X-ray sources (29 type I AGN by Zamorani et al. 1999); c) 35 type I AGN newly detected by XMM-Newton (core region); d) redshift distribution of the core sample: dotted line: all 70 XMM-Newton detected type I AGN, solid line: 31 XMM-Newton detected type II AGN, and dashed line: 5 XMM-Newton detected optically normal galaxies.

between type I and II AGN, one may assign a likely classification to the yet unidentified sources. Among the 24 unidentified sources with reliable X-ray colours, the numbers of type I and type II AGN candidates appear to be similar.

\subsection{Redshift distribution}

Previous extensive studies of AGN in the Marano Field enable us to compare these samples with our XMM-Newton detections. The optical survey by Zitelli et al. (1992) covers $\sim 0.7 \mathrm{deg}^{2}$. The selected quasars, which are all of type I, show an almost flat distribution in redshift (Fig. 15a) up to $z=2.8$. The ROSAT $0.2 \mathrm{deg}^{2}$ survey in the field (Zamorani et al. 1999) recovered most of the optically selected quasars at redshifts up to $z \sim 1.4$ (Fig. 15b). The newly detected ROSAT AGN, with few exceptions, are type I AGN, which is expected due to the limited capability of ROSAT to detect absorbed sources.

In the $0.28 \mathrm{deg}^{2}$ core region of the XMM-survey we have detected 23 of the 29 broad emission line quasars of the optically selected sample of Zitelli et al. (1992). The detection rate of optically selected quasars remains constantly high over all redshifts. However, looking at the type I AGN newly discovered with XMM-Newton (Fig. 15c), it is apparent that the X-ray selection tends to detect quasars at lower redshifts than the optical surveys. This is particularly obvious from the redshift distribution of the ROSAT detected quasars, representing the brightest $\mathrm{X}$-ray sources in the field. But the mean redshift $z \sim 1.3$ of the 


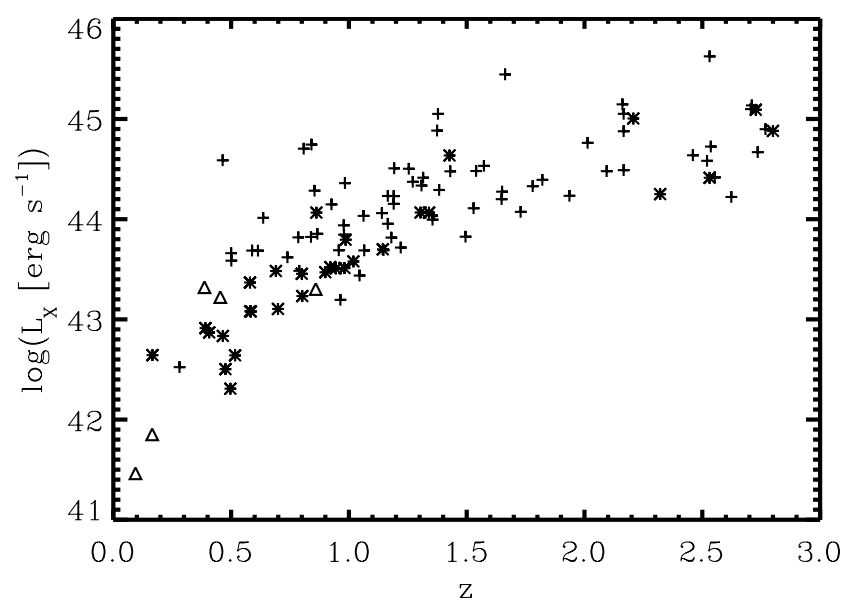

Fig. 16. Observed X-ray luminosity $(0.2-10 \mathrm{keV})$ vs. redshift. Labels: crosses - 70 type I AGN, asterisks - 30 type II AGN, and triangles 5 galaxies.

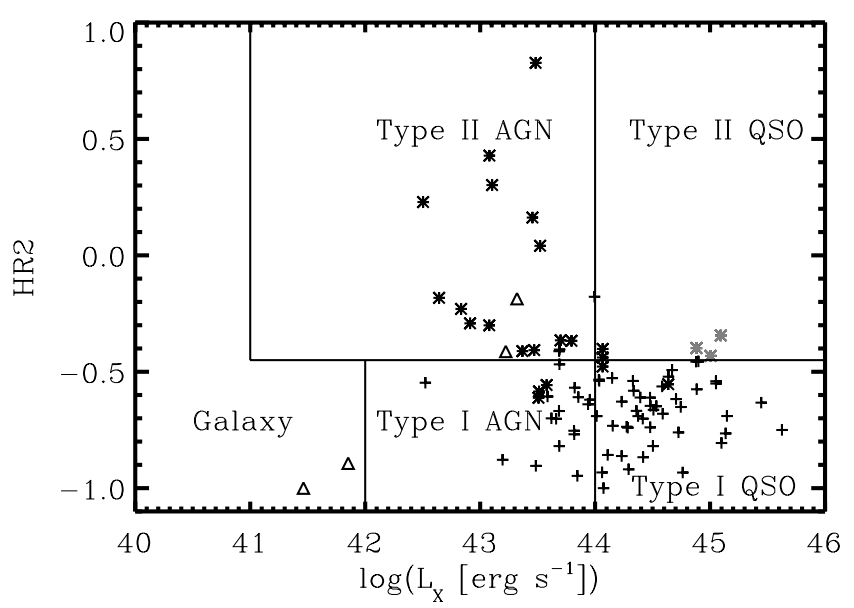

Fig. 17. Observed X-ray luminosity $(0.2-10 \mathrm{keV})$ vs. hardness ratio. $H R 2$ is calculated by using $0.5-2.0 \mathrm{keV} \& 2.0-4.5 \mathrm{keV}$ bands. Only $\mathrm{X}$-ray sources that have $\sigma_{\mathrm{HR} 2}<0.3$ are plotted. Labels: crosses 63 type I AGN, asterisks - 24 type II AGN (grey marked have $z \geq 2.0$ ), and triangles -4 galaxies. The diagram separation into different regions is based on Szokoly et al. (2004) with an adjusted threshold in the HR2value.

XMM-Newton detected type I AGN is also lower than that of the optically selected sample $z \sim 1.5$, despite the fact that the XMM-Newton-observations are deeper in terms of the surface density of quasars than the optical survey. Possible reasons for these differences are discussed in Sect. 6.

The redshift distribution according to object class of the XMM-Newton detected core sample is given in Fig. 15d. We find that almost half of the new XMM-Newton sources are classified as type II AGN with redshifts mostly below 1.0. Type I AGN extend over a wide range of redshifts with a maximum at $z \sim 0.8-1.4$. Type II AGN are comparable to type I AGN in number density at low redshifts, but are mostly found below $z=1$. Five type II quasars at $z>2.2$ have been identified. Optically normal galaxies without emission lines are found at $z<0.9$.

\subsection{Observed $X$-ray luminosity}

For the X-ray sources with measured redshifts X-ray luminosities can be computed. The coverage of the survey in redshift
- X-ray luminosity space is plotted in Fig. 16. Szokoly et al. (2004) showed that the different object classes identified in $\mathrm{X}$-ray surveys occupy different regions in a diagram of hardness ratios versus observed X-ray luminosities. In Fig. 17 we follow a scheme similar to that adopted by Szokoly et al. (2004) and consider objects with an X-ray $\operatorname{luminosity} \log \left(L_{\mathrm{X}}\right) \geq 44.0$ as quasars (QSOs). The majority of type I AGN $(\sim 70 \%)$ are actually type I QSOs. Most of type II AGN $(\sim 71 \%)$ are low X-ray luminosity objects. Only type II objects with redshifts $z \geq 2.0$ (marked in grey in Fig. 17) have X-ray luminosities of type II QSOs.

High-redshift type II QSOs with intrinsic absorption are found to be indistiguishable from non-absorbed type I AGN on the basis of their X-ray spectral hardness ratios, since the absorbed part of the spectrum is shifted out of the observable spectral window towards lower energies. This explains the emptiness of the upper right corner of Fig. 17 labelled "type II QSO", a classification that applies to low redshift objects only.

Optically normal galaxies vary clearly in X-ray luminosity. The $H R 2$-soft objects have very low $\mathrm{X}$-ray luminosities. The two $H R 2$-hard normal galaxies are found in the same region as the softest type II AGN, but are harder than type I AGN.

Szokoly et al. (2004) use $H R 2=-0.2$ as a threshold for the separation of type I and type II objects. Assuming a $\Gamma=2$, this value corresponds to a hydrogen column density $N_{\mathrm{H}} / \mathrm{cm}^{-2}=10^{22}, 10^{23}$ for $z=0.25$ and 2.1 , respectively. For their CHANDRA observation they computed $L_{X}$ in the $0.5-10 \mathrm{keV}$ band and their hardness ratio was based on the $0.5-2 \mathrm{keV}$ and $2-10 \mathrm{keV}$ bands. Because their definition of the X-ray bands differs from our study, the majority of their objects have higher hardness ratios (compared to our $H R 2)$. Furthermore, the XMM-Newton pn-detector, which was used for calculating the hardness ratios, has a higher efficiency in the $0.5-2 \mathrm{keV}$ band compared to the CHANDRA detector. Therefore, we lowered the $H R 2$ threshold from $H R 2=-0.2$ in Szokoly et al. (2004) to $H R 2=-0.45$. Our threshold corresponds to $N_{\mathrm{H}} / \mathrm{cm}^{-2}=0.54 \times 10^{22}, 1.7 \times 10^{22}$, and $5.4 \times 10^{22}$ for $\Gamma=2$ and $z=0.25,1$, and 2.1. This is about two times lower than the $N_{\mathrm{H}}$ cutoff Szokoly et al. (2004) are using.

\section{4. $N_{\mathrm{H}}$ column densities and corrected $X$-ray luminosities}

The hardness ratio diagram (Fig. 14) supports the view that the majority of type II AGN and a small fraction of type I AGN are obscured sources. Hence, the observed X-ray luminosity does not represent the intrinsic object X-ray luminosity. The significant deficit of soft photons as compared to a power-law spectrum reflects the existence of an absorbing component that is expressed by the hydrogen column density $N_{\mathrm{H}}$. For most of the sources the number of detected counts is not sufficient to extract a spectrum and fit a power-law model with $N_{\mathrm{H}}$ and the photon index $\Gamma$ as free parameters. We, therefore, applied a technique that uses the measured hardness ratios to calculate the $N_{\mathrm{H}}$ value for each source with a set of fixed power-law indices. Mainieri et al. (2002) found a mean value of $\langle\Gamma\rangle \simeq 2$ for 61 type I and type II AGN in the Lockman Hole. The majority of type I and II AGN are found in the range of $\Gamma \simeq 1.7-2.3$. The finding is confirmed by Mateos et al. (2005). They find $\langle\Gamma\rangle=1.92$ with a $\sigma=0.28$. Therefore, we use the observed pn-, mos1-, and mos2-hardness-ratios $(0.2-0.5 \mathrm{keV}, 0.5-2.0 \mathrm{keV}, 2.0-4.5 \mathrm{keV}$, and $4.5-7.5 \mathrm{keV}$ ) and performed three runs to determine $N_{\mathrm{H}}$ with the values $\Gamma=1.7,2.0$, and 2.3 for all objects.

First of all, we computed a grid of model hardness ratios for all EPIC instruments with Xspec (using the models wabs, zwabs, and powerlaw). As input the galactic absorption in 
the line of sight in the field with $N_{\mathrm{H}}=2.7 \times 10^{20} \mathrm{~cm}^{-2}$, the redshift of the object and a grid of hydrogen column densities $\left(N_{\mathrm{H}}=0, N_{\mathrm{H}}=10^{20+0.04 a} \mathrm{~cm}^{-2}, \quad a \in N_{0}, \quad a=0 . .100\right)$ is used. We then computed the $\chi^{2}$ values for the deviations of the measured hardness ratios and their model values, summed over the three instruments and the three hardness ratios $H R 1, H R 2$, and $H R 3$. The procedure was applied only to those sources that had at least five out of nine hardness ratios with $\sigma H R_{\mathrm{obs}, \mathrm{i}}<0.7$.

The $N_{\mathrm{H}}$ of a source is determined by finding the minimum of

$\chi^{2}\left(N_{\mathrm{H}}\right)=\sum_{i} \frac{\left(H R_{\mathrm{obs}, \mathrm{i}}-H R_{\text {model } \mathrm{i}}\left(N_{\mathrm{H}}\right)\right)^{2}}{\sigma H R_{\mathrm{obs}, \mathrm{i}}^{2}}$.

The models are based on the photon index $\Gamma=2$. The statistical $1 \sigma$ errors were derived from the range in $N_{\mathrm{H}}$, where $\chi^{2}<\chi_{\min }^{2}+1$ (Lampton et al. 1976). For the error calculation we also took into account an intrinsic scatter in the photon indices. Its contribution to the $N_{\mathrm{H}}$ error was measured by finding the minimum $\chi^{2}$ for each source in the grids calculated using $\Gamma=1.7$ and $\Gamma=2.3$. The resulting systematic errors were quadratically added to the statistical errors. The $N_{\mathrm{H}}$ values and the total errors are given in Table 8. Due to the uncertainties in the $N_{\mathrm{H}}$ determination we regard all values with $N_{\mathrm{H}} \leq 10^{21} \mathrm{~cm}^{-2}$ as consistent with unabsorbed spectra.

We tested our procedure by performing an individual Xspec $N_{\mathrm{H}}$-fit for the brightest type II AGN, which has sufficient X-ray data quality. The hardness ratios of $32 \mathrm{~A}(z=2.727)$ indicate the highest absorption among the brightest type II AGN. A spectral fit with wabs, zwabs, powerlaw, and $\Gamma$ as a free parameter determined $N_{\mathrm{H}_{32 \mathrm{~A}}}=(10.0 \pm 3.5) \times 10^{22} \mathrm{~cm}^{-2}$ and $\Gamma_{32 \mathrm{~A}}=(1.7 \pm 0.3)$. The best fit $N_{\mathrm{H}}$, for a fixed value of $\Gamma=1.7$, with the hardnessratio $\chi^{2}$-minimum fit is $N_{\mathrm{H}_{32 \mathrm{~A}}}=\left(7.5_{-2.8}^{+3.4}\right) \times 10^{22} \mathrm{~cm}^{-2}$. A spectral fit with a fixed $\Gamma_{32 \mathrm{~A}}=2.0$ results in $N_{\mathrm{H}_{32 \mathrm{~A}}}=(13.4 \pm 2.5) \times$ $10^{22} \mathrm{~cm}^{-2}$. The given $N_{\mathrm{H}}$ in Table 8 was performed with the hardness-ratio $\chi^{2}$-minimum fit and finds $N_{\mathrm{H}_{32 \mathrm{~A}}}=\left(14.5_{-8.2}^{+7.9}\right) \times$ $10^{22} \mathrm{~cm}^{-2}$. Hence, both fit methods give comparable results, at least for high $S N R$ sources.

Figure 18 shows the computed hydrogen column densities for type II AGN and optically normal galaxies. As expected, the majority of type II AGN shows absorption. However, $13 \%$ of the type II objects $(51 \mathrm{~A}, 132 \mathrm{~A}, 133 \mathrm{~A}$, and 607A) have absorbing column densities $N_{\mathrm{H}}<10^{21} \mathrm{~cm}^{-2}$. All of the type II AGN discovered by Mainieri et al. (2002) in the Lockman Hole have absorbed X-ray spectra. The values for optically normal galaxies range from unabsorbed to moderately absorbed. Two of the unabsorbed galaxies have low X-ray luminosities $\left(L_{\mathrm{X}}<\right.$ $10^{42} \mathrm{erg} \mathrm{s}^{-1}$ ).

The computed hydrogen column density of type II AGN object $145 \mathrm{~A}$ is the highest in our sample $\left(N_{\mathrm{H}}=10^{24} \mathrm{~cm}^{-2}\right)$. Since this corresponds to the highest value in the model grid, no reliable error estimate can be given for this object. However, the extremely high column density for this object is confirmed by the fact that it is one of the few sources detected in the $4.5-7.5 \mathrm{keV}$ EPIC images, but it is not visible in the softer bands.

Based on the hydrogen column densities, we calculated the unabsorbed (intrinsic) X-ray luminosities by computing a correction factor for the observed X-ray flux. Figure 19 shows the comparison of the absorbed (a) and unabsorbed (b) X-ray luminosity distributions. Even after correcting the X-ray luminosity, type II AGN have lower median X-ray luminosities than type I AGN, although type I and II AGN cover the same X-ray luminosity range. In contrary to the almost flat distribution of type II AGN with a median of $L_{\mathrm{X}} \sim 10^{44} \mathrm{erg} \mathrm{s}^{-1}$, type I AGN

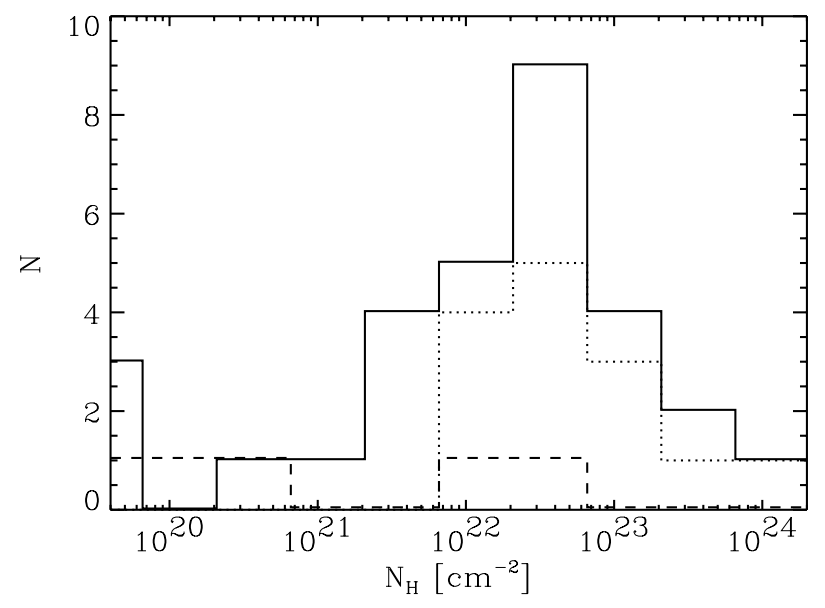

Fig. 18. Calculated intrinsic hydrogen column density histogram for the central Marano Field. The solid line shows the distribution of type II AGN that have reasonable fits in hydrogen column density. Objects with $N_{\mathrm{H}}=0$ are set to $N_{\mathrm{H}}=5 \times 10^{19} \mathrm{~cm}^{-2}$. The dotted line represents only type II AGN that show intrinsic absorption with a significance higher than $2 \sigma$ in hydrogen column density. Optically normal galaxies are plotted as a dashed line. Dotted line - 14 type I AGN; solid line - 30 type II AGN; dashed line - 5 galaxies.

show a significant peak at $L_{\mathrm{X}} \sim 10^{44.4} \mathrm{erg} \mathrm{s}^{-1}$ in observed and intrinsic X-ray luminosity. Type I AGN 5A exhibits the highest $\mathrm{X}$-ray luminosity in our sample.

The hydrogen column density as a function of the intrinsic X-ray luminosity is shown in Fig. 19c. Mainieri et al. (2002) suggest labelling the region defined by $N_{\mathrm{H}}>10^{22} \mathrm{~cm}^{-2}$ and $L_{\mathrm{X}}>$ $10^{44} \mathrm{erg} \mathrm{s}^{-1}$ as the "type II QSO region". They proposed this classification based on 61 AGN identified from XMM-Newton sources in the Lockman Hole. Following this classification our sample includes 10 type II QSOs.

In our case this region of the plot is also populated by nine type I QSOs, which formally have intrinsic hydrogen column densities $N_{\mathrm{H}}>10^{22} \mathrm{~cm}^{-2}$. However, most of these detections of intrinsic $N_{\mathrm{H}}$ have low significance; only four of all type I QSOs show absorption at the $2 \sigma$ level (see Table 7). For about half of the type II AGN, significant intrinsic absorption $\left(N_{\mathrm{H}}>2 \times \Delta N_{\mathrm{H}}\right)$ was measured, and no dependence of the absorbed fraction on luminosity is evident (Table 7).

\subsection{X-ray to optical flux ratios}

The ratio between $\mathrm{X}$-ray flux and optical flux $\left(f_{\mathrm{X}} / f_{\mathrm{opt}}\right)$ is used in former deep X-ray surveys to characterise the different X-ray emitting classes (Szokoly et al. 2004; Mainieri et al. 2002). To calculate $f_{\mathrm{X}} / f_{\text {opt }}$ values we derived optical fluxes in a band centred at $7000 \AA$ and width $1000 \AA$ using the equation $f_{\text {opt }}=10^{-0.4 R-5.759}$ (Zombeck 1990). As X-ray fluxes we used the $0.2-10 \mathrm{keV}$ values (see Sect. 2.1).

The distribution of our core sample in the $\left(R-f_{\mathrm{X}} / f_{\text {opt }}\right)$-plane is illustrated in Fig. 20. In general, type I AGN show higher $\mathrm{X}$-ray fluxes and are brighter in the $R$-band. Type II AGN are found at lower X-ray fluxes and have fainter $R$-band counterparts. Two of the optically normal galaxies have X-ray-to-optical flux ratios similar to type I and type II AGN. Another two are among the objects with lowest X-ray-to-optical flux ratios in the sample.

Our types I and II AGN show a large variety in X-ray flux and $R$-band magnitude. They are detected at X-ray fluxes from $f_{\mathrm{X}} \sim 2 \times 10^{-15}$ to $5 \times 10^{-13} \mathrm{erg} \mathrm{cm}^{-2} \mathrm{~s}^{-1}$ and in $R$-band magnitudes 


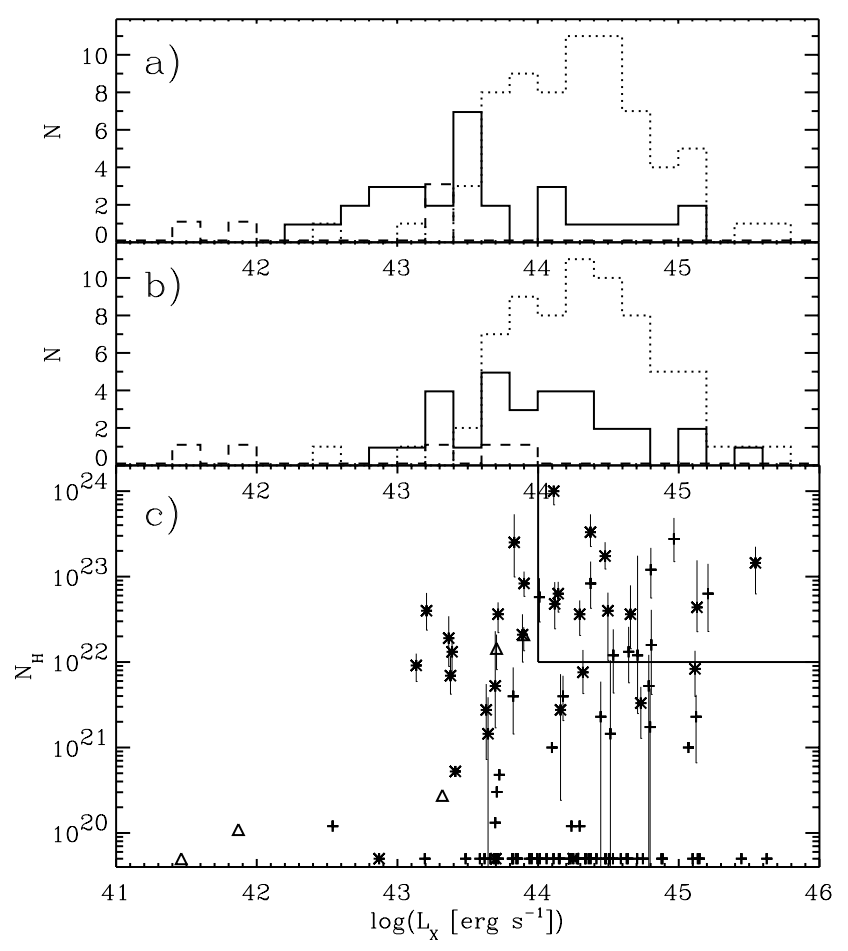

Fig. 19. a) Histogram of the absorbed (observed) X-ray luminosity $(0.2-10 \mathrm{keV})$ for the core sample. Dotted line - 70 type I AGN, solid line - 30 type II AGN, and dashed line -5 galaxies. b) Histogram of the X-ray luminosity $(0.2-10 \mathrm{keV})$ corrected for intrinsic absorption (core sample). Same notation as in a). c) Corrected X-ray luminosity $(0.2-10 \mathrm{keV})$ vs. hydrogen column density (core sample). Crosses type I AGN, asterisks - type II AGN, and triangles - galaxies. The upper right corner is suggested as being defined as the "type II QSO region" by Mainieri et al. (2002). Objects with $N_{\mathrm{H}}=0$ are set to $N_{\mathrm{H}}=5 \times 10^{19} \mathrm{~cm}^{-2}$.

from $R \sim 18$ down to the detection limit of $R \sim 24$. There is one type I AGN (191A) at an unusually low value $f_{\mathrm{X}} / f_{\text {opt }}=0.04$, a factor of 100 below the mean value of type I AGN. The highest ratio in X-ray to optical flux is found for the type II AGN object $39 \mathrm{~A}$ with $f_{\mathrm{X}} / f_{\mathrm{opt}}=42.5$.

Most of the stars are found at star-typical $f_{\mathrm{X}} / f_{\mathrm{opt}}<0.05$ with $R<17$. Nevertheless, we also detected two M stars with $f_{\mathrm{X}} / f_{\text {opt }} \sim 1$ and $R \sim 21.5$. Unidentified sources with WFI $R$-band data are added to Fig. 20. We plotted unidentified objects that have no detection in the $R$-band catalogue at $R=24$.

\subsection{Optical-to-near-IR colours}

Figure 21 shows that type II AGN tend to be fainter and redder in the optical window than type I AGN. This figure shows a general trend for both type I and type II AGN to become redder for fainter $R$-magnitudes. The lack of faint blue objects can be explained by the $K$-band detection limit of $K=20$, where we lose completeness (see Fig. 6). However, the lack of bright red objects cannot be caused by any detection bias. The type II AGN have redder $R-K$ colours, although with some overlap with the reddest type I AGN in the sample. These trends can be explained by an increasing contribution of the host galaxies for fainter type I AGN and type II AGN. The faint $R$-magnitudes and high $R-K$ values indicate higher optical obscurations in type II AGN. This is in agreement with previous X-ray studies of these objects that revealed a significantly higher ratio of absorbed to unabsorbed objects compared to type I AGN.

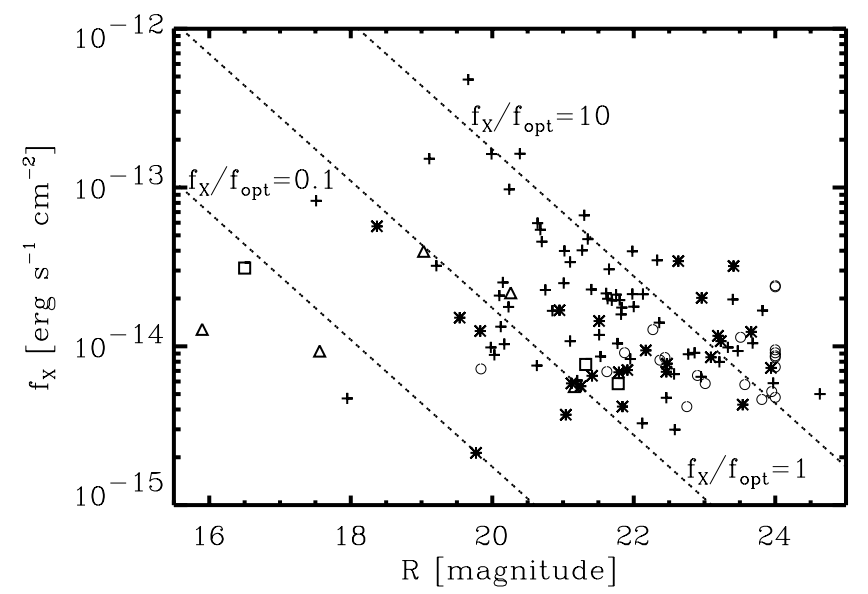

Fig. 20. Observed X-ray flux $(0.2-10 \mathrm{keV})$ vs. $R$-band magnitude. Dashed lines indicate the $f_{\mathrm{X}} / f_{\text {opt }}$ values $0.1,1.0$, and 10 . Labels: crosses -63 type I AGN, asterisks - 25 type II AGN, triangles -5 galaxies, rectangles -3 stars, and circles -21 spectroscopically unidentified sources ( $R$-band lower limits are plotted as circles at $R=24$ ). Note that for a few objects of the core sample no WFI $R$-magnitudes were available and therefore these are not plotted here.

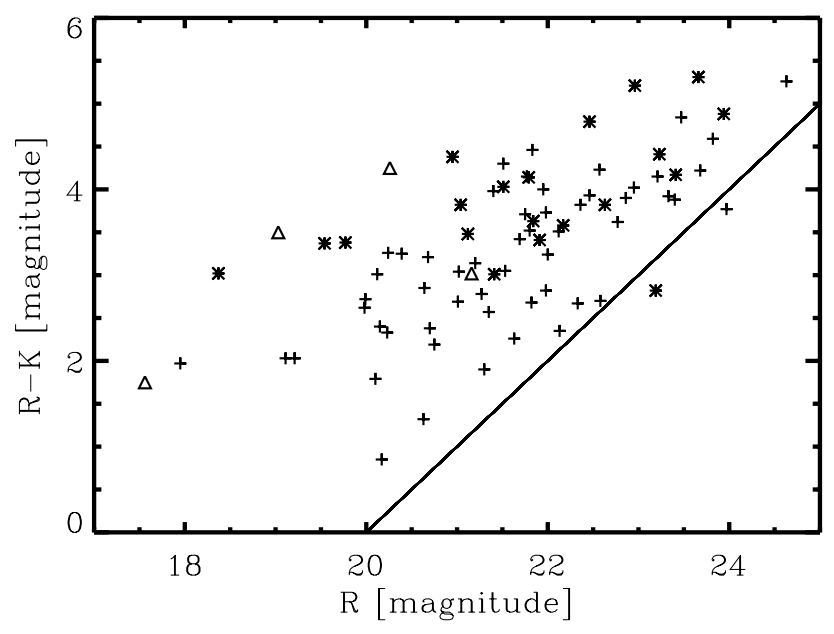

Fig. 21. Colour magnitude diagram of spectroscopically classified X-ray sources. Labels: crosses - 56 type I AGN, asterisks 20 type II AGN, and triangles -4 galaxies. Only objects with detections in both $R$ and $K$ are included. The absence of blue, faint objects (below the solid line) is due only to the $K$-magnitude limit.

In our survey type I and type II objects do not separate as clearly as seen in a similar plot in Mainieri et al. (2002). Therefore, we checked whether the reddest and faintest type I objects are reliably classified. The most extreme type I objects are $69 \mathrm{~A}, 84 \mathrm{~A}$, and 585A. Their optical spectra clearly show broad emission lines, but their continua are redder than typical type I spectra. In addition, object 585A shows only weak emission lines.

The optically normal galaxies are brighter in $R$-magnitudes than the typical type II AGN, but show similar $R-K$. All classified galaxies have $R<21.5$. The majority of type II AGN and galaxies have $R-K>3 \mathrm{mag}$, consistent with the spectrum of the host galaxy being the dominating component. A considerable fraction of type I AGN also have red $R-K$ colours. These are mostly low luminosity objects, where the host galaxy may also dominate the optical continuum. 


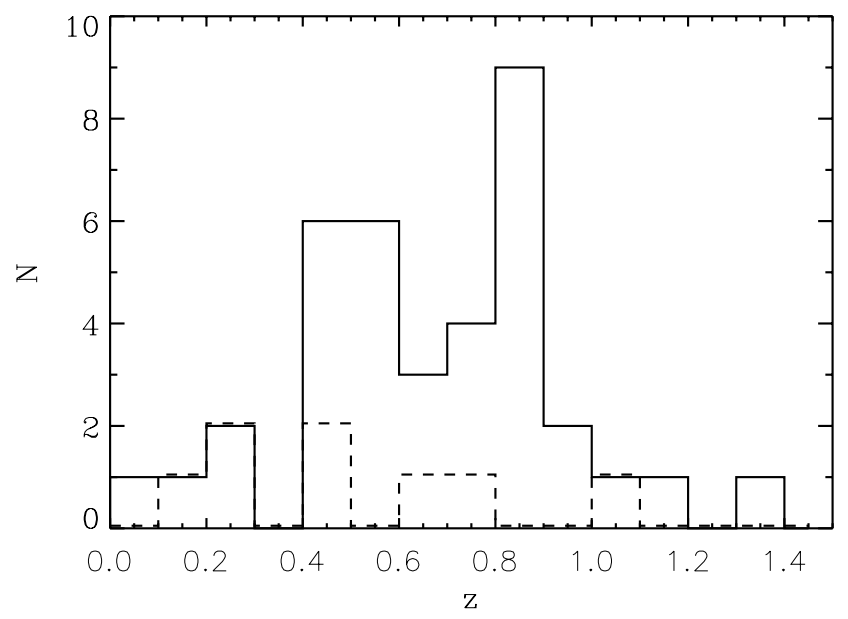

Fig. 22. Redshift distribution of the additional objects. Labels: solid line - 37 NELGs, and dotted line -8 optically normal galaxies.

\section{Additional objects}

In Table D.1 (Online Material, Appendix D) we list objects in the Marano Field that are not related to X-ray sources. These objects were spectroscopically investigated since slit positions on the multi-object spectroscopy masks were still available. The random selection of these additional objects, which follow a similar $R$-magnitude distribution as the sample of X-ray selected type II objects, is useful to investigate the redshift distribution of the field galaxies in the Marano Field.

Figure 22 illustrates the redshift distribution of the additional non-X-ray emitting objects in the Marano Field. Narrow emission line galaxies (NELG) outnumber normal galaxies substantially. This is due to the fact that a large fraction of the spectra without emission lines did not have sufficient SNR to determine a redshift. There is a non-X-ray emitting NELG peak at $z=0.5-0.9$. At $z>1.0$ the detection of NELG and normal galaxies drops dramatically.

Despite the low number of objects, from Figs. 22 and 15d we can compare the redshift distributions between X-ray emitting type II AGN and non-X-ray emitting NELG. Both populations show more or less the same distribution up to $z=1.2$. The two groups peak at $z=0.5-0.9$.

The similar redshift distributions could mean that the X-ray emitting NELGs are drawn from the same population of galaxies as the control sample. On the other hand, the similar redshift distribution could also be due to a large fraction of false matches of NELGs to our X-ray sources. A detailed discussion of the number of false matches is given in Sect. 3.2 and shows that false matches make only a minor contribution to our sample of narrow line AGN.

\section{Discussion}

\subsection{Type I AGN}

In Sect. 4.2 we showed that the X-ray selected type I AGN peak at lower redshifts than the optically selected sample. It is interesting to see whether this difference in the redshift distributions is due to a redshift dependence of the QSO spectral energy distributions (SED). As a parameter which characterises the SED, we calculated the optical to X-ray broad band spectral index $\alpha$ OX between the UV luminosity density at $2500 \AA$ and the X-ray luminosity density at $1 \mathrm{keV}$ (see Sect. 3).

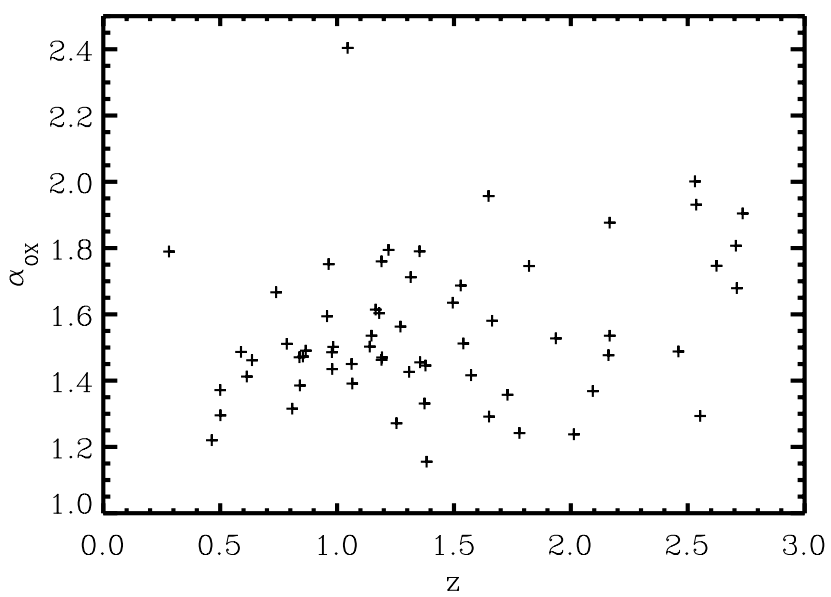

Fig. 23. UV to X-ray spectral index $\alpha_{\mathrm{OX}}$ vs. redshift for type I AGN in the core sample.

No significant correlation of $\alpha_{\mathrm{OX}}$ with redshift (Fig. 23) or $\mathrm{X}$-ray luminosity can be found. However, there is a very significant $\left(P_{\text {NULL }}<10^{-6}\right)$ correlation of the optical luminosity and $\alpha_{\mathrm{OX}}$ (Fig. 24). This correlation has been found in various samples observed with EINSTEIN (Avni \& Tananbaum 1986), ROSAT (e.g., Green et al. 1995; Lamer et al. 1997), and CHANDRA (e.g., Steffen et al. 2006). For a large sample of optically selected AGN, Steffen et al. (2006) computed the bivariate linear regression coefficients of $\alpha_{\mathrm{OX}}$ as a function of $\log \left(L_{\mathrm{opt}}\right)$ and $z$. They find that $\alpha_{\mathrm{OX}}$ is correlated with $\log \left(L_{\mathrm{opt}}\right)$, but find no significant correlation with $z$ (see also Avni \& Tananbaum 1986 for similar results on earlier EINSTEIN data).

If $\alpha_{\mathrm{OX}}$ and optical luminosity are correlated, a non-linear relation between X-ray luminosity and optical luminosity is expected. Therefore, we plotted the X-ray luminosities versus the optical luminosity densities (Fig. 25) and computed the linear regression coefficients between $\log \left(L_{\mathrm{X}}\right)$ and $\log \left(l_{2500 \AA}\right)$. Both variables $\log \left(L_{\mathrm{X}}\right)$ and $\log \left(l_{2500 \AA}\right)$ are measured quantities and neither of them can be regarded as the independent or dependent variable. We used the ordinary least-squares (OLS) bisector algorithm as described by Isobe et al. (1990), which is symmetric regarding the choice of independent and dependent variable.

We find a best-fit regression $\log \left(L_{\mathrm{X}}\right)=(0.84 \pm 0.08) \times$ $\log \left(l_{2500 \AA}\right)+(19.37 \pm 2.23)$. This slope is marginally $(2 \sigma$ significance) flatter than the $\beta=1.0$ expected for a linear $L_{\mathrm{X}}-l_{\mathrm{opt}}$ relation. With the same method Steffen et al. (2006) find a slightly flatter slope $\beta=0.721 \pm 0.011$.

A correlation flatter than $\beta=1$ implies that the optical luminosities in the sample are spread over a wider range of values than the X-ray luminosities. This might explain the abovementioned discrepancies of the redshift distributions of X-ray and optically selected QSOs. The low luminosity objects are more likely to be detectable in X-rays, while the highest luminosity objects are relatively more luminous in the optical, and therefore detectable at higher redshifts in optical surveys.

\subsection{Type II AGN}

Figure $15 \mathrm{~d}$ shows that most type II AGN are found at redshifts $z \sim 0.5-0.9$, with a few objects at $z>2.2$. No type II objects were identified in the redshift range $z \sim 1.5-2.2$. The interesting question is whether this redshift gap reflects the intrinsic distribution or is due to an observational bias. 


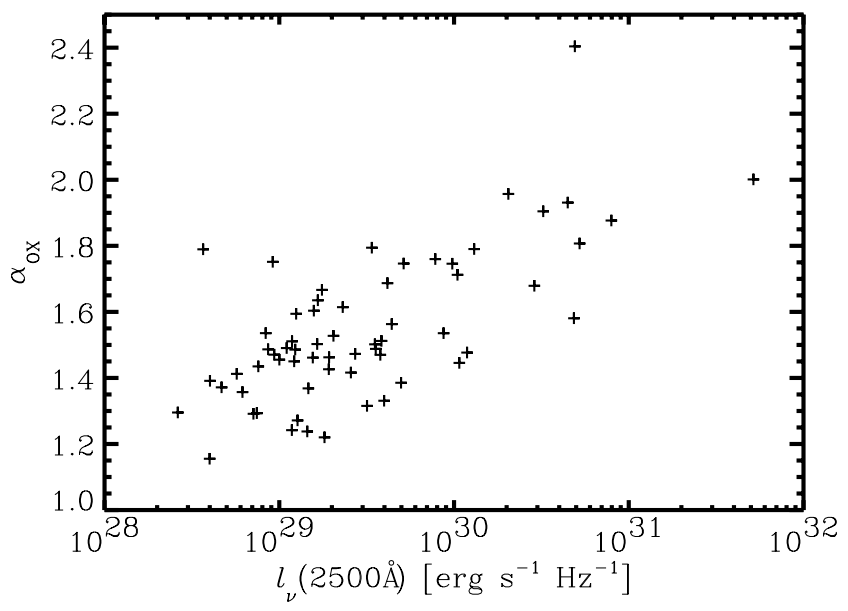

Fig. 24. UV to X-ray spectral index $\alpha_{\mathrm{OX}}$ vs. optical luminosity density at $2500 \AA$ for type I AGN in the core sample.

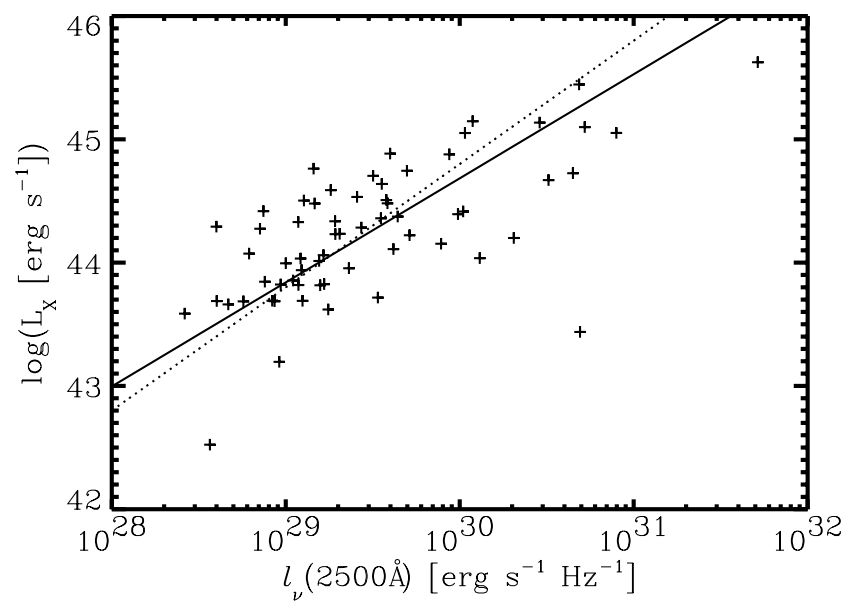

Fig. 25. Observed $0.2-10 \mathrm{keV}$ X-ray luminosity vs. optical luminosity density at $2500 \AA$ for type I AGN in the core sample (crosses). The solid line indicates the best-fit linear regression with a slope $\beta=0.84$. The dotted line with slope $\beta=1$ marks a linear $L_{\mathrm{X}}-l_{\mathrm{opt}}$ relation.

Type II AGN show only narrow emission lines and the optical continuum radiation is dominated by the host galaxy. The spectroscopical classification of X-ray sources relies on emission lines like Ly- $\alpha$, C IV, C III, Mg II, O II, and others. Analysis of the optical spectra of type II AGN with $z>0.6$ in our sample, in Szokoly et al. (2004), and in Caccianiga et al. (2004) indicates that almost all objects show no $\mathrm{Mg}$ II emission lines. Only in a few cases marginal Mg II emission is recognised. Effectively, no strong spectral features are present in the wavelength range between $1909 \AA$ (C III]) and $3727 \AA$ ([O II]).

Since the UV host galaxy continuum is usually very faint, the detection of type II AGN in optical imaging is already hampered by the lack of a strong UV continuum and emission lines, if the imaging bandpass falls into this rest frame range. For $R$-band imaging (5800-7300 $\AA$ ) this is the case for the redshift range $z \sim 1.0-2.0$. Our multi-object spectroscopy covers a useful range of $\sim 4000-9000 \AA$. This range is reduced, if an object is not centred on the mask, but offset from the centre in the dispersion direction. Hence, in many cases only one emission line would be detectable for type II AGN in the redshift range $\sim 1.0-2.0$. With a low $S N R$ spectrum this would usually not be sufficient for a clear spectroscopical classification. For a few sources, which were undetected or very faint in the $R$-band images, we were able to position a MXU slit using the $K$-band counterparts (e.g., 20A, 63A, and 463A). In all of these cases a high redshift type II AGN could be identified.

Figure 14 supports the assumption that a large fraction of unidentified sources consists of type II AGN. Above $H R 2=-0.1$ only type II AGN are found as counterparts for identified X-ray sources. In this region $50 \%$ of the X-ray sources are not classified and are expected to be type II AGN. Furthermore, Fig. 20 gives more evidence that the unidentified sources overlap with the type II AGN region. A ratio $f_{\mathrm{X}} / f_{\text {opt }}>0.5$ is clearly a sign of AGN activity, since normal galaxies and stars usually have $f_{\mathrm{X}} / f_{\text {opt }}<0.05$. Both type II AGN and unidentified objects are found at AGN-typical $f_{\mathrm{X}} / f_{\mathrm{opt}}$, with low X-ray fluxes and faint optical counterparts.

We conclude that the majority of the unidentified X-ray sources are likely to consist of type II AGN, most of them presumably with redshifts $z>1$. Therefore, the intrinsic redshift distribution of the type II AGN is uncertain. The number of unidentified sources is sufficient to fill the observed gap between $z \sim 1.0-2.0$.

\subsection{X-ray bright optically normal galaxies}

Five objects in the core sample have been spectroscopically classified as galaxies without any emission lines. The soft X-ray radiation of the low X-ray luminosity galaxies (objects 120A, 241A) can be explained by a halo of X-ray emitting hot gas around elliptical galaxies (Sarazin 1997; White \& Davis 1998). Three optically normal galaxies have X-ray-to-optical flux ratios and X-ray luminosities typically found for AGN. Objects of this type have been named X-ray bright optically normal galaxies (XBONGs; Comastri et al. 2002). The objects 8A, 49A, and 204A have intermediate redshifts $(0.3<z<0.9)$ and fairly high X-ray luminosities $\left(\log \left(L_{\mathrm{X}}\right)>43\right)$, rather hard X-ray spectra, and faint optical counterparts $(R>19)$. The high X-ray luminosities and $f_{\mathrm{X}} / f_{\text {opt }} \sim 1$ indicate active nuclei. The computed hydrogen column densities show moderate absorption.

The properties of our XBONGs are in agreement with other studies (Silverman et al. 2005; Severgnini et al. 2003). Even though different scenarios are discussed, the nature of XBONGs still remains unclear (Brandt \& Hasinger 2005). Comastri et al. (2002) assume that the non-detection of optical emission lines and the hard X-ray colours are due to a heavily absorbed AGN embedded in a galaxy whose X-ray emission is due to a scattered/reprocessed nuclear component. The three XBONGs do not show harder $H R 2$ values than type II AGN and also do not have the highest column densities of the AGN sample. Hence, a heavily absorbed AGN without scattered/reprocessed X-ray radiation is ruled out. Scattered or reprocessed radiation is necessary to explain the X-ray and optical observations. Following Komossa et al. (1998), the observed X-ray luminosity of a scattered emission by a warm reflector is only a hundredth or thousandth of the intrinsic X-ray luminosity. For our objects with observed $\log \left(L_{X}\right) \sim 43-44$, this would require intrinsic X-ray luminosities of $\log \left(L_{X}\right) \sim 45-47$, which would make them by far the most luminous X-ray emitters in the sample.

Another possible explanation for these objects is given by Severgnini et al. (2003). They studied three low redshift XBONGs with $\log \left(L_{\mathrm{X}}\right) \sim 42-43$ and find $N_{\mathrm{H}}$ values similar to our sample. In their interpretation the faint emission lines of an obscured or unobscured AGN up to an intrinsic $\log \left(L_{\mathrm{X}}\right) \sim 43$ can be overwhelmed by a host galaxy with an absolute magnitude $M_{\mathrm{R}} \geq-22$. However, XBONGs $8 \mathrm{~A}$ and $49 \mathrm{~A}$ are not consistent with the scenario mentioned by Severgnini et al. (2003). The 
AGN of 49A with an intrinsic $\log \left(L_{\mathrm{X}}\right)=43.7$ should be optically too bright to be hidden by a galaxy of $M_{\mathrm{R}}=-21.8$. Moreover, source $8 \mathrm{~A}$, which is also detected as a radio source (Gruppioni et al. 1999), has an intrinsic $\log \left(L_{X}\right)=43.9$. Therefore, it is more $\mathrm{X}$-ray luminous by almost one order of magnitude than the examples in Severgnini et al. (2003). The absolute magnitude of the host galaxy is $M_{\mathrm{R}}=-22.6$, but an optically much brighter galaxy is needed to hide the emission lines of such a powerful AGN. By adding a template type I spectrum to the measured spectra of objects $8 \mathrm{~A}$ and $49 \mathrm{~A}$, we estimated that any hidden type I AGN in these objects would have $\alpha_{\text {OX }}$ values of $\sim 0.8$ or less.

Regarding the X-ray and optical colours, our XBONGs are very similar to type II AGN. Therefore, it is likely that these sources have a narrow line type II spectrum, which is intrinsically weak or dust absorbed and not detected above the continuum of the host galaxy. This result is consistent with Caccianiga et al. (2004), who state that more accurate re-observation (high resolution data and/or better spectral coverage) of hard X-ray emitting galaxies will reveal narrow emission lines and, therefore, their real AGN nature. Severgnini et al. (2003) also claim possible misclassification of type II AGN as XBONGs. However, some of these objects are still classified as XBONGs after a high resolution observation with better spectral coverage.

As for type II AGN, the observed redshift distribution of XBONGs could be due to an observational bias. XBONGs are found with brighter $R$-magnitudes than typical type II AGN up to $z=1$. But missing emission line features make them optically even more difficult to identify than type II AGN. For a reliable classification they have to be optically brighter, thus limiting their maximum redshift.

\subsection{Stars}

In the Marano Field survey $7 \%$ of the X-ray sources are identified with galactic stars. As in other deep surveys, these are typically $\mathrm{G}, \mathrm{K}$, and $\mathrm{M}$ stars, whose X-ray emission is caused by magnetic activity (Brandt \& Hasinger 2005). Two sources classified as stars have $f_{\mathrm{X}} / f_{\text {opt }} \sim 1$ (Fig. 20), which is more typical for AGN. However, we carefully reanalysed the X-ray colours, optical properties, and the optical image. Apart from the unusally high $f_{\mathrm{X}} / f_{\text {opt }}$ all existing evidence suggests $\mathrm{M}$ stars as reliable counterparts for both X-ray sources.

\section{Conclusions}

With a total of $120 \mathrm{ks}$ good observation time we detect 328 X-ray sources. Among 140 spectroscopic classifications of 187 optical counterparts (in a $3 \sigma_{\mathrm{X}}$ position error with $\sigma_{\text {syst. }}=$ $0.7^{\prime \prime}$ ) to $328 \mathrm{X}$-ray sources (not completely covered by optical data), we find 89 broad emission line objects, 36 narrow emission line objects, 6 galaxies, and 9 stars. In the central region of the Marano Field we reach an identification completeness of $65 \%$.

While the redshift distribution of the optically selected QSOs in the field is basically flat up to $z \simeq 3$, the distribution of the $\mathrm{XMM}-$ Newton sources peaks at $z \simeq 1$. Using our sample of XMM-Newton sources classified as type I AGN, we investigate possible causes for this tendency of deep X-ray surveys to discover faint populations at comparably low redshifts. We find no significant correlation of the optical to X-ray SED slope $\alpha_{\mathrm{OX}}$ with redshift. As is widely reported in the literature, $\alpha_{\mathrm{OX}}$ is tightly correlated with optical luminosity. A different representation of this correlation is the non-linear dependency $L_{\mathrm{X}}\left(l_{\mathrm{opt}}\right)$. The best-fit regression $\log \left(L_{\mathrm{X}}\right)=0.84 \times \log \left(l_{2500 \AA}\right)+19.36 \mathrm{im}-$ plies that the optical luminosities in a typical sample spread over a wider range than the X-ray luminosities. Therefore, the less luminous objects of the population are more easily detected in $\mathrm{X}$-rays than in the optical. On the other hand, an increase of optical luminosity is, on average, not accompanied by a proportional increase in X-ray luminosity. Hence, the luminous (and more distant) objects are detected more efficiently in optical surveys.

In the core region of the field we classified 31 new type II AGN. Most of them are found at redshifts $z<1.5$; additionally we find five high redshift type II AGN at $z>2.2$. Fifteen objects can be classified as type II QSOs with intrinsic X-ray luminosities $L_{\mathrm{X}}>10^{44} \mathrm{erg} \mathrm{s}^{-1}$.

We show that the optical identification of type II AGN is very difficult in the redshift range $z=1-2$ due to the absence of suitable emission lines in the optical window. Therefore, their intrinsic redshift distribution remains unclear. We demonstrate that the use of $K$-band data for MXU slit positioning reveals type II AGN or XBONGs that would have likely been missed in $R$-band images. The X-ray selected type II AGN have a very similar redshift distribution to non-X-ray emitting narrow emission line galaxies, which have been spectroscopically classified in the same field as a control sample.

The intrinsic hydrogen column densities, as derived from $\mathrm{X}$-ray hardness ratios, show that the fraction of absorbed X-ray sources is much higher for type II AGN than for type I. Nevertheless, we find a few unabsorbed type II AGN and some evidence for absorption in high redshift type I AGN. However, due to the faintness of the sources, the significance of absorption in the individual type I AGN is low. Furthermore, at high redshifts statistical fluctuations in the X-ray spectrum can lead to high values of spuriously measured $N_{\mathrm{H}}$ values (e.g., Akylas et al. 2006). If we only include $2 \sigma$ detections of intrinsic $N_{\mathrm{H}}$ in our analysis, only 4 absorbed type I AGN remain and no dependency of absorbed fraction on redshift is obvious. In the CHANDRA data from Chandra Deep Field South (CDFS), Tozzi et al. (2003) find hints of an increase of absorbed fraction with redshift. However, these data probably also suffer from the uncertainties mentioned above. Using XMM data in the same field, Dwelly \& Page (2006) find little evidence that the absorption distribution is dependent on either intrinsic X-ray luminosity or redshift. Our type I and type II AGN cover the same range in absorption corrected X-ray luminosity. However, the mean corrected X-ray luminosity is smaller for type II AGN than for type I AGN.

Three of the XMM-Newton classifications are X-ray bright optically normal galaxies (XBONGs), which show X-ray luminosities typical for AGN, but no optical emission lines. Their X-ray luminosities of $\log \left(L_{\mathrm{X}}\right) \sim 43-44$ are comparable to the mean type II AGN X-ray luminosity. They do not show harder $\mathrm{X}$-ray spectra and do not reveal higher hydrogen column densities than the average type II AGN. We conclude that the objects are very similar to type II AGN. However, their narrow emission lines are not detected, since they are either intrinsically weak or obscured by dust.

Acknowledgements. Mirko Krumpe is supported by the Deutsches Zentrum für Luft- und Raumfahrt (DLR) GmbH under contract No. FKZ 50 OR 0404. Georg Lamer acknowledges support by the Deutsches Zentrum für Luft- und Raumfahrt (DLR) GmbH under contract No. FKZ 50 OX 0201.

\section{References}

Alexander, D. M., Bauer, F. E., Brandt, W. N., et al. 2003, AJ, 125, 383 Akylas, A., Georgantopulos, I., Georgakakis, A., Kitsionas, S., \& Hatziminaoglou, E. 2006, A\&A, in press 
Avni, Y, \& Tananbaum, H. 1986, ApJ, 305, 83

Brandt, W. N., \& Hasinger, G. 2005, ARA\&A, 43, 827

Caccianiga, A., Severgnini, P., Braito, V., et al. 2004, A\&A, 416, 901

Cash, W. 1979, AJ, 228, 939

Ciliegi, P., Zamorani, G., Hasinger, G., et al. 2003, A\&A, 398, 901

Comastri, A., Mignoli, M., Ciliegi, P., et al. 2002, AJ, 571, 771

Della Ceca, R., Maccacaro, T., Caccianiga, A., et al. 2004, A\&A, 428, 383

Dwelly, T., \& Page, M. J. 2006, MNRAS, 372, 1755

Francis, P. J., Hewett, P. C., Foltz, C. B., et al. 1991, ApJ, 373, 465

Green, P. J., Schartel, N., Anderson, S. F., et al. 1995, ApJ, 450, 51

Green, P. J., Silverman, J. D., Cameron, R. A., et al. 2004, ApJS, 150, 43

Gruppioni, C., Mignoli, M., \& Zamorani, G. 1999, MNRAS, 304, 199

Gruppioni, C., Zamorani, G., de Ruiter, H. R., et al. 1997, MNRAS, 286, 470

Hasinger, G., Burg, R., Giacconi, R., et al. 1998, A\&A, 329, 482

Hasinger, G., Altieri, B., Arnaud, M., et al. 2001, A\&A, 365, 45

Horne, K. 1986, PASP, 98, 609

Isobe, T., Feigelson, E. D., \& Akritas, M. G. 1990, ApJ, 364, 104

Kennicutt, C. K. 1992, ApJ, 388, 310

Komossa, S., Schulz, H., \& Greiner, J. 1998, A\&A, 334, 110

La Franca, F., Fiore, F., Vignali, C., et al. 2002, ApJ, 570, 100

Lamer, G., Brunner, H., \& Staubert, R. 1997, A\&A, 327, 467

Lampton, M., Margon, B., \& Bowyer, S. 1976, AJ, 208, 177

Lehmann, I., Hasinger, G., Schmidt, M., et al. 2001, A\&A, 371, 833
Mainieri, V., Bergeron, J., Hasinger, G., et al. 2002, A\&A, 393, 425

Marano, B., Zamorani, G., \& Zitelli, V. 1988, MNRAS, 232, 111

Mateos, S., Barcons, X., Carrera, F. J., et al. 2005, A\&A, 444, 79

Mignoli, M., \& Zamorani, G. 1998, The Young Universe, ASP Conf. Ser., 146, 80

Oke, J. B., \& Gunn, J. E. 1983, AJ, 266, 713

Osborne, J. 2001, SSC-LUX-TN-0059 (issue3), http: - -xmmssc-www . star.

le.ac.uk/pubdocs/SSC-LUX-TN-0059_3.ps.gz

Rosati, P., Della Ceca, R., Norman, C., \& Giacconi, R. 1998, ApJ, 492, L21

Sarazin, C. L. 1997, ASP Conf. Ser., 116, 375

Severgnini, P., Caccianiga, A., Braito, V., et al. 2003, A\&A, 406, 483

Silverman, J. D., Green, P. J., Barkhouse, W. A., et al. 2005, ApJ, 618, 123

Steffen, A. T., Strateva, I., Brandt, W. N., et al. 2006, ApJ, 131, 2826

Sutherland, W., \& Saunders, W. 1992, MNRAS, 259, 413

Szokoly, G. P., Bergeron, J., Hasinger, G., et al. 2004, ApJS, 155, 271

Teplitz, H. I., Collins, N. R., Gardner, J. P., et al. 2003, ApJS, 146, 209

Tozzi, P., Gilli, R., Mainieri, V., et al. 2006, A\&A, 451, 457

Vanden Berk, D. E., Richards, G. T., Bauer, A., et al. 2001, AJ, 122, 549

White III, R. E., \& Davis, S. D. 1998, ASP Conf. Ser., 136, 299

Worsley, M. A., Fabian, A. C., Bauer, F. E., et al. 2005, MNRAS, 357, 1281

Zamorani, G., Mignoli, M., Hasinger, G., et al. 1999, A\&A, 346, 731

Zitelli, V., Mignoli, M., Zamorani, G., et al. 1992, MNRAS, 256, 349

Zombeck, M. V. 1990, Handbook of Astronomy (Cambridge University Press) 
M. Krumpe et al.: The XMM-Newton survey in the Marano field. I., Online Material $p 1$

\section{Online Material}




\section{Appendix A: X-ray source list of the XMM-Newton Marano Field mosaic}

\section{A.1. Explanation of table columns (detailed description in Sect. 2.1)}

(1) ID

Classification number of the XMM-Newton X-ray source in the Marano Field.

(2) Name XMMU J...

XMMU source name of the XMM-Newton X-ray source.

(3) $C R\left[\mathrm{ks}^{-1}\right]$

Added count rates from all 3 EPIC cameras.

(4) $M L$

Maximum detection likelihood.

(5) $\sigma_{\mathrm{X}}[\operatorname{arcsec}]$

Position error of the X-ray source. $\sigma_{\mathrm{X}}=\sqrt{\sigma_{\text {stat. }}^{2}+\sigma_{\text {syst. }}^{2}}, \sigma_{\text {syst. }}=0.7^{\prime \prime}$, see Sects. 2.3 and 3.2.

(6) HR1

PN-detector hardness ratios with errors in the $0.2-0.5 \mathrm{keV}$ and $0.5-2.0 \mathrm{keV}$ band.

(7) $H R 2$

PN-detector hardness ratios with errors in the $0.5-2.0 \mathrm{keV}$ and $2.0-4.5 \mathrm{keV}$ band.

(8) HR3

PN-detector hardness ratios with errors in the $2.0-4.5 \mathrm{keV}$ and $4.5-7.5 \mathrm{keV}$ band.

(9) flux $\left[10^{-14} \mathrm{erg} \mathrm{s}^{-1} \mathrm{~cm}^{-2}\right]$

EPIC flux in a $0.2-10 \mathrm{keV}$ energy band. Determined from the sum of mos1-, mos2-, and pn-count rates in the $0.2-12.0 \mathrm{keV}$ via an energy conversion factor of $1.61452 \mathrm{E}-12\left[\mathrm{erg} \mathrm{cm}^{-2}\right]$.

Table A.1. X-ray source list.

\begin{tabular}{|c|c|c|c|c|c|c|c|c|}
\hline $\begin{array}{l}\text { (1) } \\
\text { ID }\end{array}$ & $\begin{array}{c}(2) \\
\text { Name } \\
\text { XMMU J... }\end{array}$ & $\begin{array}{c}(3) \\
\mathrm{CR} \\
{\left[\mathrm{ks}^{-1}\right]} \\
\end{array}$ & $\begin{array}{l}(4) \\
\text { ML }\end{array}$ & $\begin{array}{c}(5) \\
\sigma_{\mathrm{X}} \\
{[\operatorname{arcsec}]}\end{array}$ & $\begin{array}{c}(6) \\
H R 1\end{array}$ & $\begin{array}{c}(7) \\
H R 2\end{array}$ & $\begin{array}{c}(8) \\
H R 3\end{array}$ & $\begin{array}{c}9) \\
\text { Flux } \\
{\left[10^{-14} \mathrm{erg} \mathrm{s}^{-1} \mathrm{~cm}^{-2}\right]}\end{array}$ \\
\hline 1 & XMMU J031549.7-551810 & $101.0 \pm 2.1$ & 6918.8 & 0.72 & $0.195 \pm 0.033$ & $-0.617 \pm 0.032$ & $-0.524 \pm 0.081$ & 16.31 \\
\hline 2 & XMMU J031547.5-552902 & $296.3 \pm 6.4$ & 7886.9 & 0.72 & $0.316 \pm 0.034$ & $-0.681 \pm 0.030$ & $-0.362 \pm 0.087$ & 47.85 \\
\hline 3 & XMMU J031732.8-552025 & $181.3 \pm 8.7$ & 2130.2 & 0.77 & $0.788 \pm 0.066$ & $-0.587 \pm 0.083$ & $-0.381 \pm 0.198$ & 29.27 \\
\hline 4 & XMMU J031334.1-552642 & $232.7 \pm 6.3$ & 4202.8 & 0.74 & $-0.037 \pm 0.040$ & $-0.760 \pm 0.038$ & $-0.672 \pm 0.133$ & 37.56 \\
\hline 5 & XMMU J031650.3-551109 & $51.1 \pm 2.6$ & 1326.8 & 0.78 & $0.075 \pm 0.089$ & $-0.750 \pm 0.099$ & $-0.985 \pm 0.410$ & 8.244 \\
\hline 6 & XMMU J031605.9-551538 & $37.0 \pm 1.3$ & 1763.4 & 0.76 & $0.061 \pm 0.053$ & $-0.690 \pm 0.057$ & $-0.519 \pm 0.197$ & 5.966 \\
\hline 7 & XMMU J031505.7-550942 & $24.6 \pm 1.0$ & 1197.7 & 0.78 & $-0.095 \pm 0.061$ & $-0.606 \pm 0.073$ & $-0.864 \pm 0.200$ & 3.967 \\
\hline 8 & XMMU J031456.2-552006 & $24.6 \pm 1.0$ & 1025.1 & 0.78 & $0.760 \pm 0.070$ & $-0.186 \pm 0.074$ & $-0.287 \pm 0.098$ & 3.967 \\
\hline 9 & XMMU J031510.1-551313 & $21.3 \pm 0.9$ & 1151.4 & 0.77 & $0.564 \pm 0.056$ & $-0.554 \pm 0.056$ & $-0.520 \pm 0.133$ & 3.438 \\
\hline 10 & XMMU J031328.3-551019 & $60.3 \pm 2.2$ & 1627.3 & 0.77 & $0.570 \pm 0.051$ & $-0.541 \pm 0.051$ & $-0.281 \pm 0.105$ & 9.740 \\
\hline 11 & XMMU J031511.4-550927 & $24.7 \pm 1.0$ & 1123.8 & 0.77 & $0.206 \pm 0.061$ & $-0.664 \pm 0.060$ & $-0.616 \pm 0.187$ & 3.984 \\
\hline 12 & XMMU J031432.4-551440 & $21.1 \pm 0.9$ & 988.4 & 0.81 & $0.325 \pm 0.057$ & $-0.984 \pm 0.026$ & - & 3.405 \\
\hline 13 & XMMU J031351.1-551837 & $29.3 \pm 1.4$ & 796.3 & 0.81 & $0.041 \pm 0.074$ & $-0.702 \pm 0.078$ & $-0.189 \pm 0.233$ & 4.733 \\
\hline 14 & XMMU J031638.1-550635 & $33.6 \pm 1.8$ & 727.7 & 0.83 & $0.184 \pm 0.085$ & $-0.740 \pm 0.083$ & $-0.504 \pm 0.327$ & 5.419 \\
\hline 15 & XMMU J031552.8-550816 & $17.6 \pm 1.0$ & 563.1 & 0.82 & $0.526 \pm 0.087$ & $-0.552 \pm 0.091$ & $-0.363 \pm 0.200$ & 2.847 \\
\hline 16 & XMMU J031538.3-550140 & $41.4 \pm 2.0$ & 916.2 & 0.80 & $0.332 \pm 0.073$ & $-0.575 \pm 0.072$ & $-0.359 \pm 0.168$ & 6.678 \\
\hline 17 & XMMU J031528.6-551029 & $24.9 \pm 1.0$ & 1053.5 & 0.80 & $0.381 \pm 0.054$ & $-0.690 \pm 0.052$ & $-0.446 \pm 0.181$ & 4.022 \\
\hline 18 & XMMU J031421.3-552403 & $18.9 \pm 1.2$ & 403.9 & 0.87 & $0.454 \pm 0.093$ & $-0.669 \pm 0.091$ & $-0.614 \pm 0.344$ & 3.056 \\
\hline 19 & XMMU J031525.3-551827 & $13.2 \pm 0.8$ & 460.7 & 0.84 & $0.399 \pm 0.082$ & $-0.648 \pm 0.080$ & $-0.393 \pm 0.247$ & 2.129 \\
\hline 20 & XMMU J031621.6-551759 & $17.1 \pm 1.3$ & 380.8 & 0.87 & $0.569 \pm 0.119$ & $-0.432 \pm 0.117$ & $-0.507 \pm 0.253$ & 2.758 \\
\hline 21 & XMMU J031626.3-552250 & $19.3 \pm 1.5$ & 314.2 & 0.94 & $0.529 \pm 0.118$ & $-0.703 \pm 0.106$ & $-0.819 \pm 0.353$ & 3.110 \\
\hline 22 & XMMU J031519.9-550230 & $24.4 \pm 1.5$ & 473.6 & 0.89 & $0.238 \pm 0.101$ & $-0.540 \pm 0.107$ & $-0.128 \pm 0.223$ & 3.936 \\
\hline 23 & XMMU J031432.3-551959 & $15.5 \pm 1.0$ & 400.6 & 0.87 & $0.313 \pm 0.092$ & $-0.691 \pm 0.098$ & $-0.118 \pm 0.273$ & 2.499 \\
\hline 24 & XMMU J031703.7-550513 & $43.9 \pm 3.5$ & 339.3 & 0.97 & $0.326 \pm 0.128$ & $-0.480 \pm 0.123$ & $-0.669 \pm 0.228$ & 7.091 \\
\hline 25 & XMMU J031534.9-551925 & $14.7 \pm 1.0$ & 448.3 & 0.85 & $0.352 \pm 0.093$ & $-0.611 \pm 0.097$ & $-0.608 \pm 0.254$ & 2.373 \\
\hline 26 & XMMU J031436.1-551402 & $11.0 \pm 0.7$ & 326.8 & 0.90 & $0.340 \pm 0.101$ & $-0.732 \pm 0.109$ & $-0.275 \pm 0.390$ & 1.770 \\
\hline 27 & XMMU J031538.9-552219 & $13.1 \pm 1.0$ & 285.5 & 0.92 & $0.290 \pm 0.121$ & $-0.862 \pm 0.124$ & $-0.227 \pm 0.801$ & 2.116 \\
\hline 28 & XMMU J031507.7-550456 & $15.6 \pm 1.0$ & 404.5 & 0.89 & $0.166 \pm 0.088$ & $-0.702 \pm 0.094$ & $-0.711 \pm 0.349$ & 2.524 \\
\hline 29 & XMMU J031511.2-551530 & $11.0 \pm 0.7$ & 448.1 & 0.86 & $0.312 \pm 0.080$ & $-1.000 \pm 0.042$ & - & 1.780 \\
\hline
\end{tabular}


Table A.1. continued.

\begin{tabular}{|c|c|c|c|c|c|c|c|c|}
\hline $\begin{array}{l}\text { (1) } \\
\text { ID }\end{array}$ & $\begin{array}{c}(2) \\
\text { Name } \\
\text { XMMU J... }\end{array}$ & $\begin{array}{c}(3) \\
\mathrm{CR} \\
{\left[\mathrm{ks}^{-1}\right]}\end{array}$ & $\begin{array}{l}\text { (4) } \\
\text { ML }\end{array}$ & $\begin{array}{c}(5) \\
\sigma_{\mathrm{X}} \\
{[\operatorname{arcsec}]}\end{array}$ & $\begin{array}{c}(6) \\
H R 1\end{array}$ & $\begin{array}{c}(7) \\
H R 2\end{array}$ & $\begin{array}{c}(8) \\
H R 3\end{array}$ & $\begin{array}{c}\text { (9) } \\
\text { Flux } \\
{\left[10^{-14} \mathrm{erg} \mathrm{s}^{-1} \mathrm{~cm}^{-2}\right]}\end{array}$ \\
\hline 30 & XMMU J031659.7-551622 & $14.9 \pm 1.3$ & 207.6 & 0.99 & $1.000 \pm 0.095$ & $0.085 \pm 0.167$ & $-0.237 \pm 0.188$ & 2.408 \\
\hline 31 & XMMU J031706.8-551948 & $32.0 \pm 2.6$ & 297.5 & 0.97 & $0.650 \pm 0.135$ & $-0.251 \pm 0.133$ & $-0.551 \pm 0.190$ & 5.159 \\
\hline 32 & XMMU J031547.2-551755 & $12.5 \pm 0.9$ & 279.1 & 0.88 & $0.908 \pm 0.090$ & $-0.344 \pm 0.098$ & $-0.523 \pm 0.177$ & 2.018 \\
\hline 33 & XMMU J031319.5-551616 & $19.9 \pm 1.4$ & 295.1 & 0.93 & $0.531 \pm 0.118$ & $-0.551 \pm 0.122$ & $-0.566 \pm 0.277$ & 3.212 \\
\hline 34 & XMMU J031559.3-552637 & $28.2 \pm 1.9$ & 476.3 & 0.88 & $-0.240 \pm 0.095$ & $-0.668 \pm 0.122$ & $-0.675 \pm 0.413$ & 4.560 \\
\hline 35 & XMMU J031503.3-551906 & $9.4 \pm 0.7$ & 249.8 & 0.90 & $0.931 \pm 0.101$ & $-0.292 \pm 0.134$ & $0.094 \pm 0.166$ & 1.513 \\
\hline 36 & XMMU J031411.5-551830 & $8.5 \pm 0.8$ & 164.2 & 1.01 & $0.601 \pm 0.174$ & $-0.459 \pm 0.160$ & $-0.481 \pm 0.319$ & 1.365 \\
\hline 37 & XMMU J031236.3-545314 & $67.1 \pm 5.3$ & 256.9 & 1.04 & $0.233 \pm 0.126$ & $-0.513 \pm 0.123$ & $-0.542 \pm 0.270$ & 10.84 \\
\hline 38 & XMMU J031630.2-551909 & $21.6 \pm 1.4$ & 414.3 & 0.89 & $0.254 \pm 0.087$ & $-0.819 \pm 0.075$ & $-0.398 \pm 0.429$ & 3.481 \\
\hline 39 & XMMU J031339.7-550151 & $19.8 \pm 1.5$ & 249.8 & 1.00 & $0.769 \pm 0.122$ & $-0.402 \pm 0.122$ & $-0.338 \pm 0.209$ & 3.201 \\
\hline 40 & XMMU J031558.0-545549 & $30.9 \pm 3.1$ & 180.8 & 1.12 & $0.433 \pm 0.140$ & $-0.819 \pm 0.117$ & $-0.271 \pm 0.658$ & 4.982 \\
\hline 41 & XMMU J031608.1-551723 & $8.7 \pm 0.9$ & 151.6 & 1.01 & $0.194 \pm 0.128$ & $-0.947 \pm 0.100$ & - & 1.410 \\
\hline 42 & XMMU J031548.8-552245 & $12.3 \pm 1.0$ & 216.1 & 0.96 & $0.231 \pm 0.120$ & $-0.647 \pm 0.123$ & $-0.693 \pm 0.396$ & 1.992 \\
\hline 43 & XMMU J031332.4-551050 & $12.2 \pm 1.0$ & 179.3 & 1.03 & $0.549 \pm 0.102$ & $-0.933 \pm 0.085$ & $-1.000 \pm 0.982$ & 1.973 \\
\hline 44 & XMMU J031346.7-550031 & $14.1 \pm 1.4$ & 119.6 & 1.18 & $0.366 \pm 0.154$ & $-0.769 \pm 0.132$ & $0.364 \pm 0.325$ & 2.279 \\
\hline 45 & XMMU J031451.5-551932 & $5.3 \pm 0.6$ & 89.0 & 1.13 & $1.000 \pm 0.087$ & $-0.301 \pm 0.168$ & $-0.363 \pm 0.293$ & 0.853 \\
\hline 46 & XMMU J031607.4-552322 & $13.2 \pm 1.2$ & 183.7 & 1.07 & $0.052 \pm 0.155$ & $-0.582 \pm 0.174$ & $-0.514 \pm 0.479$ & 2.131 \\
\hline 47 & XMMU J031538.8-551043 & $4.5 \pm 0.5$ & 82.4 & 1.12 & $0.925 \pm 0.124$ & $-0.407 \pm 0.158$ & $-0.886 \pm 0.260$ & 0.731 \\
\hline 48 & XMMU J031458.5-545732 & $21.7 \pm 1.9$ & 183.1 & 1.09 & $0.551 \pm 0.126$ & $-0.925 \pm 0.093$ & - & 3.502 \\
\hline 49 & XMMU J031638.4-552013 & $13.5 \pm 1.2$ & 167.9 & 1.03 & $1.000 \pm 0.050$ & $-0.413 \pm 0.134$ & $-0.430 \pm 0.248$ & 2.172 \\
\hline 50 & XMMU J031410.1-551746 & $7.6 \pm 0.7$ & 112.1 & 1.11 & $0.969 \pm 0.087$ & $-0.367 \pm 0.157$ & $-0.117 \pm 0.245$ & 1.233 \\
\hline 51 & XMMU J031630.6-551501 & $10.4 \pm 1.0$ & 154.6 & 1.05 & $0.617 \pm 0.145$ & $-0.411 \pm 0.147$ & $-0.530 \pm 0.334$ & 1.687 \\
\hline 52 & XMMU J031451.7-545713 & $15.6 \pm 2.0$ & 89.9 & 1.26 & $0.033 \pm 0.209$ & $-0.662 \pm 0.231$ & - & 2.518 \\
\hline 53 & XMMU J031358.9-551754 & $6.7 \pm 0.8$ & 75.4 & 1.20 & $0.574 \pm 0.185$ & $-0.478 \pm 0.179$ & $-1.000 \pm 0.487$ & 1.078 \\
\hline 54 & XMMU J031612.7-545910 & $11.3 \pm 1.5$ & 68.3 & 1.34 & $0.175 \pm 0.222$ & $-0.852 \pm 0.197$ & $0.242 \pm 0.888$ & 1.820 \\
\hline 55 & XMMU J031457.3-552027 & $4.6 \pm 0.6$ & 45.0 & 1.31 & $0.421 \pm 0.203$ & $-0.759 \pm 0.256$ & $-0.219 \pm 0.899$ & 0.741 \\
\hline 56 & XMMU J031250.9-551728 & $28.2 \pm 2.6$ & 208.6 & 1.07 & $0.142 \pm 0.172$ & $-0.208 \pm 0.174$ & $-0.940 \pm 0.192$ & 4.559 \\
\hline 57 & XMMU J031344.2-551922 & $8.2 \pm 0.9$ & 74.9 & 1.22 & $0.349 \pm 0.179$ & $-0.547 \pm 0.168$ & $-0.259 \pm 0.358$ & 1.329 \\
\hline 58 & XMMU J031301.8-552224 & $21.0 \pm 2.1$ & 103.4 & 1.20 & $0.128 \pm 0.157$ & $-0.412 \pm 0.170$ & $-0.862 \pm 0.253$ & 3.389 \\
\hline 59 & XMMU J031339.6-551426 & $10.9 \pm 0.9$ & 138.7 & 1.06 & $0.126 \pm 0.120$ & $-0.640 \pm 0.135$ & $0.252 \pm 0.253$ & 1.753 \\
\hline 60 & XMMU J031426.4-551746 & $6.5 \pm 0.6$ & 97.6 & 1.14 & $0.211 \pm 0.155$ & $-0.468 \pm 0.165$ & $-0.493 \pm 0.336$ & 1.042 \\
\hline 61 & XMMU J031238.7-550232 & $2.9 \pm 1.1$ & 9.6 & 2.26 & $1.000 \pm 0.892$ & $-1.000 \pm 0.643$ & - & 0.465 \\
\hline 62 & XMMU J031442.0-545555 & $22.3 \pm 3.0$ & 82.8 & 1.35 & $0.625 \pm 0.149$ & $-0.795 \pm 0.128$ & $-0.043 \pm 0.495$ & 3.595 \\
\hline 63 & XMMU J031517.1-550602 & $7.2 \pm 0.7$ & 130.2 & 1.07 & $0.651 \pm 0.143$ & $-0.398 \pm 0.132$ & $-1.000 \pm 0.197$ & 1.164 \\
\hline 64 & XMMU J031334.0-551021 & $7.3 \pm 0.9$ & 44.9 & 1.43 & $0.240 \pm 0.187$ & $-0.620 \pm 0.182$ & $0.128 \pm 0.361$ & 1.179 \\
\hline 65 & XMMU J031402.2-552756 & $2.2 \pm 1.3$ & 9.9 & 2.07 & - & - & - & 0.360 \\
\hline 66 & XMMU J031500.8-550718 & $4.0 \pm 0.6$ & 51.4 & 1.24 & $0.720 \pm 0.184$ & $-0.585 \pm 0.167$ & $-0.458 \pm 0.488$ & 0.652 \\
\hline 67 & XMMU J031310.6-551312 & $12.0 \pm 1.1$ & 109.4 & 1.09 & $0.232 \pm 0.153$ & $-0.609 \pm 0.154$ & $0.062 \pm 0.323$ & 1.945 \\
\hline 68 & XMMU J031338.2-552303 & $12.1 \pm 1.2$ & 107.1 & 1.13 & $0.105 \pm 0.181$ & $-0.569 \pm 0.200$ & $-0.183 \pm 0.442$ & 1.954 \\
\hline 69 & XMMU J031415.4-551910 & $3.1 \pm 0.6$ & 19.3 & 1.54 & $0.639 \pm 0.293$ & $-0.867 \pm 0.260$ & $0.676 \pm 0.595$ & 0.500 \\
\hline 70 & XMMU J031620.8-550648 & $2.9 \pm 1.0$ & 13.4 & 1.84 & $-0.259 \pm 0.652$ & - & $0.959 \pm 0.482$ & 0.464 \\
\hline 71 & XMMU J031714.1-550131 & $17.9 \pm 2.5$ & 61.4 & 1.45 & $0.643 \pm 0.182$ & $-0.856 \pm 0.146$ & $-0.304 \pm 0.861$ & 2.892 \\
\hline 72 & XMMU J031660.0-545855 & $4.9 \pm 2.1$ & 9.5 & 2.38 & $-0.145 \pm 0.860$ & $-1.000 \pm 0.872$ & - & 0.787 \\
\hline 73 & XMMU J031321.3-552046 & $12.9 \pm 1.5$ & 87.6 & 1.24 & $0.379 \pm 0.155$ & $-0.806 \pm 0.133$ & $0.399 \pm 0.371$ & 2.086 \\
\hline 75 & XMMU J031601.3-550516 & $5.6 \pm 0.7$ & 56.6 & 1.25 & $0.391 \pm 0.195$ & $-0.521 \pm 0.200$ & $-0.874 \pm 0.360$ & 0.909 \\
\hline 76 & XMMU J031549.7-550908 & $4.9 \pm 0.6$ & 67.5 & 1.28 & $0.472 \pm 0.179$ & $-0.819 \pm 0.170$ & $0.177 \pm 0.655$ & 0.798 \\
\hline 78 & XMMU J031502.2-552611 & $4.6 \pm 1.1$ & 19.0 & 1.50 & $1.000 \pm 0.332$ & $-0.561 \pm 0.316$ & $0.263 \pm 0.505$ & 0.748 \\
\hline 79 & XMMU J031343.7-552007 & $1.8 \pm 0.6$ & 5.4 & 1.98 & - & $0.479 \pm 0.739$ & $-0.832 \pm 0.777$ & 0.295 \\
\hline 80 & XMMU J031359.8-545716 & $11.7 \pm 1.8$ & 52.1 & 1.45 & $0.156 \pm 0.257$ & $-0.825 \pm 0.253$ & - & 1.886 \\
\hline 81 & XMMU J031343.7-550153 & $12.7 \pm 1.3$ & 98.6 & 1.20 & $0.510 \pm 0.136$ & $-0.625 \pm 0.132$ & $-0.931 \pm 0.224$ & 2.046 \\
\hline 83 & XMMU J031448.2-552231 & $6.5 \pm 0.8$ & 87.9 & 1.16 & $0.417 \pm 0.142$ & $-0.737 \pm 0.148$ & $-0.682 \pm 0.632$ & 1.046 \\
\hline 84 & XMMU J031621.8-552038 & $5.8 \pm 1.1$ & 42.0 & 1.41 & $0.979 \pm 0.154$ & $-0.739 \pm 0.275$ & $-0.087 \pm 0.845$ & 0.934 \\
\hline 85 & XMMU J031523.1-545919 & $1.4 \pm 0.7$ & 6.4 & 2.28 & $-1.000 \pm 0.502$ & - & - & 0.222 \\
\hline 86 & XMMU J031345.0-551612 & $5.6 \pm 0.7$ & 49.8 & 1.32 & $0.558 \pm 0.194$ & $-0.403 \pm 0.193$ & $-1.000 \pm 0.389$ & 0.911 \\
\hline
\end{tabular}


Table A.1. continued.

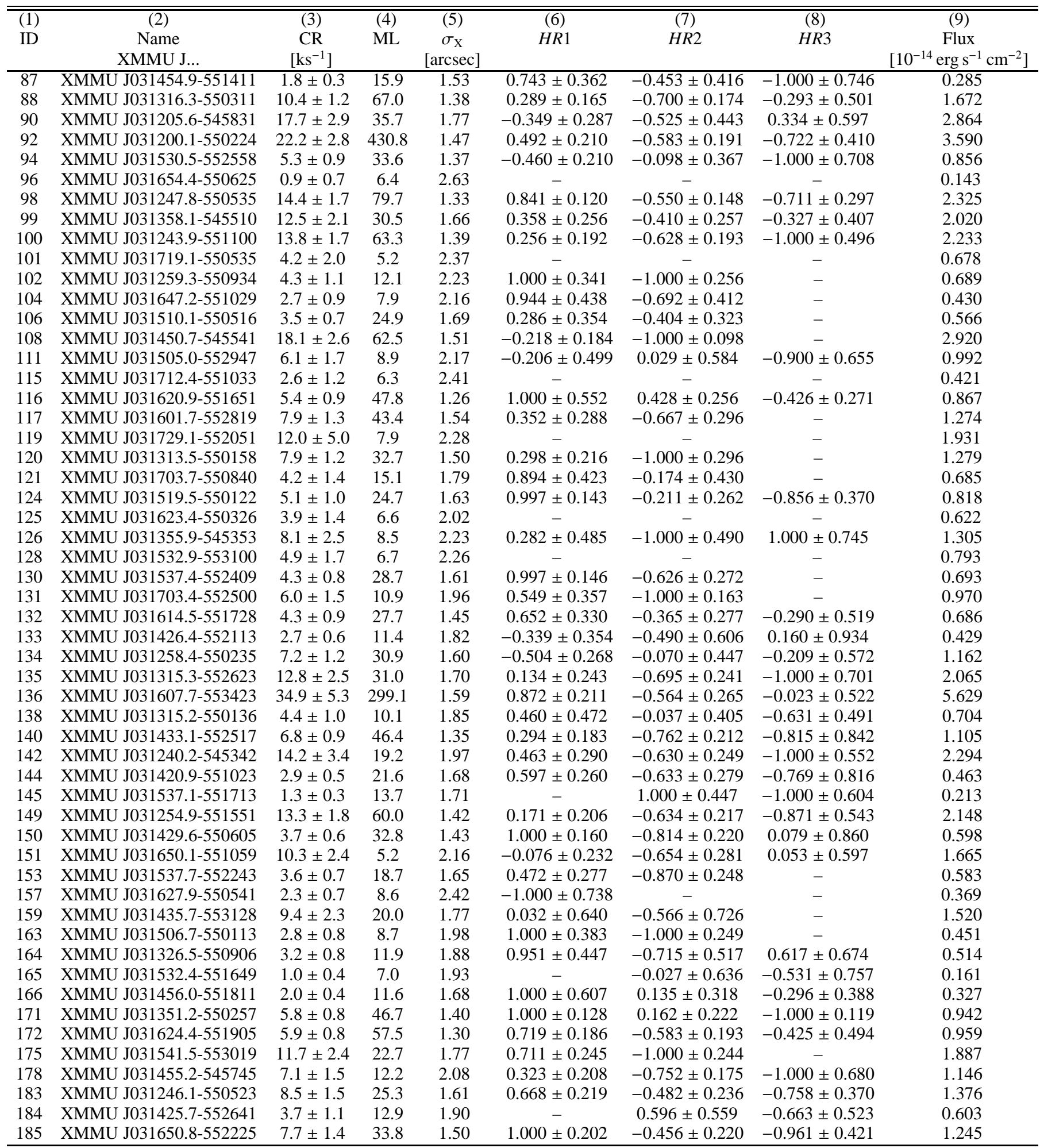


Table A.1. continued.

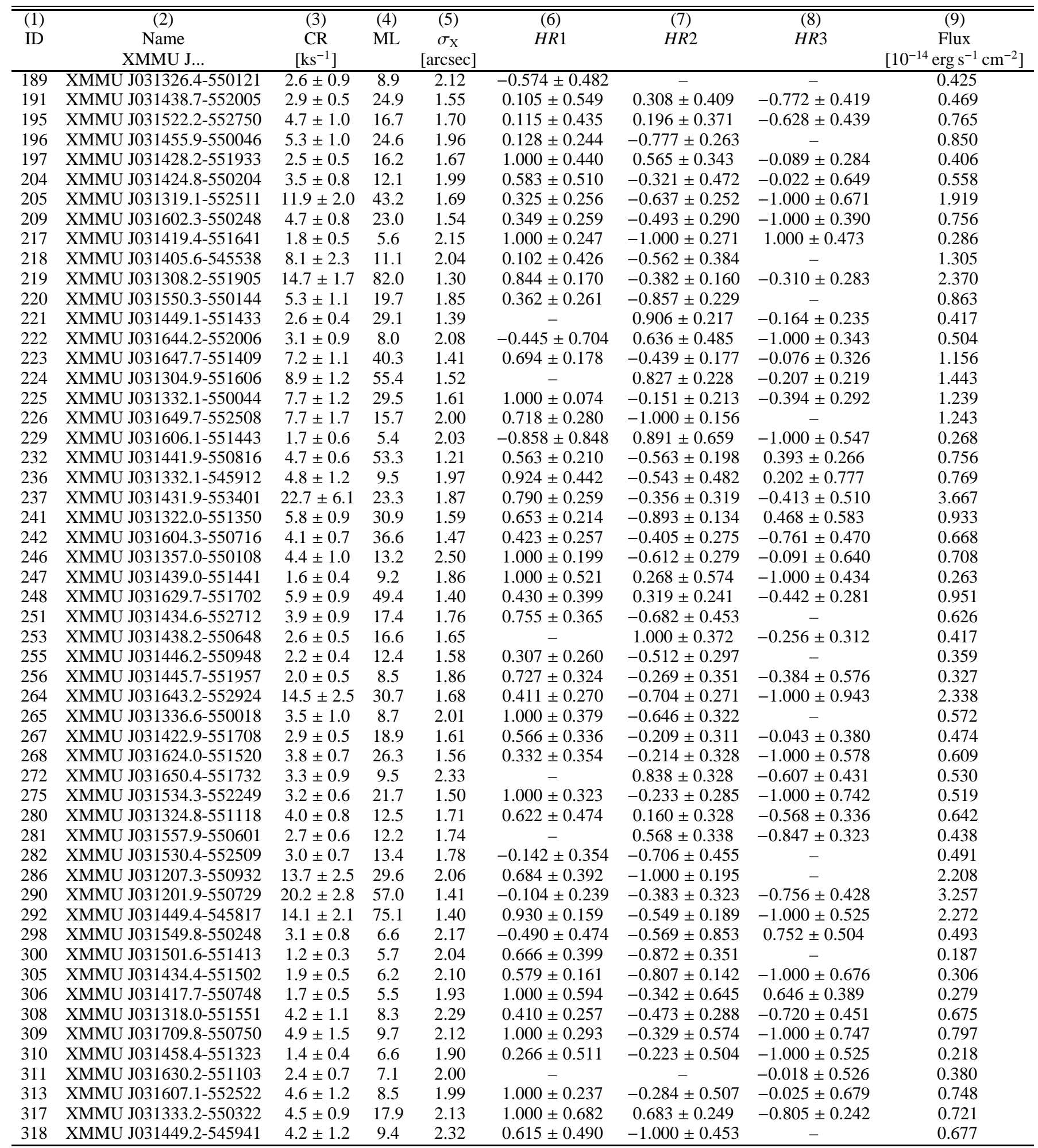


Table A.1. continued.

\begin{tabular}{|c|c|c|c|c|c|c|c|c|}
\hline $\begin{array}{l}\text { (1) } \\
\text { ID }\end{array}$ & $\begin{array}{c}(2) \\
\text { Name } \\
\text { XMMU J... }\end{array}$ & $\begin{array}{c}(3) \\
\mathrm{CR} \\
{\left[\mathrm{ks}^{-1}\right]}\end{array}$ & $\begin{array}{l}\text { (4) } \\
\text { ML }\end{array}$ & $\begin{array}{c}(5) \\
\sigma_{\mathrm{X}} \\
{[\operatorname{arcsec}]}\end{array}$ & $\begin{array}{c}(6) \\
H R 1\end{array}$ & $\begin{array}{c}(7) \\
H R 2\end{array}$ & $\begin{array}{c}(8) \\
H R 3\end{array}$ & $\begin{array}{c}\text { (9) } \\
\text { Flux } \\
{\left[10^{-14} \mathrm{erg} \mathrm{s}^{-1} \mathrm{~cm}^{-2}\right]}\end{array}$ \\
\hline 321 & XMMU J031422.9-550842 & $1.7 \pm 0.4$ & 7.4 & 2.03 & $0.406 \pm 0.439$ & $-1.000 \pm 0.191$ & - & 0.272 \\
\hline 325 & XMMU J031443.4-552029 & $3.6 \pm 0.7$ & 16.3 & 1.59 & $0.498 \pm 0.269$ & $-0.346 \pm 0.266$ & $0.085 \pm 0.360$ & 0.574 \\
\hline 332 & XMMU J031402.0-552720 & $7.1 \pm 1.5$ & 17.0 & 1.80 & $0.332 \pm 0.266$ & $-0.665 \pm 0.279$ & $-1.000 \pm 0.706$ & 1.139 \\
\hline 334 & XMMU J031631.7-551228 & $6.4 \pm 1.0$ & 73.4 & 1.29 & $0.844 \pm 0.154$ & $-0.761 \pm 0.141$ & $-0.262 \pm 0.654$ & 1.032 \\
\hline 335 & XMMU J031357.2-550432 & $2.6 \pm 0.7$ & 7.7 & 1.95 & $1.000 \pm 0.421$ & $-0.147 \pm 0.350$ & $-1.000 \pm 0.677$ & 0.415 \\
\hline 338 & XMMU J031227.1-551221 & $10.2 \pm 2.4$ & 13.3 & 1.91 & $0.040 \pm 0.380$ & $-0.290 \pm 0.406$ & $-1.000 \pm 0.586$ & 1.647 \\
\hline 339 & XMMU J031432.3-552927 & $6.0 \pm 1.4$ & 9.8 & 2.42 & $-0.321 \pm 0.359$ & $0.271 \pm 0.385$ & $-1.000 \pm 0.665$ & 0.964 \\
\hline 345 & XMMU J031316.7-552239 & $13.3 \pm 1.7$ & 63.6 & 1.33 & $0.103 \pm 0.172$ & $-0.457 \pm 0.192$ & $-1.000 \pm 0.632$ & 2.153 \\
\hline 346 & XMMU J031633.5-550412 & $2.9 \pm 0.8$ & 17.0 & 2.13 & - & - & - & 0.462 \\
\hline 349 & XMMU J031541.0-552227 & $4.1 \pm 0.9$ & 14.9 & 1.75 & $0.635 \pm 0.231$ & $-0.540 \pm 0.210$ & $0.145 \pm 0.352$ & 0.655 \\
\hline 355 & XMMU J031517.8-545953 & $6.1 \pm 1.3$ & 13.2 & 2.13 & $0.156 \pm 0.463$ & $0.340 \pm 0.317$ & $-0.382 \pm 0.340$ & 0.979 \\
\hline 356 & XMMU J031519.6-552333 & $2.2 \pm 0.7$ & 5.7 & 2.06 & $-1.000 \pm 0.523$ & - & $-0.305 \pm 0.894$ & 0.349 \\
\hline 360 & XMMU J031312.0-551718 & $5.5 \pm 1.3$ & 15.6 & 1.79 & $1.000 \pm 0.931$ & $-0.096 \pm 0.459$ & $-0.207 \pm 0.585$ & 0.881 \\
\hline 361 & XMMU J031314.6-550320 & $5.5 \pm 1.1$ & 12.4 & 1.93 & $0.254 \pm 0.427$ & $0.069 \pm 0.362$ & $-0.210 \pm 0.372$ & 0.882 \\
\hline 364 & XMMU J031412.3-552555 & $6.7 \pm 1.0$ & 29.9 & 1.64 & $0.379 \pm 0.196$ & $-0.611 \pm 0.204$ & $-1.000 \pm 0.530$ & 1.077 \\
\hline 365 & XMMU J031526.1-551937 & $2.5 \pm 0.6$ & 7.3 & 2.37 & $0.746 \pm 0.381$ & $-0.052 \pm 0.345$ & $-0.068 \pm 0.417$ & 0.401 \\
\hline 367 & XMMU J031635.1-550352 & $11.4 \pm 2.2$ & 50.7 & 1.87 & $0.825 \pm 0.226$ & $-0.455 \pm 0.243$ & $-0.263 \pm 0.430$ & 1.848 \\
\hline 371 & XMMU J031620.5-550330 & $3.5 \pm 0.9$ & 13.1 & 1.94 & - & $1.000 \pm 0.734$ & $0.341 \pm 0.401$ & 0.561 \\
\hline 373 & XMMU J031502.6-550508 & $2.3 \pm 0.6$ & 6.5 & 2.00 & $-0.264 \pm 0.417$ & $0.061 \pm 0.488$ & $-0.237 \pm 0.555$ & 0.373 \\
\hline 375 & XMMU J031447.2-553258 & $15.2 \pm 3.3$ & 19.6 & 2.06 & $0.602 \pm 0.376$ & $-1.000 \pm 0.515$ & $1.000 \pm 0.575$ & 2.459 \\
\hline 376 & XMMU J031717.8-551948 & $5.1 \pm 1.5$ & 12.9 & 1.96 & $0.659 \pm 0.487$ & $-0.229 \pm 0.534$ & - & 0.819 \\
\hline 377 & XMMU J031653.3-550838 & $4.6 \pm 1.1$ & 18.4 & 1.74 & $0.479 \pm 0.358$ & $-0.525 \pm 0.361$ & $-0.442 \pm 0.755$ & 0.743 \\
\hline 380 & XMMU J031449.7-552336 & $2.1 \pm 0.7$ & 5.0 & 2.24 & $1.000 \pm 0.312$ & $-1.000 \pm 0.486$ & - & 0.335 \\
\hline 381 & XMMU J031644.6-552707 & $25.5 \pm 2.8$ & 162.7 & 1.15 & $0.386 \pm 0.159$ & $-0.698 \pm 0.158$ & $0.220 \pm 0.358$ & 4.112 \\
\hline 382 & XMMU J031247.4-551653 & $8.9 \pm 1.8$ & 28.2 & 1.89 & $0.848 \pm 0.270$ & $-0.927 \pm 0.167$ & - & 1.445 \\
\hline 385 & XMMU J031318.2-550537 & $5.6 \pm 1.0$ & 24.9 & 1.57 & $0.844 \pm 0.364$ & $0.346 \pm 0.239$ & $-0.369 \pm 0.247$ & 0.910 \\
\hline 388 & XMMU J031401.2-545957 & $100.7 \pm 5.8$ & 775.3 & 0.71 & $0.128 \pm 0.063$ & $-0.652 \pm 0.060$ & $-0.284 \pm 0.162$ & 16.26 \\
\hline 389 & XMMU J031539.0-550336 & $2.5 \pm 0.7$ & 7.3 & 1.96 & $0.710 \pm 0.326$ & $-0.352 \pm 0.349$ & $-0.904 \pm 0.570$ & 0.399 \\
\hline 397 & XMMU J031638.3-545924 & $15.7 \pm 2.5$ & 57.8 & 1.50 & $0.560 \pm 0.235$ & $-0.506 \pm 0.228$ & $-0.603 \pm 0.441$ & 2.529 \\
\hline 403 & XMMU J031225.8-550554 & $18.6 \pm 2.2$ & 67.9 & 1.41 & $0.151 \pm 0.182$ & $-0.448 \pm 0.187$ & $-0.730 \pm 0.309$ & 3.005 \\
\hline 408 & XMMU J031554.2-551004 & $2.3 \pm 0.5$ & 7.9 & 1.85 & $1.000 \pm 0.214$ & $-0.051 \pm 0.327$ & $-0.129 \pm 0.376$ & 0.365 \\
\hline 410 & XMMU J031502.0-552742 & $4.1 \pm 1.1$ & 5.8 & 2.17 & $0.146 \pm 0.623$ & $-0.383 \pm 0.684$ & $0.204 \pm 0.830$ & 0.662 \\
\hline 412 & XMMU J031218.4-550648 & $7.0 \pm 1.9$ & 6.9 & 2.72 & $0.623 \pm 0.300$ & $-1.000 \pm 0.137$ & - & 1.134 \\
\hline 413 & XMMU J031723.0-550222 & $9.5 \pm 2.4$ & 14.0 & 2.20 & $0.187 \pm 0.796$ & $0.502 \pm 0.448$ & $-0.799 \pm 0.401$ & 1.526 \\
\hline 421 & XMMU J031431.3-550943 & $1.9 \pm 0.4$ & 10.1 & 2.21 & $0.842 \pm 0.241$ & $-1.000 \pm 0.238$ & - & 0.304 \\
\hline 422 & XMMU J031531.6-551049 & $2.3 \pm 0.4$ & 14.5 & 1.80 & $0.520 \pm 0.452$ & $0.229 \pm 0.283$ & $-0.923 \pm 0.295$ & 0.371 \\
\hline 431 & XMMU J031540.9-545649 & $6.6 \pm 1.3$ & 16.1 & 1.84 & $0.706 \pm 0.284$ & $-0.531 \pm 0.254$ & $-1.000 \pm 0.426$ & 1.060 \\
\hline 433 & XMMU J031436.4-552739 & $3.7 \pm 1.0$ & 7.6 & 2.33 & - & $0.933 \pm 0.255$ & $-0.762 \pm 0.330$ & 0.595 \\
\hline 437 & XMMU J031534.0-550259 & $3.6 \pm 0.7$ & 14.9 & 1.64 & $0.645 \pm 0.221$ & $-0.609 \pm 0.236$ & $-1.000 \pm 0.440$ & 0.582 \\
\hline 438 & XMMU J031521.8-552338 & $2.1 \pm 0.6$ & 6.2 & 2.22 & $0.223 \pm 0.412$ & $-1.000 \pm 0.181$ & $1.000 \pm 0.318$ & 0.340 \\
\hline 444 & XMMU J031318.1-550600 & $3.9 \pm 0.9$ & 11.5 & 1.95 & $1.000 \pm 0.999$ & $0.617 \pm 0.307$ & $-0.358 \pm 0.276$ & 0.628 \\
\hline 447 & XMMU J031448.7-550037 & $3.6 \pm 1.0$ & 6.4 & 2.71 & $0.641 \pm 0.401$ & $-0.355 \pm 0.362$ & $-1.000 \pm 0.732$ & 0.587 \\
\hline 449 & XMMU J031543.3-550742 & $2.2 \pm 0.6$ & 6.4 & 2.40 & $-0.022 \pm 0.395$ & $-0.065 \pm 0.412$ & $-1.000 \pm 0.655$ & 0.357 \\
\hline 452 & XMMU J031618.2-545649 & $11.0 \pm 2.2$ & 23.9 & 1.77 & $0.545 \pm 0.220$ & $-0.595 \pm 0.205$ & $-0.921 \pm 0.578$ & 1.781 \\
\hline 453 & XMMU J031323.6-550445 & $3.9 \pm 1.0$ & 5.2 & 2.46 & $1.000 \pm 0.160$ & $0.019 \pm 0.339$ & $-0.773 \pm 0.395$ & 0.635 \\
\hline 456 & XMMU J031632.5-552743 & $7.5 \pm 1.7$ & 15.3 & 1.85 & $0.464 \pm 0.272$ & $-1.000 \pm 0.100$ & $1.000 \pm 0.257$ & 1.218 \\
\hline 460 & XMMU J031342.7-550700 & $5.4 \pm 0.8$ & 36.6 & 1.44 & $0.410 \pm 0.190$ & $-0.982 \pm 0.124$ & - & 0.872 \\
\hline 461 & XMMU J031714.5-551252 & $4.2 \pm 1.6$ & 6.1 & 2.29 & - & $1.000 \pm 0.285$ & $-0.407 \pm 0.440$ & 0.675 \\
\hline 462 & XMMU J031506.2-551626 & $1.9 \pm 0.4$ & 9.2 & 1.79 & $1.000 \pm 0.587$ & $0.165 \pm 0.269$ & $-0.384 \pm 0.343$ & 0.301 \\
\hline 463 & XMMU J031625.3-550839 & $3.1 \pm 0.8$ & 18.8 & 1.61 & $0.979 \pm 0.305$ & $-0.592 \pm 0.441$ & - & 0.506 \\
\hline 464 & XMMU J031250.3-550430 & $4.9 \pm 1.2$ & 11.4 & 2.05 & $0.083 \pm 0.378$ & $-1.000 \pm 0.865$ & - & 0.791 \\
\hline 468 & XMMU J031253.7-551005 & $9.3 \pm 1.4$ & 82.7 & 1.29 & $-0.984 \pm 0.057$ & - & - & 1.506 \\
\hline 470 & XMMU J031521.4-552123 & $3.0 \pm 0.6$ & 15.0 & 1.69 & $1.000 \pm 0.186$ & $-0.038 \pm 0.259$ & $-0.675 \pm 0.414$ & 0.477 \\
\hline 473 & XMMU J031532.5-551116 & $1.3 \pm 0.4$ & 5.3 & 3.35 & $1.000 \pm 0.523$ & $0.039 \pm 0.347$ & $-1.000 \pm 0.163$ & 0.216 \\
\hline
\end{tabular}


Table A.1. continued.

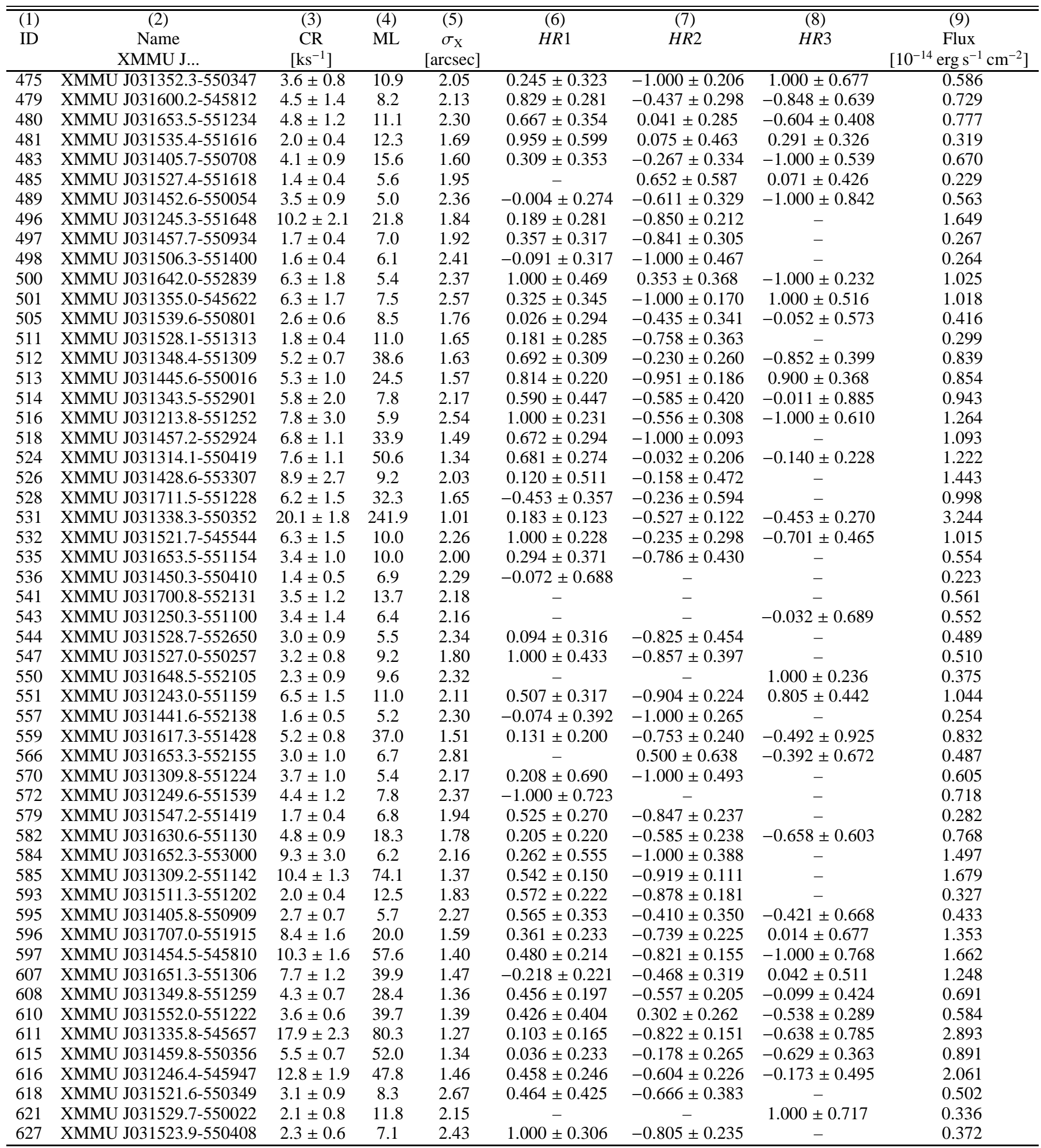


M. Krumpe et al.: The XMM-Newton survey in the Marano field. I., Online Material $p 8$

Table A.1. continued.

\begin{tabular}{|c|c|c|c|c|c|c|c|c|}
\hline$\overline{(1)}$ & $\overline{(2)}$ & $\overline{(3)}$ & $\overline{(4)}$ & $\overline{(5)}$ & $\overline{(6)}$ & $\overline{(7)}$ & $\overline{(8)}$ & $\overline{(9)}$ \\
\hline ID & $\begin{array}{c}\text { Name } \\
\text { XMMU J... }\end{array}$ & $\begin{array}{c}\mathrm{CR} \\
{\left[\mathrm{ks}^{-1}\right]}\end{array}$ & ML & $\begin{array}{c}\sigma_{\mathrm{X}} \\
{[\operatorname{arcsec}]}\end{array}$ & $H R 1$ & $H R 2$ & $H R 3$ & $\begin{array}{c}\text { Flux } \\
{\left[10^{-14} \mathrm{erg} \mathrm{s}^{-1} \mathrm{~cm}^{-2}\right]}\end{array}$ \\
\hline 630 & XMMU J031625.0-551122 & $1.5 \pm 0.8$ & 9.1 & 2.18 & $0.619 \pm 0.472$ & $-1.000 \pm 0.292$ & - & 0.239 \\
\hline 631 & XMMU J031532.8-551159 & $2.0 \pm 0.4$ & 7.5 & 1.72 & $0.673 \pm 0.320$ & $-0.634 \pm 0.308$ & $0.328 \pm 0.504$ & 0.323 \\
\hline 632 & XMMU J031624.5-552135 & $1.2 \pm 0.6$ & 5.5 & 2.25 & - & - & - & 0.193 \\
\hline 633 & XMMU J031532.5-545909 & $4.0 \pm 1.1$ & 8.0 & 2.01 & $-0.788 \pm 0.346$ & $0.437 \pm 0.829$ & $0.131 \pm 0.699$ & 0.641 \\
\hline 652 & XMMU J031530.3-550437 & $14.0 \pm 1.0$ & 328.4 & 0.89 & $0.350 \pm 0.106$ & $-0.765 \pm 0.096$ & $0.269 \pm 0.262$ & 2.262 \\
\hline 653 & XMMU J031647.5-551229 & $6.9 \pm 1.1$ & 46.2 & 1.31 & $0.397 \pm 0.212$ & $-0.615 \pm 0.227$ & $-0.298 \pm 0.616$ & 1.119 \\
\hline 654 & XMMU J031706.1-552716 & $34.8 \pm 3.6$ & 150.3 & 1.18 & $0.649 \pm 0.137$ & $-0.632 \pm 0.138$ & $-0.098 \pm 0.344$ & 5.627 \\
\hline 656 & XMMU J031326.1-550427 & $35.3 \pm 3.1$ & 320.8 & 0.98 & $1.000 \pm 0.033$ & $-0.183 \pm 0.096$ & $-0.470 \pm 0.135$ & 5.695 \\
\hline 660 & XMMU J031537.2-545947 & $3.5 \pm 1.0$ & 5.1 & 2.59 & $0.730 \pm 0.448$ & $-0.080 \pm 0.414$ & $-1.000 \pm 0.320$ & 0.568 \\
\hline 661 & XMMU J031706.8-552435 & $7.9 \pm 2.3$ & 12.7 & 2.53 & $0.805 \pm 0.490$ & $-1.000 \pm 0.568$ & $1.000 \pm 0.494$ & 1.275 \\
\hline 664 & XMMU J031611.1-552117 & $2.7 \pm 0.7$ & 6.2 & 2.23 & $0.249 \pm 0.330$ & $-0.631 \pm 0.380$ & - & 0.444 \\
\hline 665 & XMMU J031645.7-545900 & $5.9 \pm 1.8$ & 7.5 & 2.44 & $0.728 \pm 0.354$ & $-0.909 \pm 0.316$ & - & 0.958 \\
\hline 667 & XMMU J031348.0-551205 & $5.4 \pm 1.0$ & 25.1 & 2.79 & $-0.244 \pm 0.758$ & $-1.000 \pm 0.913$ & - & 0.875 \\
\hline 673 & XMMU J031540.4-551221 & $11.0 \pm 0.8$ & 334.6 & 0.88 & $0.788 \pm 0.095$ & $-0.534 \pm 0.103$ & $-0.068 \pm 0.204$ & 1.777 \\
\hline 675 & XMMU J031624.6-551143 & $9.8 \pm 1.0$ & 122.8 & 1.07 & $0.378 \pm 0.123$ & $-0.933 \pm 0.087$ & - & 1.589 \\
\hline 676 & XMMU J031641.5-552408 & $4.1 \pm 1.1$ & 6.1 & 2.08 & $1.000 \pm 0.178$ & $-0.218 \pm 0.357$ & $-0.124 \pm 0.481$ & 0.656 \\
\hline 680 & XMMU J031647.6-552142 & $2.3 \pm 0.9$ & 9.9 & 1.96 & $0.953 \pm 0.891$ & $-1.000 \pm 0.441$ & $1.000 \pm 0.510$ & 0.375 \\
\hline 681 & XMMU J031248.9-550705 & $1.6 \pm 0.8$ & 5.6 & 2.64 & $0.819 \pm 0.903$ & - & - & 0.265 \\
\hline 686 & XMMU J031346.7-551148 & $94.0 \pm 2.2$ & 4192.0 & 0.73 & $0.287 \pm 0.035$ & $-0.633 \pm 0.033$ & $-0.448 \pm 0.089$ & 15.18 \\
\hline 687 & XMMU J031302.5-552604 & $16.3 \pm 3.1$ & 26.4 & 1.88 & $0.252 \pm 0.226$ & $-0.450 \pm 0.237$ & $-0.952 \pm 0.296$ & 2.626 \\
\hline 688 & XMMU J031145.8-550653 & $189.3 \pm 11.1$ & 775.6 & 0.94 & $0.293 \pm 0.089$ & $-0.587 \pm 0.086$ & $-0.326 \pm 0.189$ & 30.56 \\
\hline 689 & XMMU J031140.1-550613 & $29.5 \pm 4.0$ & 50.3 & 1.84 & $0.094 \pm 0.186$ & $-0.723 \pm 0.174$ & - & 4.761 \\
\hline 690 & XMMU J031135.6-550314 & $57.4 \pm 9.1$ & 86.2 & 1.80 & $0.270 \pm 0.132$ & $-0.582 \pm 0.135$ & $-0.591 \pm 0.326$ & 9.267 \\
\hline 691 & XMMU J031134.5-550608 & $28.0 \pm 4.3$ & 46.9 & 1.64 & $0.579 \pm 0.178$ & $-0.794 \pm 0.141$ & $-0.462 \pm 0.592$ & 4.524 \\
\hline 692 & XMMU J031136.3-550514 & $36.3 \pm 4.7$ & 73.0 & 1.49 & $-0.005 \pm 0.198$ & $-0.439 \pm 0.227$ & $-0.287 \pm 0.405$ & 5.855 \\
\hline 693 & XMMU J031145.9-550702 & $336.5 \pm 20.0$ & 418.0 & 0.98 & $0.191 \pm 0.201$ & $-0.586 \pm 0.205$ & $-0.458 \pm 0.476$ & 54.32 \\
\hline 694 & XMMU J031146.4-550711 & $97.3 \pm 10.6$ & 53.0 & 1.24 & $0.283 \pm 0.048$ & $-0.609 \pm 0.046$ & $-0.411 \pm 0.109$ & 15.71 \\
\hline 695 & XMMU J031259.1-552556 & $45.5 \pm 6.3$ & 91.5 & 1.83 & $0.307 \pm 0.139$ & $-0.699 \pm 0.130$ & $0.106 \pm 0.330$ & 7.347 \\
\hline 696 & XMMU J031152.2-551049 & $28.0 \pm 4.7$ & 41.8 & 1.84 & $0.348 \pm 0.193$ & $-0.918 \pm 0.147$ & - & 4.527 \\
\hline
\end{tabular}




\section{Appendix B: Marginal X-ray source classification}

In this section we include spectroscopic classifications that are possible counterparts to X-ray sources with a detection likelihood $M L<5.0$. We list the optical and the X-ray properties of these sources. The objects given here are included in the optical and X-ray image section (Appendix C).

\section{B.1. Optical data table}

Table B.1. Optical properties of candidate counterparts of marginal Marano Field XMM-Newton X-ray sources. For a detailed explanation of the individual columns see Sect. 3.

\begin{tabular}{|c|c|c|c|c|c|c|c|c|c|c|c|c|c|c|}
\hline (1) & (2) & (3) & (4) & (5) & (6) & (7) & (8) & (9) & (10) & (11) & (12) & (13) & (14) & $(15)$ \\
\hline No & RA & Dec & Dist $_{\text {OX }}$ & $K$ & $R$ & Class & $z$ & Flags & $\log \left(L_{\mathrm{X}_{\mathrm{OBS}}}\right)$ & $M_{\mathrm{B}}$ & $\alpha_{\mathrm{OX}}$ & $N_{\mathrm{H}}$ & $\log \left(L_{\mathrm{X}_{\mathrm{INT}}}\right)$ & Rem. \\
\hline $137 \mathrm{~A}$ & 31612.9 & -550847 & 1.21 & 17.55 & 19.54 & $\bar{B}$ & 1.721 & 111 & 43.75 & -24.90 & 2.26 & $17.4_{-102}^{+16.7}$ & 44.42 & 1 \\
\hline $160 \mathrm{~A}$ & 31643.5 & -552240 & 6.41 & - & 22.34 & - & - & - & - & - & - & - & - & \\
\hline 160B & 31644.3 & -552243 & 1.43 & - & 21.96 & $\mathrm{~N}$ & 1.020 & 100 & 43.10 & - & - & - & - & \\
\hline 289A & 31647.7 & -551010 & 4.24 & - & 23.01 & - & - & - & - & - & - & - & - & \\
\hline $315 \mathrm{~A}$ & 31345.5 & -550448 & 6.50 & 15.92 & 20.09 & $\mathrm{~S}$ & 0.000 & 011 & - & - & - & - & - & $\mathrm{C}$ \\
\hline $315 B$ & 31346.0 & -550446 & 4.32 & - & 23.06 & $\mathrm{~N}$ & 0.836 & 110 & 42.93 & - & - & - & - & $\mathrm{C}$ \\
\hline $331 \mathrm{~A}$ & 31636.2 & -550932 & 2.72 & 19.73 & 22.13 & B & 1.876 & 111 & 43.89 & -22.53 & 1.80 & $0.52_{-0.52}^{+0.69}$ & 44.79 & 2,5 \\
\hline $341 \mathrm{~A}$ & 31338.2 & -552138 & 8.00 & 19.48 & 20.64 & B & 2.784 & 1 - - & 44.69 & -24.81 & 1.90 & - & - & 2 \\
\hline $366 \mathrm{~A}$ & 31538.8 & -552003 & 2.75 & 15.50 & 18.87 & $\mathrm{~S}$ & 0.00 & 011 & - & - & - & - & - & \\
\hline $386 \mathrm{~A}$ & 31339.9 & -550721 & 5.11 & 17.70 & 20.22 & G & 0.189 & 1 - - & 41.59 & - & - & $0.00_{-0.00}^{+0.17}$ & 41.59 & 3 \\
\hline $405 \mathrm{~A}$ & 31523.8 & -551759 & 5.65 & 10.96 & 13.14 & $\mathrm{~S}$ & 0.000 & 1 - - & - & - & - & - & - & \\
\hline $414 \mathrm{~A}$ & 31506.4 & -553312 & 2.36 & - & - & B & 2.058 & 1 - - & 44.49 & - & - & - & - & 2 \\
\hline $430 \mathrm{~A}$ & 31338.1 & -550706 & 6.86 & 17.03 & 20.04 & $\mathrm{~N}$ & 0.390 & 1 - - & 42.21 & - & - & $0.14_{-014}^{+0.27}$ & 42.34 & 3 \\
\hline $430 \mathrm{~B}$ & 31339.0 & -550714 & 7.64 & 18.52 & 20.66 & $\mathrm{~N}$ & 0.391 & 0 - - & - & - & - & - & - & 3 \\
\hline $432 \mathrm{~A}$ & 31554.9 & -550805 & 4.20 & 19.19 & - & - & - & - & - & - & - & - & - & \\
\hline $442 \mathrm{~A}$ & 31341.4 & -550033 & 5.91 & 19.23 & 22.16 & $\mathrm{~N}$ & 0.460 & 010 & - & - & - & - & - & \\
\hline $442 B$ & 31341.5 & -550037 & 4.60 & 18.31 & 22.01 & - & - & - & - & - & - & - & & \\
\hline $454 \mathrm{~A}$ & 31623.8 & -551031 & 1.82 & - & - & - & - & - & - & - & - & - & - & \\
\hline $522 \mathrm{~A}$ & 31613.1 & -550431 & 4.89 & 18.92 & 20.77 & B & 3.747 & 111 & 44.47 & -25.83 & 1.94 & - & - & \\
\hline 529A & 31622.4 & -551218 & 1.83 & 19.03 & - & - & - & - & - & - & - & - & - & \\
\hline $553 \mathrm{~A}$ & 31518.8 & -551723 & 7.40 & 18.78 & 19.90 & $\mathrm{~N}$ & 0.231 & 010 & - & - & - & - & - & \\
\hline $564 \mathrm{~A}$ & 31555.4 & -551504 & 7.40 & - & 23.37 & $\mathrm{~N}$ & 0.633 & 011 & - & - & - & - & - & \\
\hline $600 \mathrm{~A}$ & 31503.9 & -551654 & 5.68 & 18.38 & 21.15 & $\mathrm{~N}$ & 0.452 & 110 & 42.16 & - & - & - & - & \\
\hline $612 \mathrm{~A}$ & 31606.9 & -550426 & 3.45 & - & 23.74 & $\mathrm{~N}$ & 0.409 & 110 & 42.32 & - & - & - & - & \\
\hline $623 \mathrm{~A}$ & 31642.5 & -551707 & 2.56 & - & 20.18 & G & 0.073 & 110 & 40.76 & - & - & $0.01_{-001}^{+0.11}$ & 40.79 & \\
\hline 677A & 31640.4 & -551929 & 7.85 & - & 21.93 & $\mathrm{~N}$ & 0.131 & 010 & - & - & - & - & - & \\
\hline 677B & 31641.5 & -551937 & 4.76 & - & - & $\mathrm{N}$ & 1.354 & 101 & 43.41 & - & - & - & - & \\
\hline
\end{tabular}

137A: Broad absorption line quasar.

315A and 315B: As counterpart candidates for X-ray source 315, an M star (315A) and a NELG (315B) are found. The hardness ratios of 315 indicated an obscured X-ray object. Therefore, we regarded 315B as the identification. 
M. Krumpe et al.: The XMM-Newton survey in the Marano field. I., Online Material p 10

Table B.2. X-ray source list for marginal X-ray sources. The explanation of the individual columns is given in Appendix A.

\begin{tabular}{ccccccccc}
\hline \hline$(1)$ & $\begin{array}{c}(2) \\
\text { ID }\end{array}$ & $\begin{array}{c}(3) \\
\text { CR } \\
\text { XMMU J... }\end{array}$ & $\begin{array}{c}(4) \\
\text { ML }\end{array}$ & $\begin{array}{c}(5) \\
\left.\mathrm{ks}^{-1}\right] \\
{[\mathrm{arcsec}]}\end{array}$ & $\begin{array}{c}(6) \\
H R 1\end{array}$ & $\begin{array}{c}(7) \\
H R 2\end{array}$ & $\begin{array}{c}(8) \\
H R 3\end{array}$ & $\begin{array}{c}(9) \\
\text { Flux } \\
{\left[10^{-14} \mathrm{erg} \mathrm{s}^{-1} \mathrm{~cm}^{-2}\right]}\end{array}$ \\
\hline 137 & $031612.8-550846$ & $1.8 \pm 0.6$ & 2.7 & 2.09 & $-0.003 \pm 0.890$ & $0.289 \pm 0.683$ & $-0.022 \pm 0.636$ & 0.285 \\
160 & $031644.2-552242$ & $1.4 \pm 0.8$ & 3.1 & 2.29 & - & - & - & 0.228 \\
289 & $031648.0-551013$ & $2.4 \pm 0.8$ & 4.1 & 2.10 & $1.000 \pm 0.490$ & $-1.000 \pm 0.187$ & - & 0.390 \\
315 & $031346.2-550450$ & $1.6 \pm 0.6$ & 2.6 & 2.31 & - & $0.589 \pm 0.565$ & $-1.000 \pm 0.929$ & 0.252 \\
331 & $031636.5-550929$ & $1.9 \pm 0.7$ & 3.6 & 2.08 & $1.000 \pm 0.823$ & $0.153 \pm 0.745$ & $-0.278 \pm 0.757$ & 0.313 \\
341 & $031337.8-552144$ & $4.8 \pm 1.9$ & 3.2 & 15.08 & $-1.000 \pm 0.365$ & - & - & 0.767 \\
366 & $031538.8-552001$ & $1.5 \pm 0.5$ & 2.6 & 2.26 & $0.263 \pm 0.894$ & $0.146 \pm 0.695$ & $-0.198 \pm 0.734$ & 0.248 \\
386 & $031339.5-550724$ & $2.4 \pm 0.7$ & 2.5 & 2.51 & - & - & $-1.000 \pm 0.373$ & 0.382 \\
405 & $031523.3-551756$ & $0.9 \pm 0.3$ & 1.1 & 2.59 & $1.000 \pm 0.774$ & $0.271 \pm 0.649$ & $-0.887 \pm 0.667$ & 0.139 \\
414 & $031506.5-553314$ & $6.1 \pm 2.3$ & 2.6 & 2.49 & - & $-1.000 \pm 0.934$ & - & 0.991 \\
430 & $031339.0-550706$ & $1.9 \pm 0.6$ & 4.8 & 1.97 & $0.738 \pm 0.432$ & $-0.946 \pm 0.348$ & - & 0.305 \\
432 & $031555.1-550801$ & $1.7 \pm 0.5$ & 3.2 & 2.08 & $1.000 \pm 0.305$ & $-0.043 \pm 0.374$ & $-1.000 \pm 0.650$ & 0.272 \\
442 & $031341.0-550038$ & $3.2 \pm 1.0$ & 2.4 & 2.51 & $0.557 \pm 0.472$ & $-0.278 \pm 0.435$ & $-1.000 \pm 0.482$ & 0.518 \\
454 & $031623.7-551031$ & $2.7 \pm 0.8$ & 3.3 & 2.72 & $0.364 \pm 0.338$ & $-0.689 \pm 0.373$ & $0.229 \pm 0.859$ & 0.429 \\
522 & $031613.7-550430$ & $1.4 \pm 0.7$ & 1.3 & 2.84 & $-0.176 \pm 0.775$ & - & - & 0.223 \\
529 & $031622.3-551217$ & $2.1 \pm 0.6$ & 2.4 & 2.58 & $-0.479 \pm 0.401$ & $0.141 \pm 0.586$ & $-0.340 \pm 0.703$ & 0.334 \\
553 & $031518.0-551722$ & $1.4 \pm 0.4$ & 1.4 & 2.44 & $0.953 \pm 0.276$ & $-0.020 \pm 0.336$ & $-0.319 \pm 0.411$ & 0.224 \\
564 & $031554.9-551459$ & $1.4 \pm 0.4$ & 2.3 & 2.37 & $1.000 \pm 0.391$ & $-0.193 \pm 0.363$ & $-1.000 \pm 0.580$ & 0.230 \\
581 & $031638.3-551901$ & $1.7 \pm 0.7$ & 4.2 & 2.44 & - & - & $1.000 \pm 0.534$ & 0.266 \\
600 & $031503.3-551656$ & $1.2 \pm 0.4$ & 3.1 & 2.49 & $-0.347 \pm 0.390$ & $-1.000 \pm 0.511$ & - & 0.191 \\
612 & $031606.8-550423$ & $2.2 \pm 0.6$ & 4.2 & 2.33 & $-0.340 \pm 0.777$ & $0.352 \pm 0.781$ & $-0.676 \pm 0.809$ & 0.347 \\
623 & $031642.3-551707$ & $2.7 \pm 0.8$ & 4.1 & 2.56 & $1.000 \pm 0.277$ & $-0.407 \pm 0.505$ & - & 0.433 \\
677 & $031641.2-551934$ & $1.5 \pm 0.7$ & 3.6 & 3.35 & - & $1.000 \pm 0.710$ & - & 0.234 \\
\hline
\end{tabular}




\section{Appendix C: Optical and X-ray images and spectra of X-ray classifications}

\section{C.1. Optical images}

The optical images show a $30^{\prime \prime} \times 30^{\prime \prime}$ section that is centred on the X-ray position (except for images next to the edge of the CCD). The cut levels are set differently for each image. The black circle shows an $1 \sigma$-X-ray position error including a 2 " systematic error. Black horizontal markers represent the position of the spectroscopically classified object. The nominal orientation of the images is North facing upwards and east to the left (exceptions are marked).

Images were generated using VLT FORS pre-images, WFI- $R$-band images, or DSSII-images, in this order of availability. The astrometric correction of VLT FORS pre-images was performed as a 2-stage process, since USNO-stars or stars from similarly well-calibrated cataloges were found to be too sparse or were saturated. We therefore first generated a stellar catalogue of WFI objects (calibrated with the USNO) using this as input catalogue for astrometric calibration of the VLT images. We typically used stars of the order of 10 that were equally distributed over the field of view and reached an rms accurracy of $\sim 0.2^{\prime \prime}(0.7$ pixels $)$.

\section{C.2. X-ray images}

The X-ray images show a $\sim 32^{\prime \prime} \times 32^{\prime \prime}$ section which is nearly centred on the X-ray position. The cut levels are set differently for every image. The black circle shows an $1 \sigma$-X-ray position error ( 2 " systematic error). Black horizontal markers represent the position of the spectroscopically classsified object. The nominal orientation of the X-ray images is North facing upwards and East to the left.

\section{C.3. Labelling of spectra}

All spectra are in flux units of $10^{-18} \mathrm{erg} \mathrm{cm}^{-2} \mathrm{~s}^{-1} \AA^{-1}$. The black solid line represents the spectrum, the green solid line the error spectrum. Red markers indicate emission lines, blue markers absorption lines. There are exeptions in a few spectra. If no optical spectrum is shown the redshift and classification is taken from the literature.

\section{C.4. Comment}

Below every row of opticaland X-ray images and optical spectrum a comment for the shown objects is given. This comment states the classification number of the possible counterpart object and the corresponding redshift. If the redshift is only determined by one identification feature in the optical spectra, an additional comment ": @<identification feature>" is shown. The colon marks the uncertainty of the redshift (these objects have a redshift flag of " 0 " in Table 8 (full paper) and Table B.1).
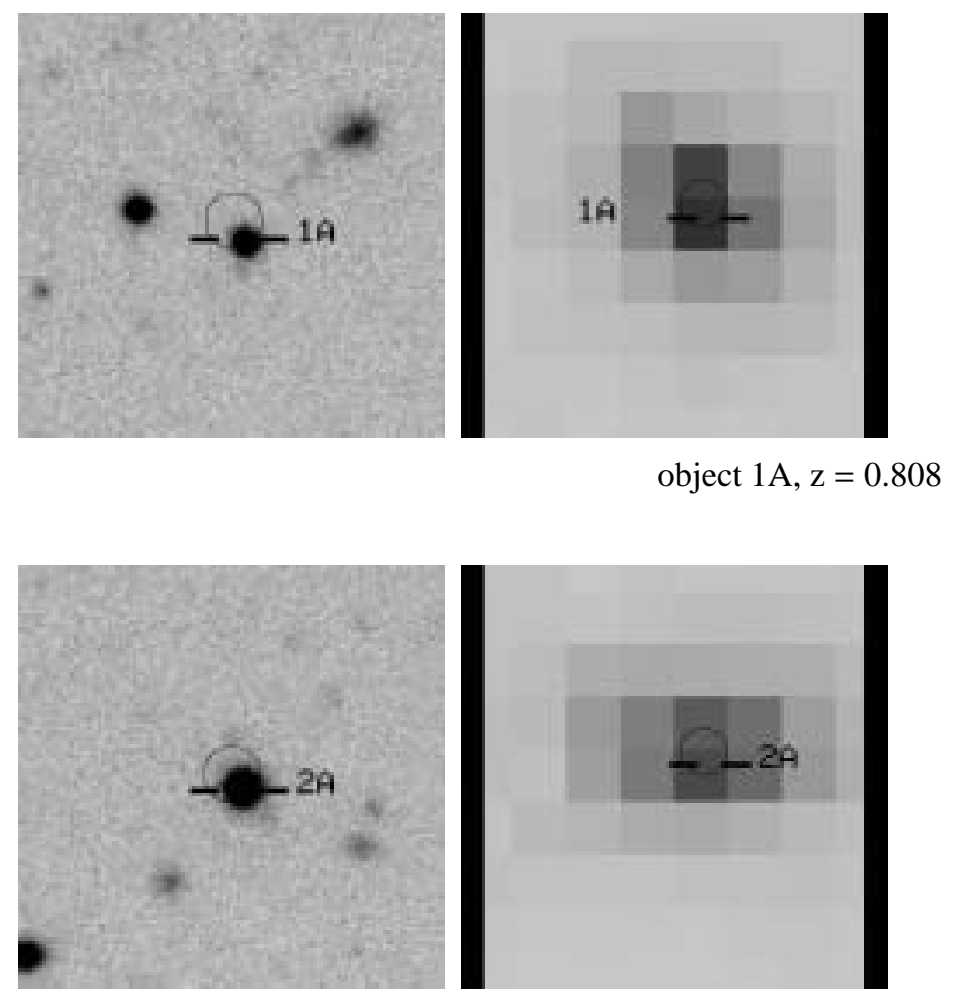

object $2 \mathrm{~A}, \mathrm{z}=0.464$ 
M. Krumpe et al.: The XMM-Newton survey in the Marano field. I., Online Material p 12
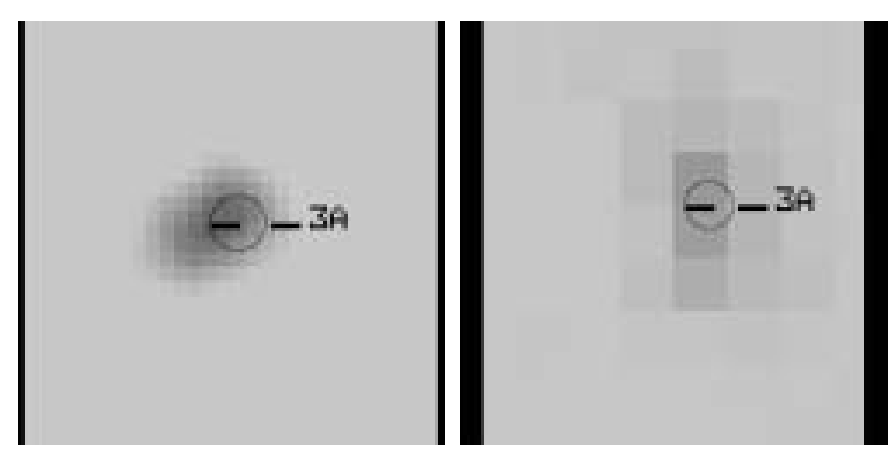

object $3 \mathrm{~A}, \mathrm{z}=0.406$
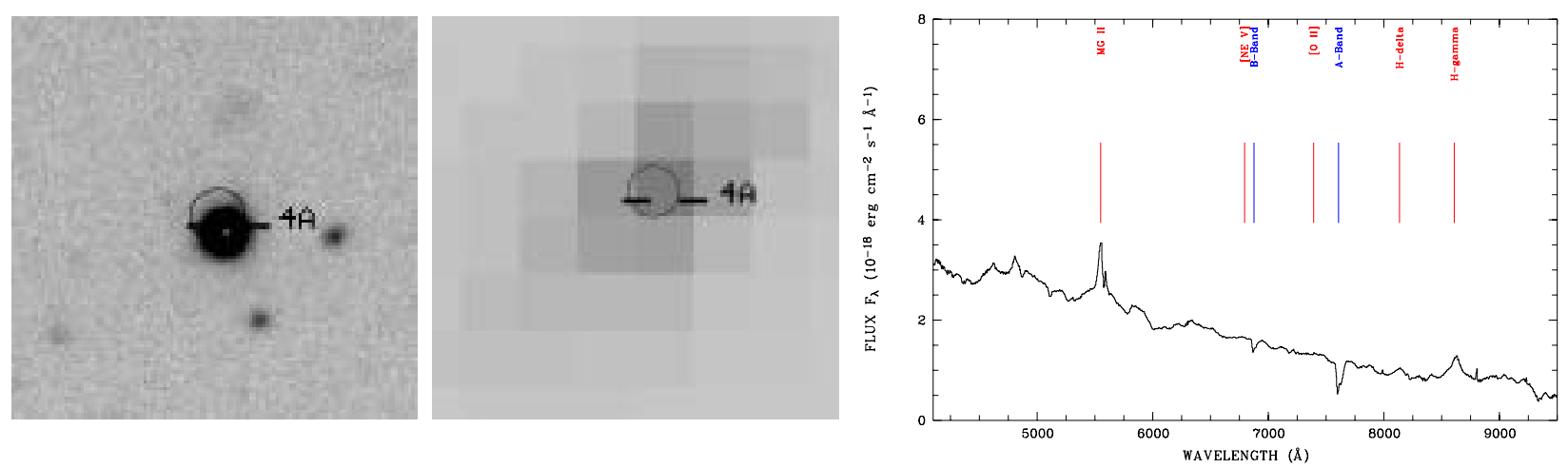

object $4 \mathrm{~A}, \mathrm{z}=0.987$
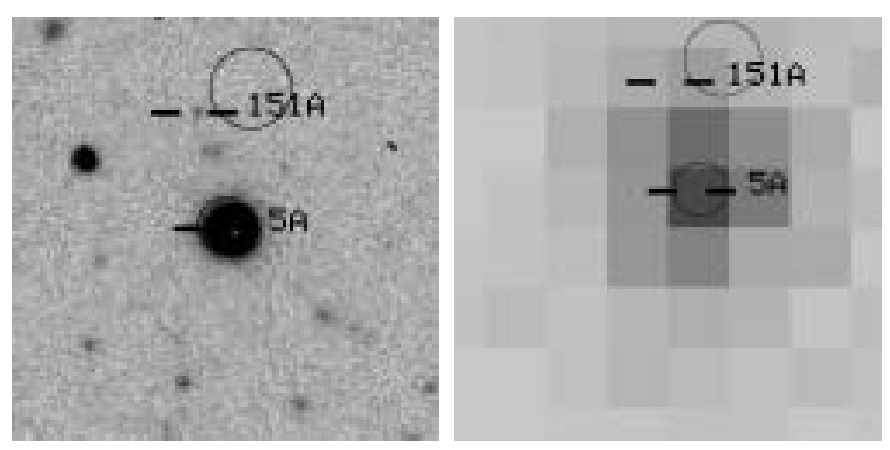

object $5 \mathrm{~A}, \mathrm{z}=2.531$
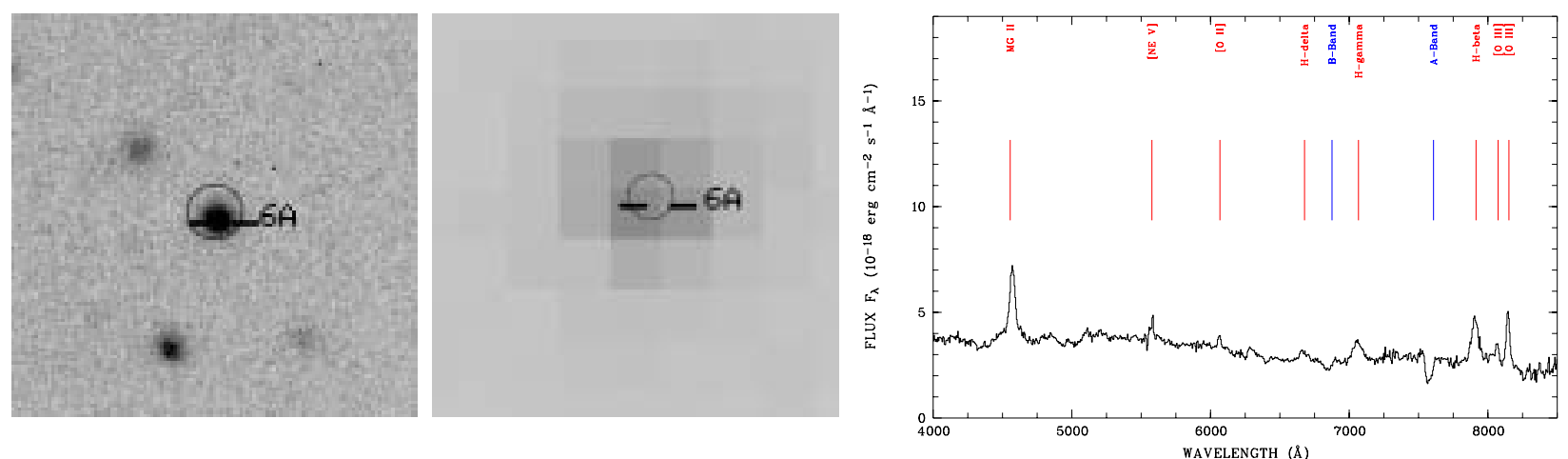

object $6 \mathrm{~A}, \mathrm{z}=0.636$ 
M. Krumpe et al.: The XMM-Newton survey in the Marano field. I., Online Material p 13
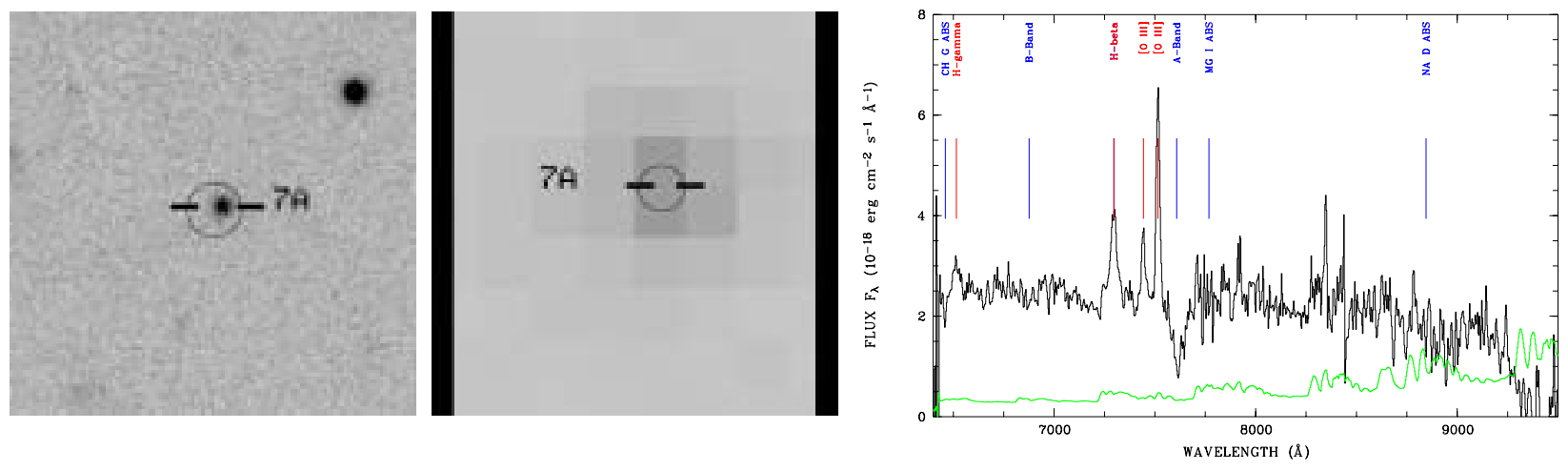

object $7 \mathrm{~A}, \mathrm{z}=0.501$
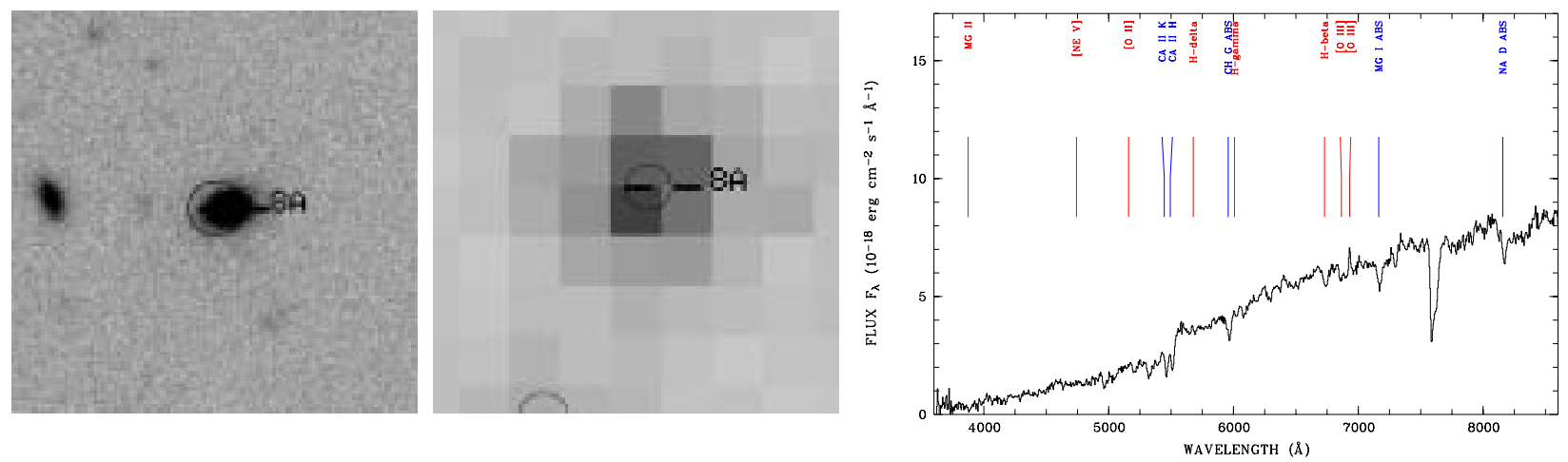

object $8 \mathrm{~A}, \mathrm{z}=0.387$
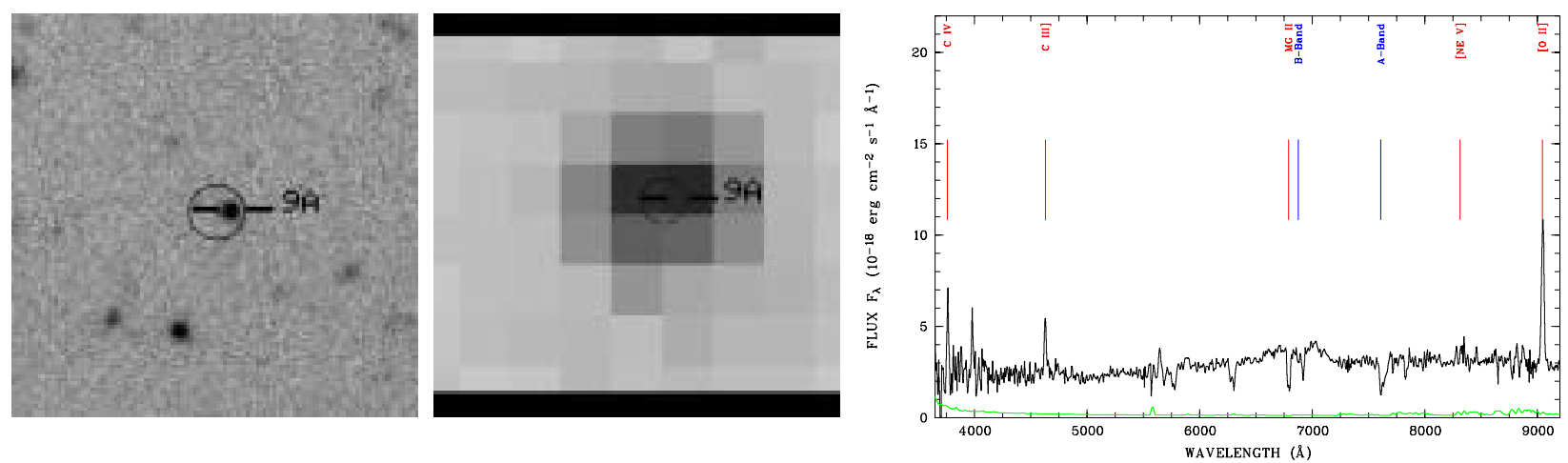

object $9 \mathrm{~A}, \mathrm{z}=1.427$
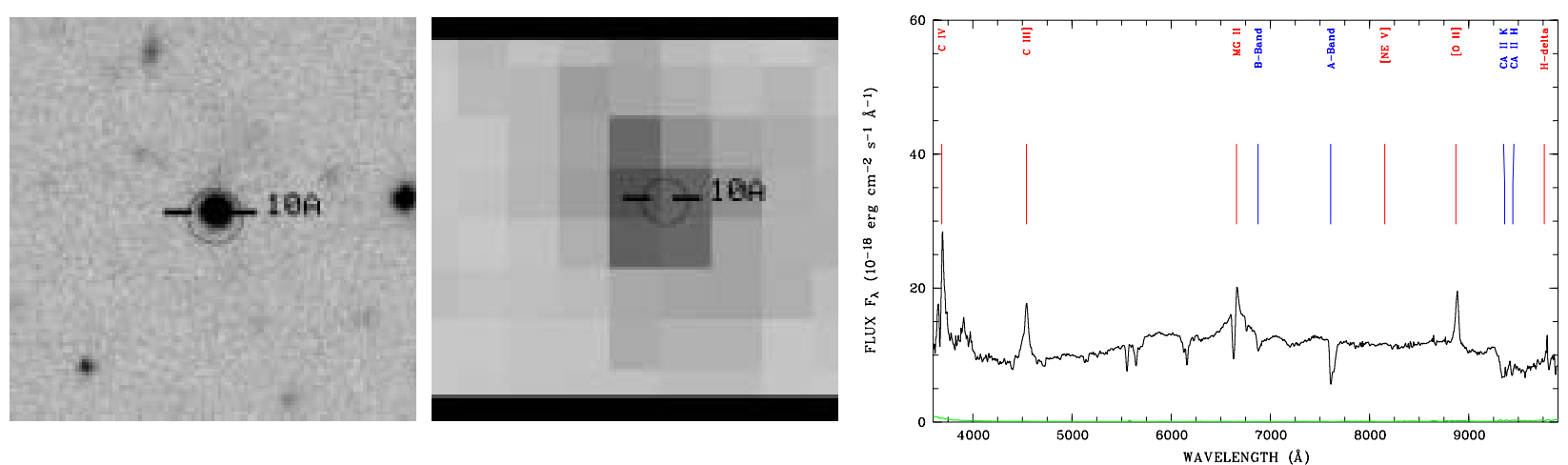

object 10A, $\mathrm{z}=1.378$ 
M. Krumpe et al.: The XMM-Newton survey in the Marano field. I., Online Material p 14
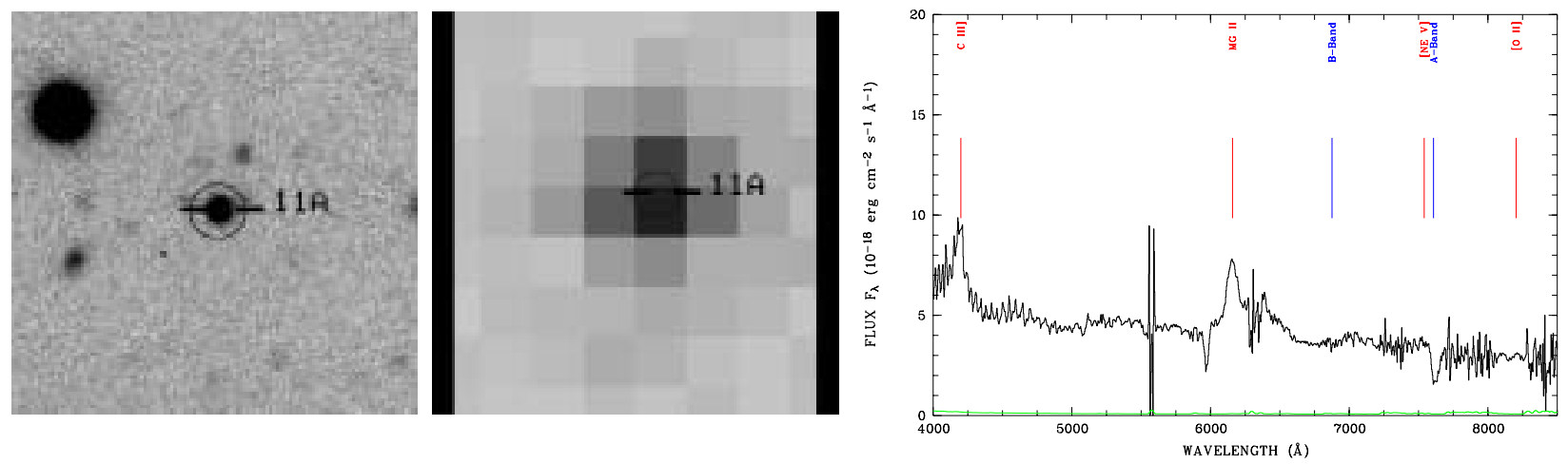

object $11 \mathrm{~A}, \mathrm{z}=1.192$
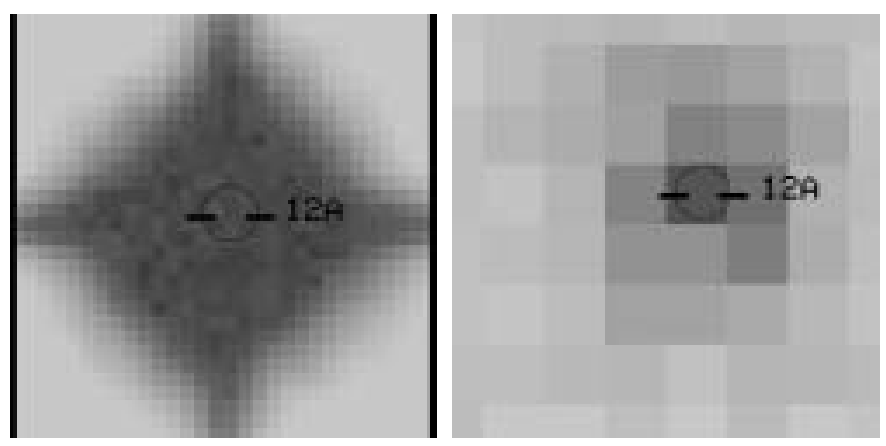

object $12 \mathrm{~A}, \mathrm{z}=0.000$
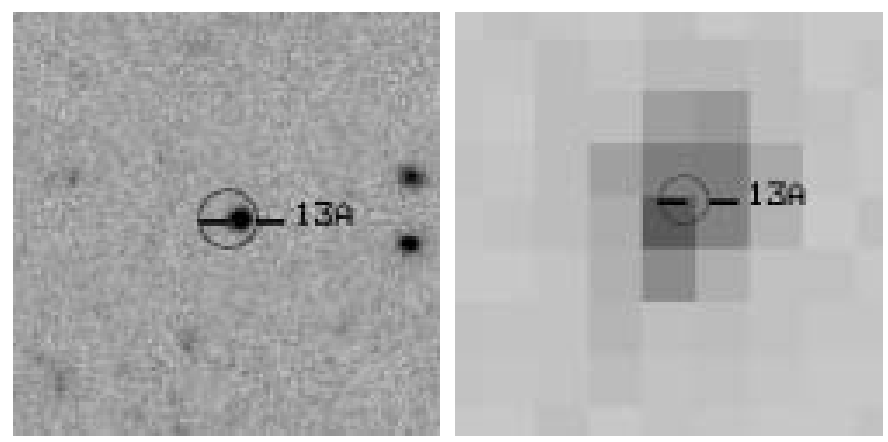

object $13 \mathrm{~A}, \mathrm{z}=0.500$
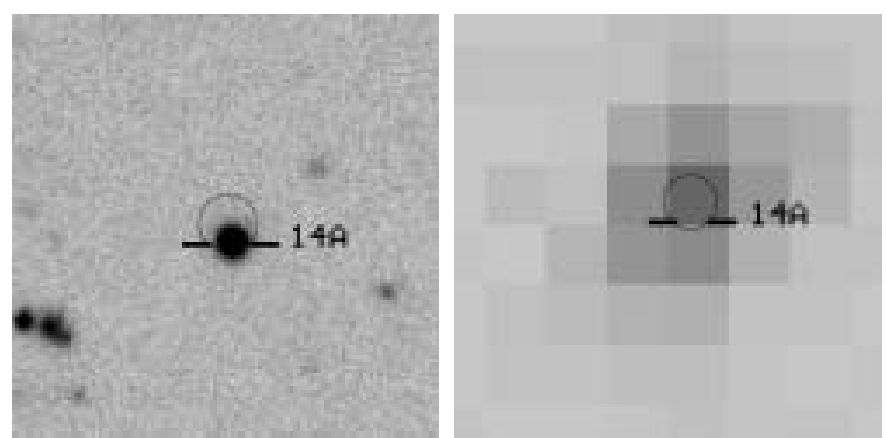

object $14 \mathrm{~A}, \mathrm{z}=0.854$ 
M. Krumpe et al.: The XMM-Newton survey in the Marano field. I., Online Material p 15
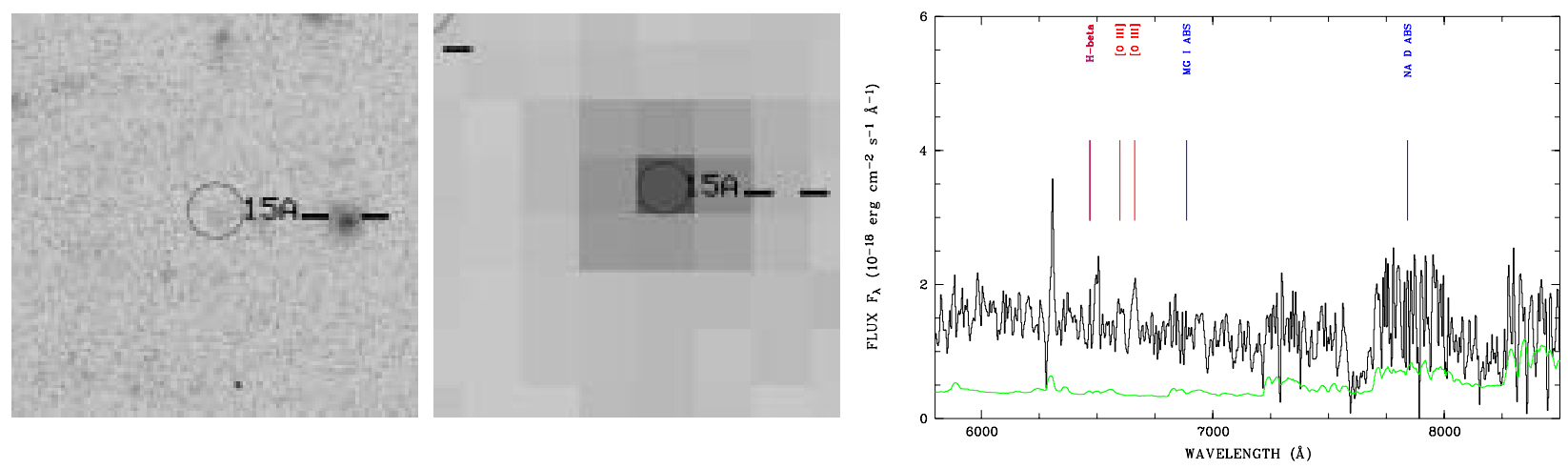

object 15A, z=0.331:@OIII
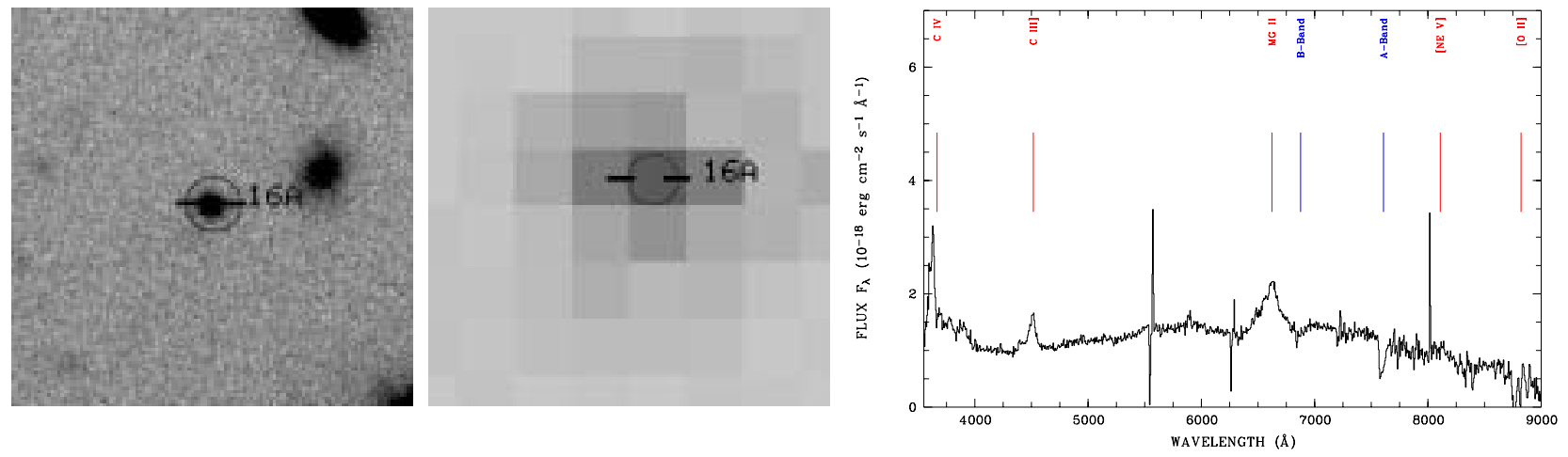

object $16 \mathrm{~A}, \mathrm{z}=1.374$
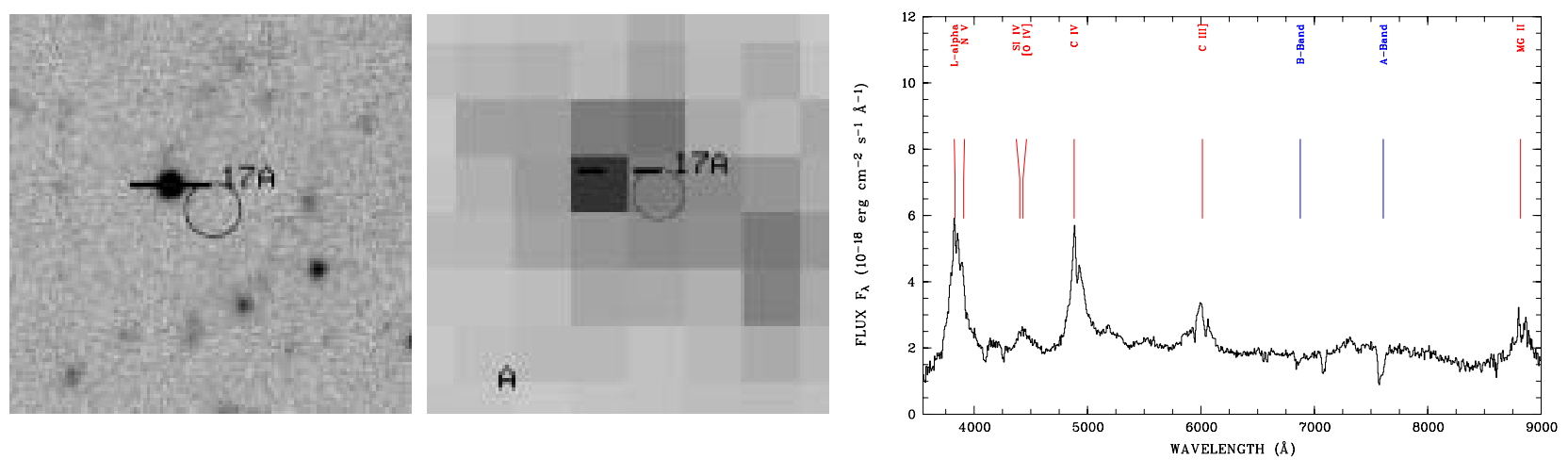

object $17 \mathrm{~A}, \mathrm{z}=2.161$
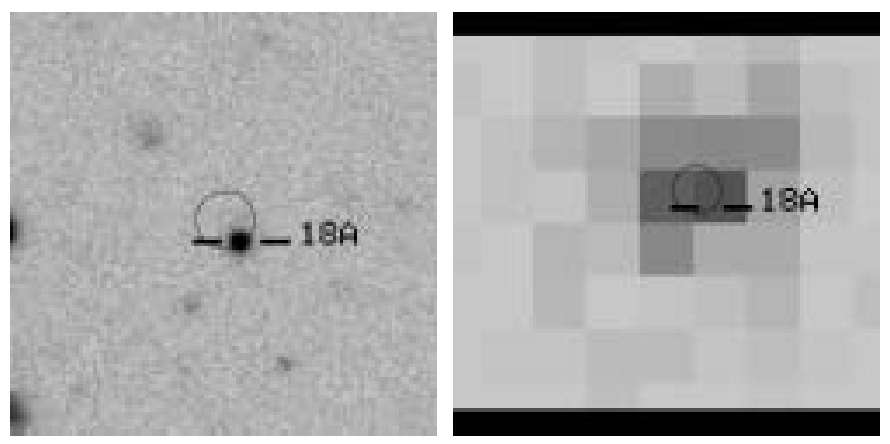

object $18 \mathrm{~A}, \mathrm{z}=0.614$ 
M. Krumpe et al.: The XMM-Newton survey in the Marano field. I., Online Material p 16
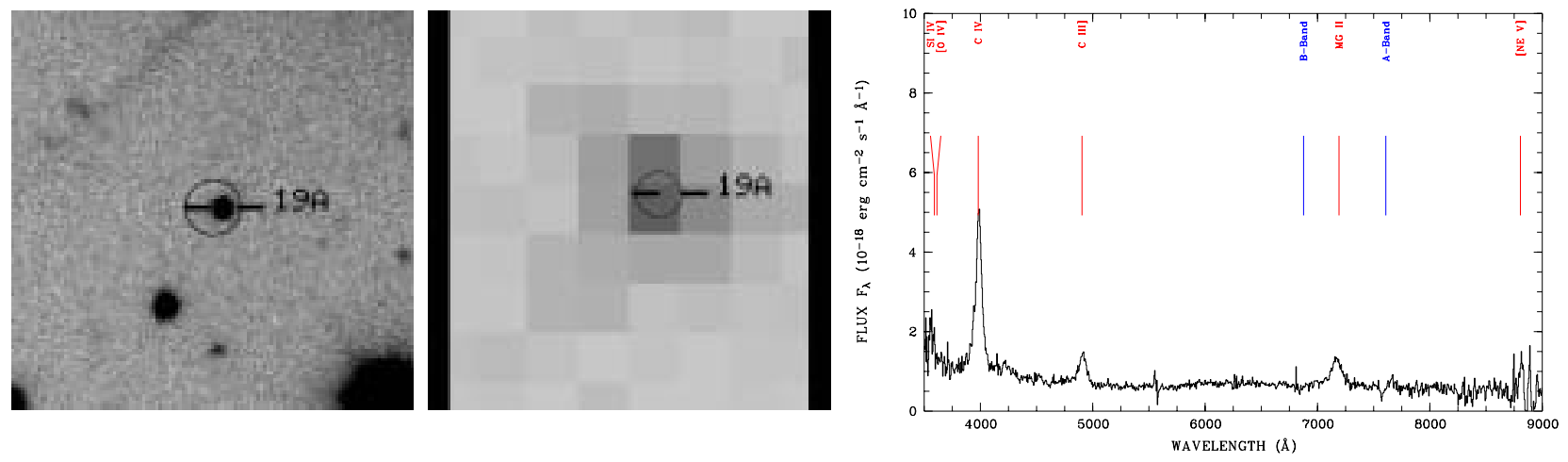

object 19A, $\mathrm{z}=1.573$
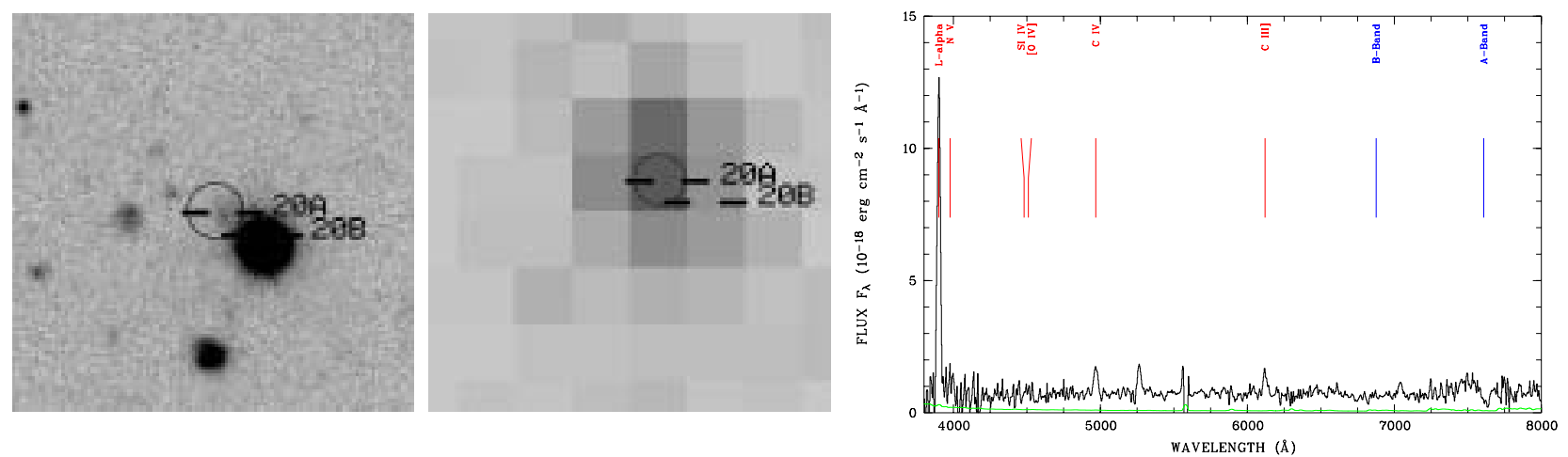

object $20 \mathrm{~A}, \mathrm{z}=2.207$
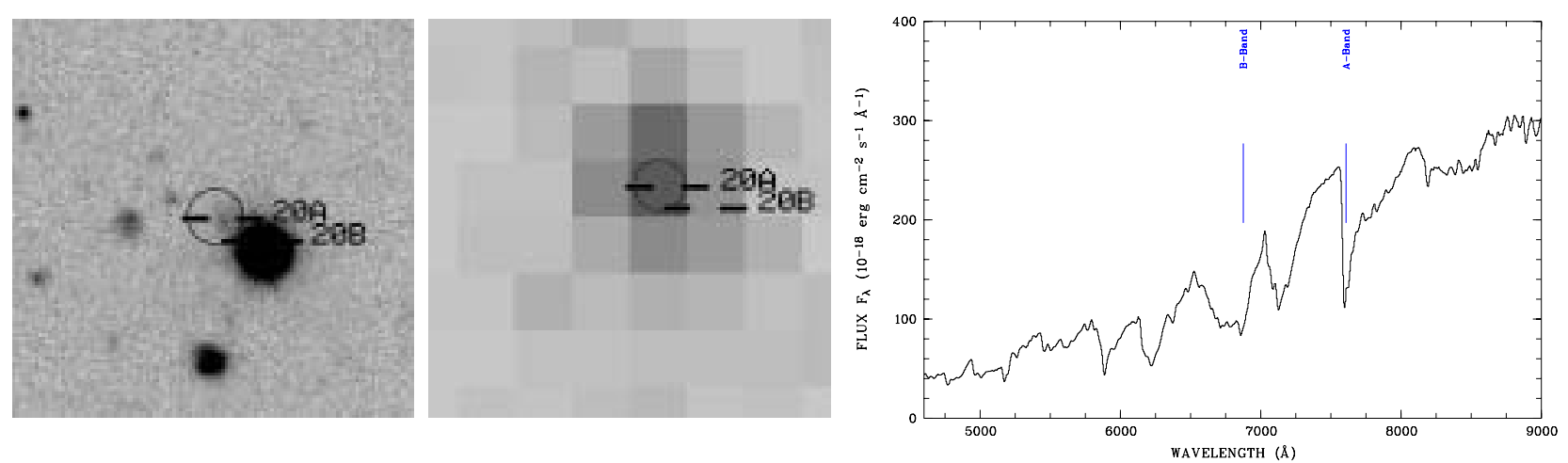

object $20 \mathrm{~B}, \mathrm{z}=0.000$
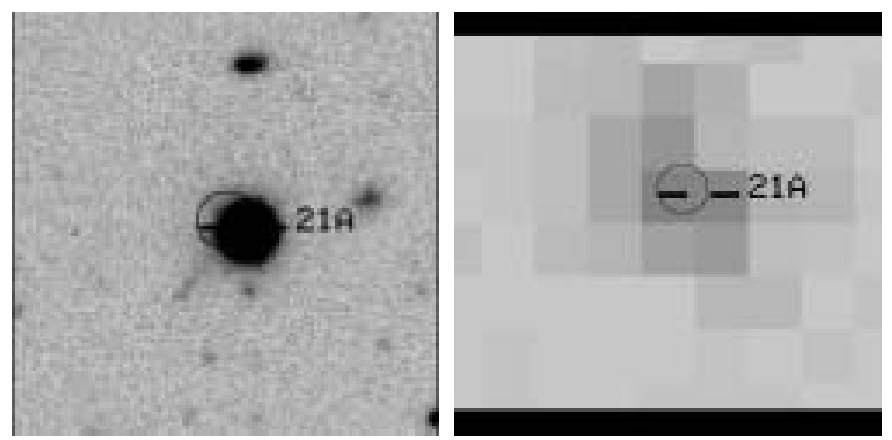

object $21 \mathrm{~A}, \mathrm{z}=0.000$ 
M. Krumpe et al.: The XMM-Newton survey in the Marano field. I., Online Material p 17
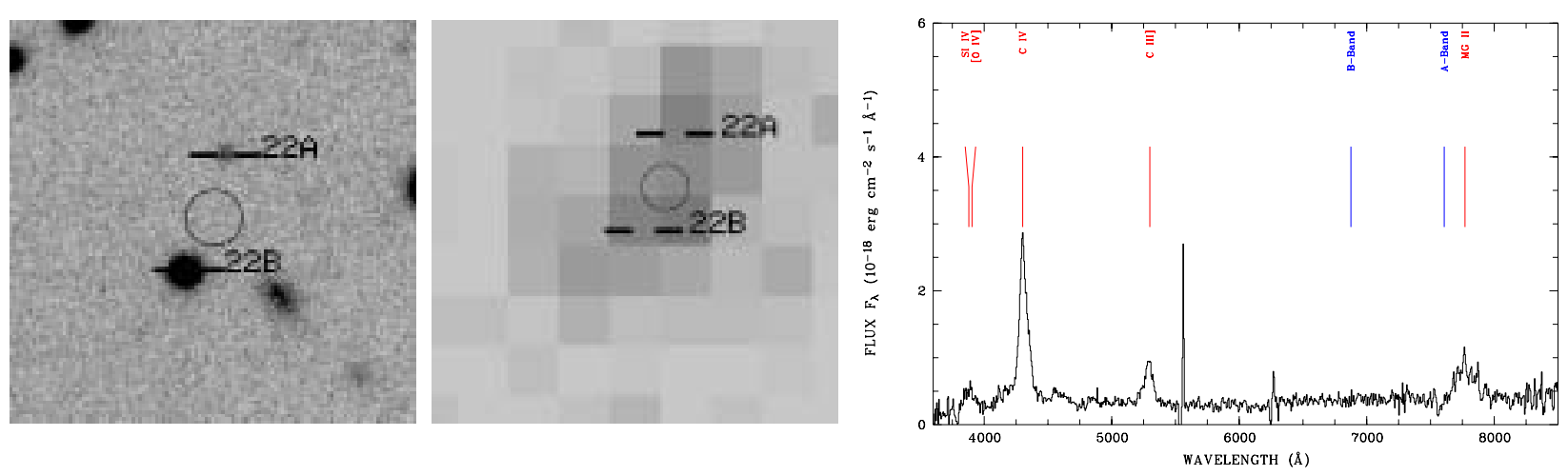

object $22 \mathrm{~A}, \mathrm{z}=1.78$
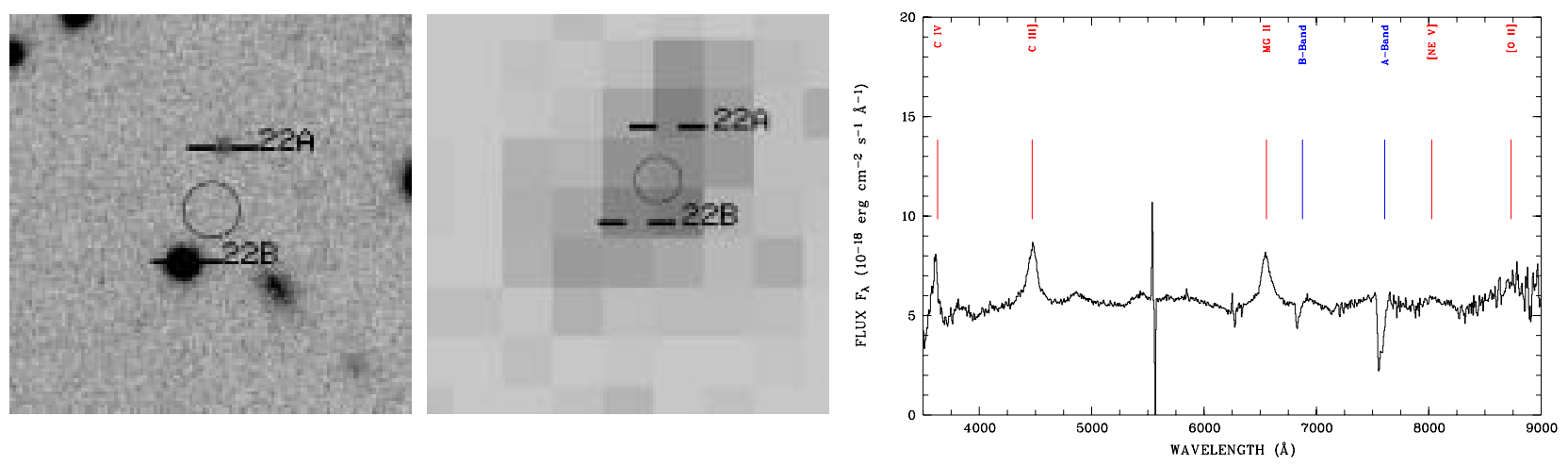

object $22 \mathrm{~B}, \mathrm{z}=1.353$
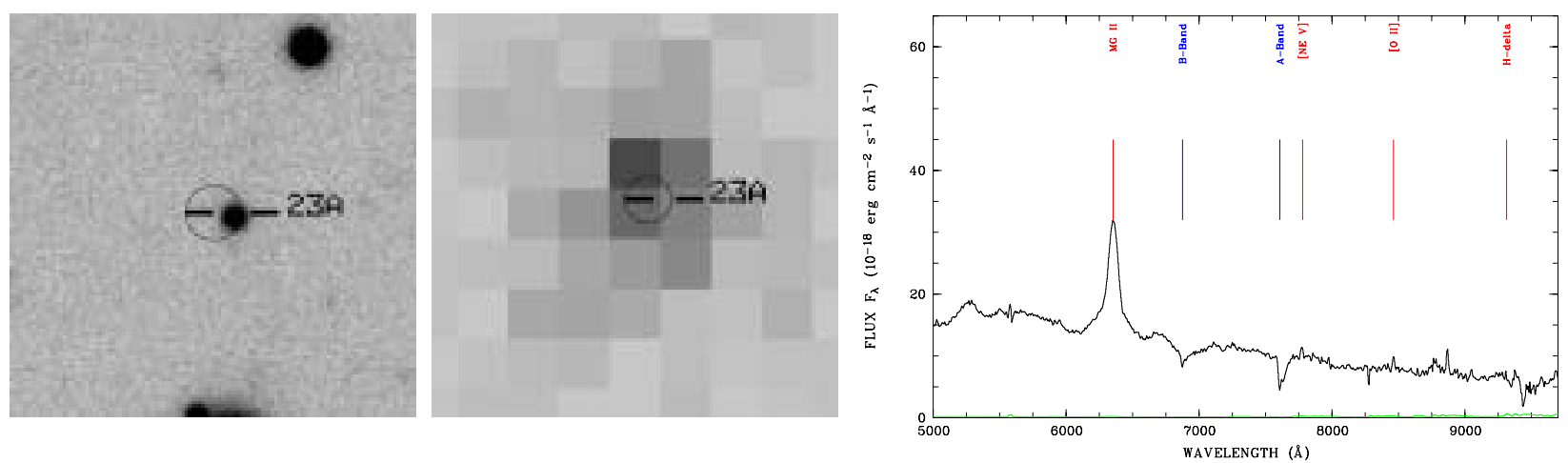

object $23 \mathrm{~A}, \mathrm{z}=1.271$
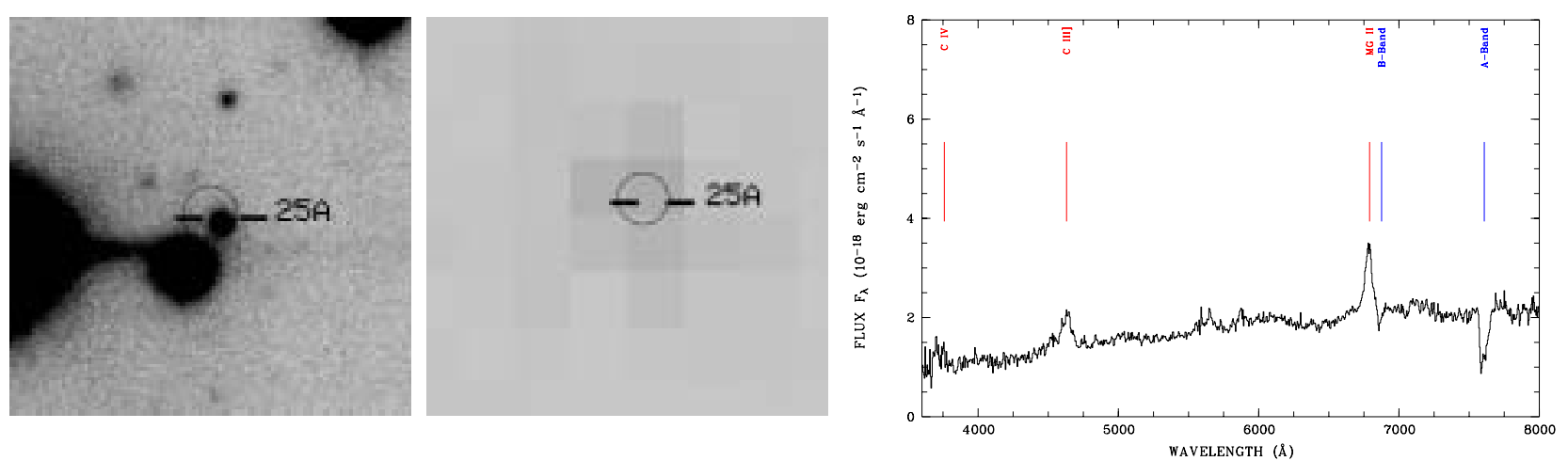

object $25 \mathrm{~A}, \mathrm{z}=1.430$ 
M. Krumpe et al.: The XMM-Newton survey in the Marano field. I., Online Material p 18
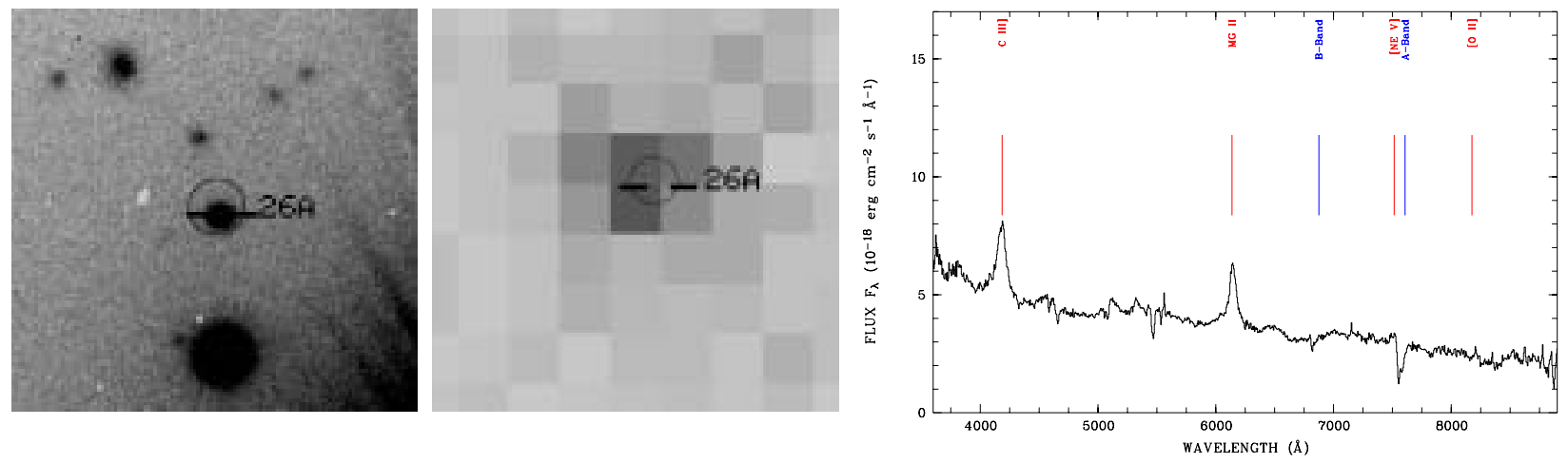

object $26 \mathrm{~A}, \mathrm{z}=1.19$
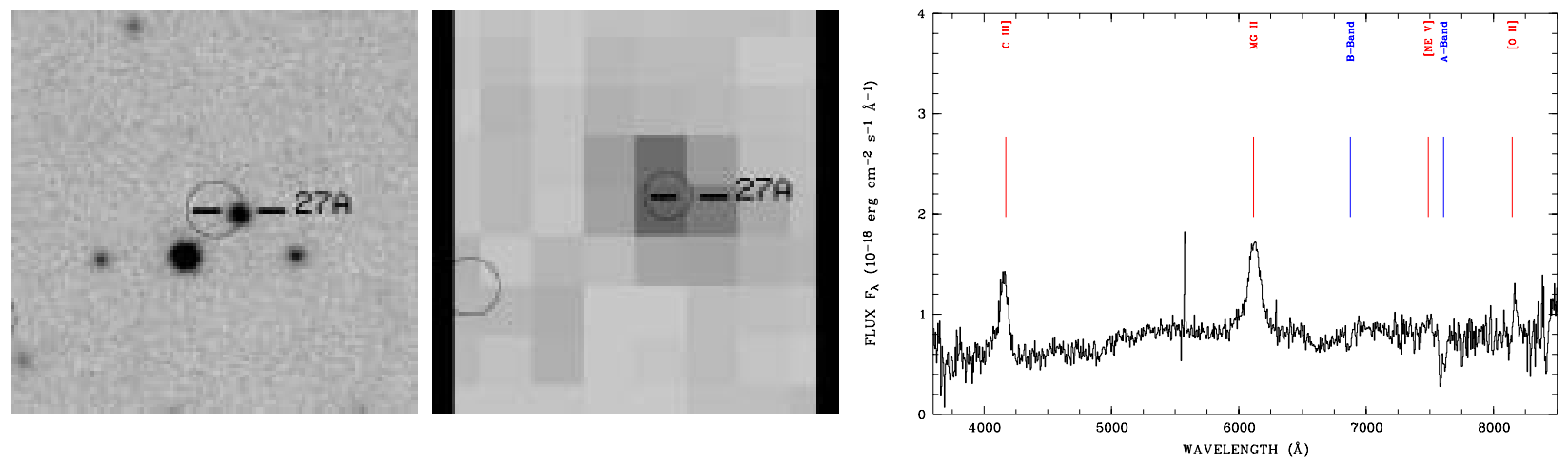

object $27 \mathrm{~A}, \mathrm{z}=1.190$
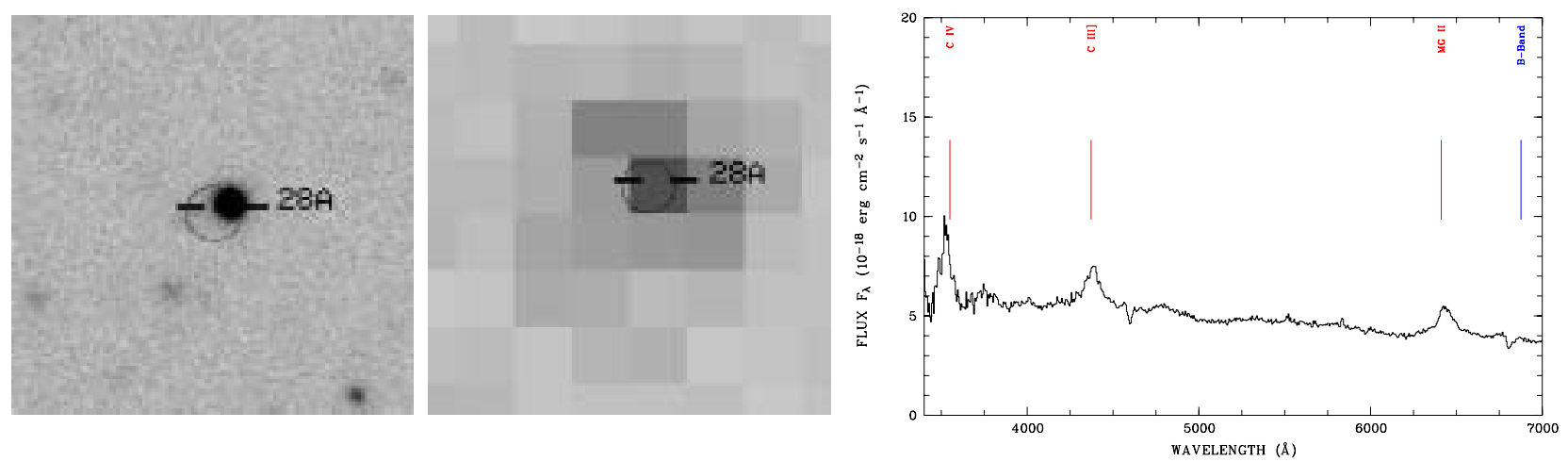

object $28 \mathrm{~A}, \mathrm{z}=1.315$
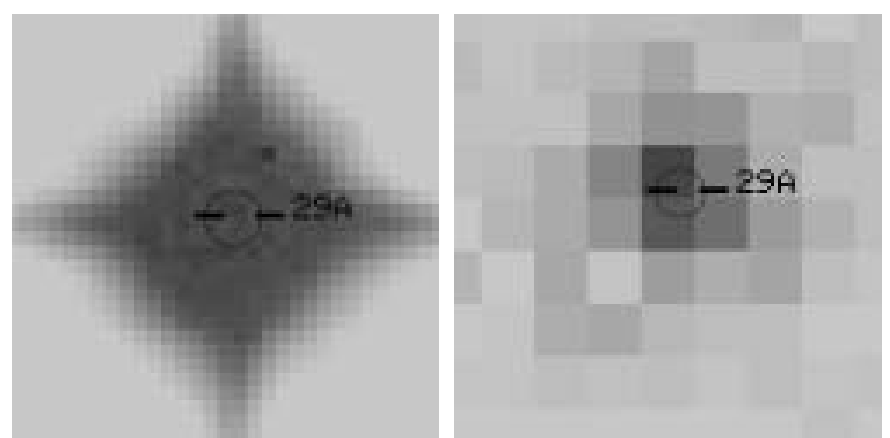

object $29 \mathrm{~A}, \mathrm{z}=0.000$ 
M. Krumpe et al.: The XMM-Newton survey in the Marano field. I., Online Material p 19
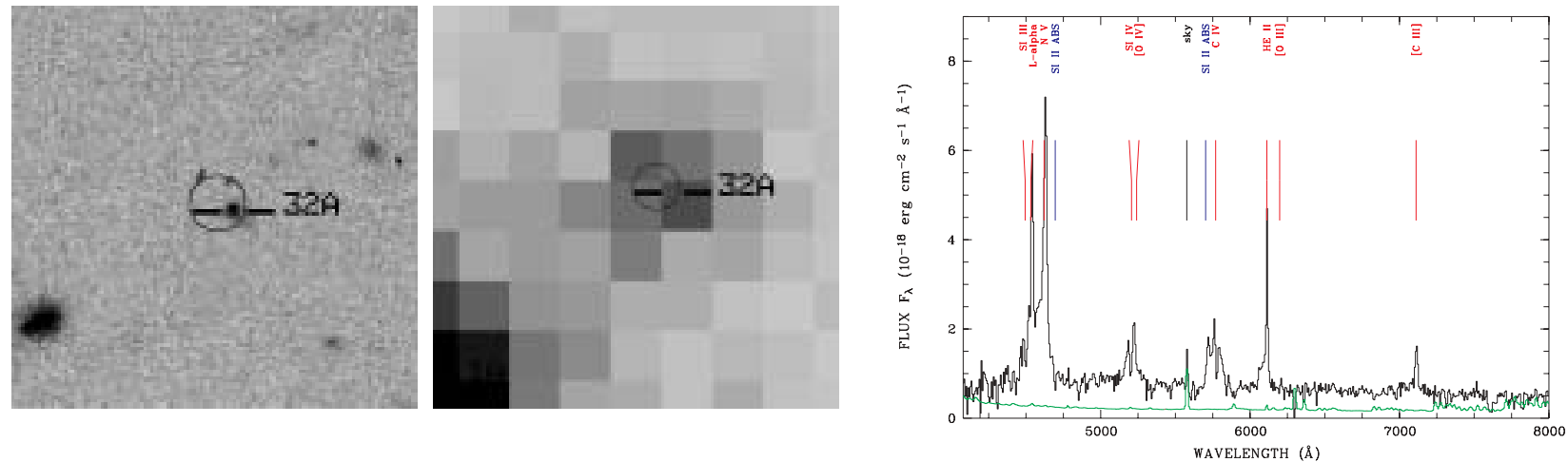

object $32 \mathrm{~A}, \mathrm{z}=2.727$
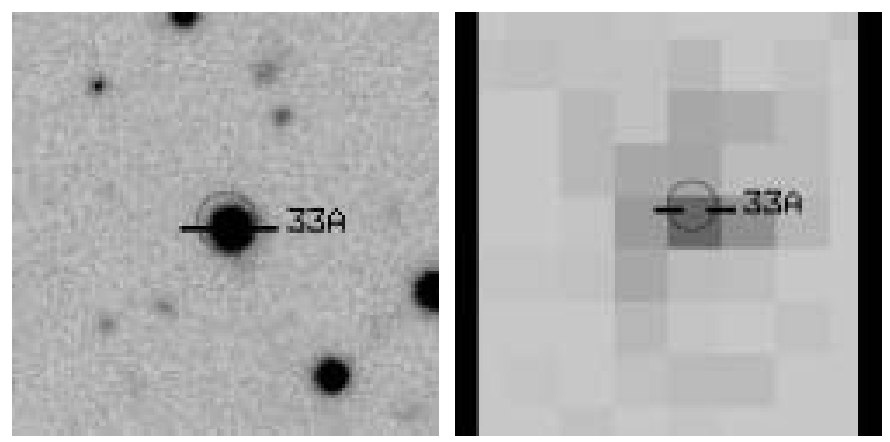

object $33 \mathrm{~A}, \mathrm{z}=2.166$
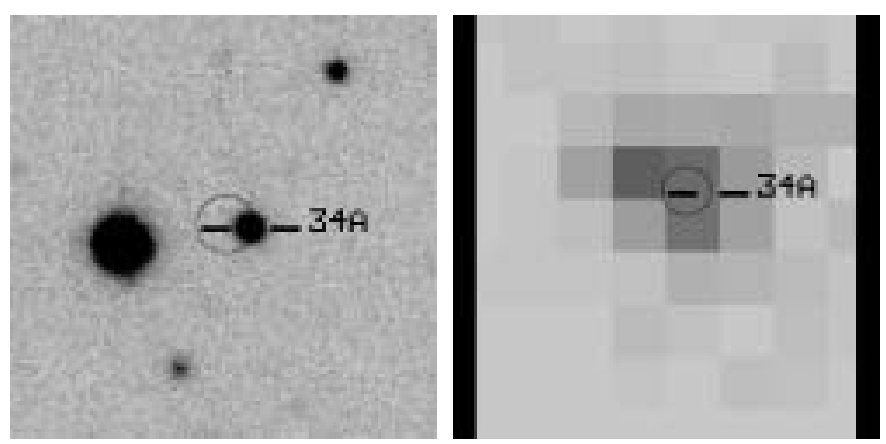

object $34 \mathrm{~A}, \mathrm{z}=0.983$
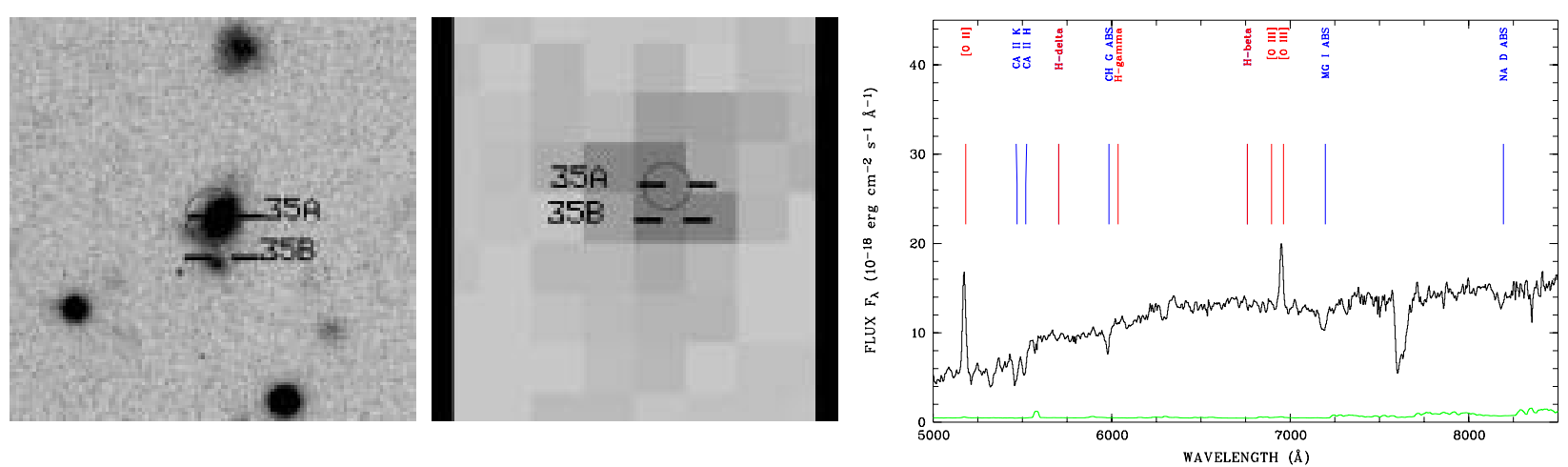

object $35 \mathrm{~A}, \mathrm{z}=0.391$ 
M. Krumpe et al.: The XMM-Newton survey in the Marano field. I., Online Material p 20
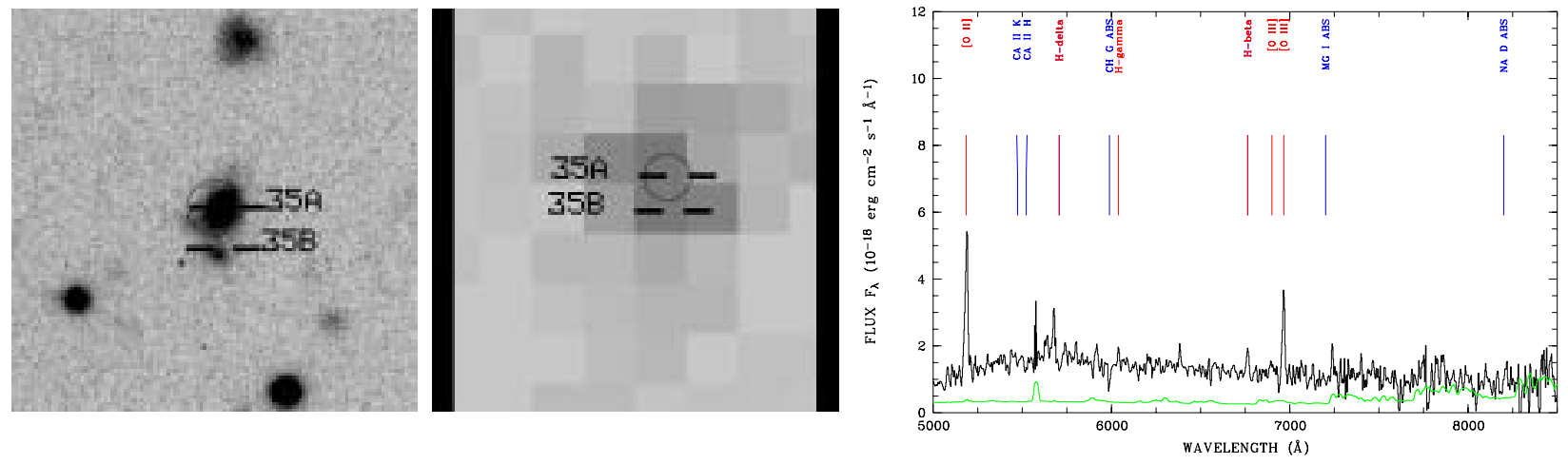

object $35 \mathrm{~B}, \mathrm{z}=0.391$
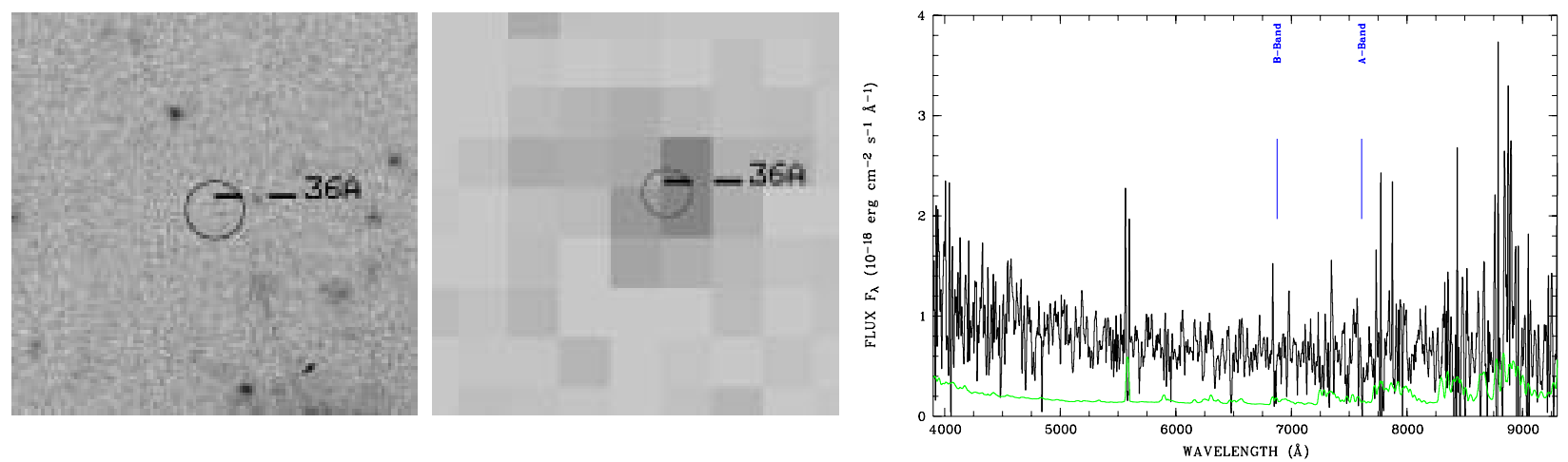

object 36A, no redshift, spectrum too noisy
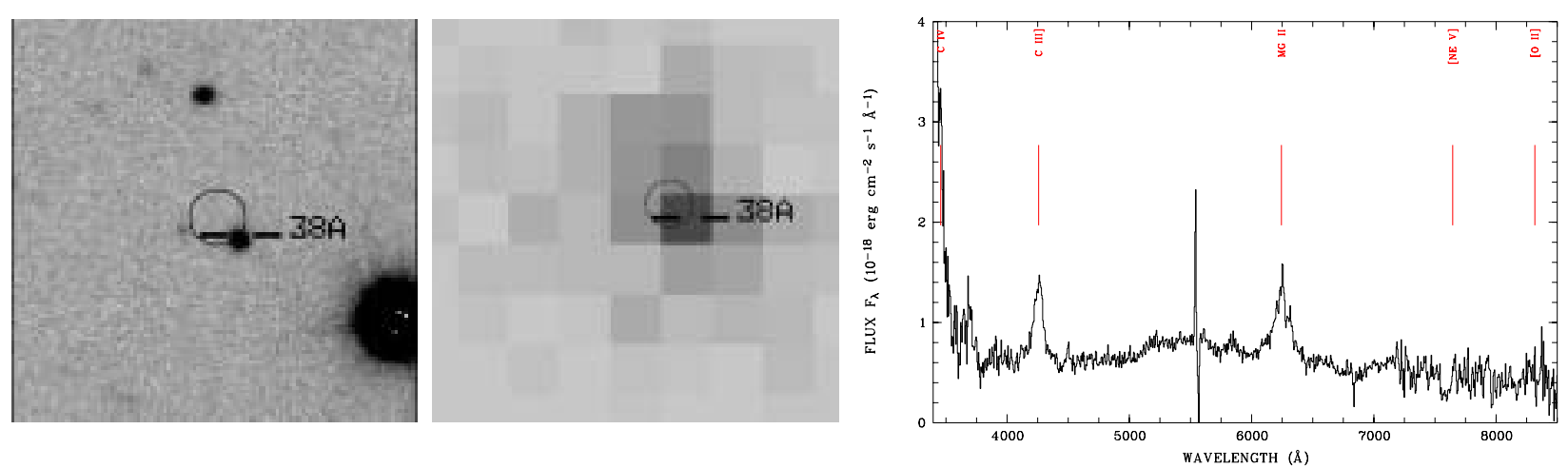

object $38 \mathrm{~A}, \mathrm{z}=1.254$
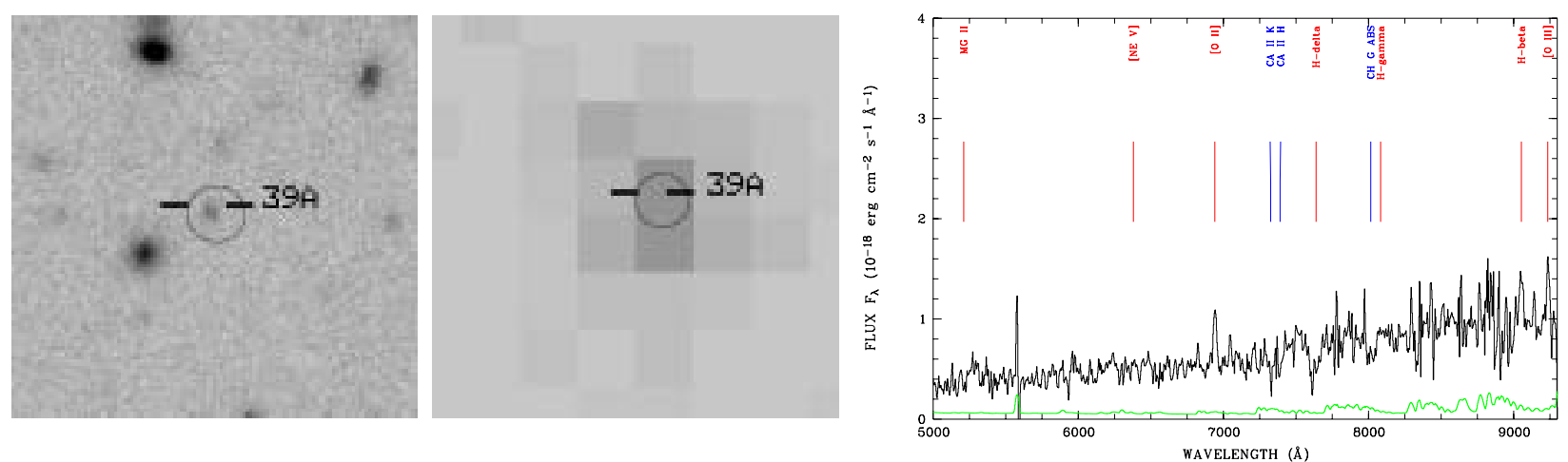

object $39 \mathrm{~A}, \mathrm{z}=0.862$ 
M. Krumpe et al.: The XMM-Newton survey in the Marano field. I., Online Material p 21
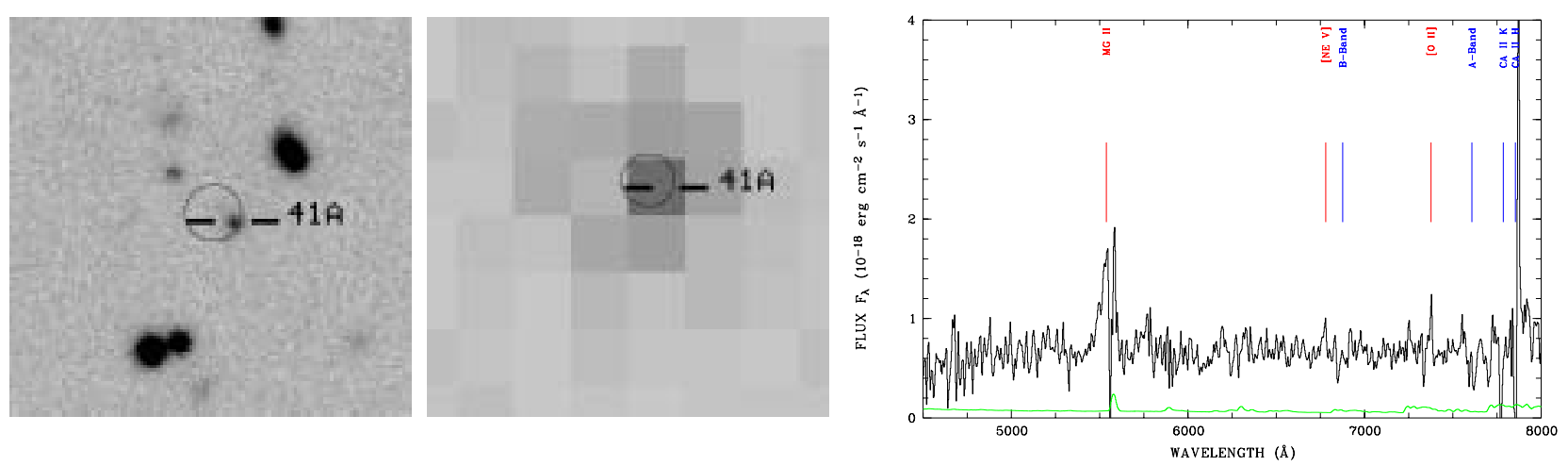

object $41 \mathrm{~A}, \mathrm{z}=0.979$
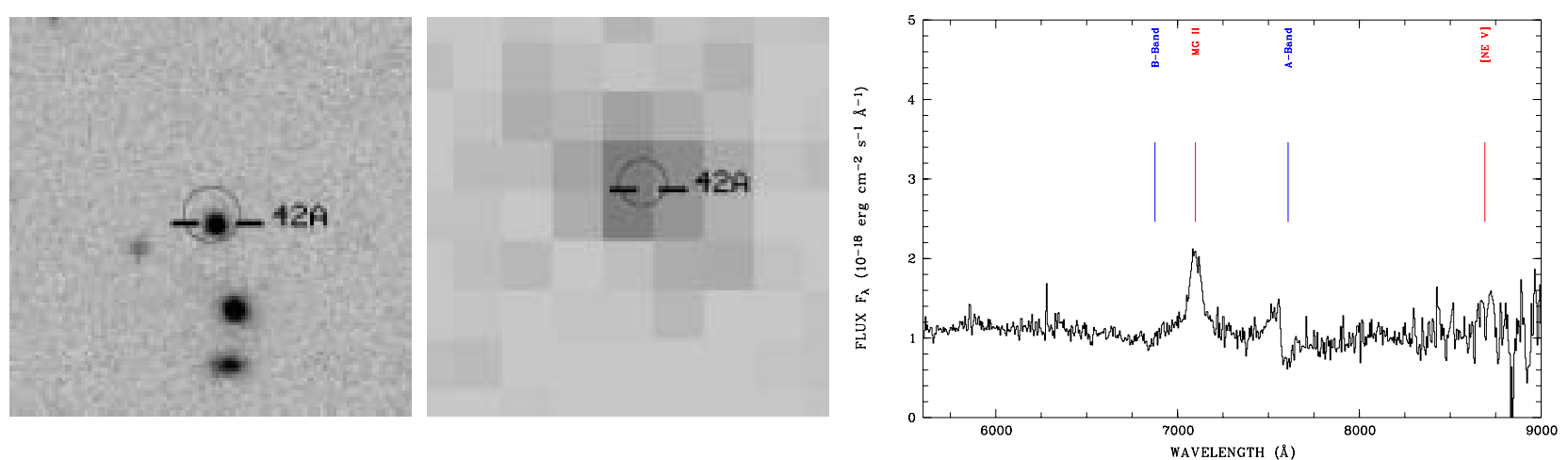

object $42 \mathrm{~A}, \mathrm{z}=1.54$
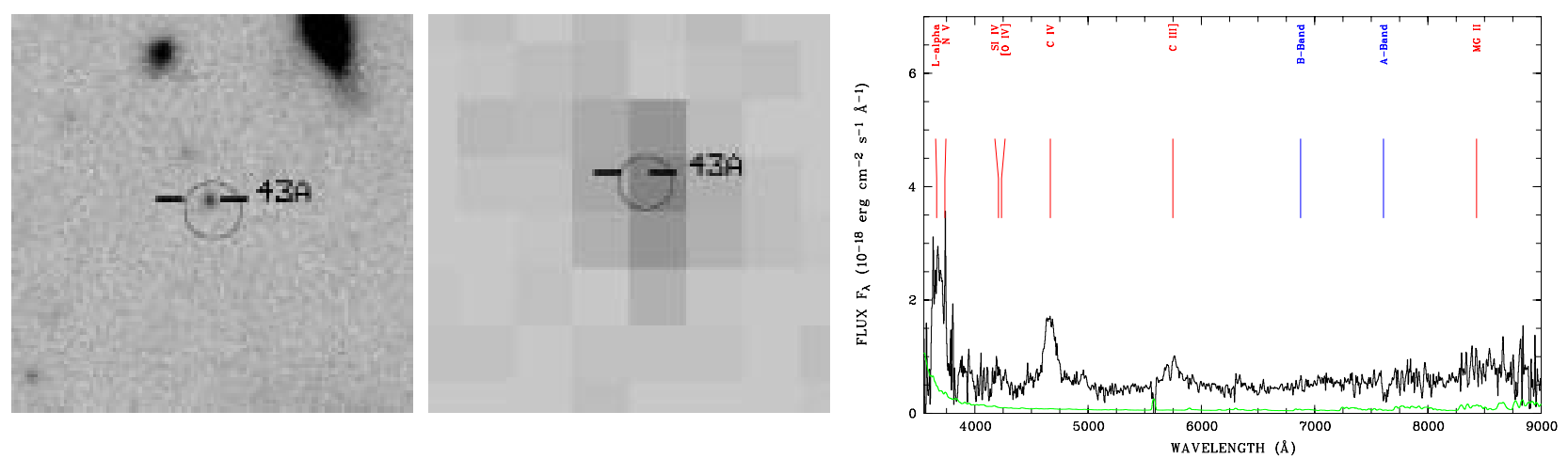

object $43 \mathrm{~A}, \mathrm{z}=2.013$
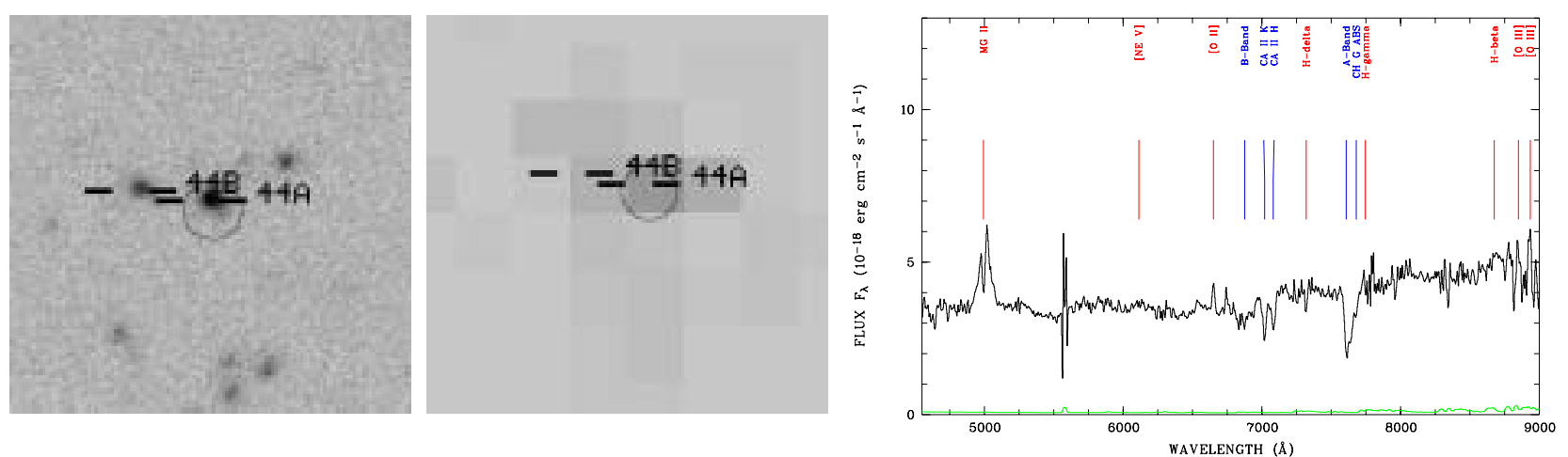

object $44 \mathrm{~A}, \mathrm{z}=0.785$ 
M. Krumpe et al.: The XMM-Newton survey in the Marano field. I., Online Material p 22
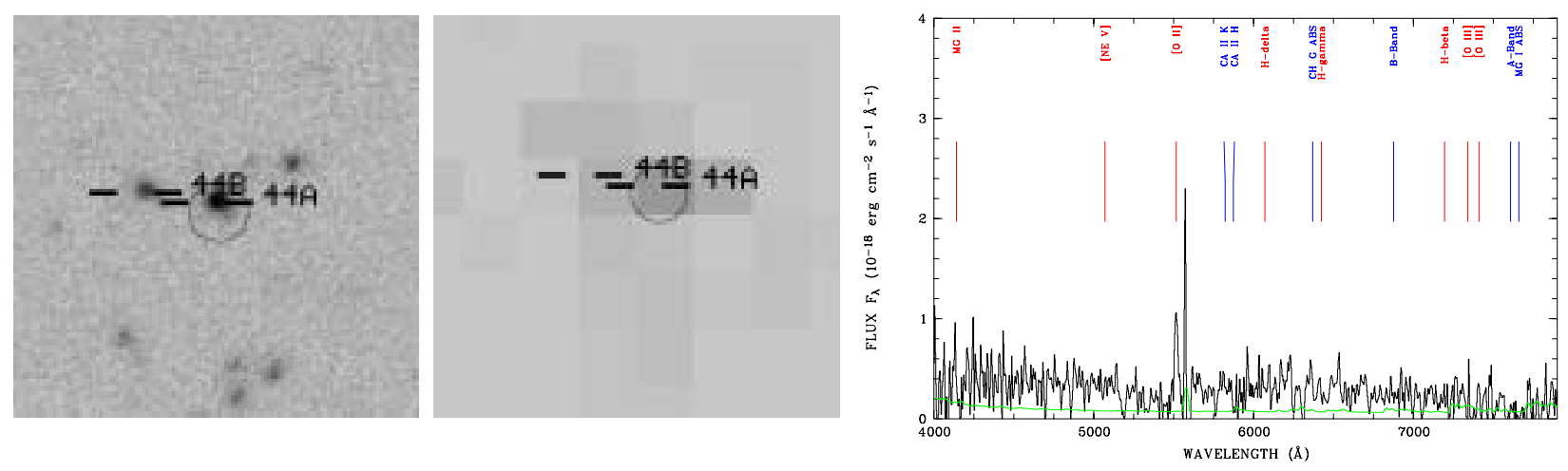

object 44B, z=0.480:@OII
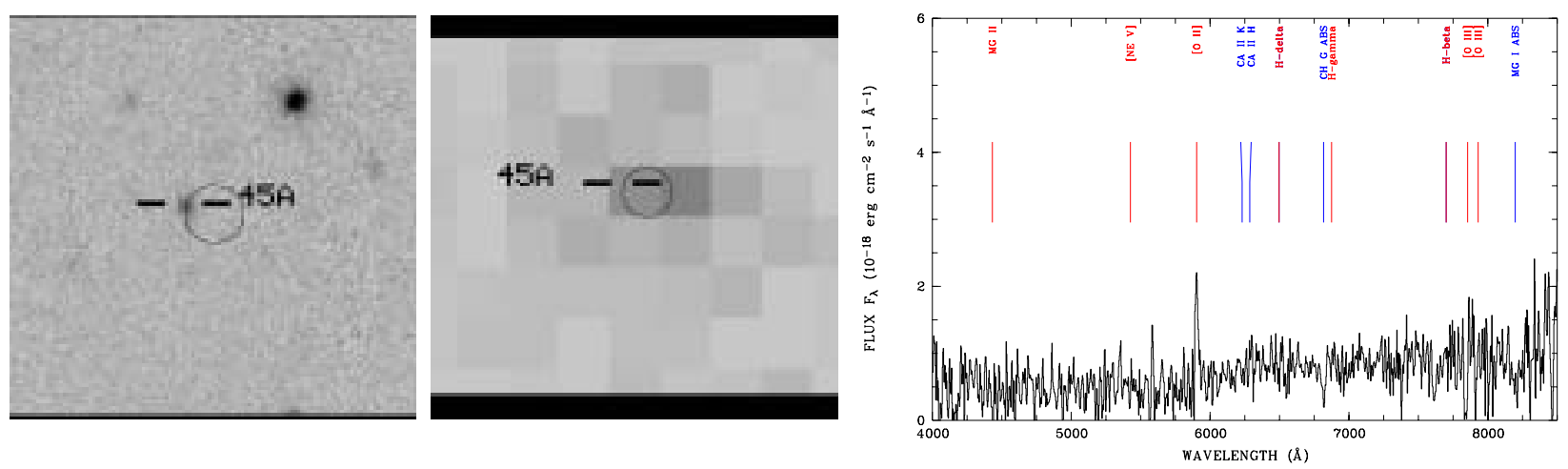

object 45A, z=0.584:@OII
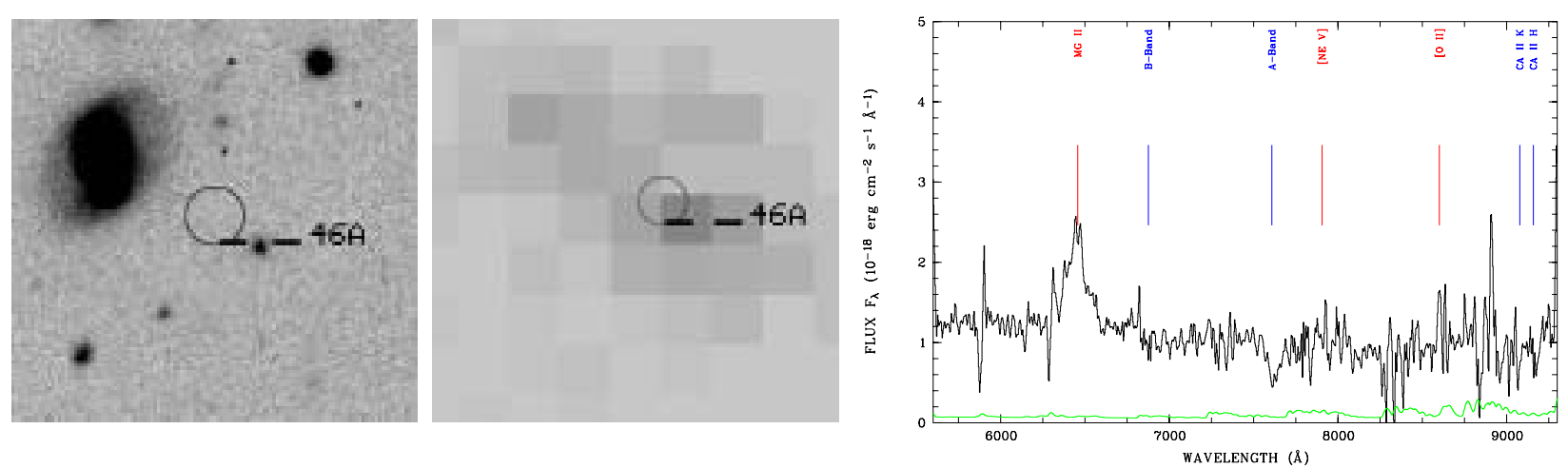

object $46 \mathrm{~A}, \mathrm{z}=1.308$
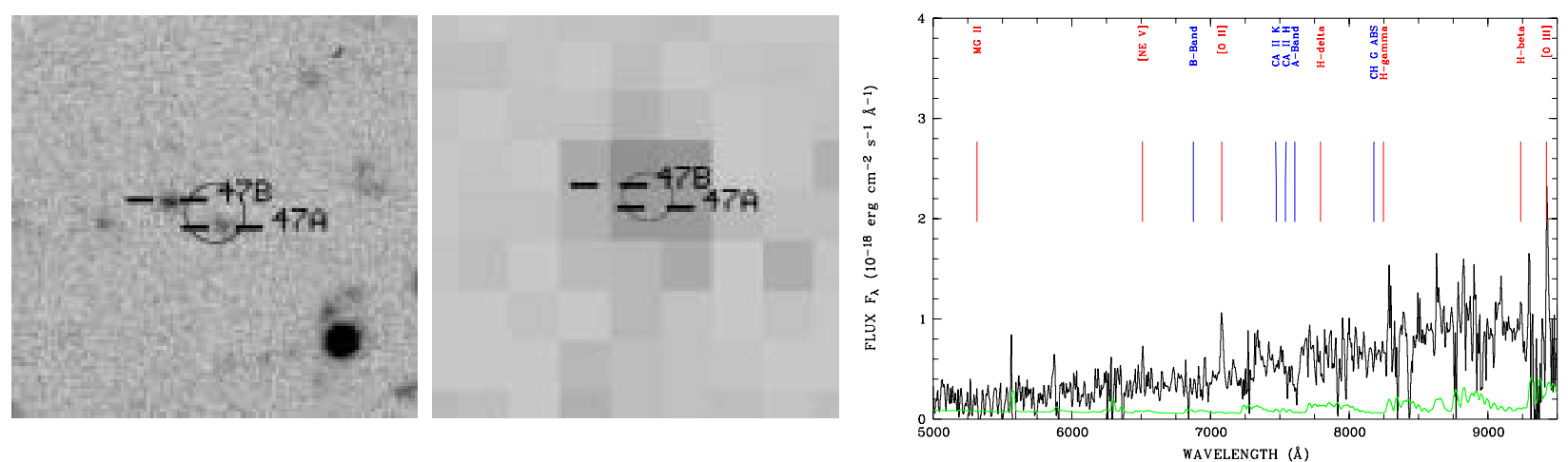

object 47A, z=0.900:@OII 
M. Krumpe et al.: The XMM-Newton survey in the Marano field. I., Online Material p 23
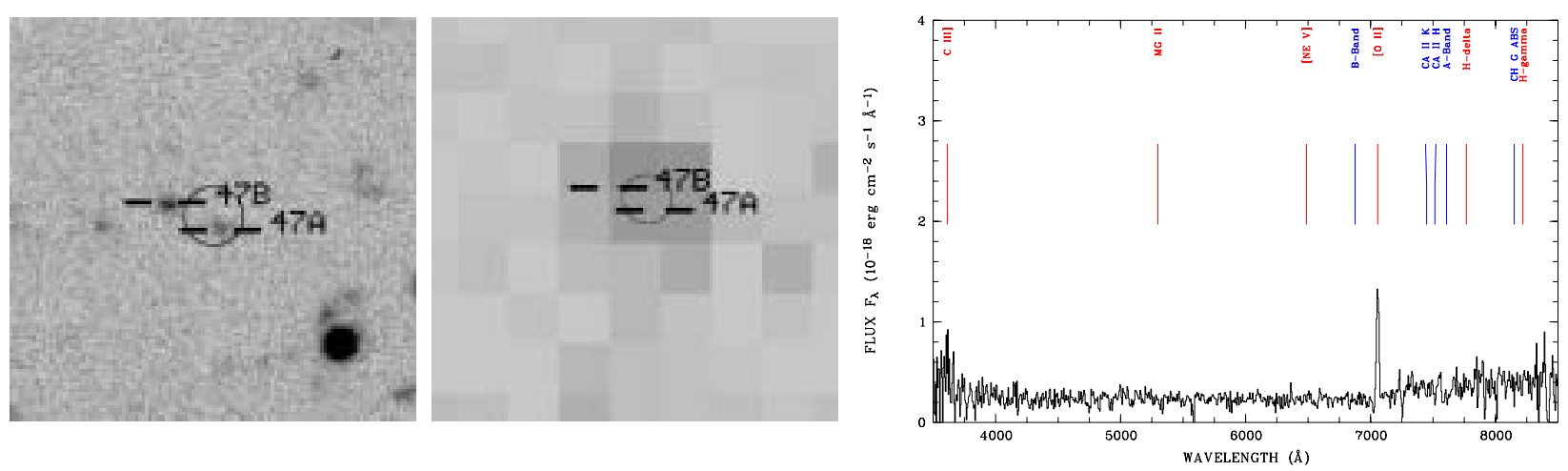

object 47B, z =0.89:@OII
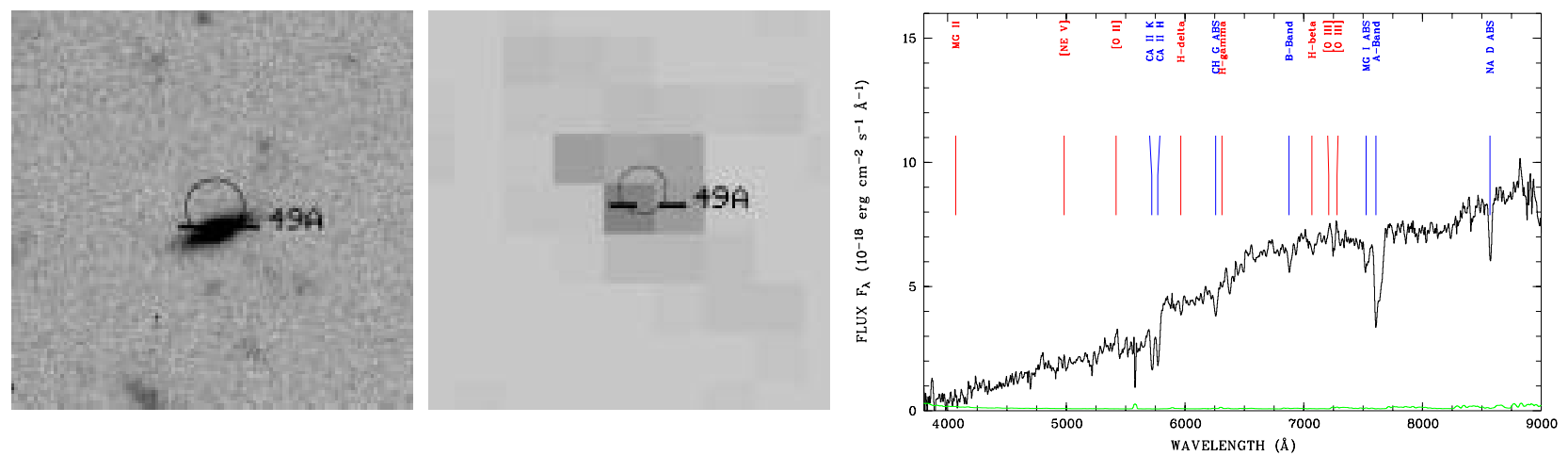

object $49 \mathrm{~A}, \mathrm{z}=0.454$
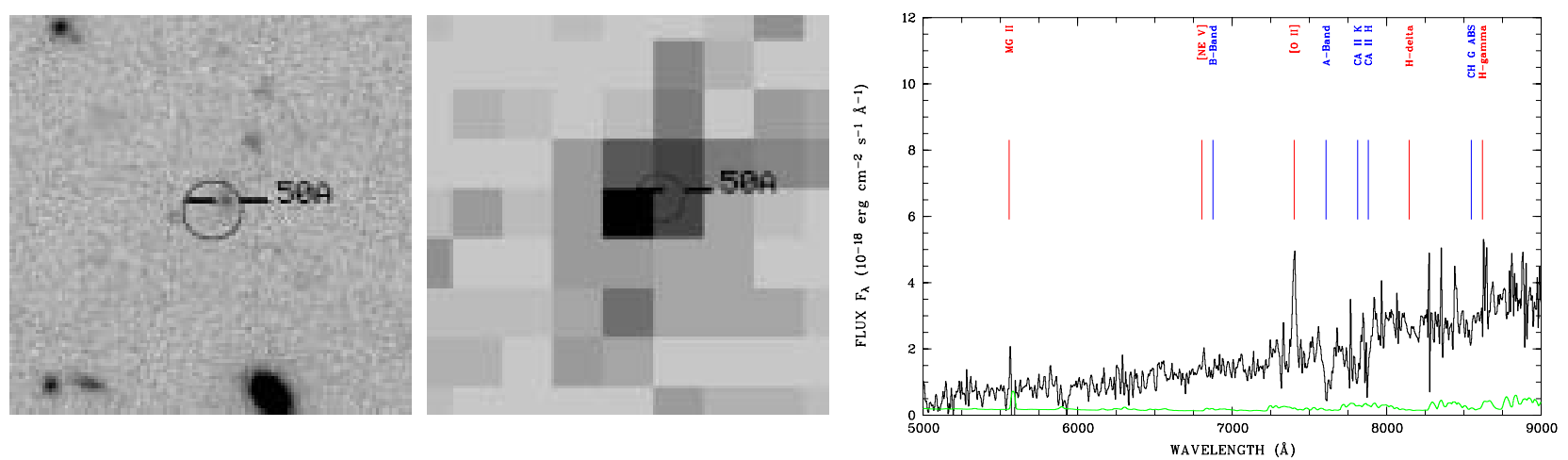

object 50A, z $=0.986$
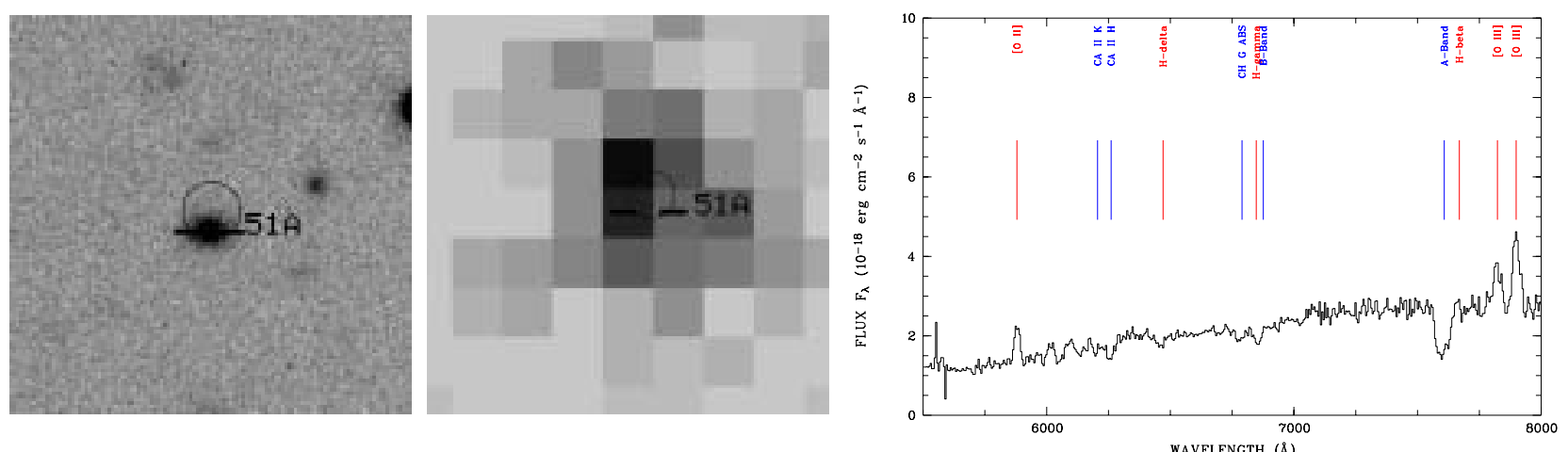

object $51 \mathrm{~A}, \mathrm{z}=0.58$ 
M. Krumpe et al.: The XMM-Newton survey in the Marano field. I., Online Material p 24
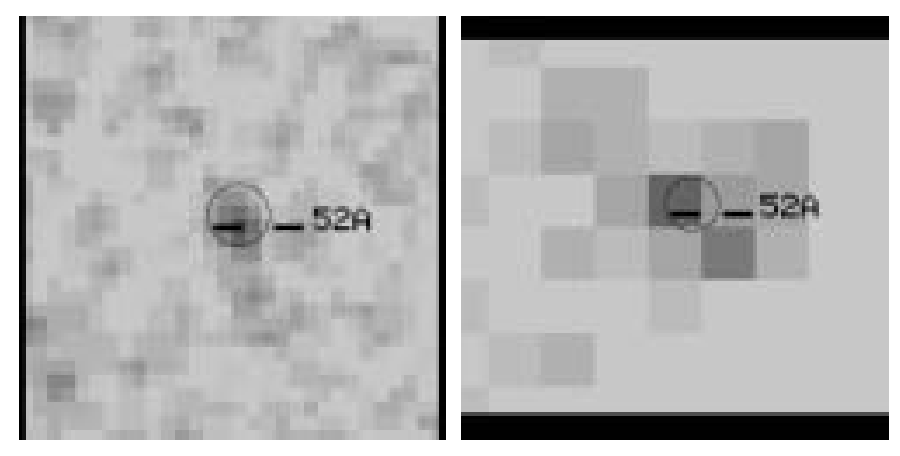

object $52 \mathrm{~A}, \mathrm{z}=1.829$
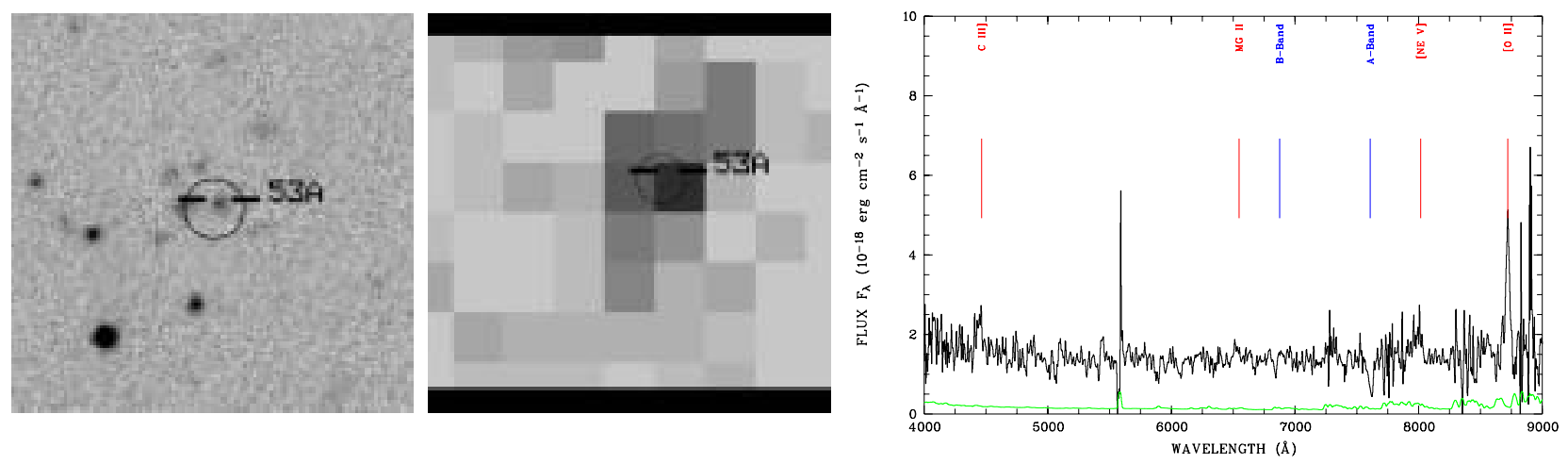

object 53A, z=1.340:@OII
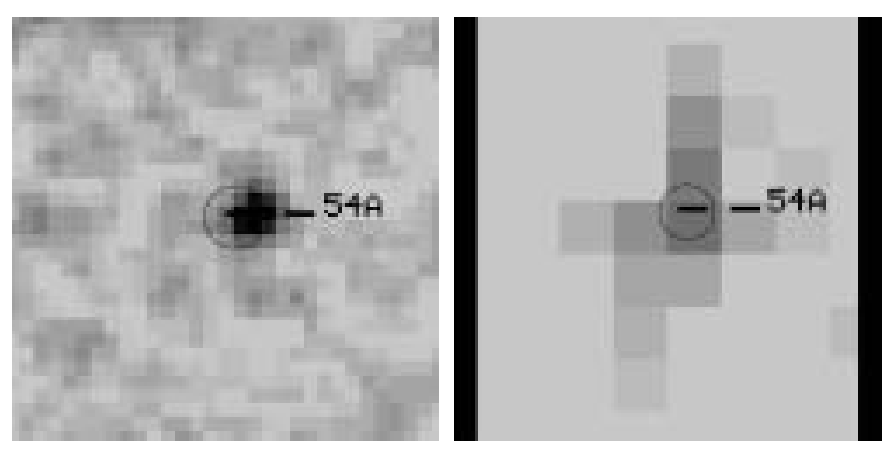

object $54 \mathrm{~A}, \mathrm{z}=2.744$
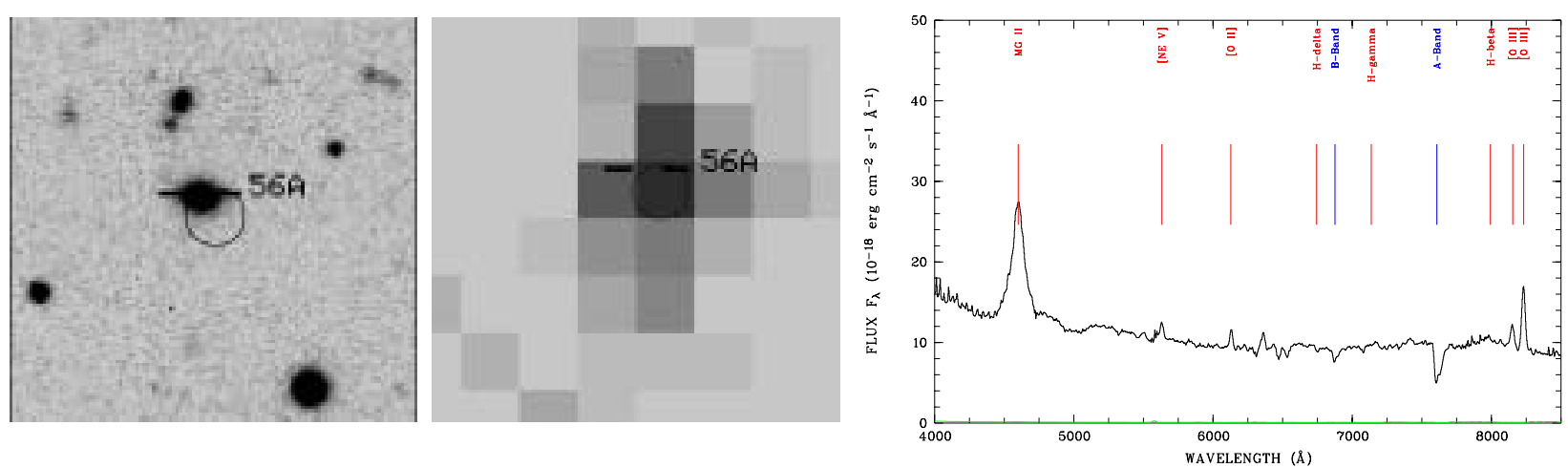

object $56 \mathrm{~A}, \mathrm{z}=0.641$ 
M. Krumpe et al.: The XMM-Newton survey in the Marano field. I., Online Material p 25
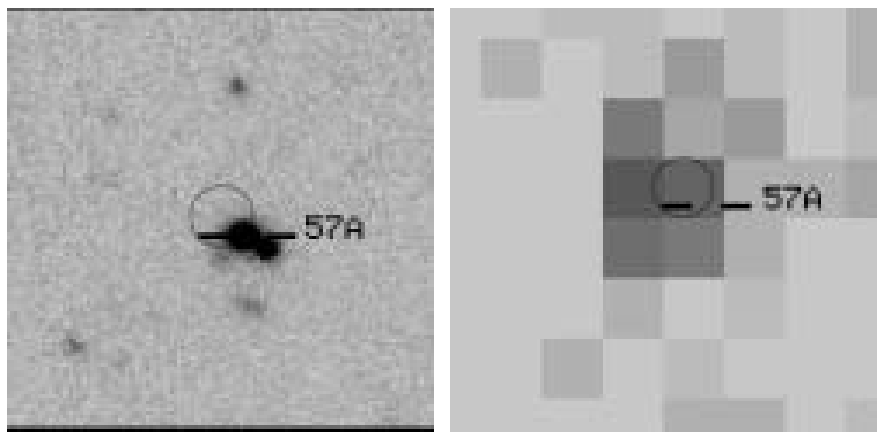

object $57 \mathrm{~A}, \mathrm{z}=0.281$
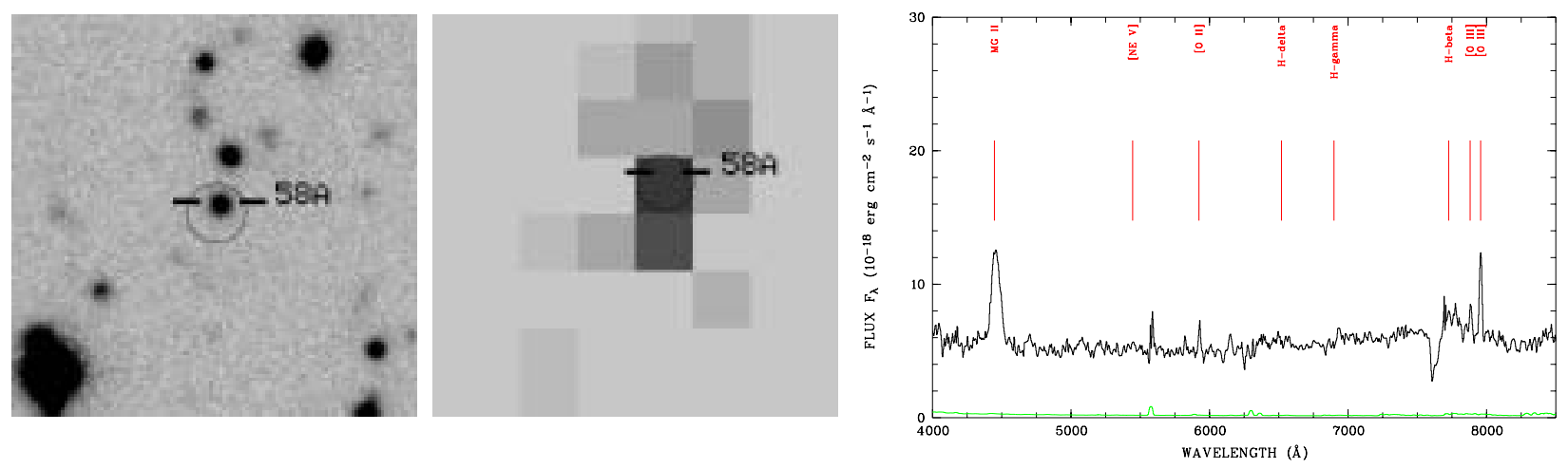

object $58 \mathrm{~A}, \mathrm{z}=0.589$
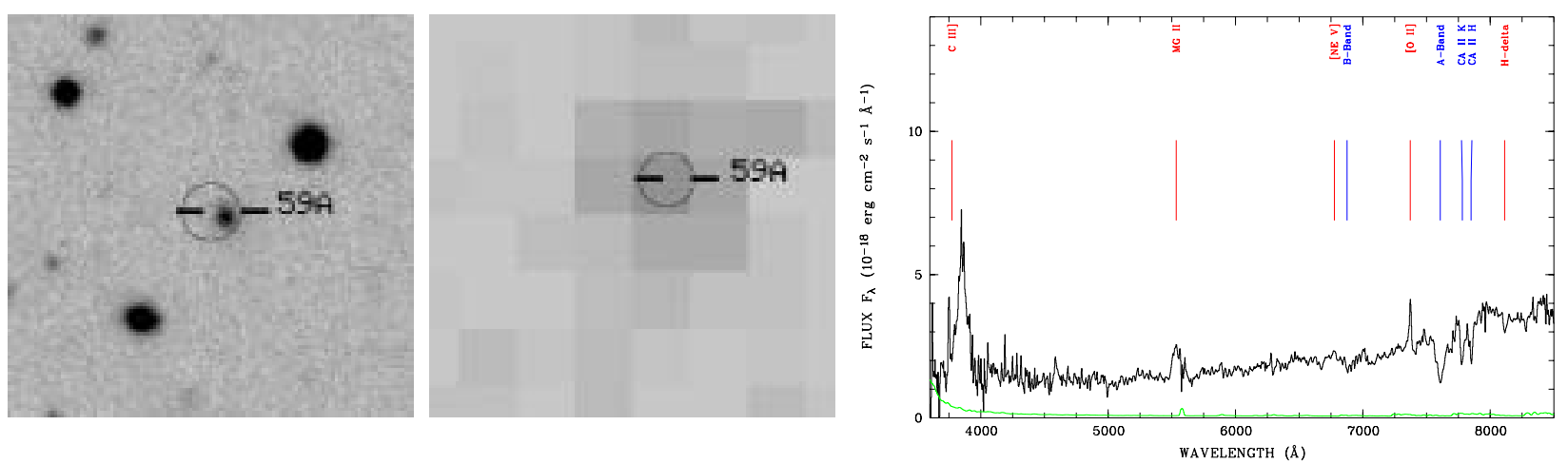

object 59A, $\mathrm{z}=0.978$
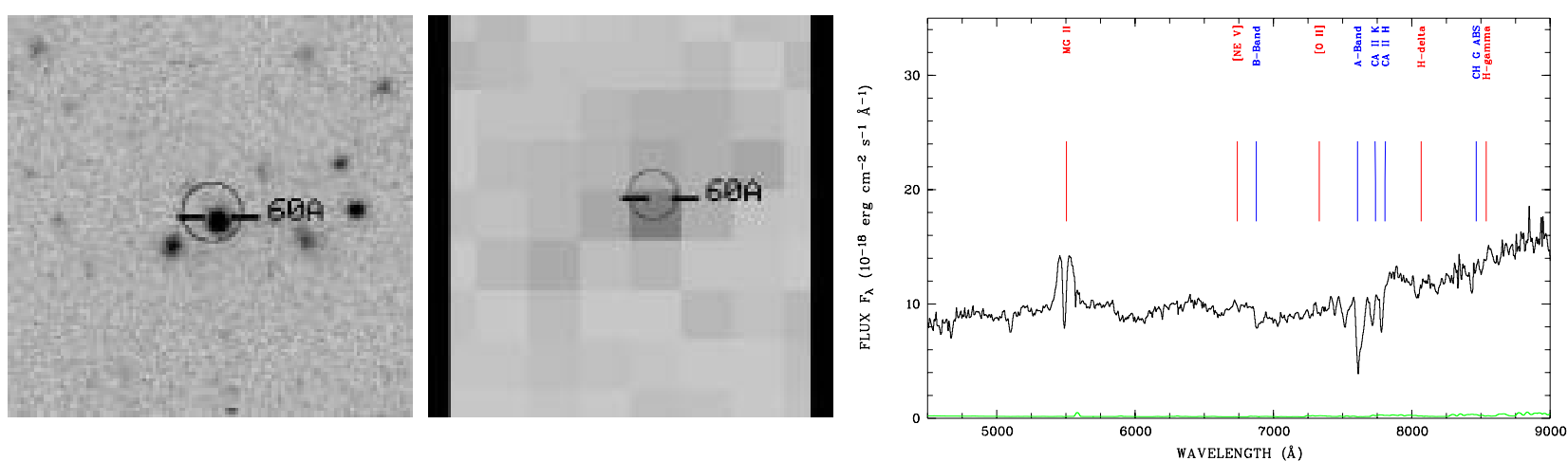

object $60 \mathrm{~A}, \mathrm{z}=0.957$ 
M. Krumpe et al.: The XMM-Newton survey in the Marano field. I., Online Material p 26
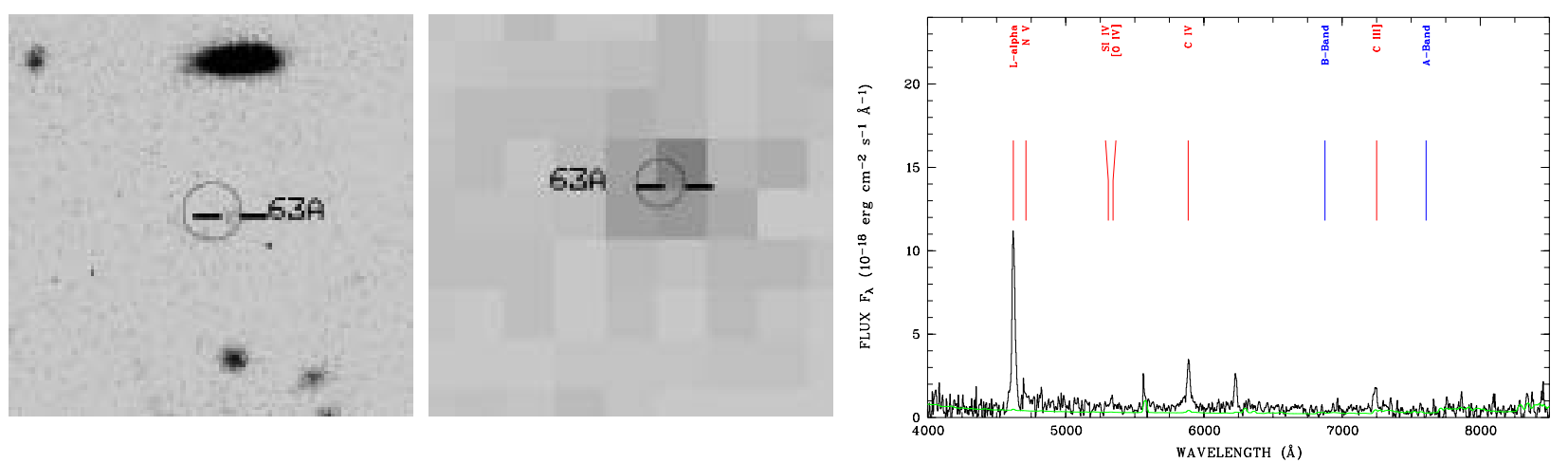

object $63 \mathrm{~A}, \mathrm{z}=2.800$
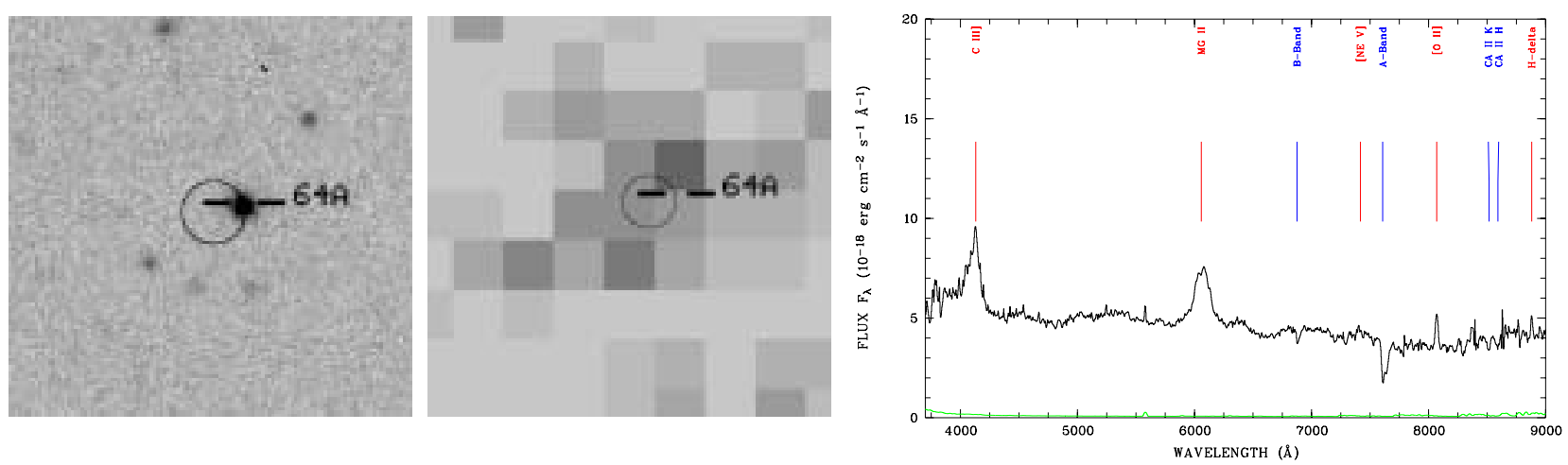

object $64 \mathrm{~A}, \mathrm{z}=1.165$
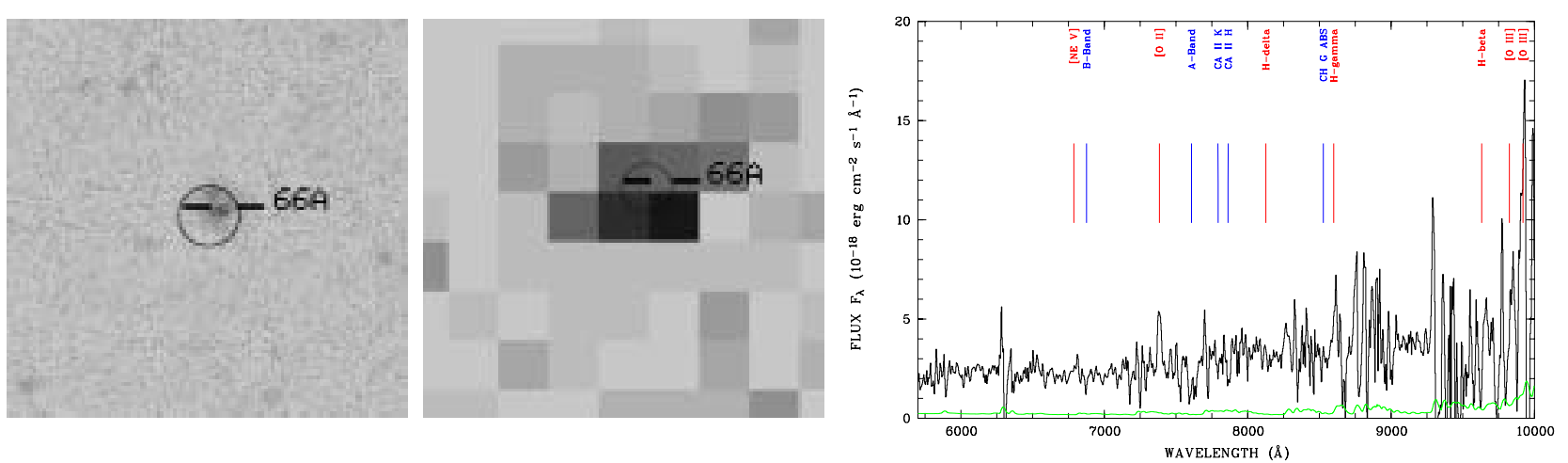

object 66A, z =0.981:@OII
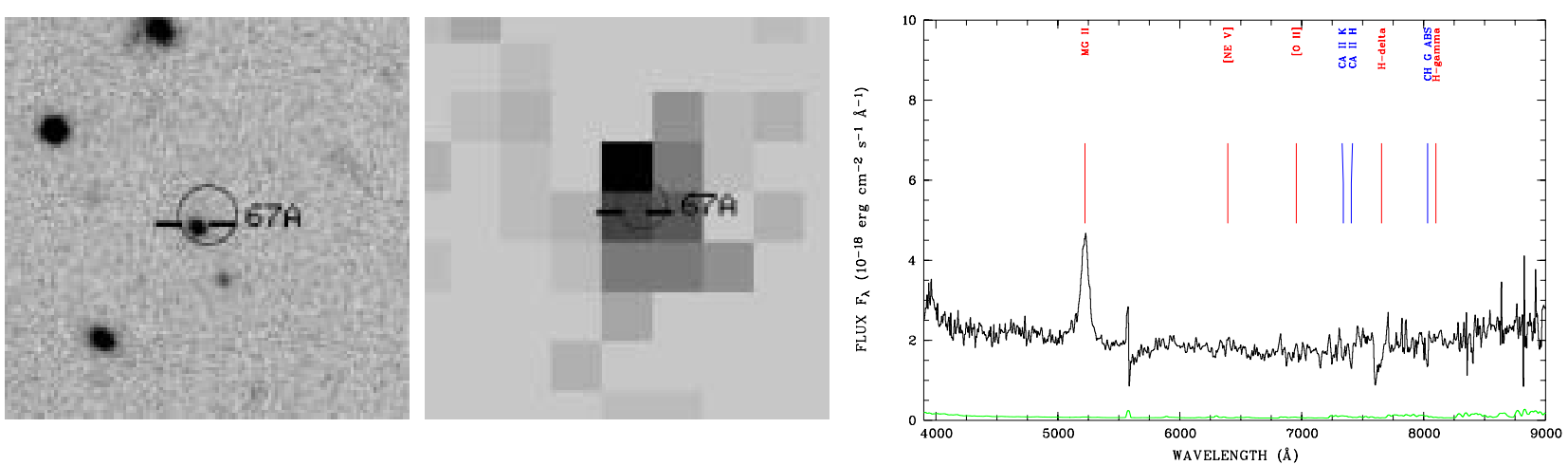

object $67 \mathrm{~A}, \mathrm{z}=0.866$ 
M. Krumpe et al.: The XMM-Newton survey in the Marano field. I., Online Material p 27
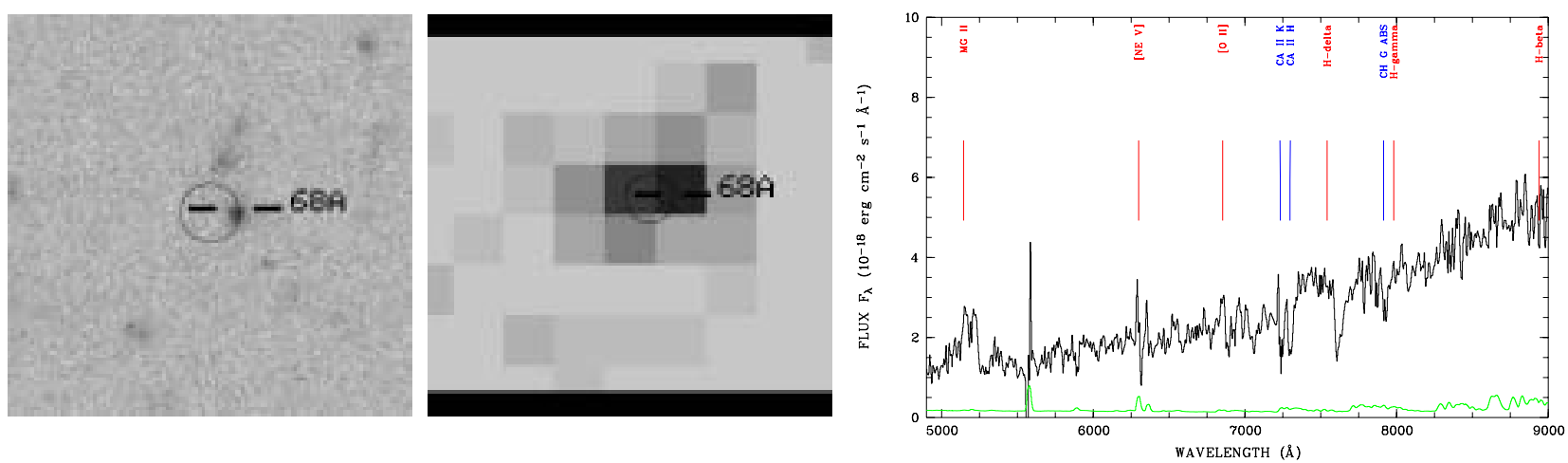

object $68 \mathrm{~A}, \mathrm{z}=0.839$
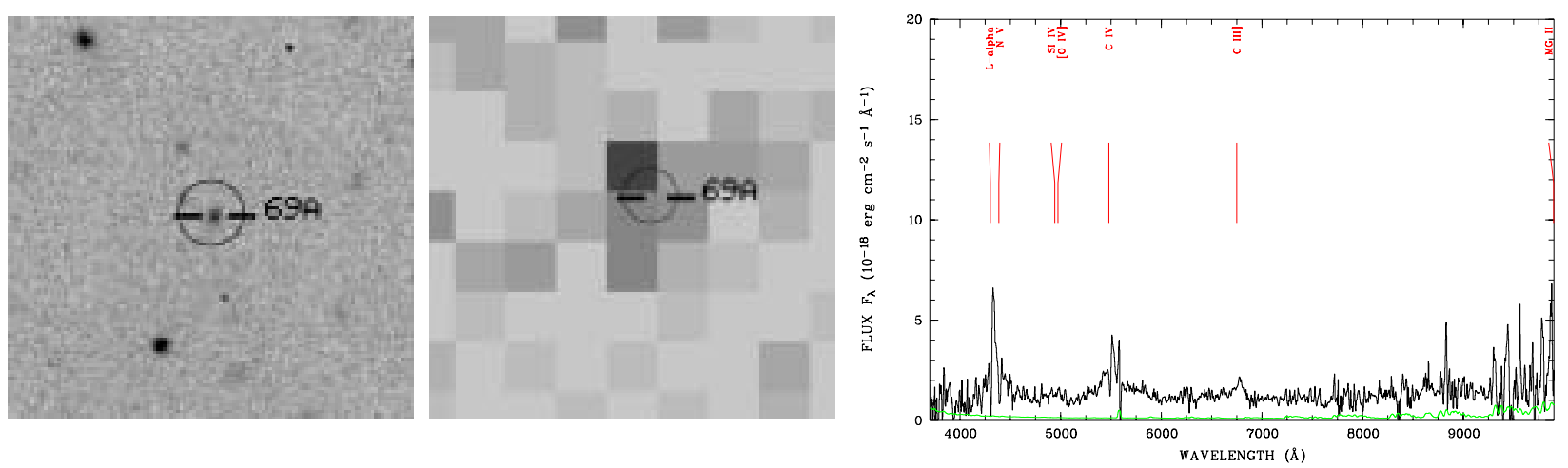

object $69 \mathrm{~A}, \mathrm{z}=2.553$
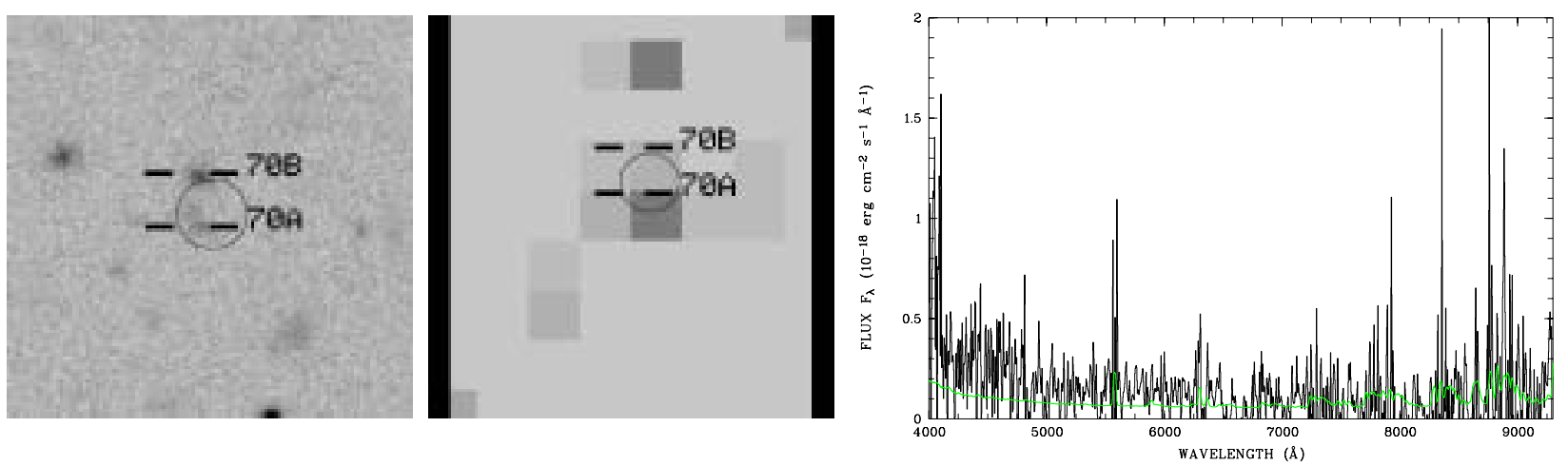

object 70A, no redshift, spectrum too noisy
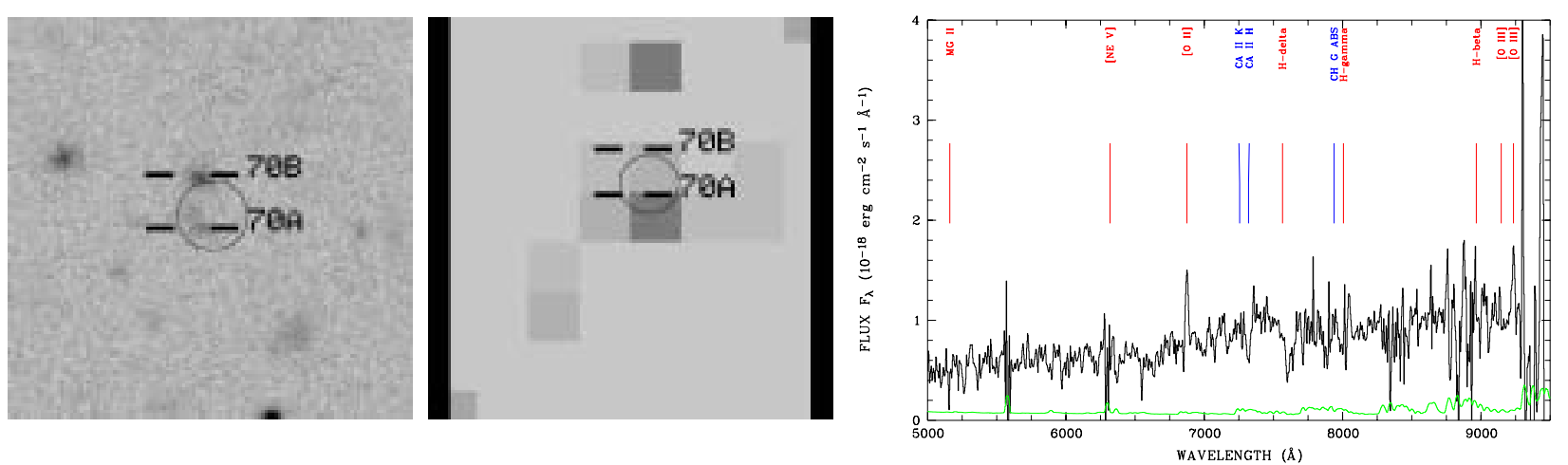

object $70 \mathrm{~B}, \mathrm{z}=0.844$ 

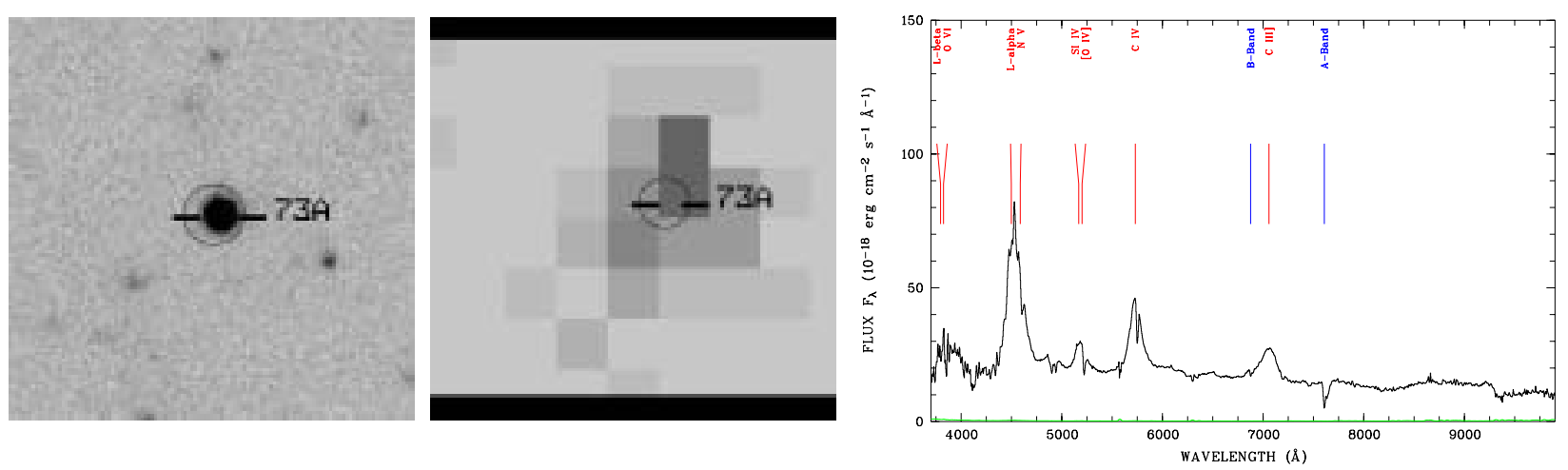

object $73 \mathrm{~A}, \mathrm{z}=2.706$
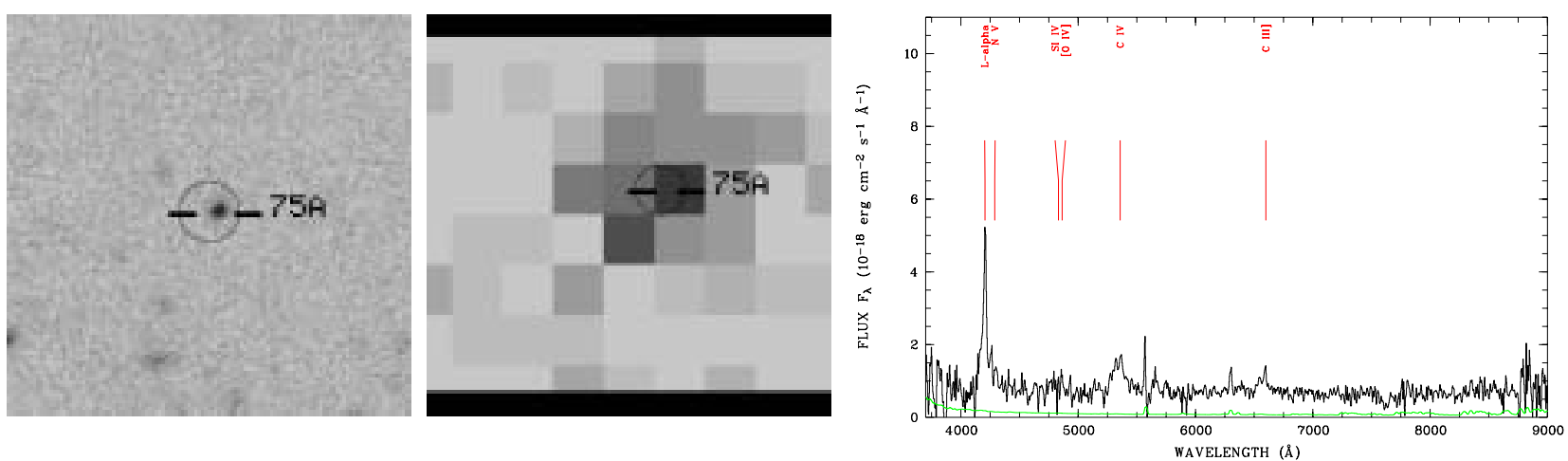

object $75 \mathrm{~A}, \mathrm{z}=2.460$
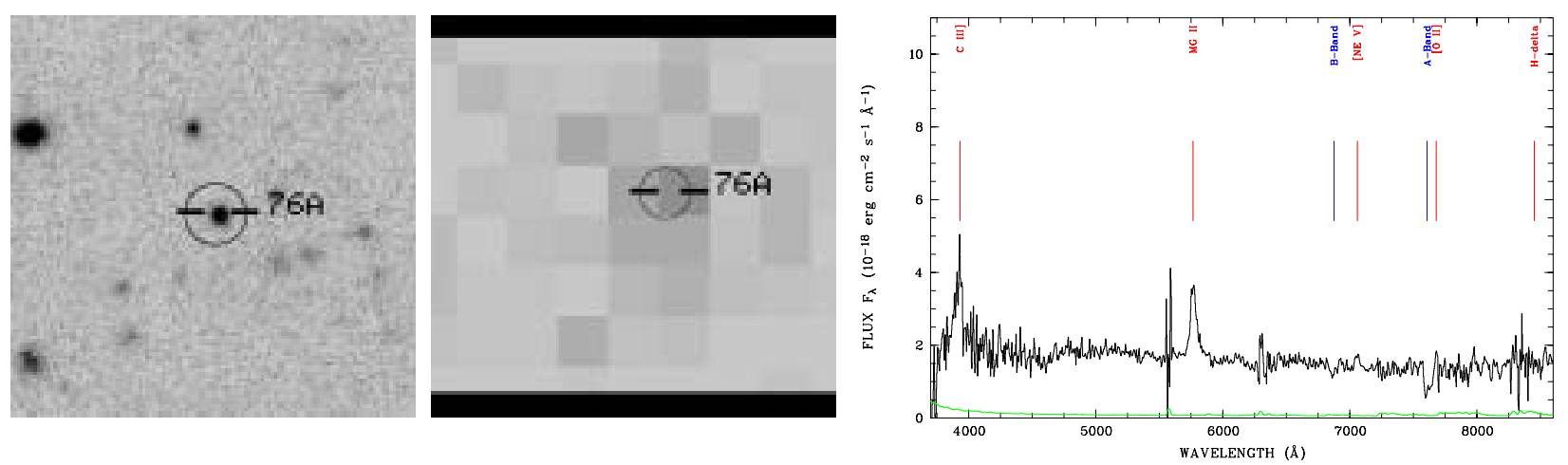

object 76A, $\mathrm{z}=1.065$
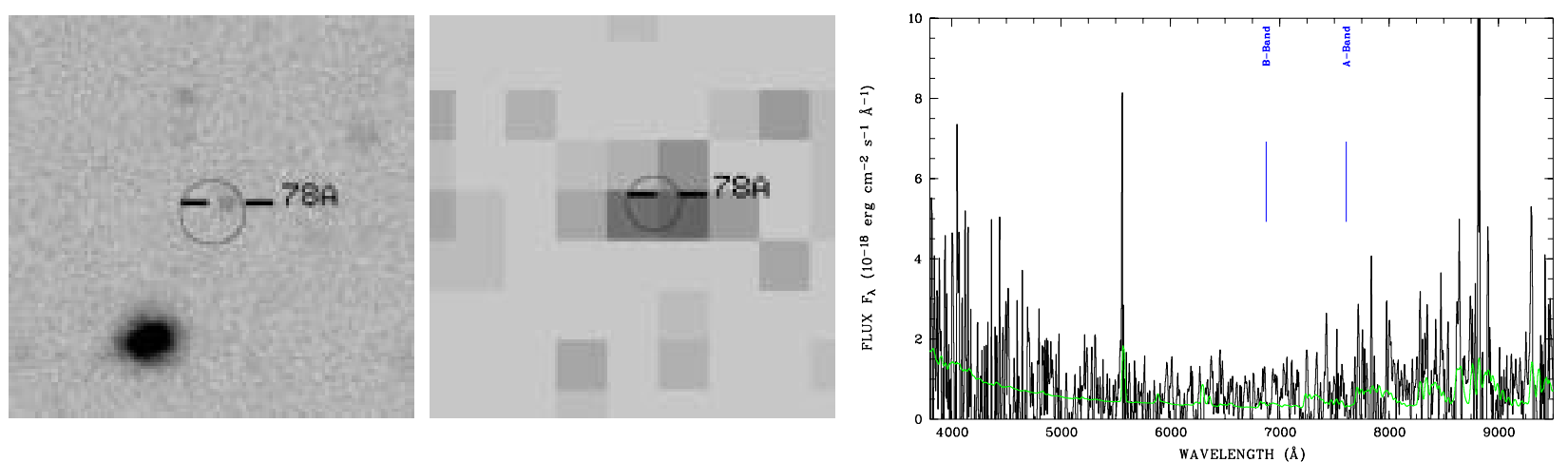

object 78A, no redshift, spectrum too noisy 
M. Krumpe et al.: The XMM-Newton survey in the Marano field. I., Online Material p 29
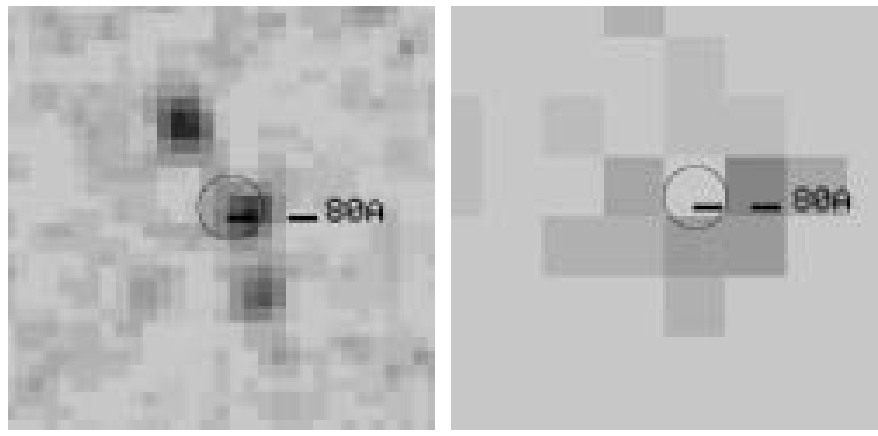

object $80 \mathrm{~A}, \mathrm{z}=1.620$
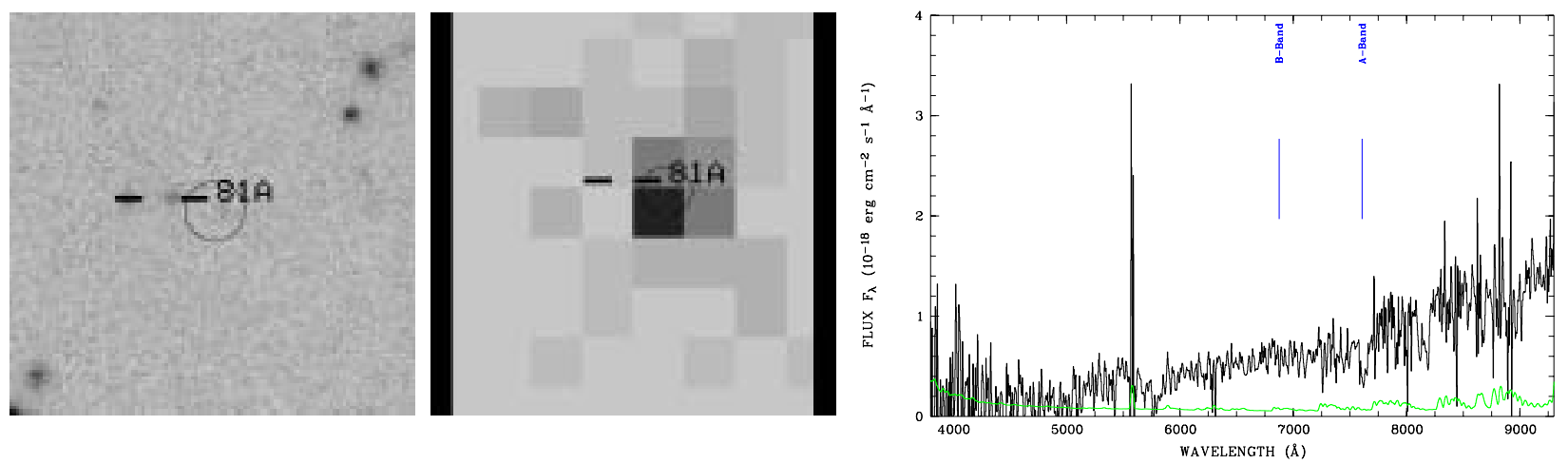

object $81 \mathrm{~A}$, no redshift, spectrum too noisy
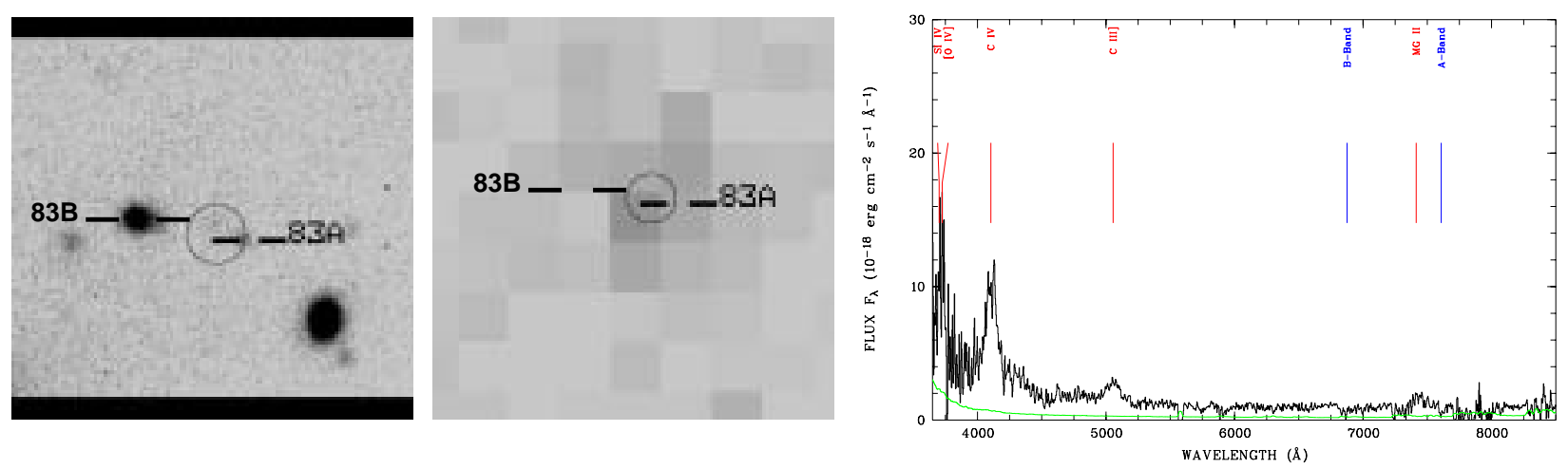

object $83 \mathrm{~A}, \mathrm{z}=1.650$
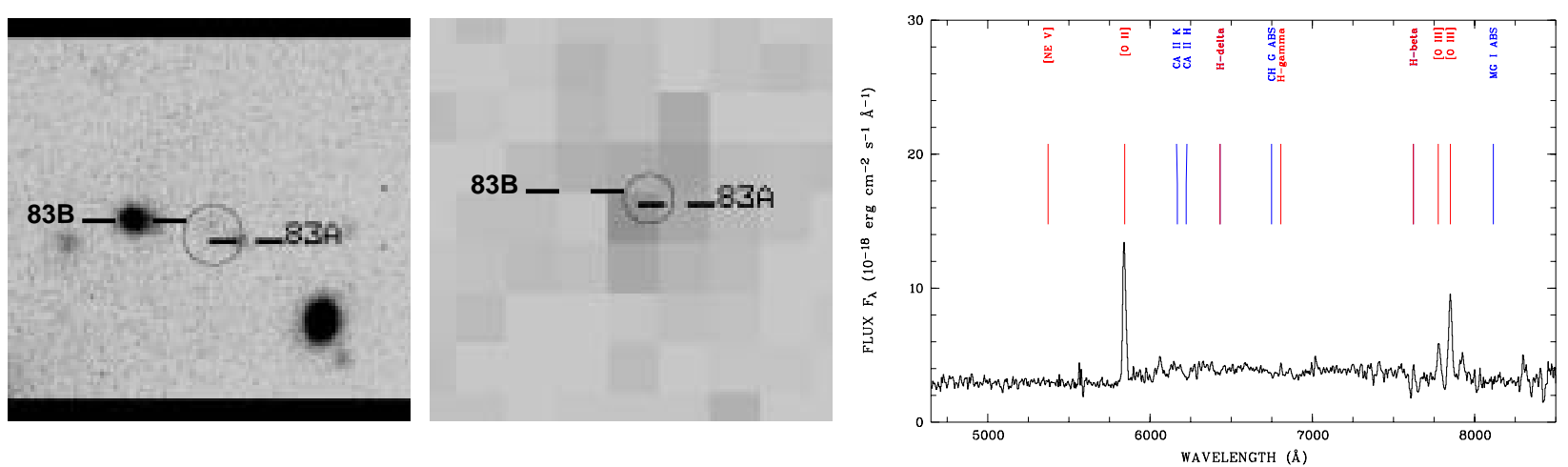

object $83 \mathrm{~B}, \mathrm{z}=0.568$ 
M. Krumpe et al.: The XMM-Newton survey in the Marano field. I., Online Material p 30
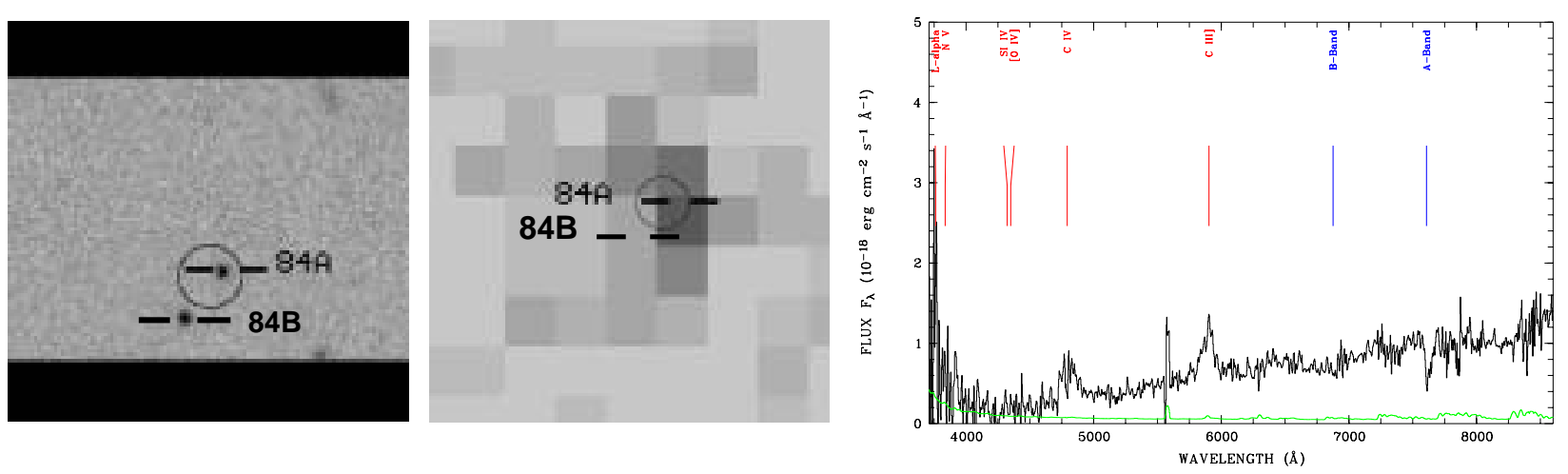

object $84 \mathrm{~A}, \mathrm{z}=2.094$
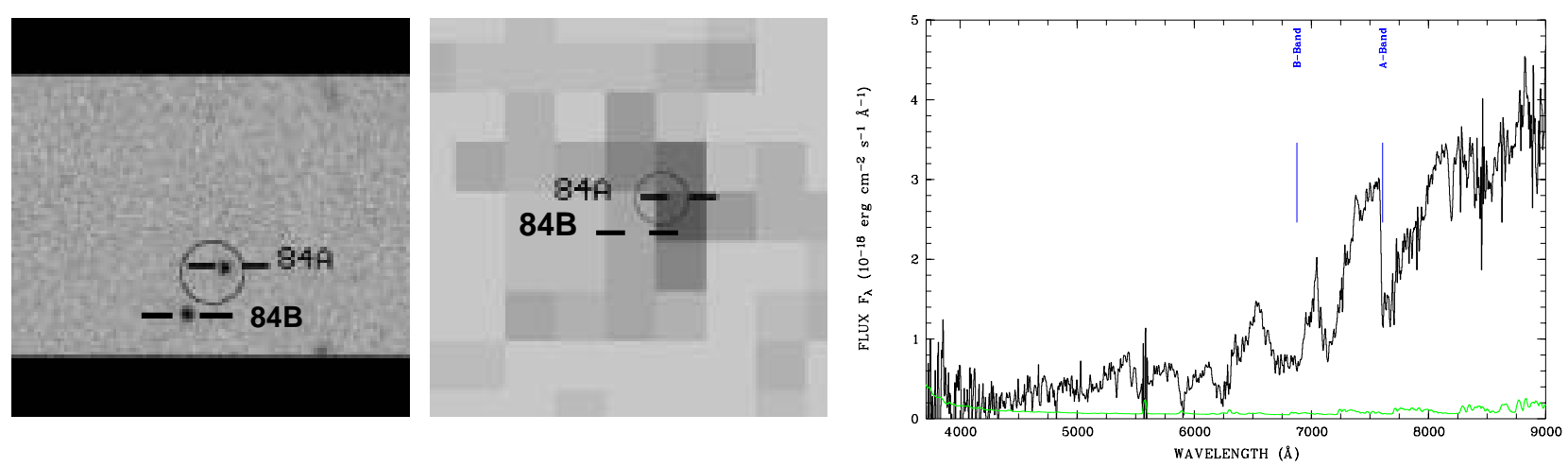

object $84 \mathrm{~B}, \mathrm{z}=0.000$
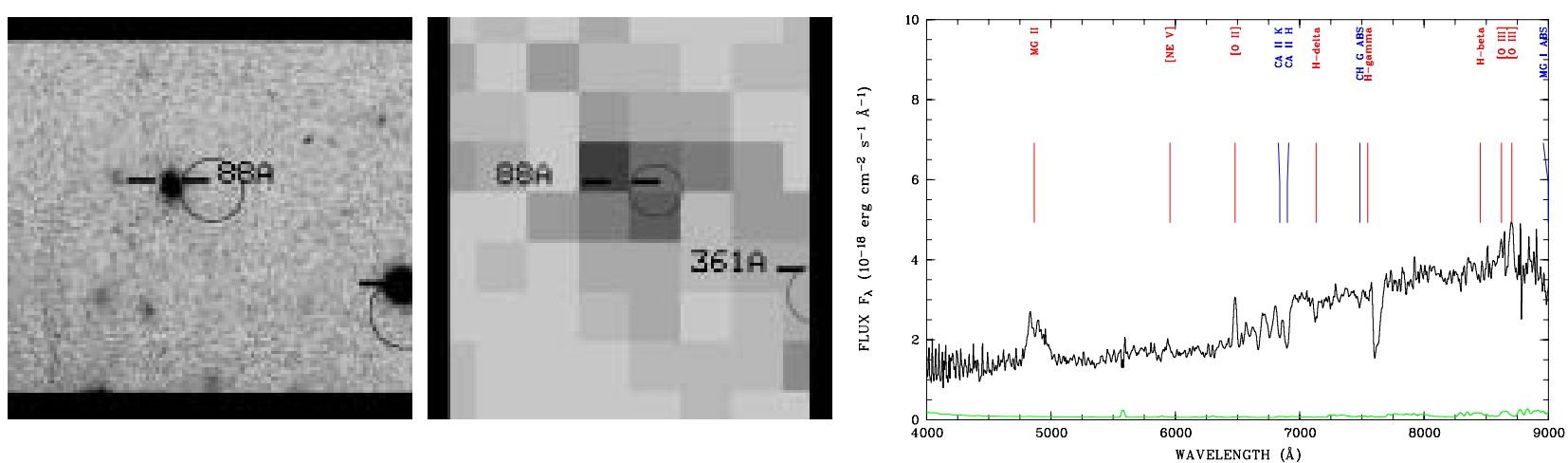

object $88 \mathrm{~A}, \mathrm{z}=0.739$
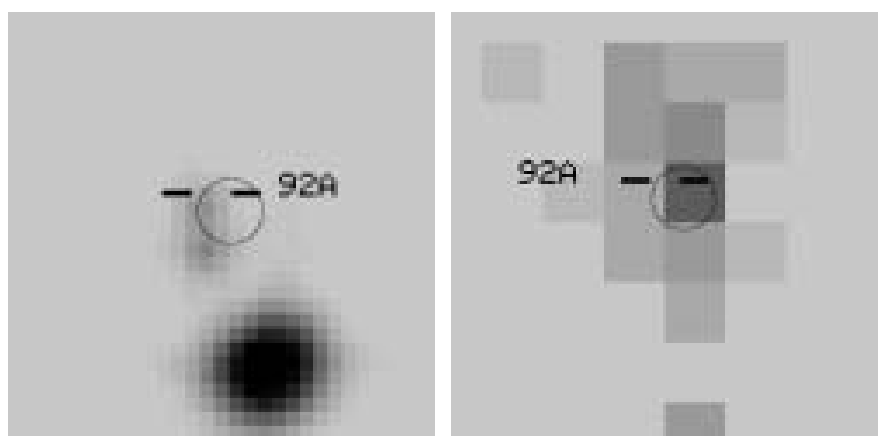

object $92 \mathrm{~A}, \mathrm{z}=2.425$ 
M. Krumpe et al.: The XMM-Newton survey in the Marano field. I., Online Material p 31
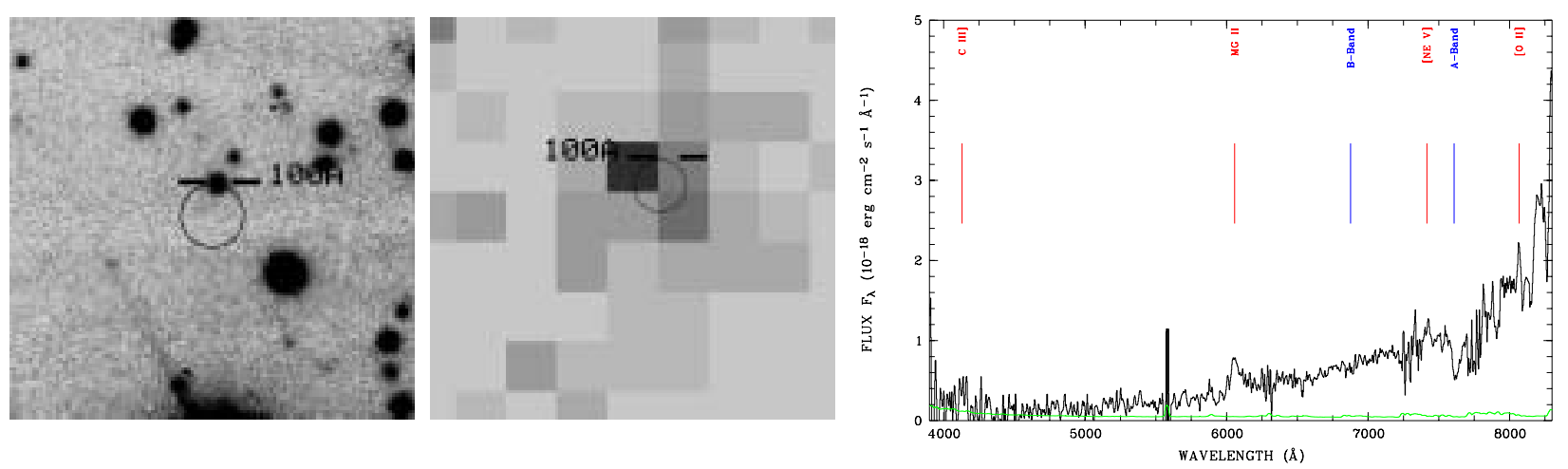

object 100A, $\mathrm{z}=1.165$
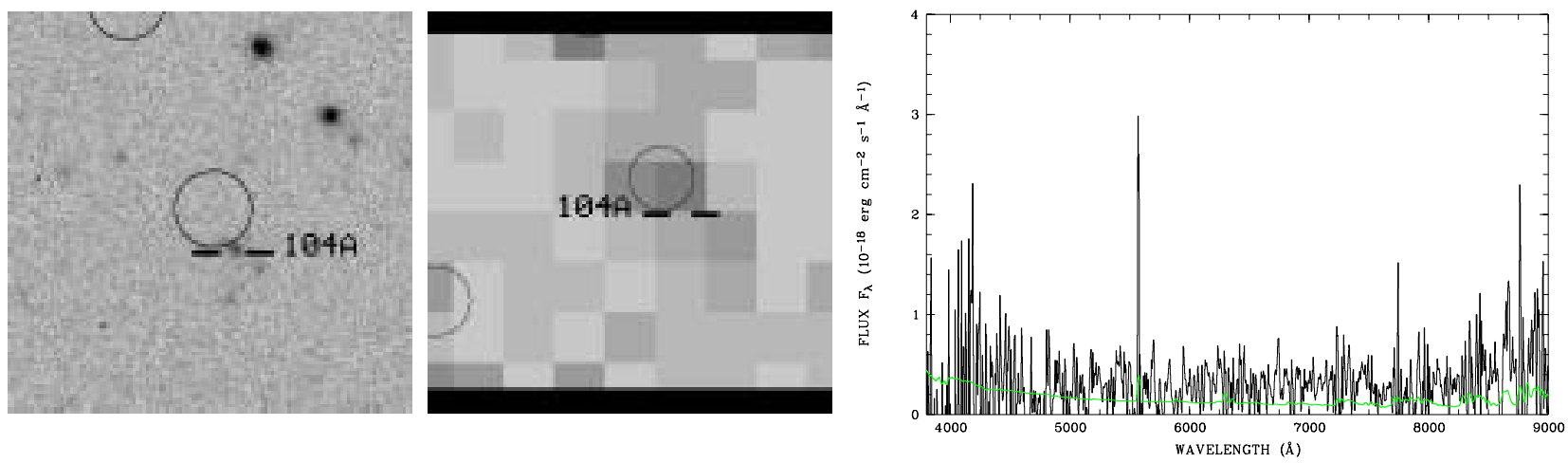

object 104A, no redshift, spectrum too noisy
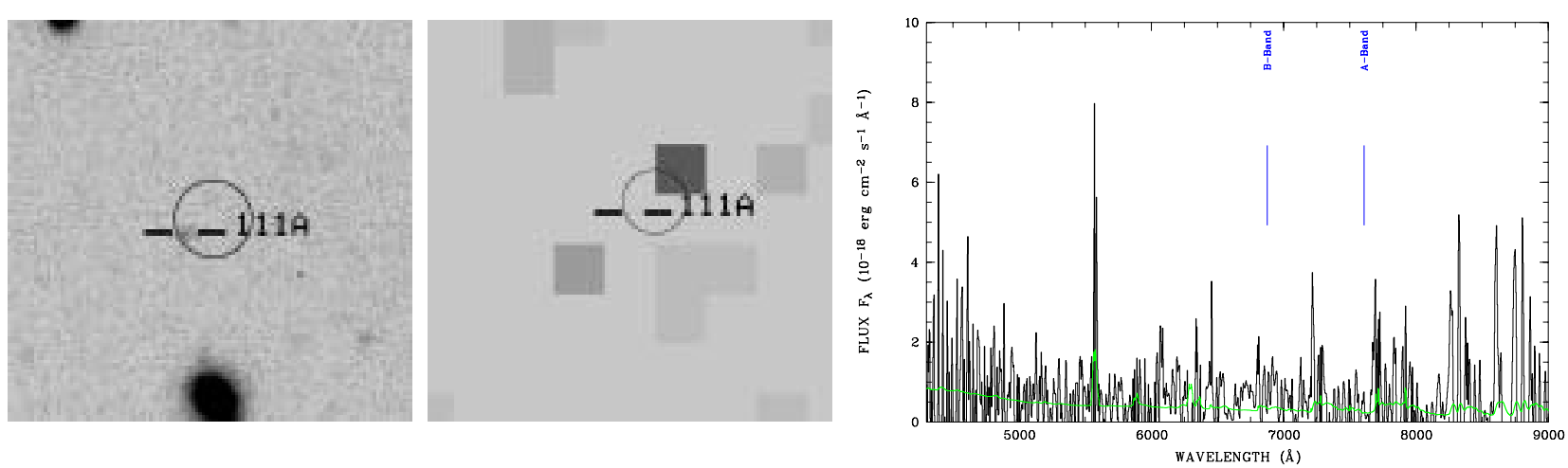

object 111A, no redshift, spectrum too noisy
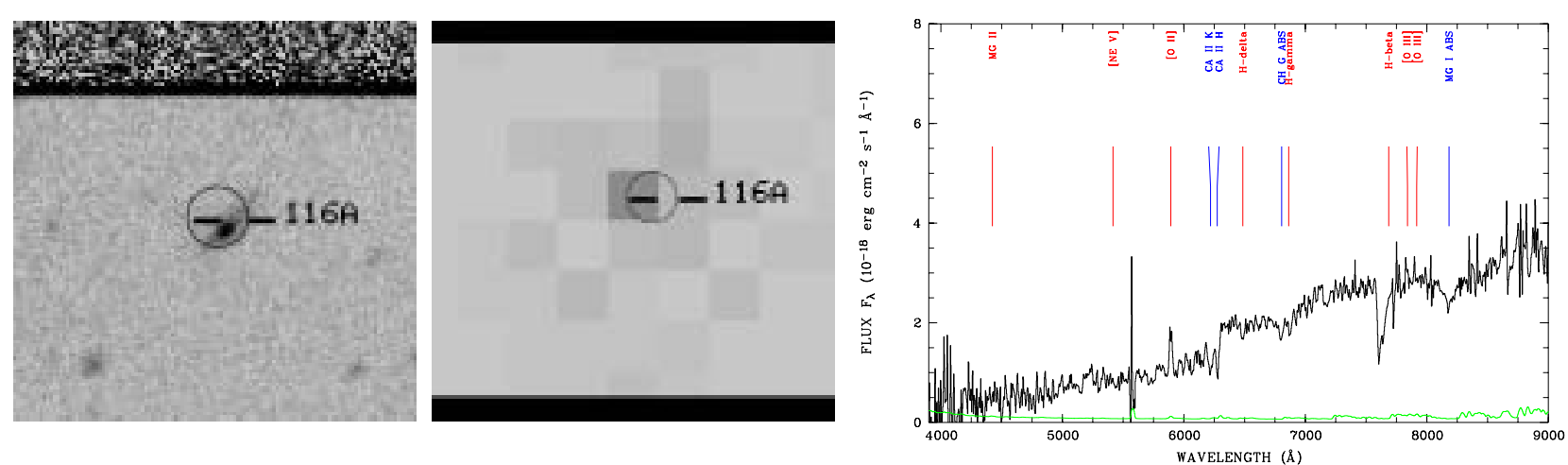

object 116A, $\mathrm{z}=0.581$ 


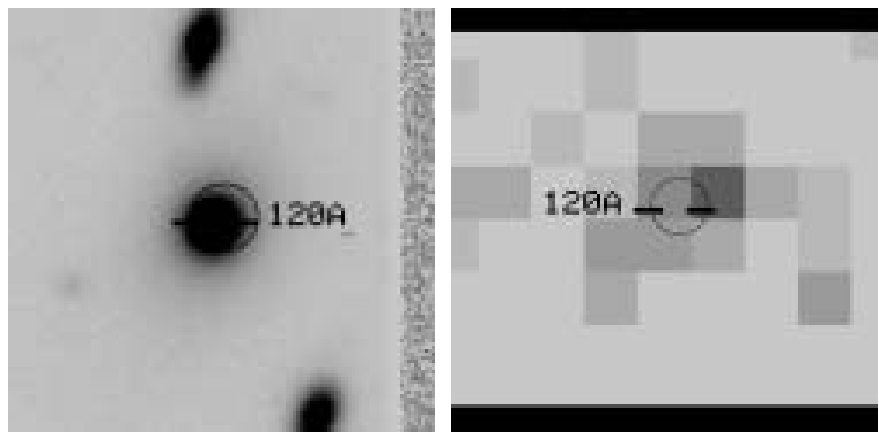

object $120 \mathrm{~A}, \mathrm{z}=0.094$
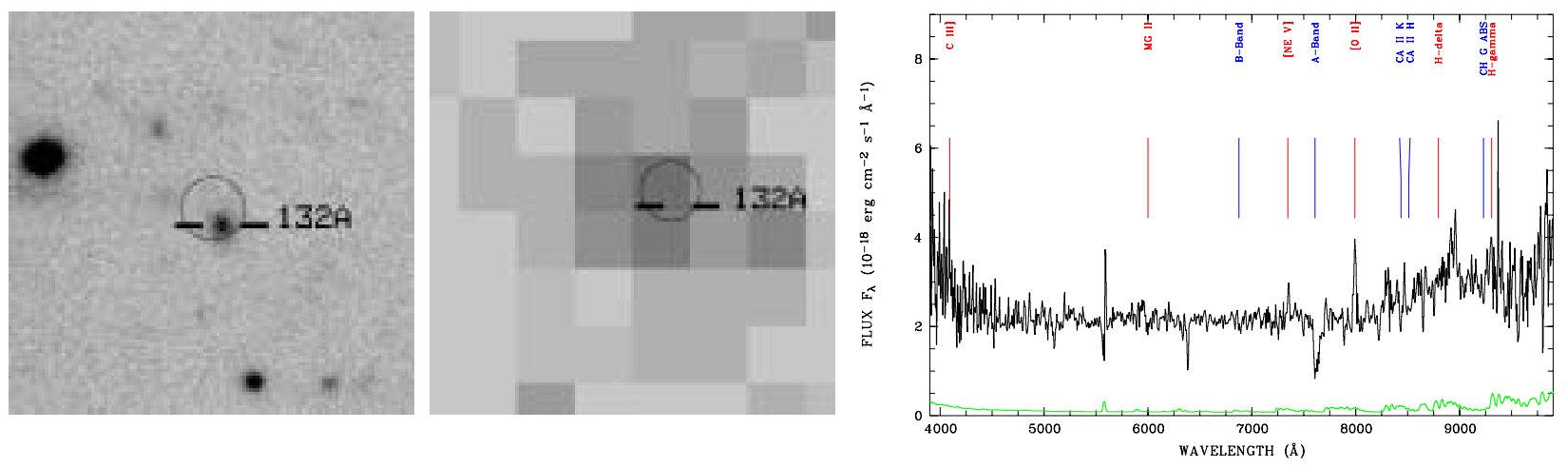

object 132A, z=1.144:@OII
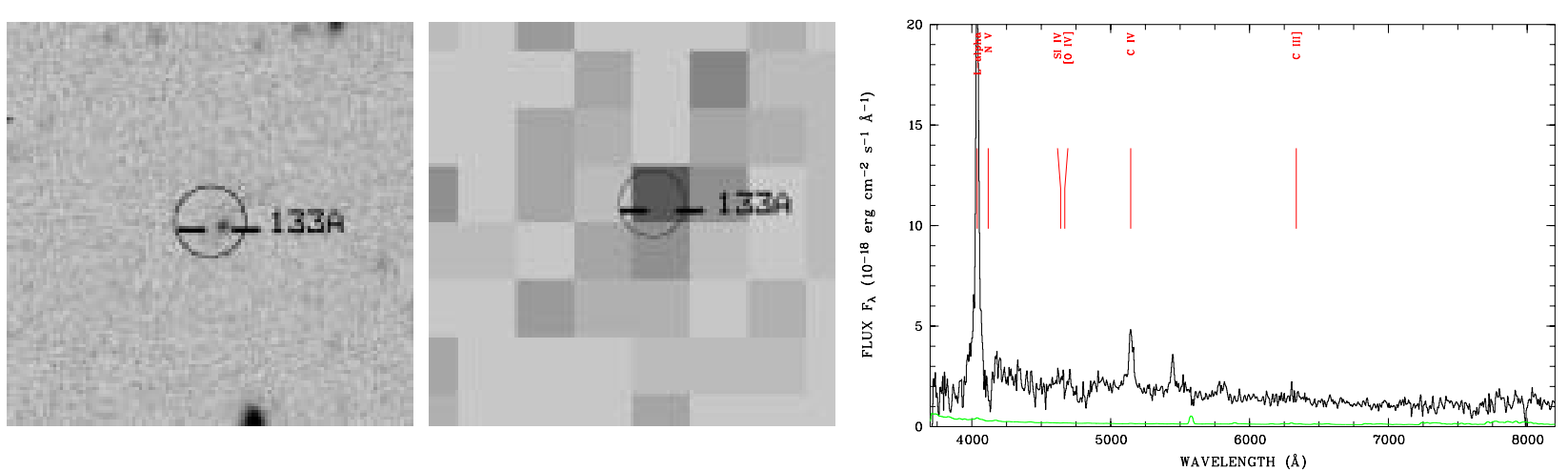

object $133 \mathrm{~A}, \mathrm{z}=2.321$
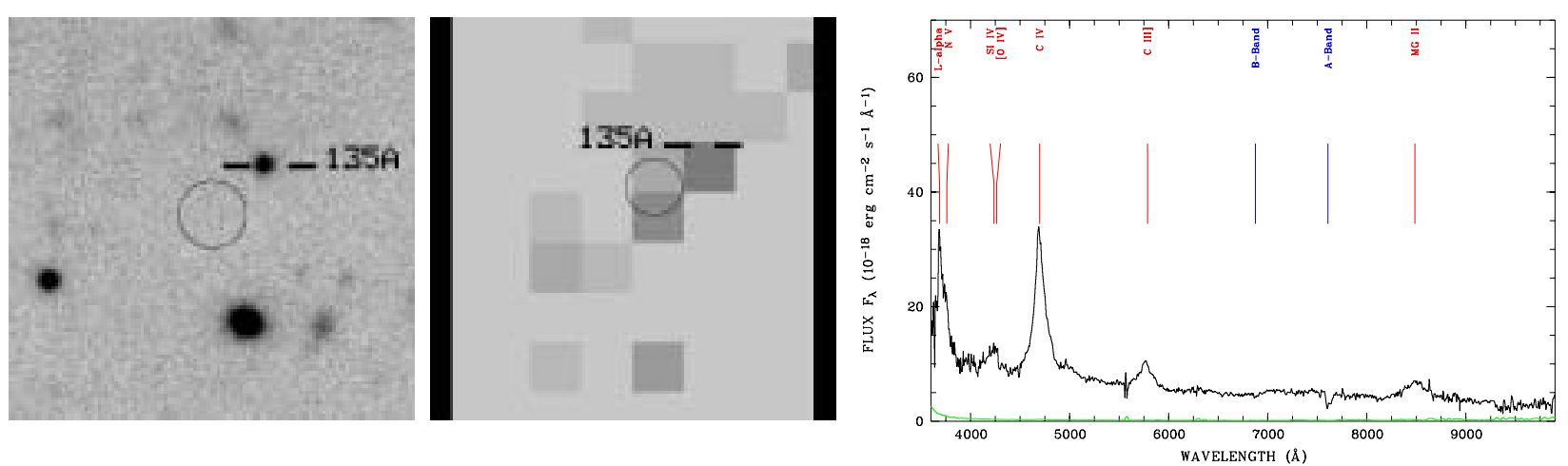

object $135 \mathrm{~A}, \mathrm{z}=2.033$ 
M. Krumpe et al.: The XMM-Newton survey in the Marano field. I., Online Material p 33
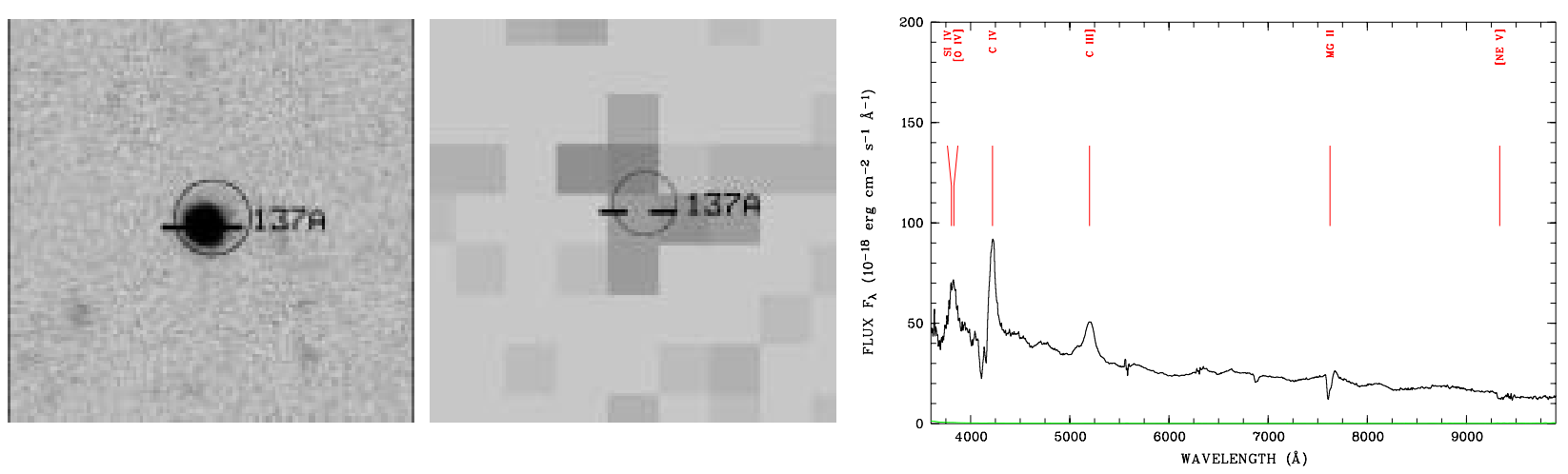

object $137 \mathrm{~A}, \mathrm{z}=1.721$
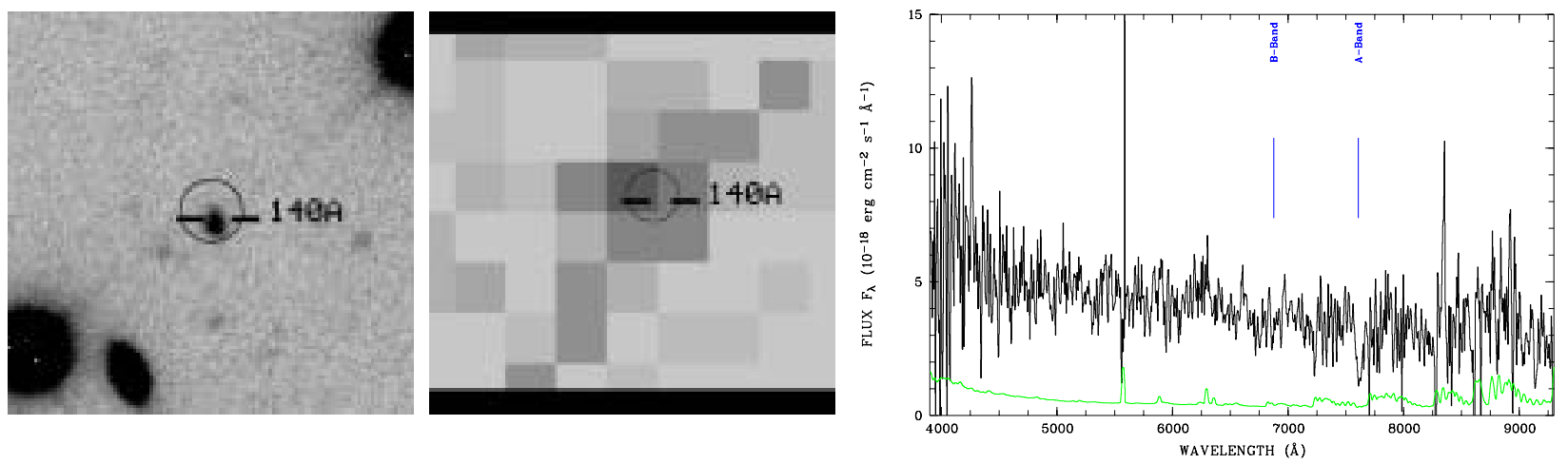

object 140A, no redshift, spectrum too noisy
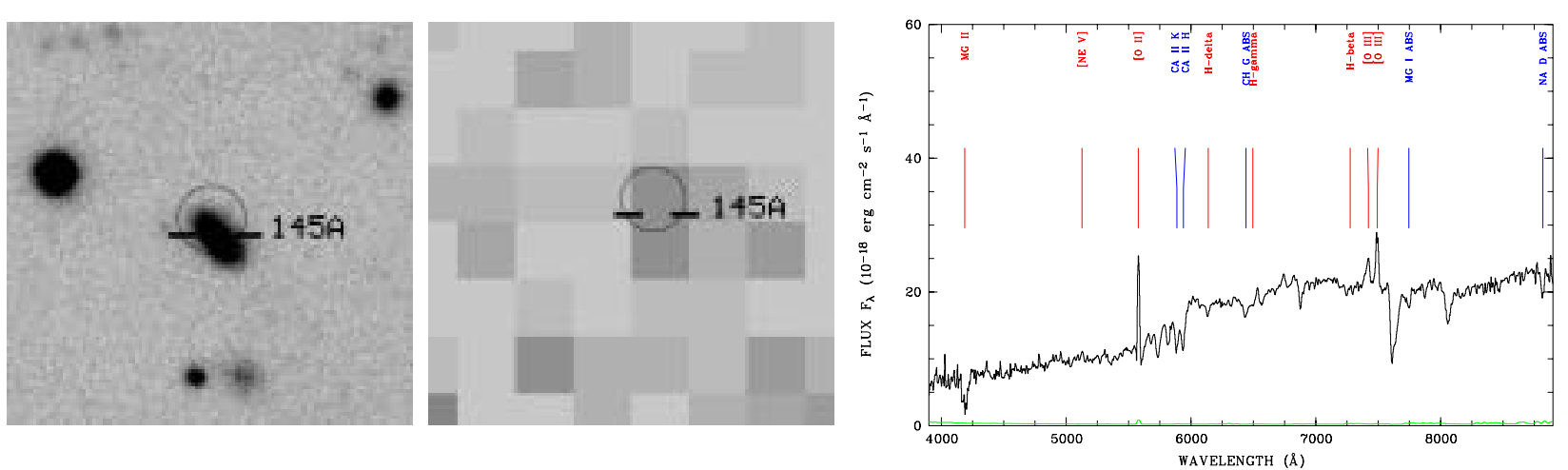

object $145 \mathrm{~A}, \mathrm{z}=0.497$
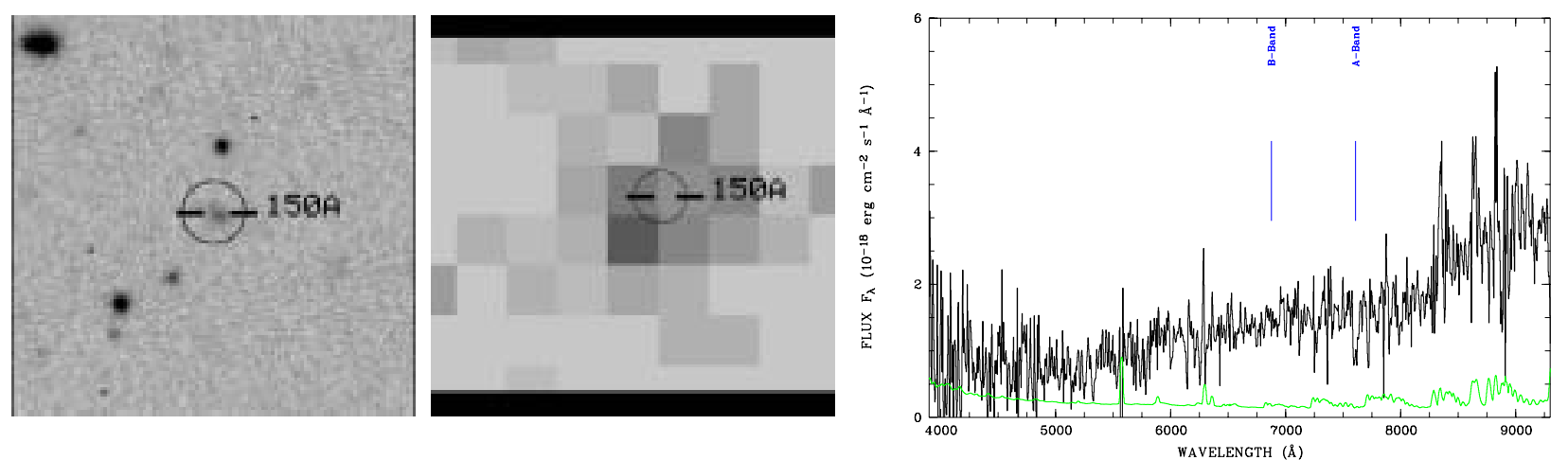

object 150A, no redshift, spectrum too noisy 
M. Krumpe et al.: The XMM-Newton survey in the Marano field. I., Online Material p 34
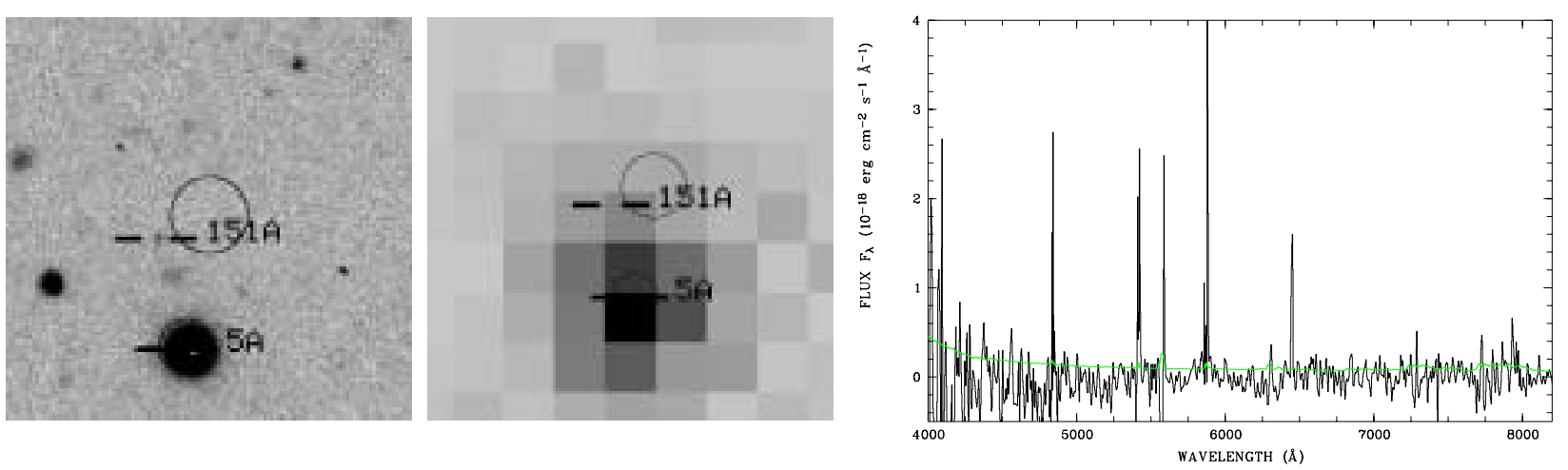

object 151A, no redshift, spectrum too noisy
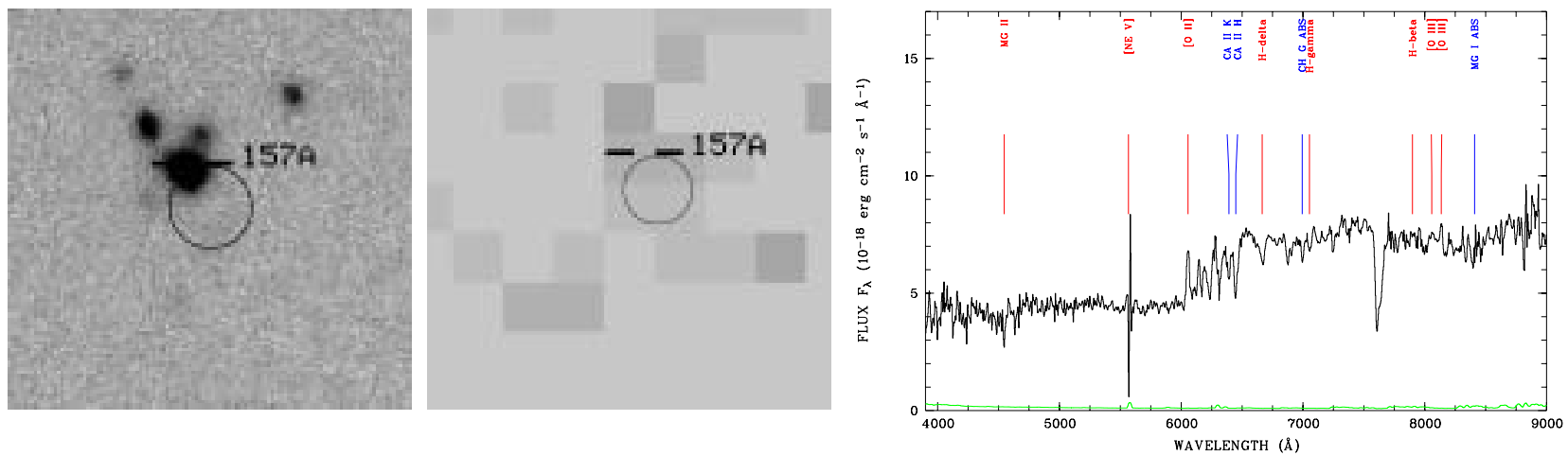

object 157A, $\mathrm{z}=0.625$
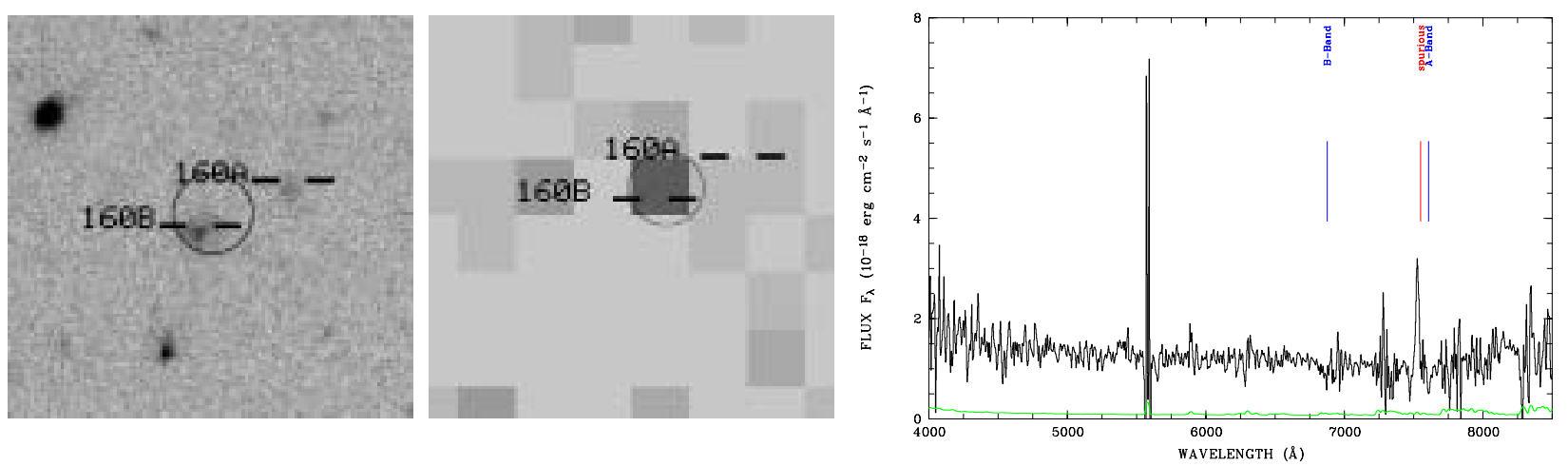

object 160A, no redshift, spectrum too noisy
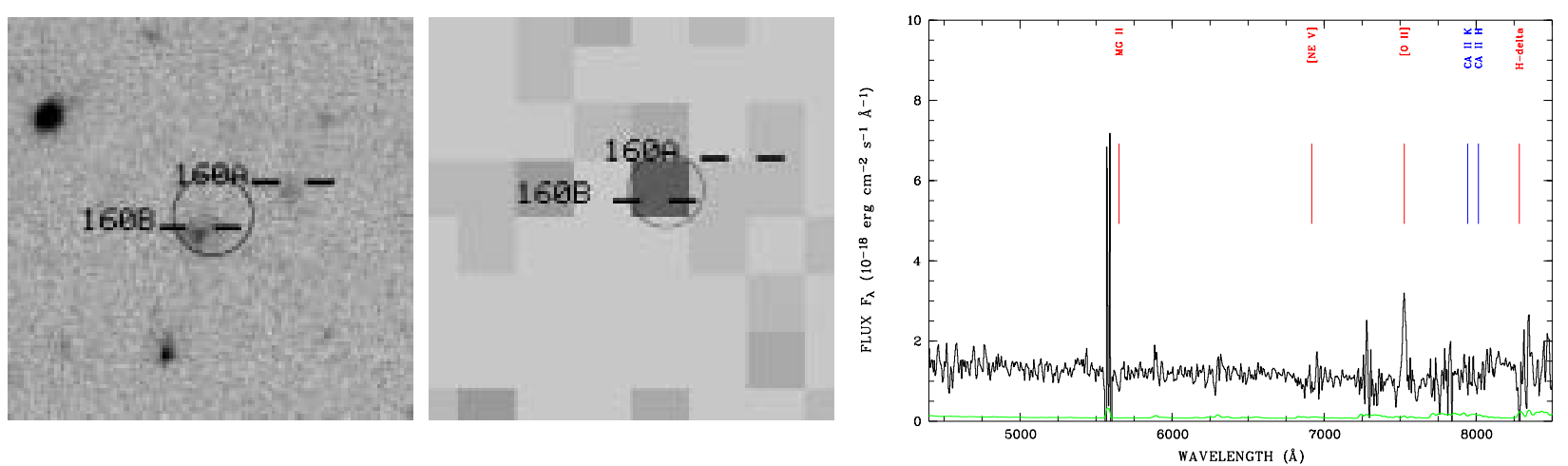

object 160B, z = 1.020:@OII 

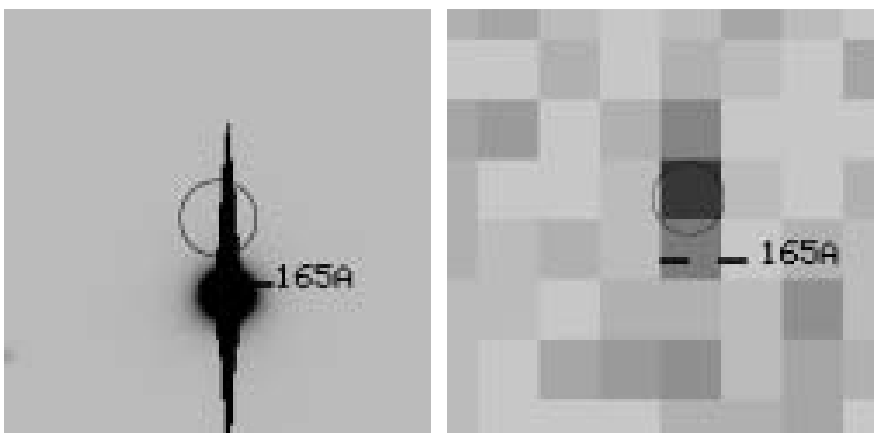

object $165 \mathrm{~A}, \mathrm{z}=0.000$
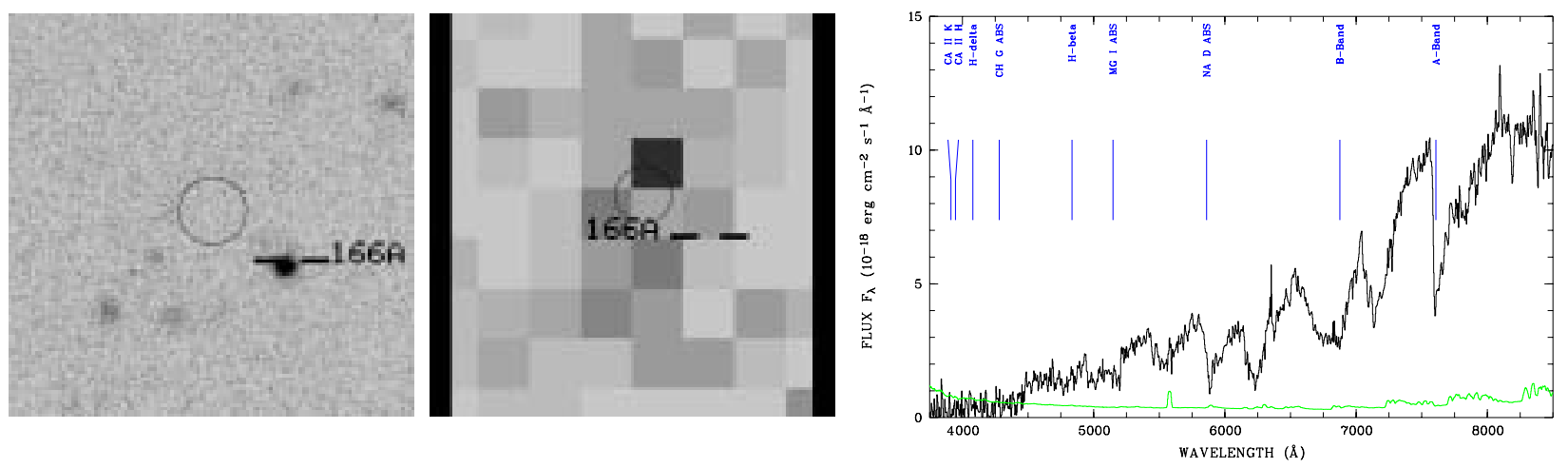

object $166 \mathrm{~A}, \mathrm{z}=0.000$
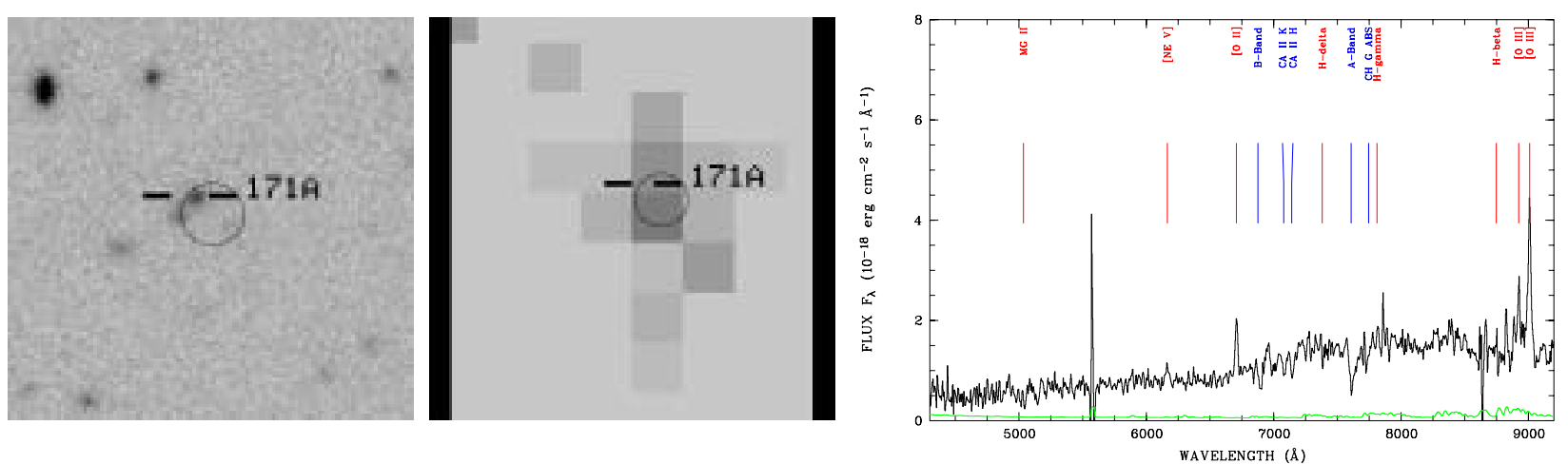

object $171 \mathrm{~A}, \mathrm{z}=0.800$
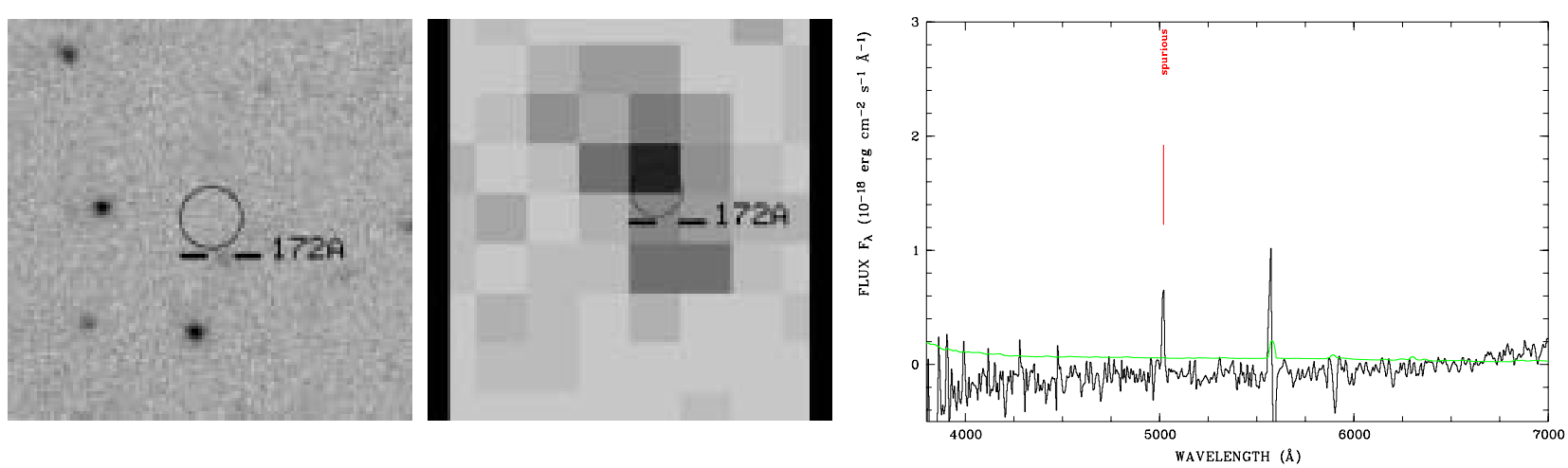

object 172A, no redshift, spectrum too noisy 
M. Krumpe et al.: The XMM-Newton survey in the Marano field. I., Online Material p 36
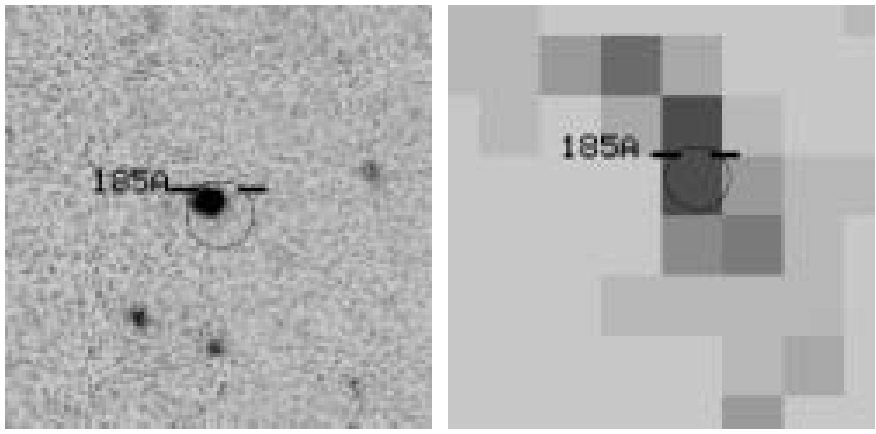

object $185 \mathrm{~A}, \mathrm{z}=2.768$
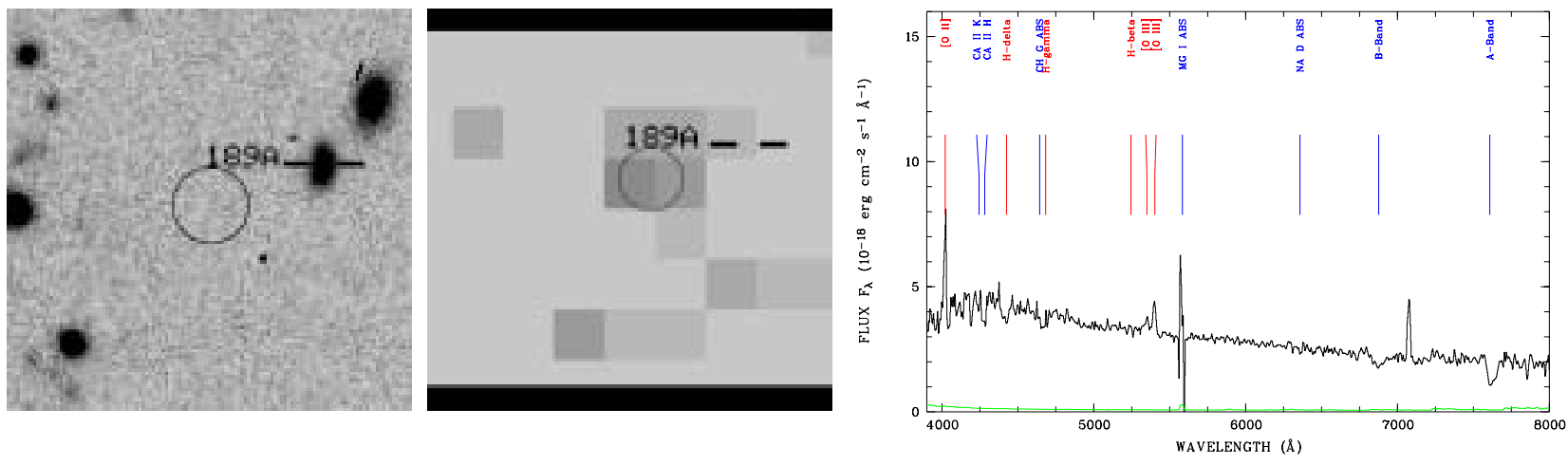

object $189 \mathrm{~A}, \mathrm{z}=0.079$
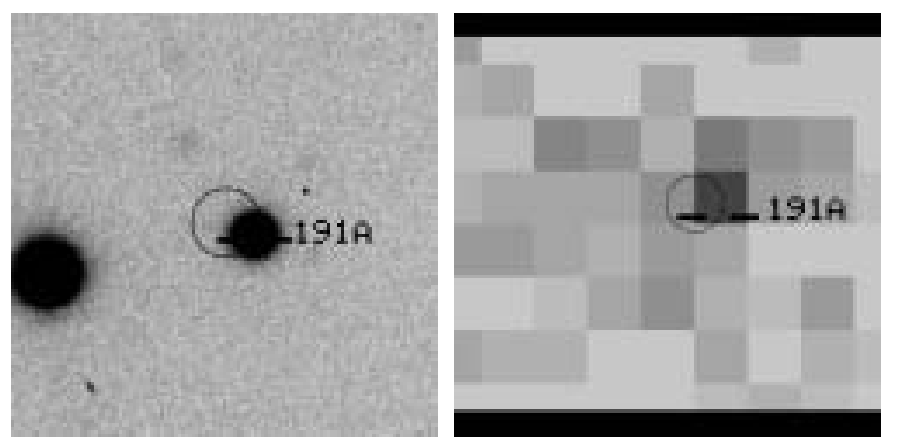

object $191 \mathrm{~A}, \mathrm{z}=1.045$
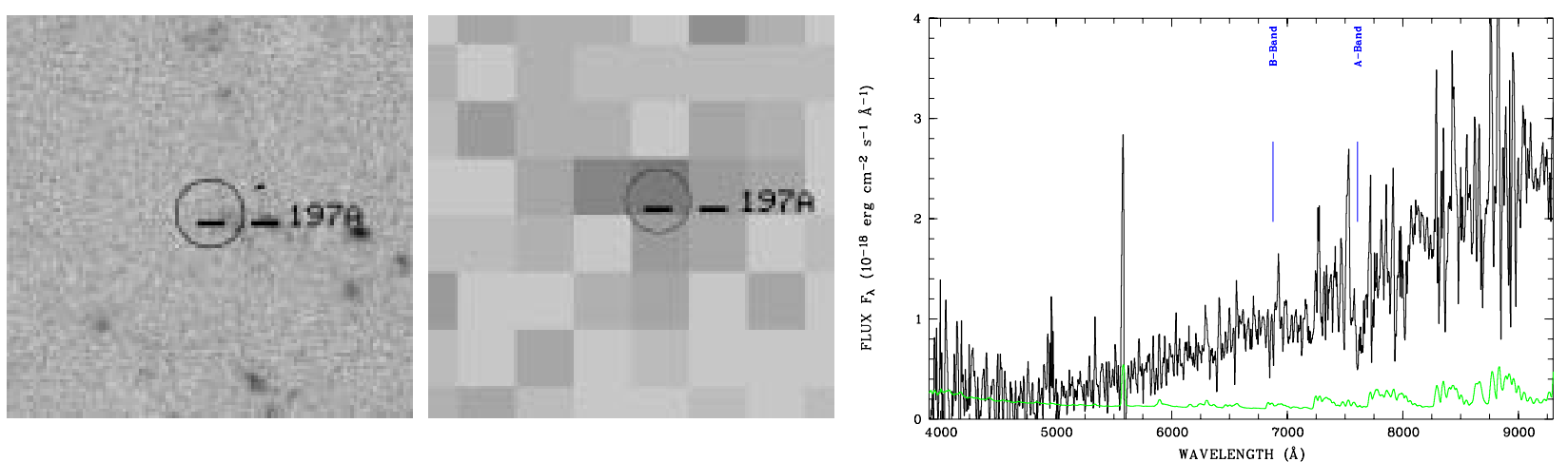

object 197A, no redshift, spectrum too noisy 
M. Krumpe et al.: The XMM-Newton survey in the Marano field. I., Online Material p 37
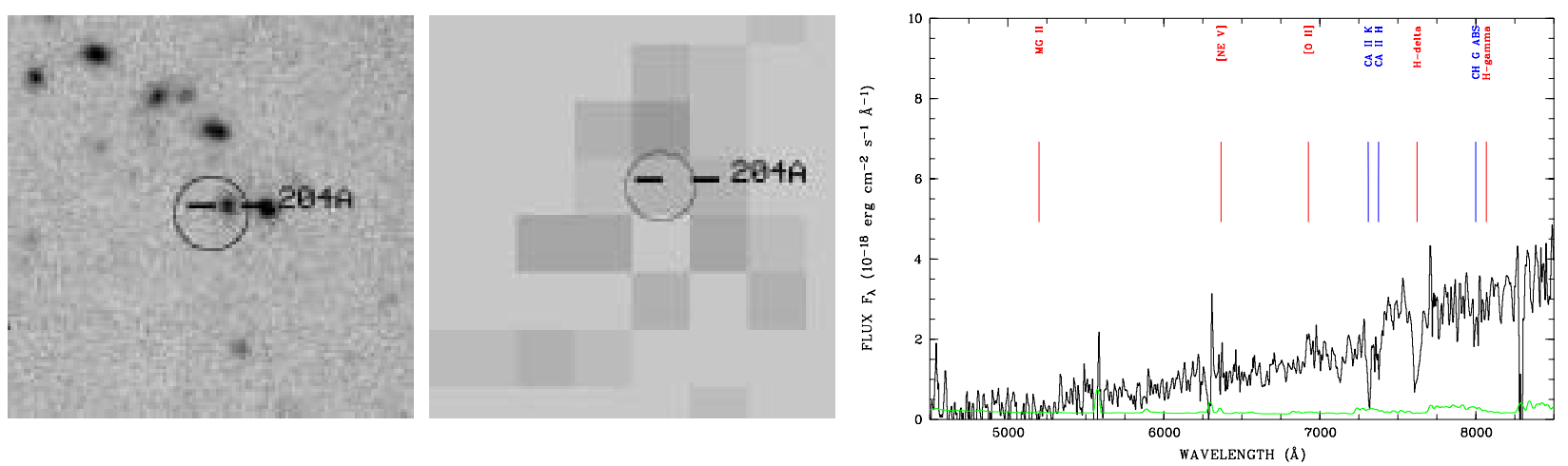

object $204 \mathrm{~A}, \mathrm{z}=0.859$
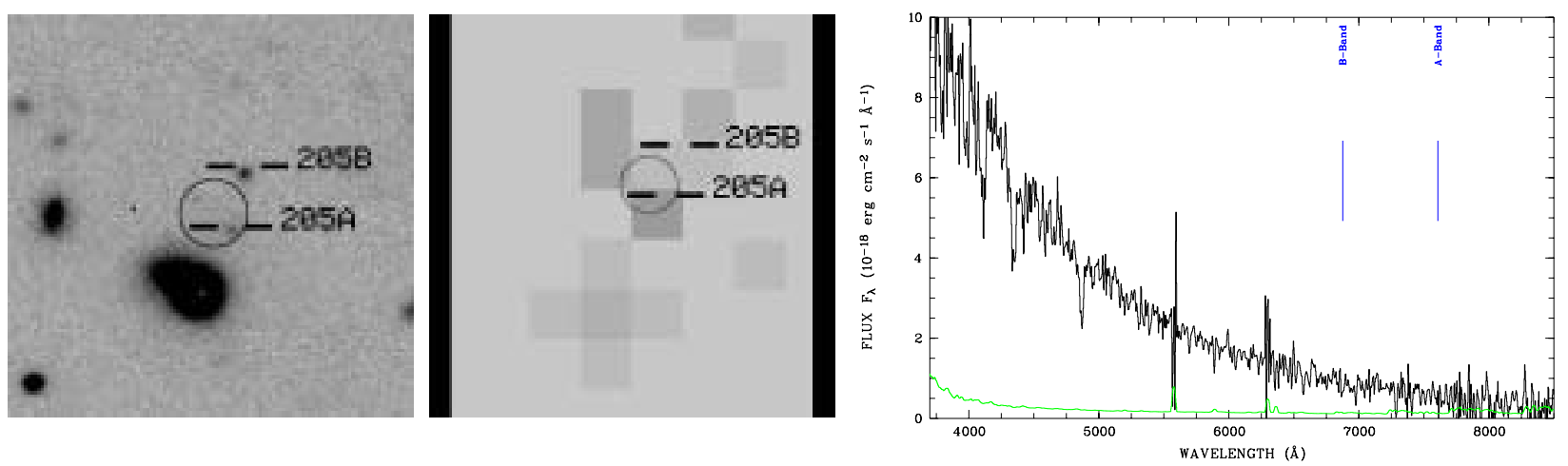

object 205A, z $=0.000$
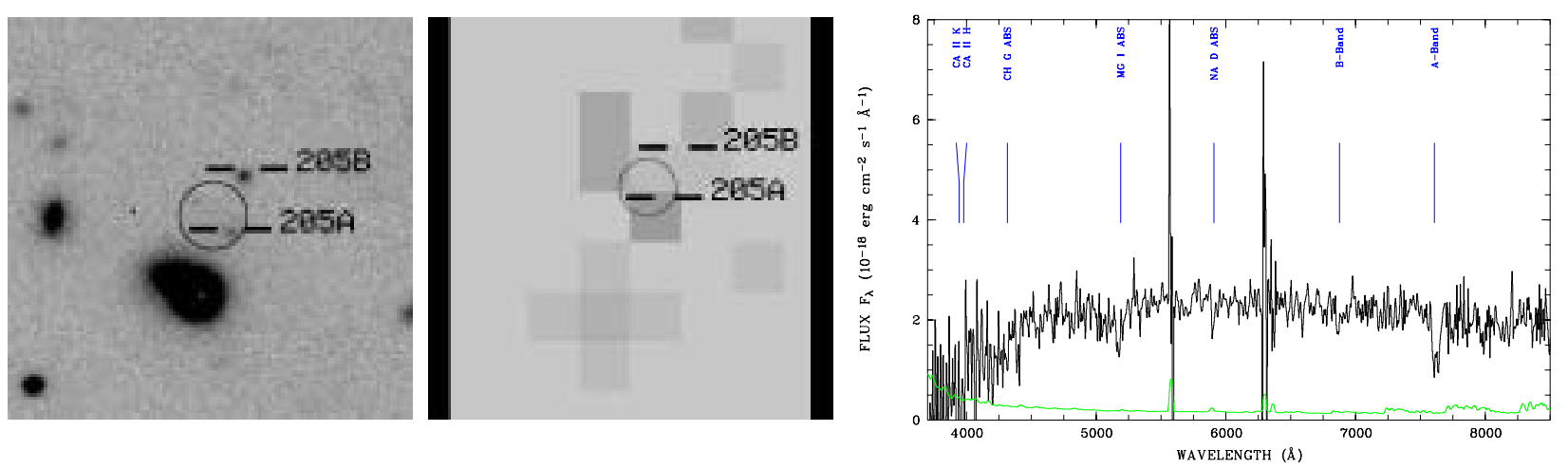

object $205 \mathrm{~B}, \mathrm{z}=0.000$
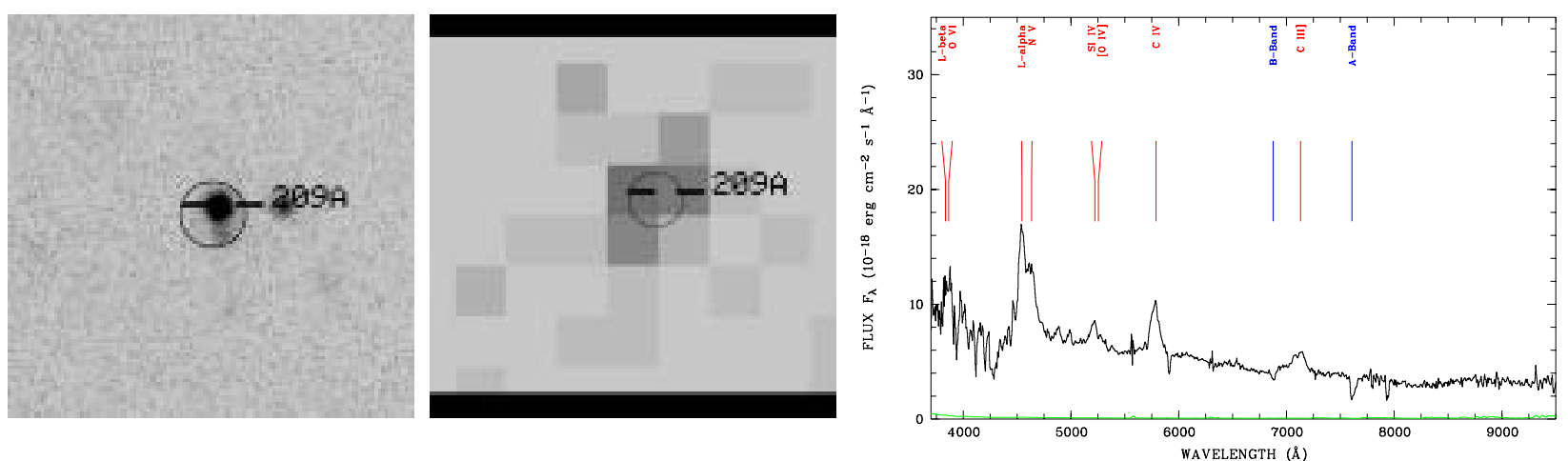

object 209A, $\mathrm{z}=2.735$ 
M. Krumpe et al.: The XMM-Newton survey in the Marano field. I., Online Material p 38
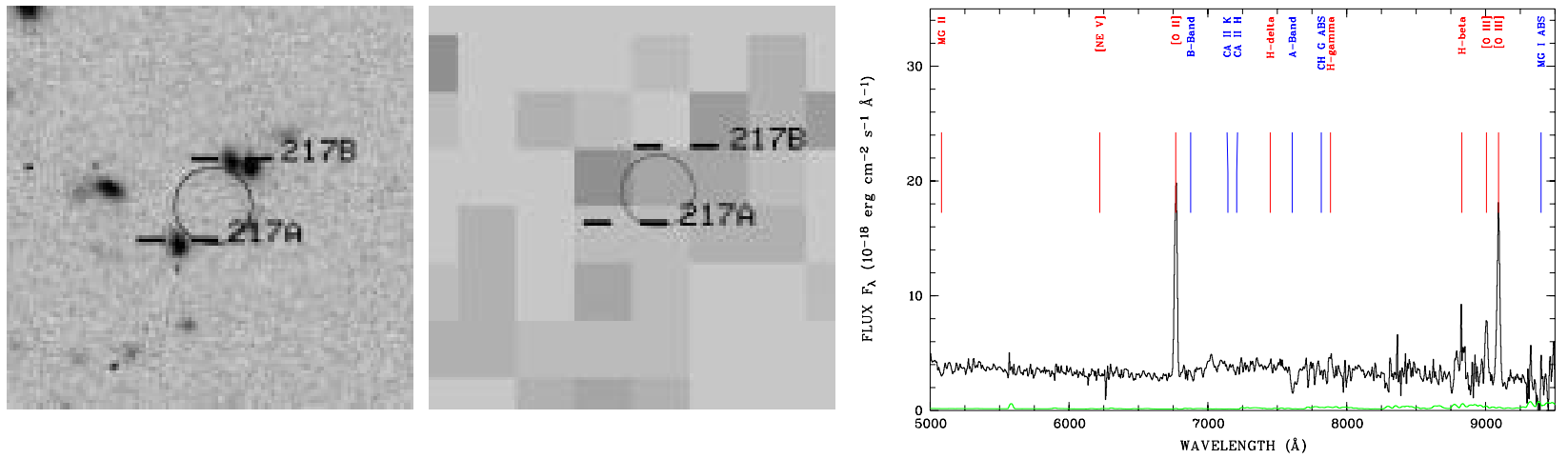

object $217 \mathrm{~A}, \mathrm{z}=0.816$
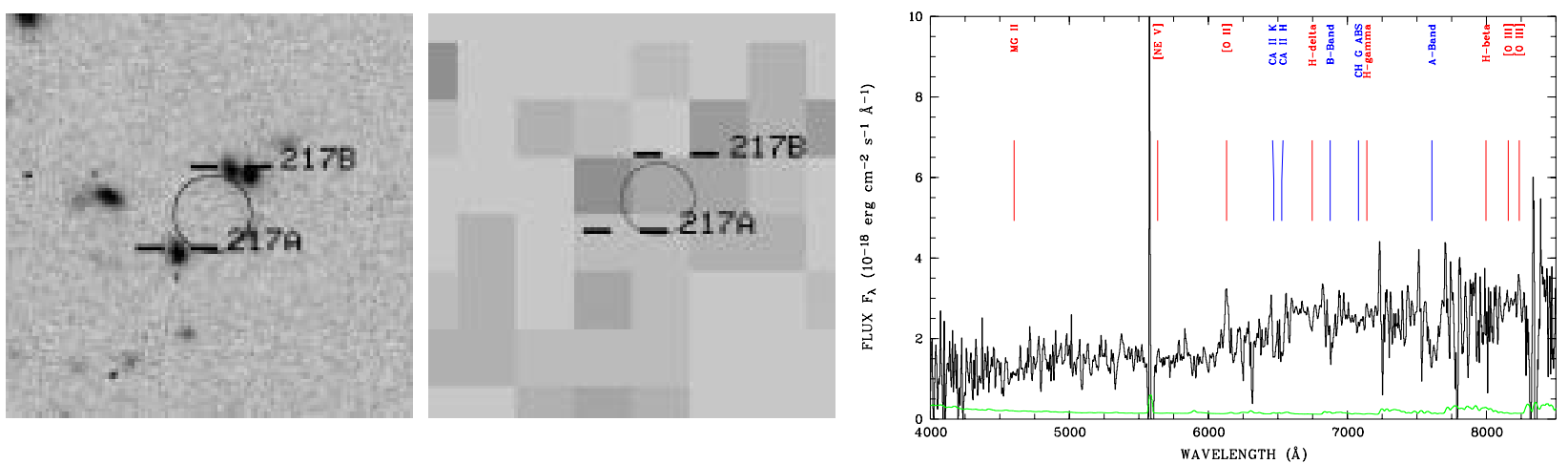

object $217 \mathrm{~B}, \mathrm{z}=0.645$
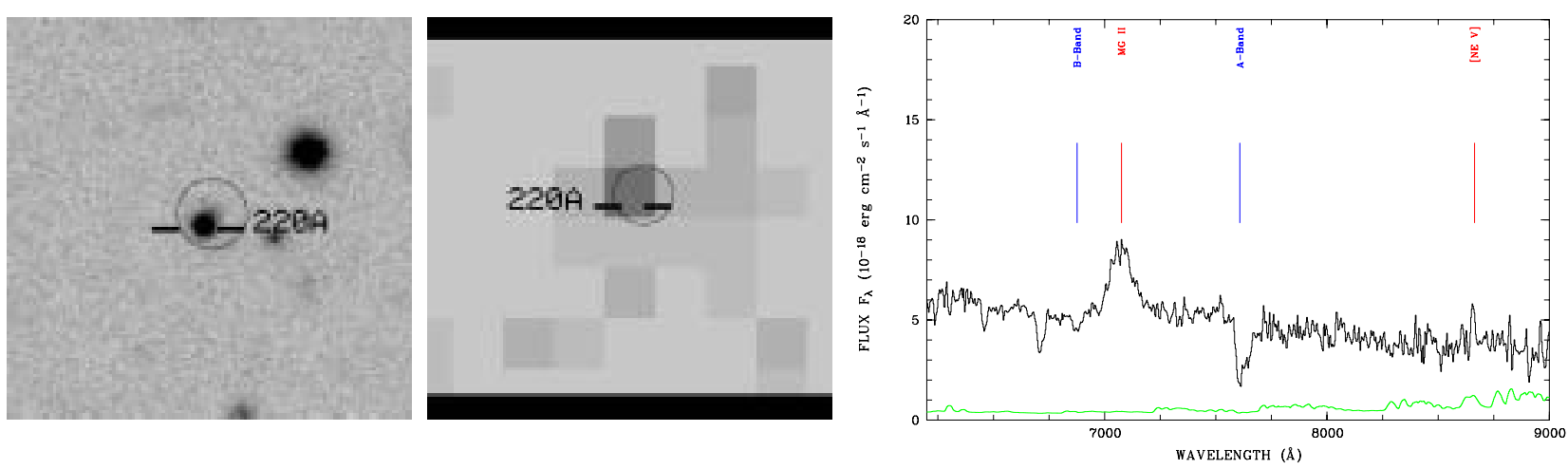

object $220 \mathrm{~A}, \mathrm{z}=1.529$
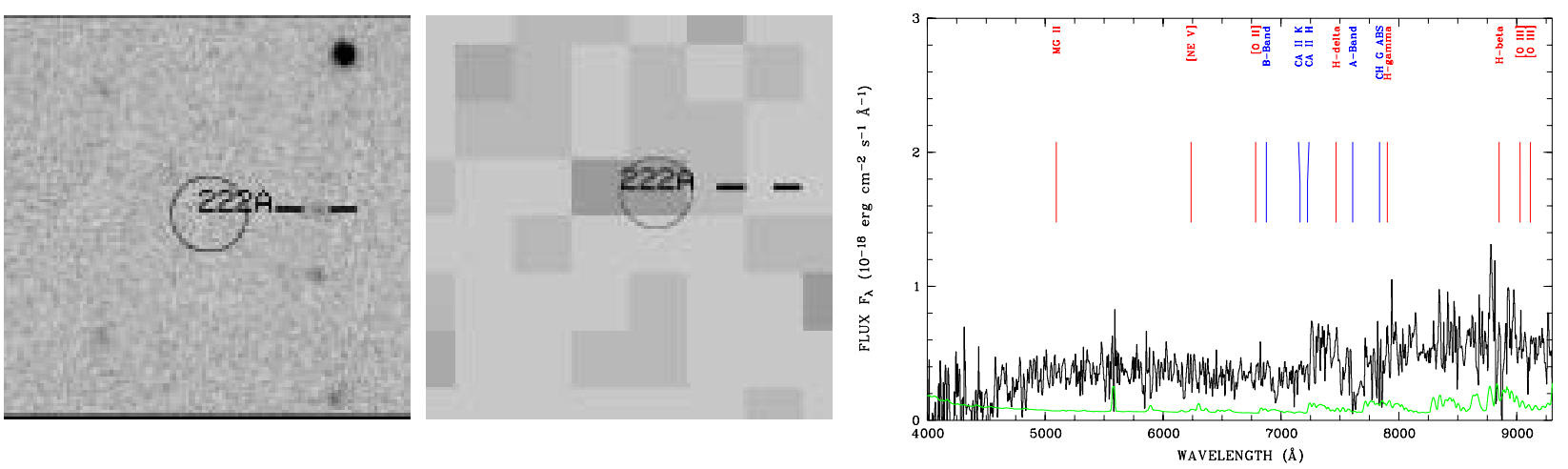

object 222A, z=0.82:@Ca-Break 
M. Krumpe et al.: The XMM-Newton survey in the Marano field. I., Online Material p 39
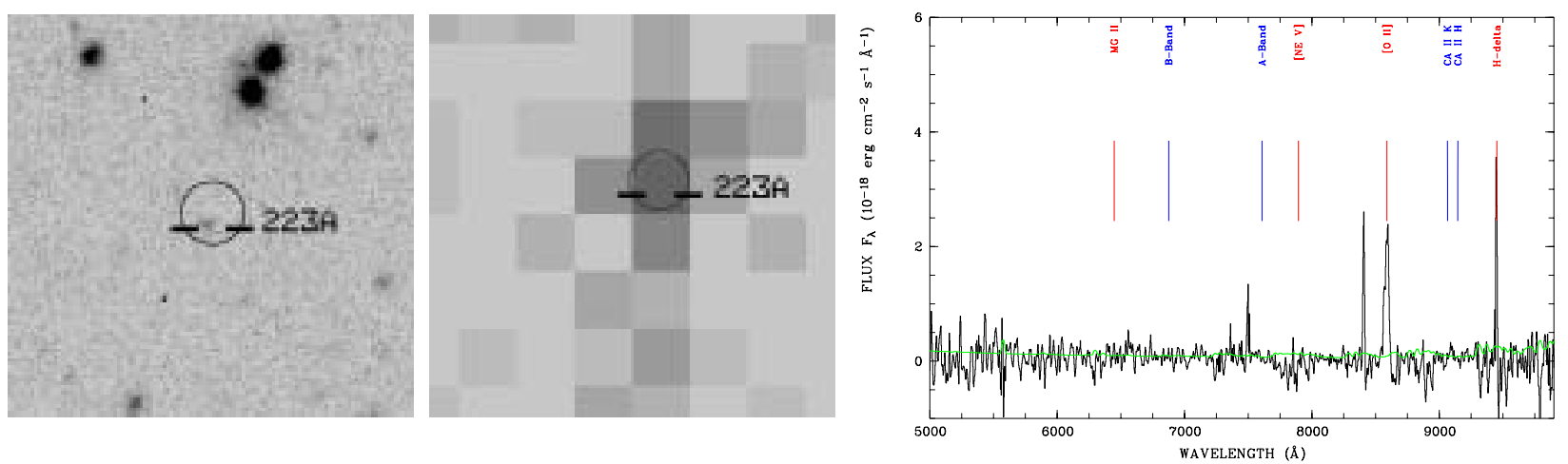

object 223A, z=1.304:@OII
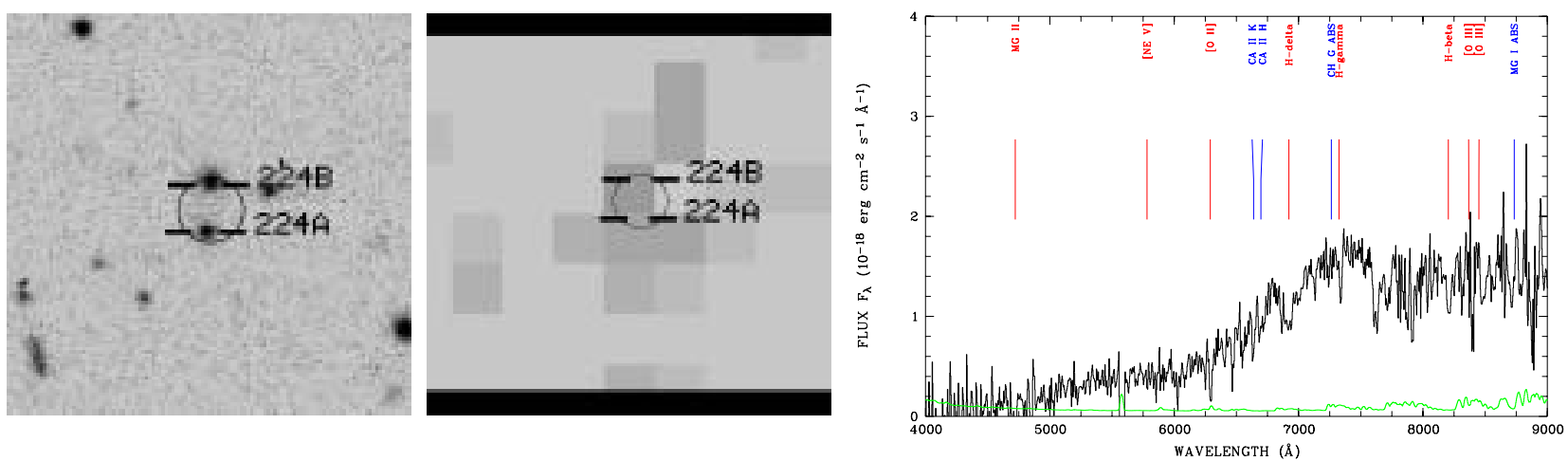

object 224A, z =0.688:@Ca-Break
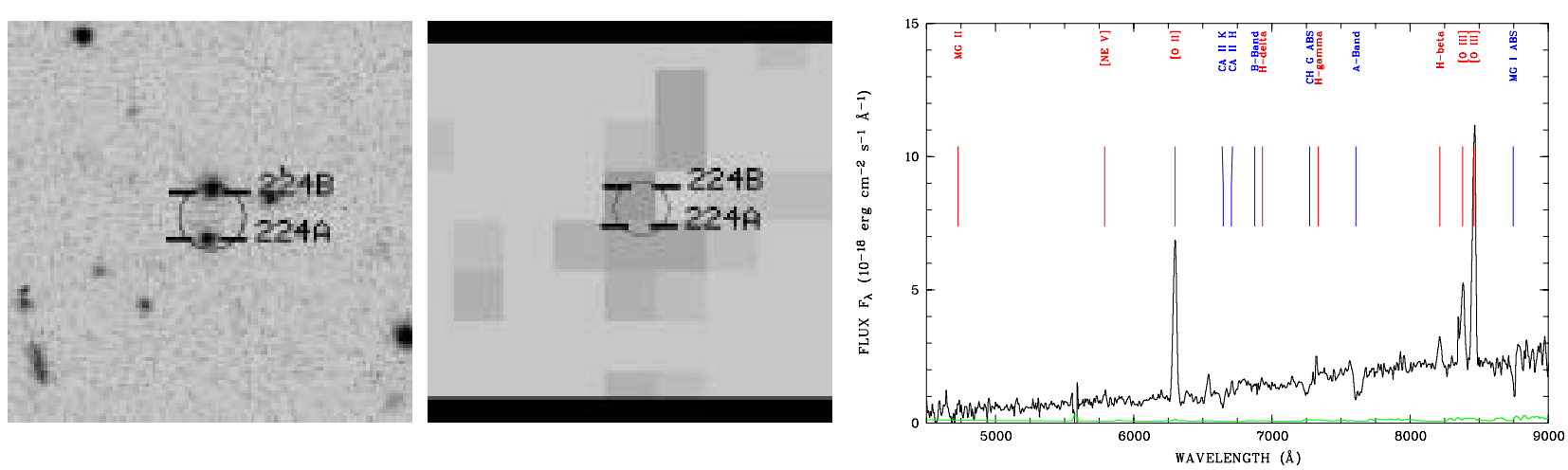

object $224 \mathrm{~B}, \mathrm{z}=0.690$
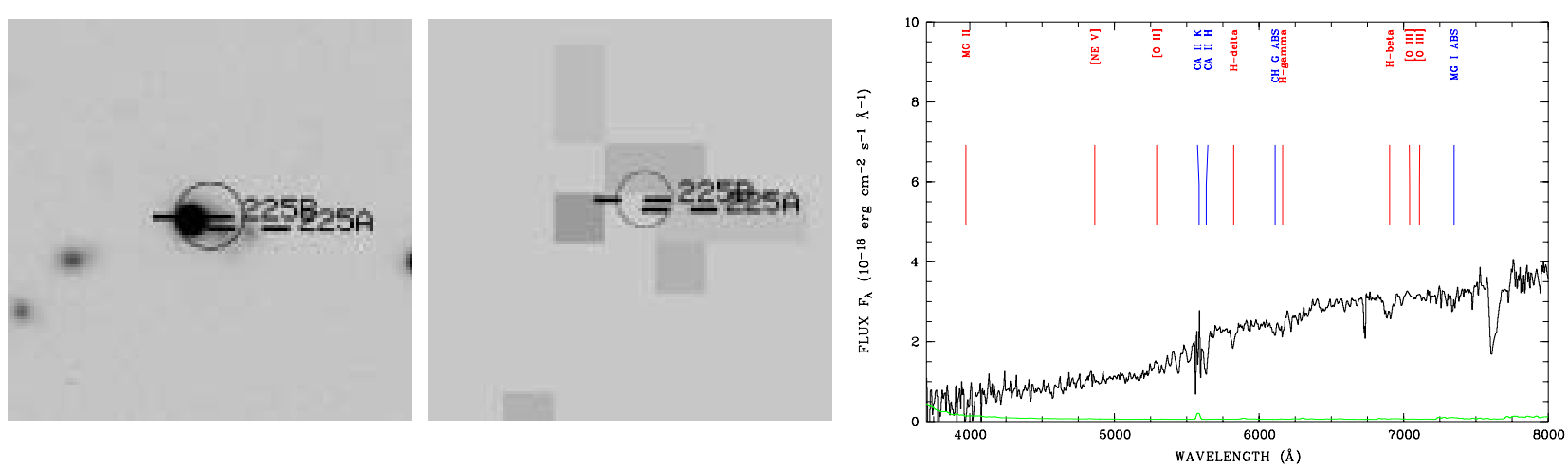

object $225 \mathrm{~A}, \mathrm{z}=0.420$ 
M. Krumpe et al.: The XMM-Newton survey in the Marano field. I., Online Material p 40
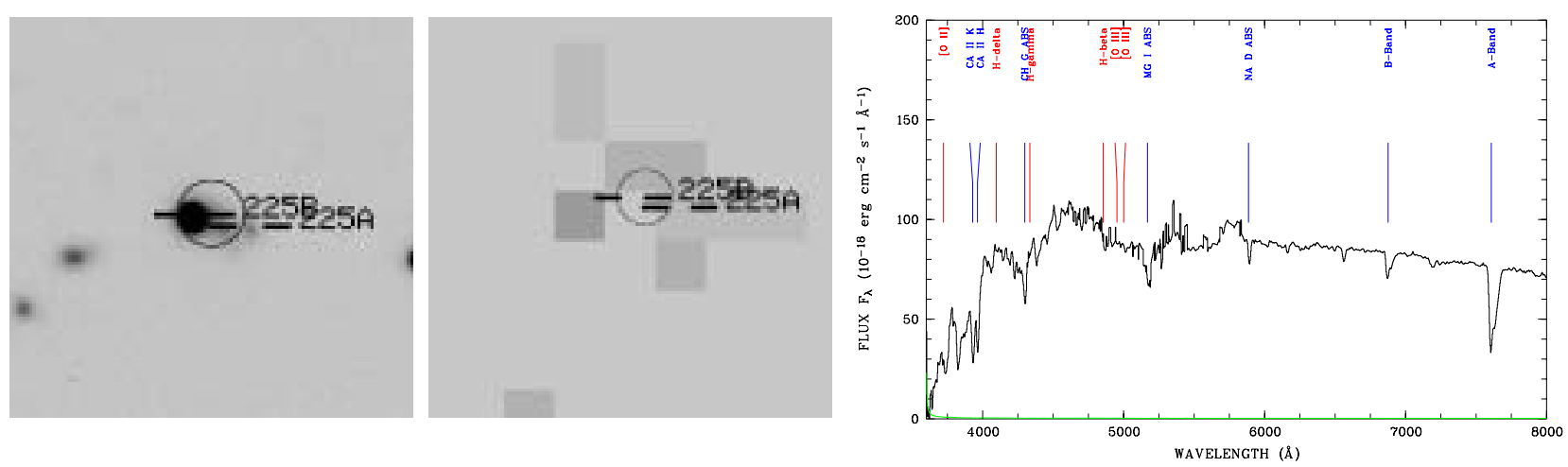

object $225 \mathrm{~B}, \mathrm{z}=0.000$
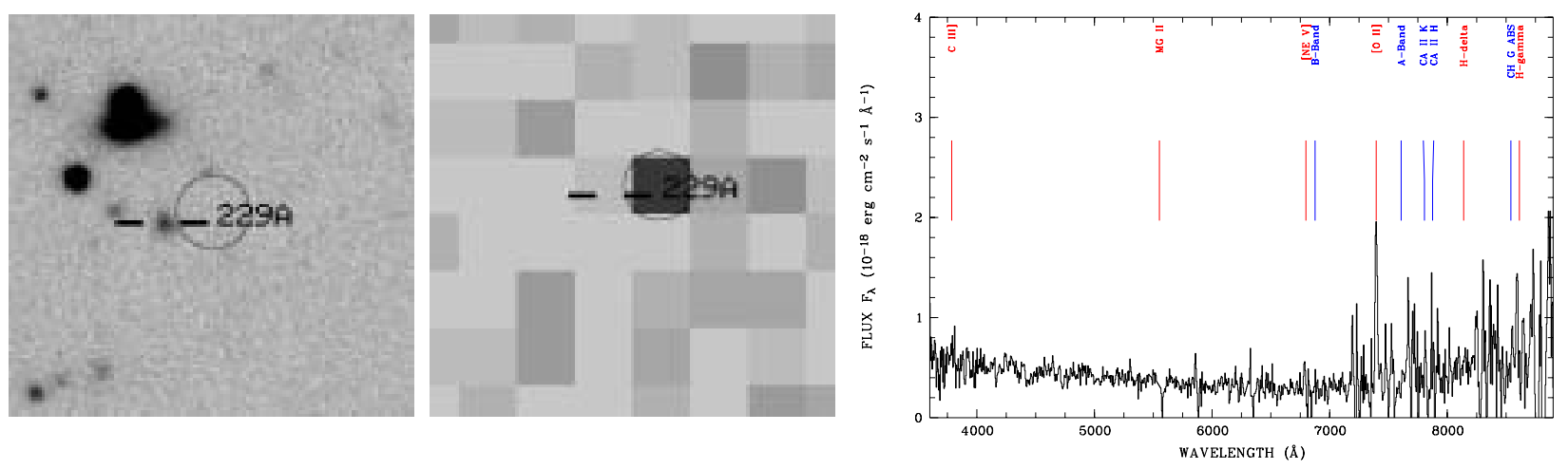

object 229A, z =0.98:@OII
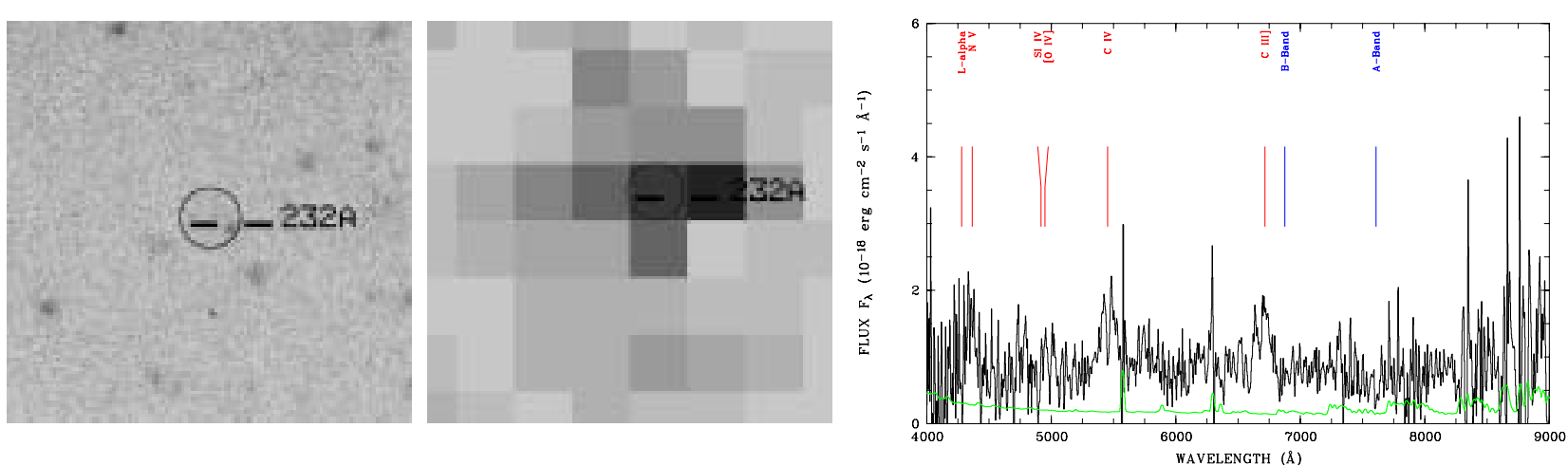

object 232A, $\mathrm{z}=2.520$
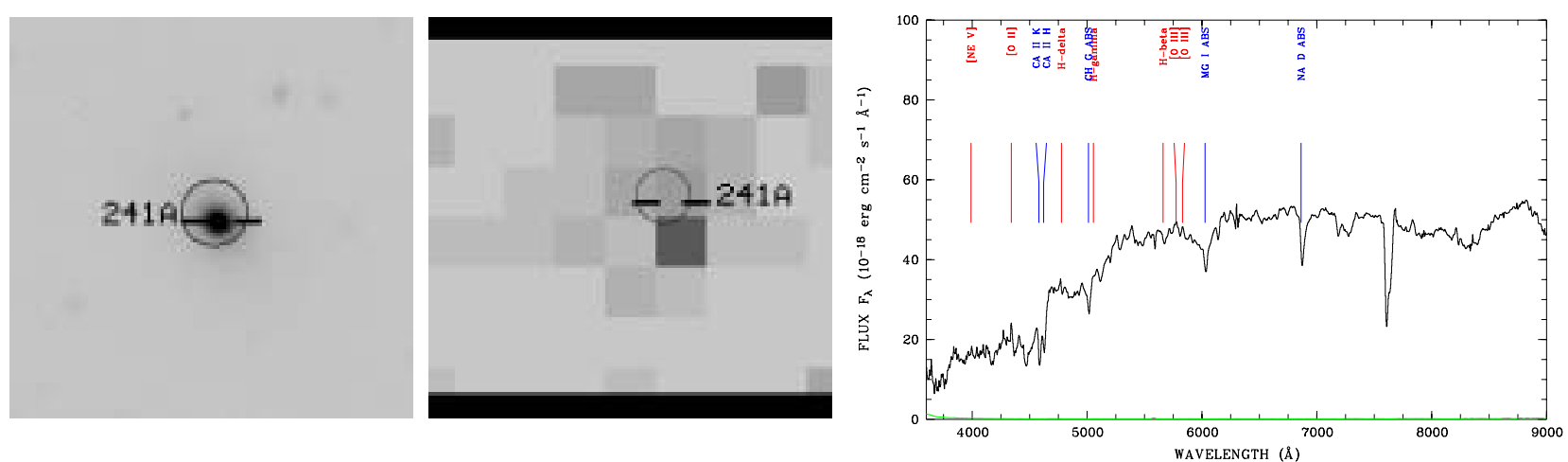

object $241 \mathrm{~A}, \mathrm{z}=0.165$ 
M. Krumpe et al.: The XMM-Newton survey in the Marano field. I., Online Material p 41
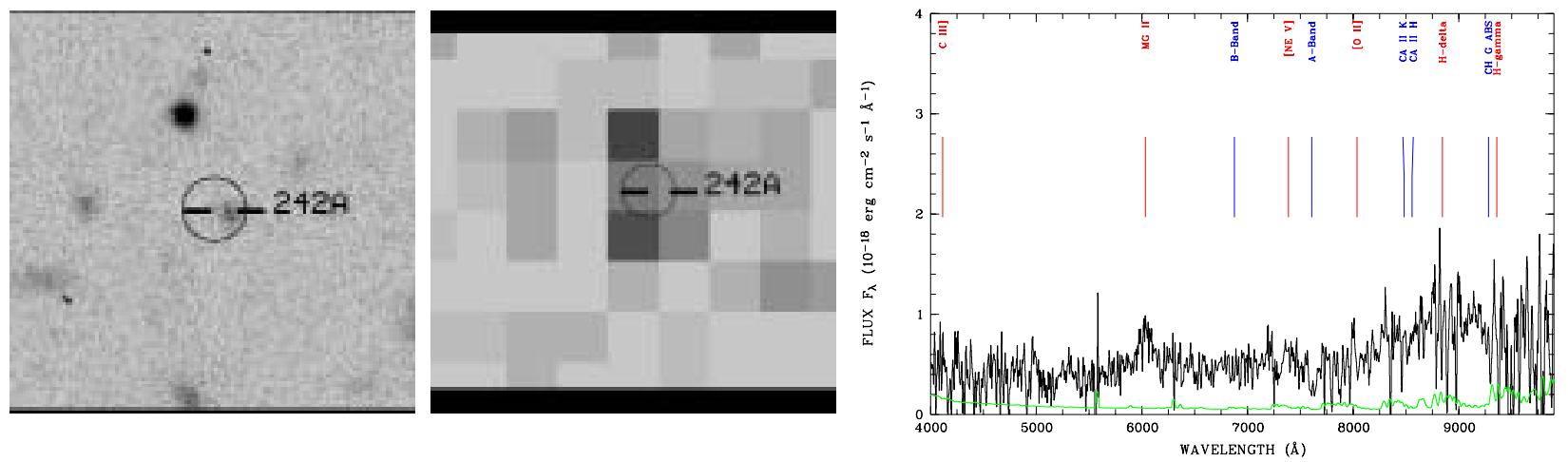

object $242 \mathrm{~A}, \mathrm{z}=1.147$
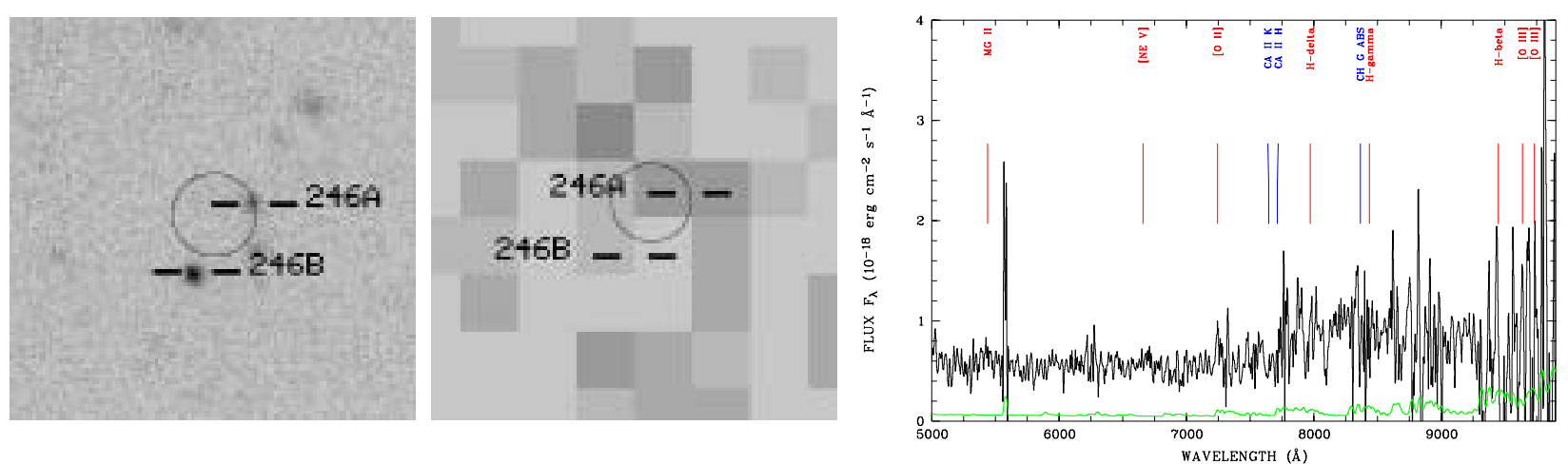

object $246 \mathrm{~A}, \mathrm{z}=0.944$
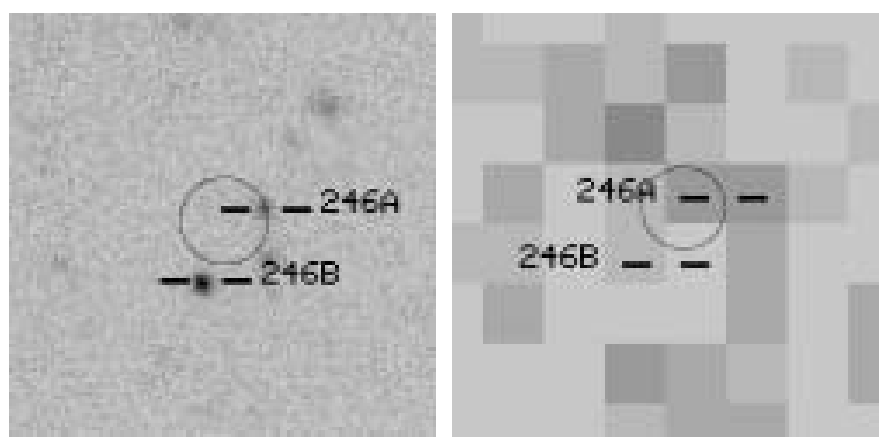

object $246 \mathrm{~B}, \mathrm{z}=0.688$
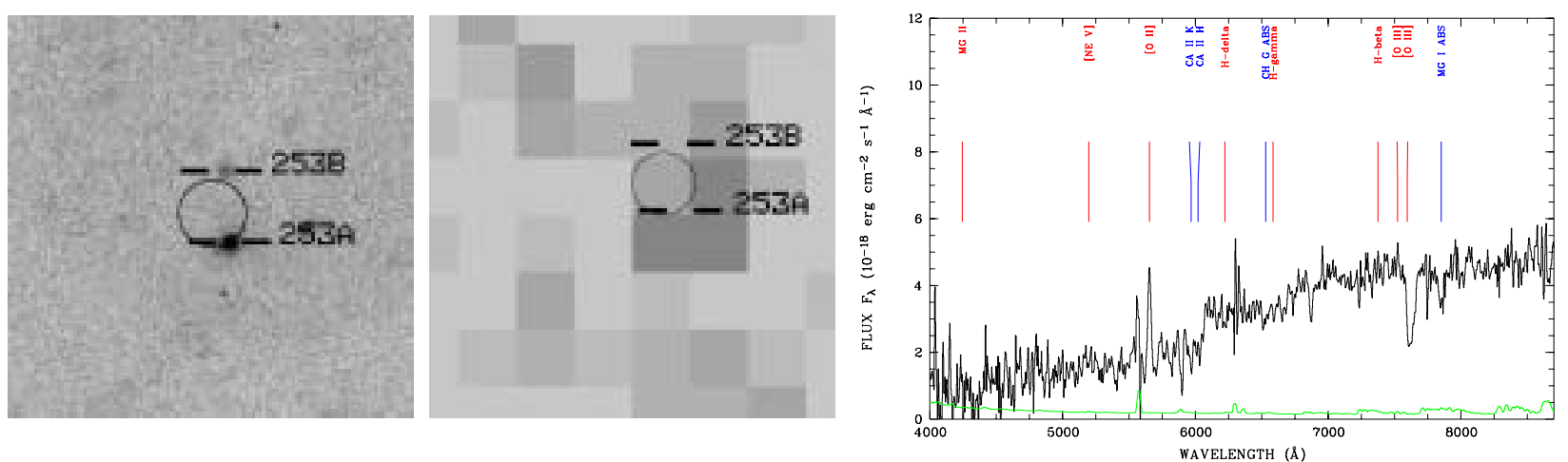

object 253A, $\mathrm{z}=0.517$ 
M. Krumpe et al.: The XMM-Newton survey in the Marano field. I., Online Material p 42
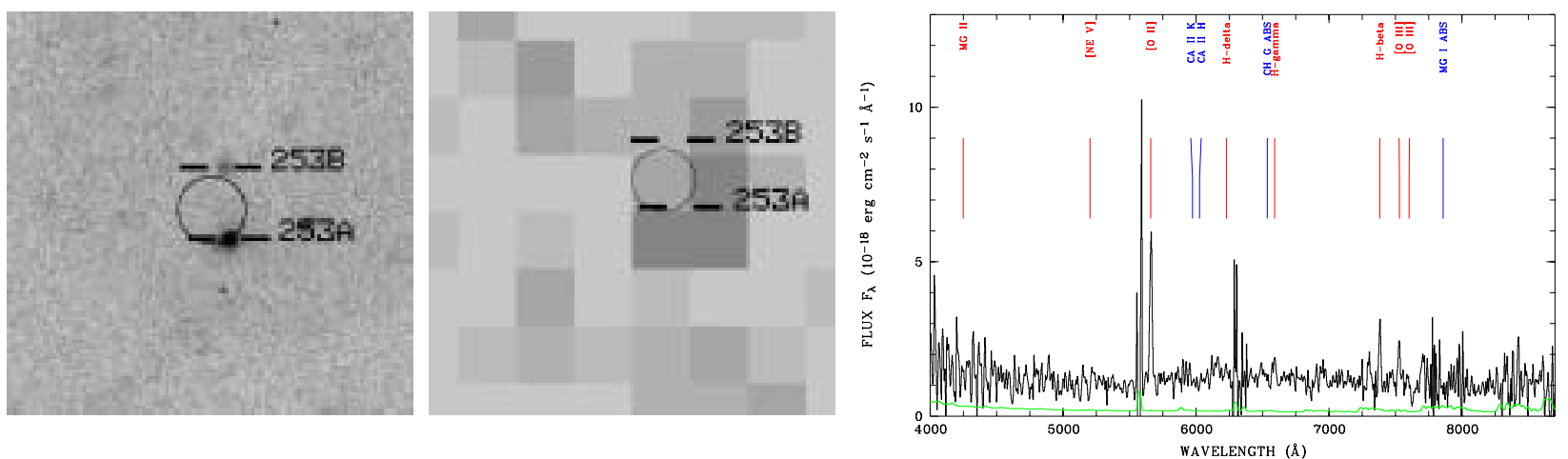

object $253 \mathrm{~B}, \mathrm{z}=0.518$
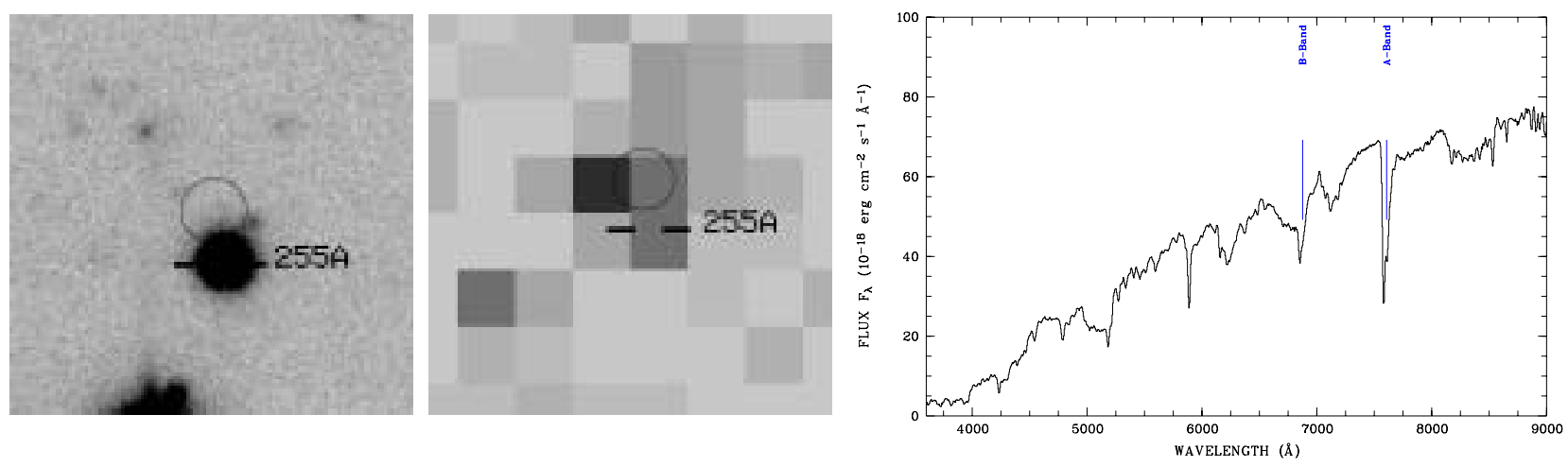

object $255 \mathrm{~A}, \mathrm{z}=0.00$
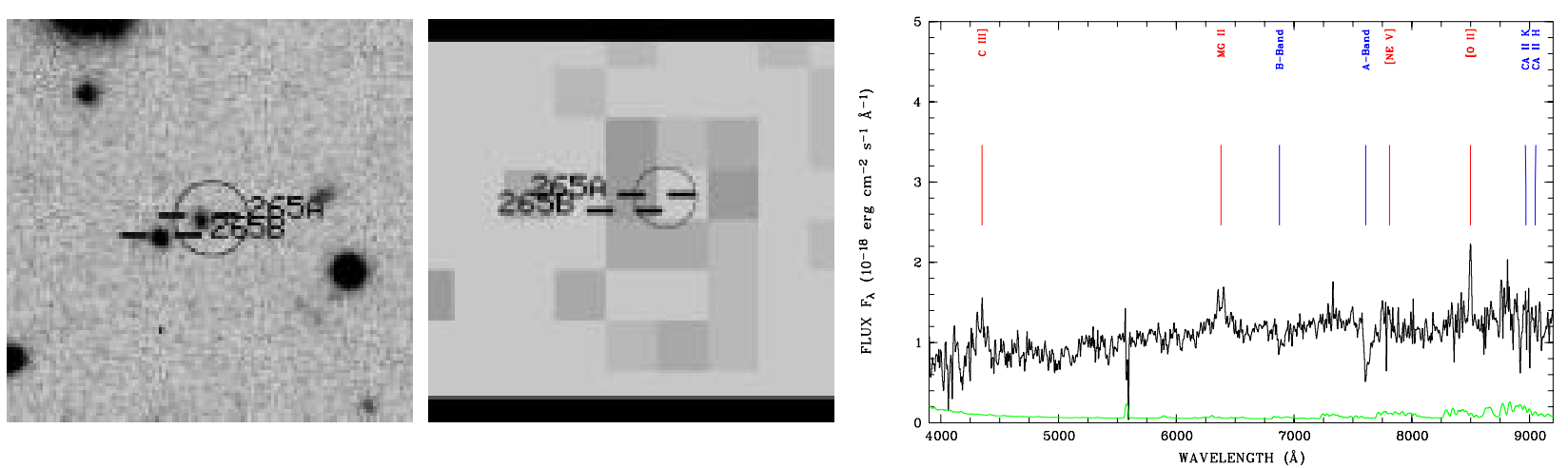

object $265 \mathrm{~A}, \mathrm{z}=1.280$
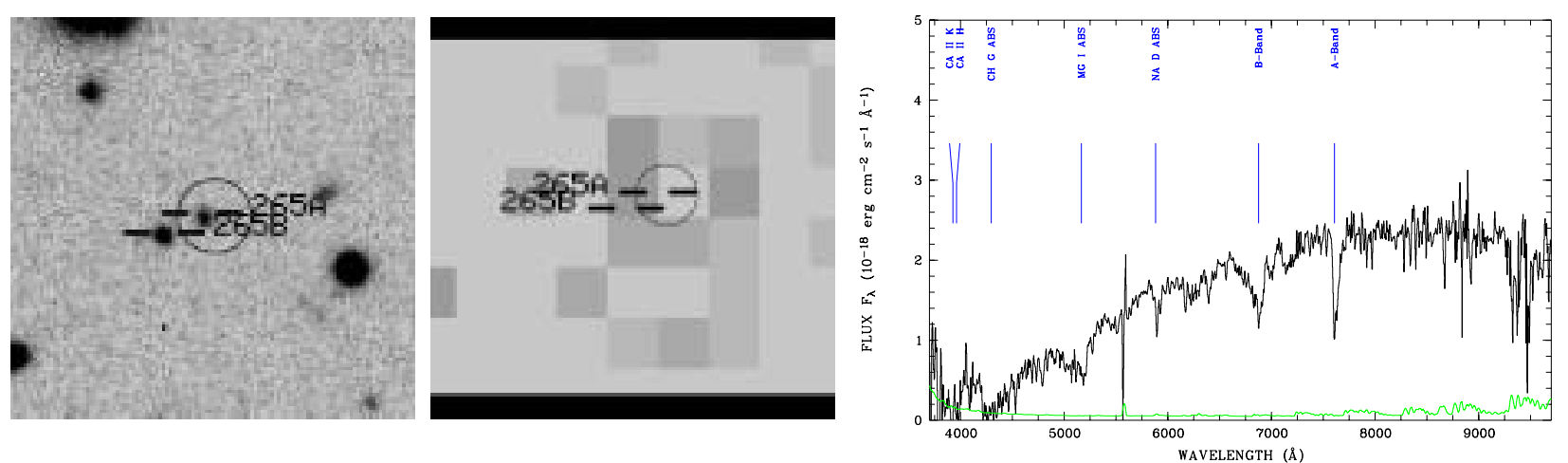

object $265 \mathrm{~B}, \mathrm{z}=0.000$ 
M. Krumpe et al.: The XMM-Newton survey in the Marano field. I., Online Material p 43
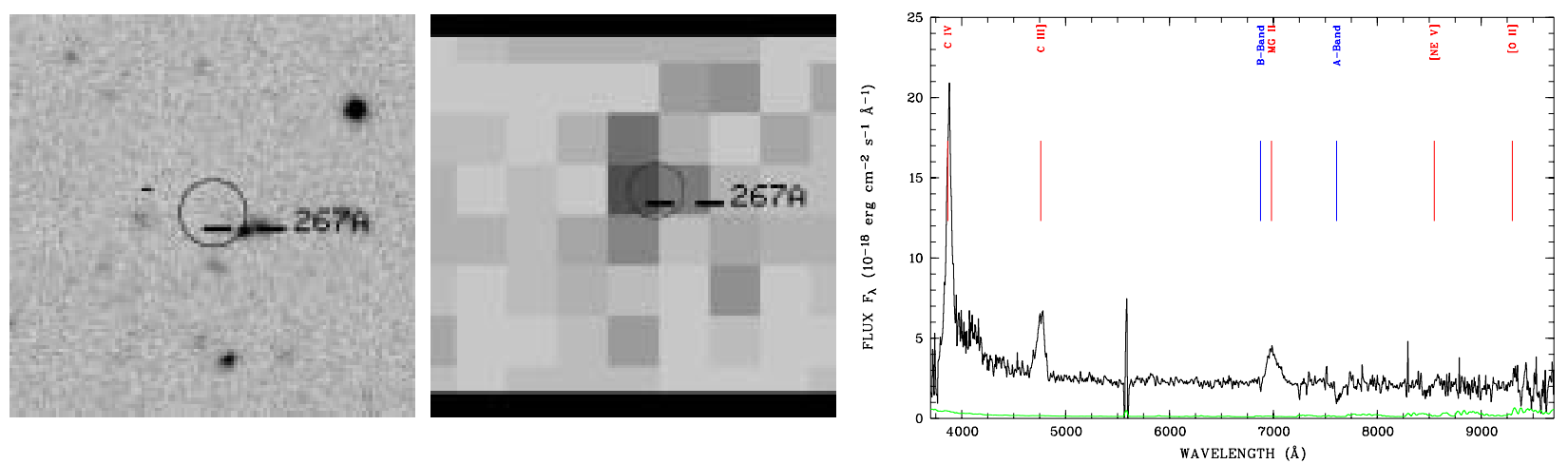

object $267 \mathrm{~A}, \mathrm{z}=1.495$
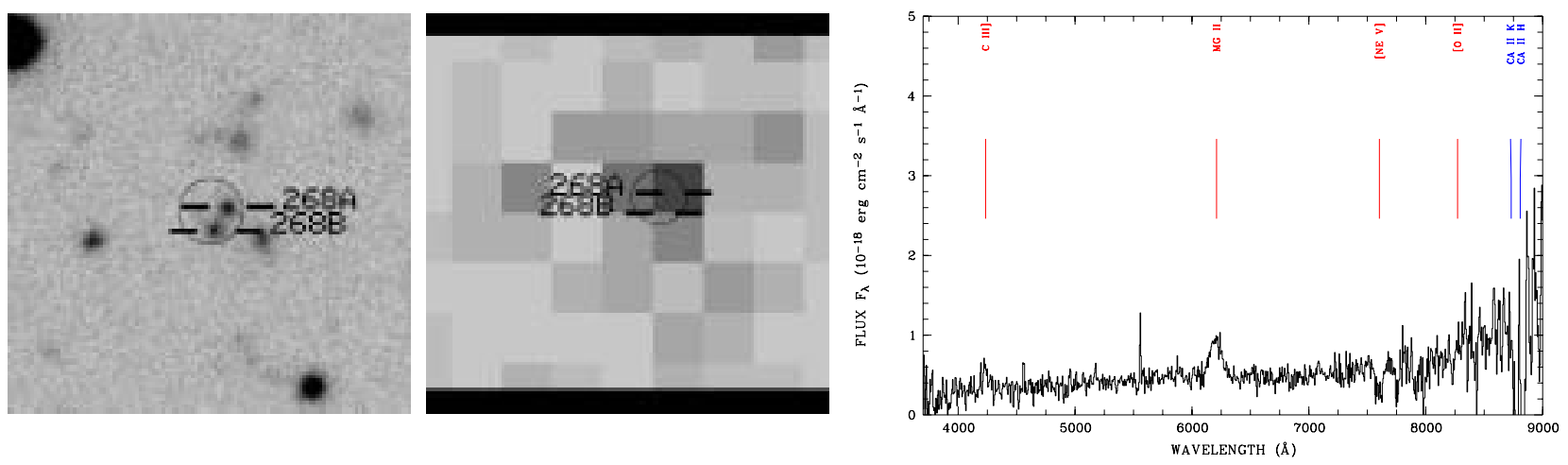

object $268 \mathrm{~A}, \mathrm{z}=1.22$
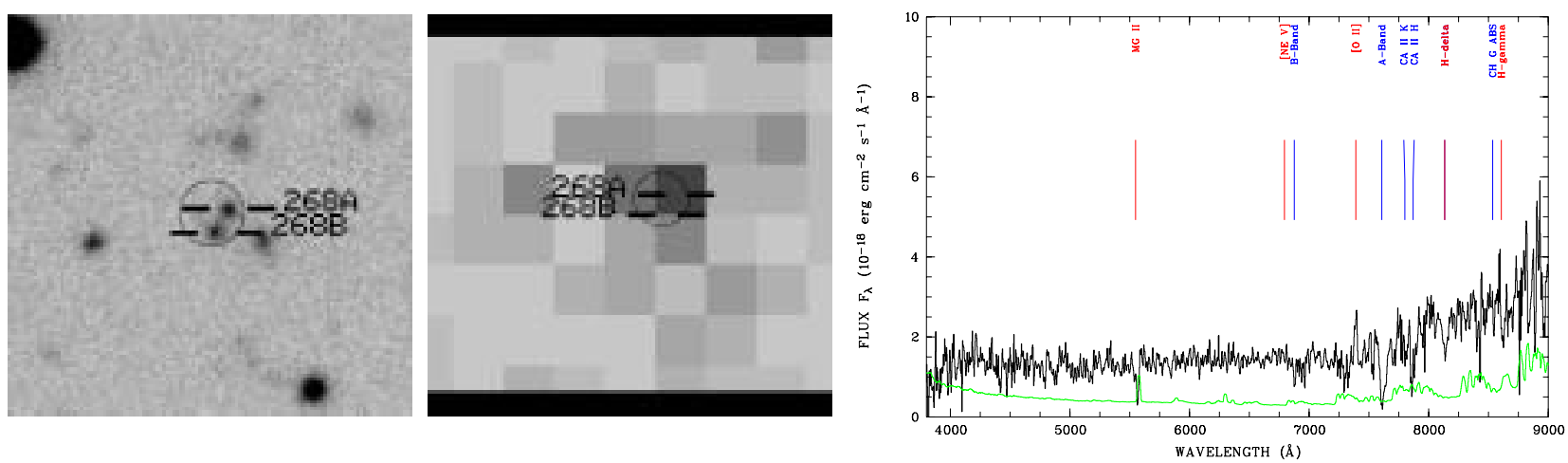

object $268 \mathrm{~B}, \mathrm{z}=0.983$
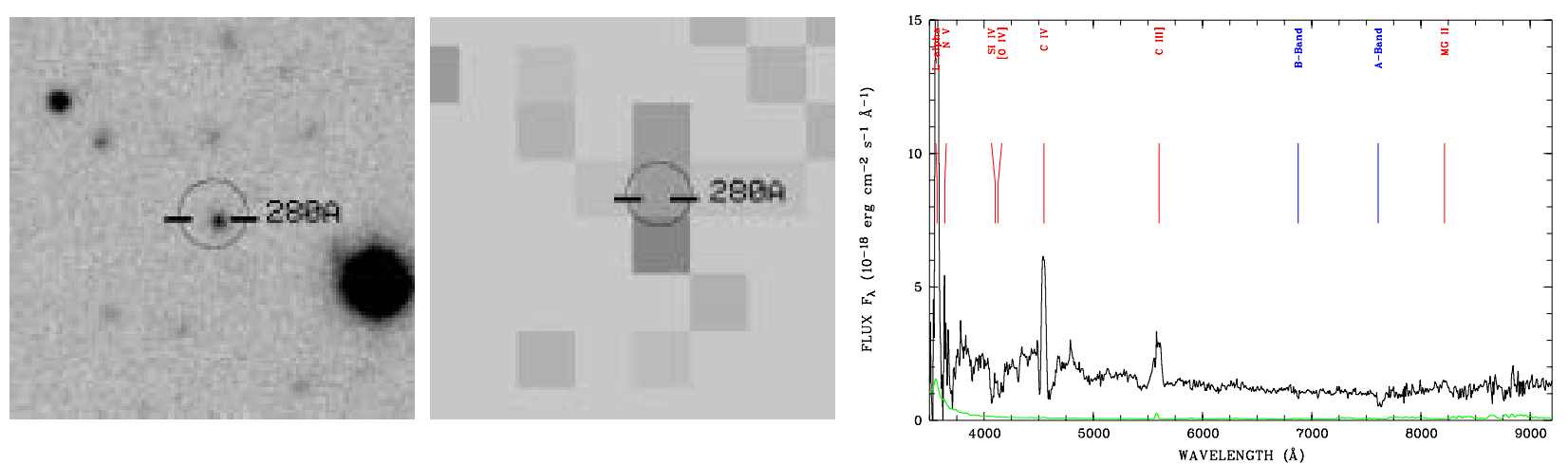

object $280 \mathrm{~A}, \mathrm{z}=1.936$ 
M. Krumpe et al.: The XMM-Newton survey in the Marano field. I., Online Material p 44
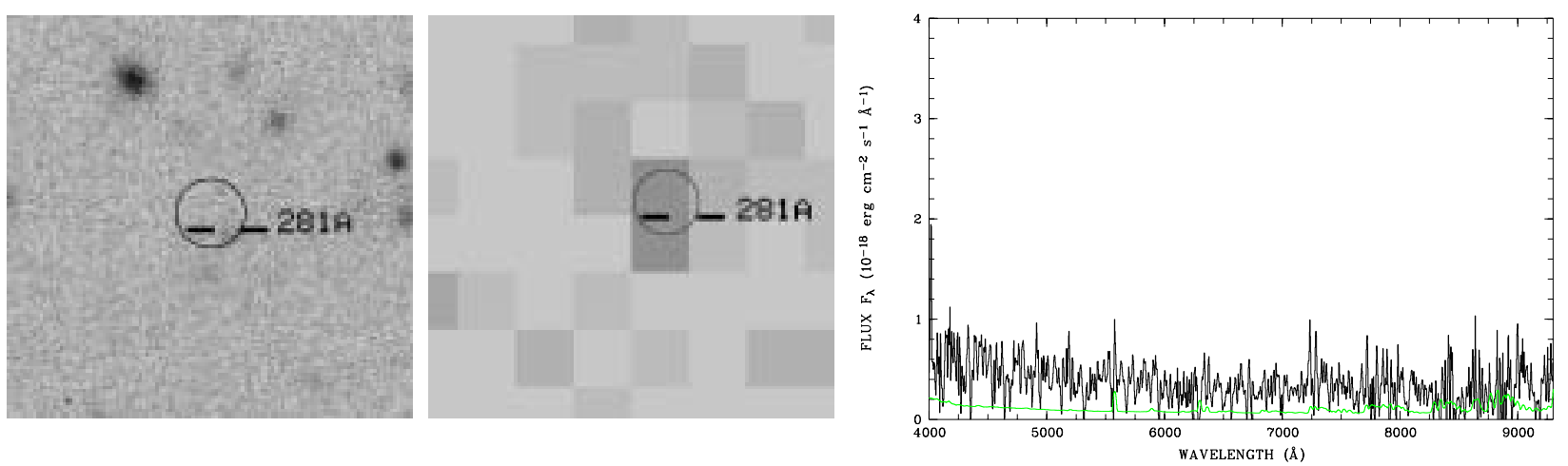

object 281A, no redshift, spectrum too noisy
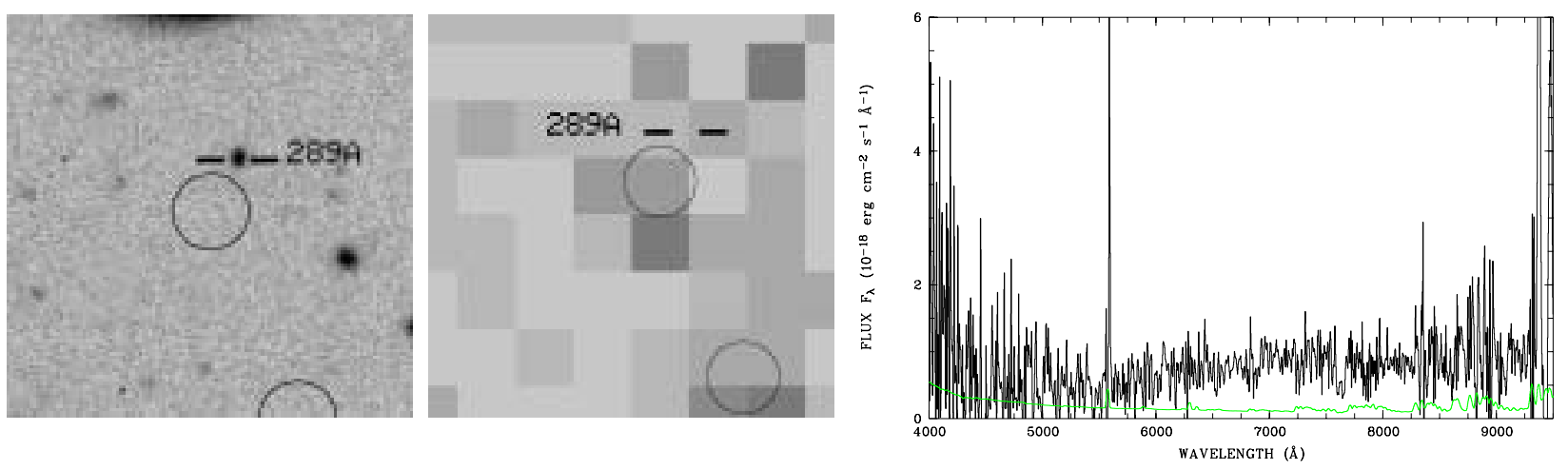

object 289A, no redshift, spectrum too noisy
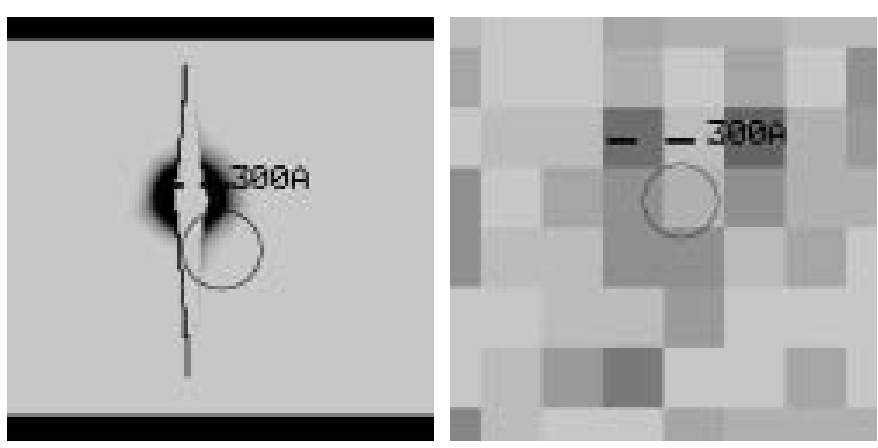

object $300 \mathrm{~A}, \mathrm{z}=0.000$
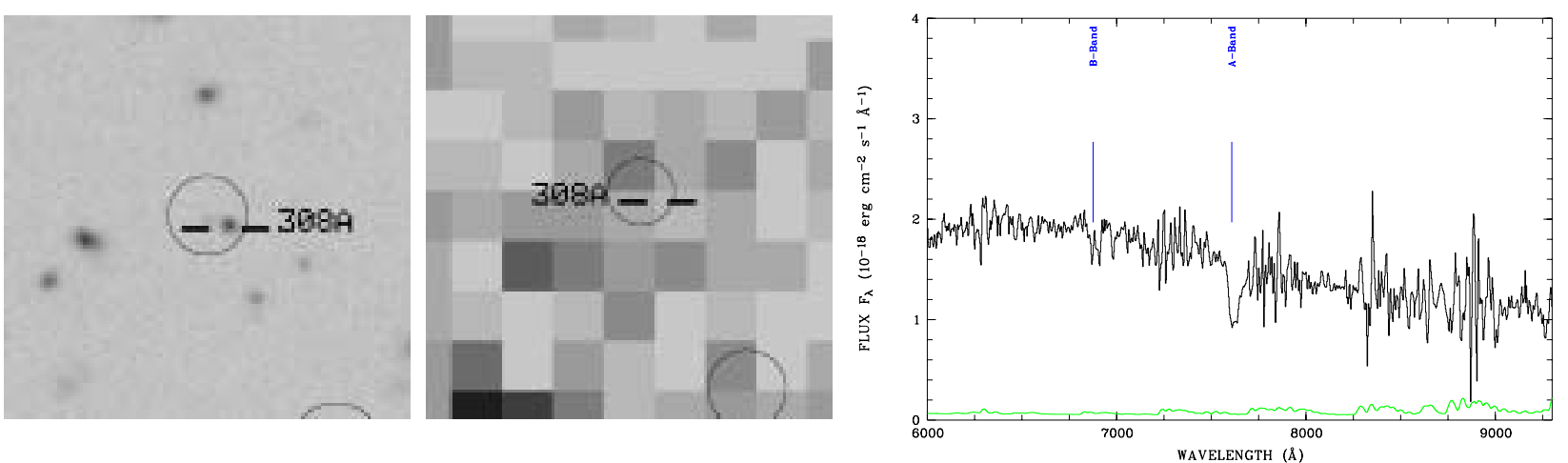

object 308A, no redshift, spectrum inconclusive 
M. Krumpe et al.: The XMM-Newton survey in the Marano field. I., Online Material p 45
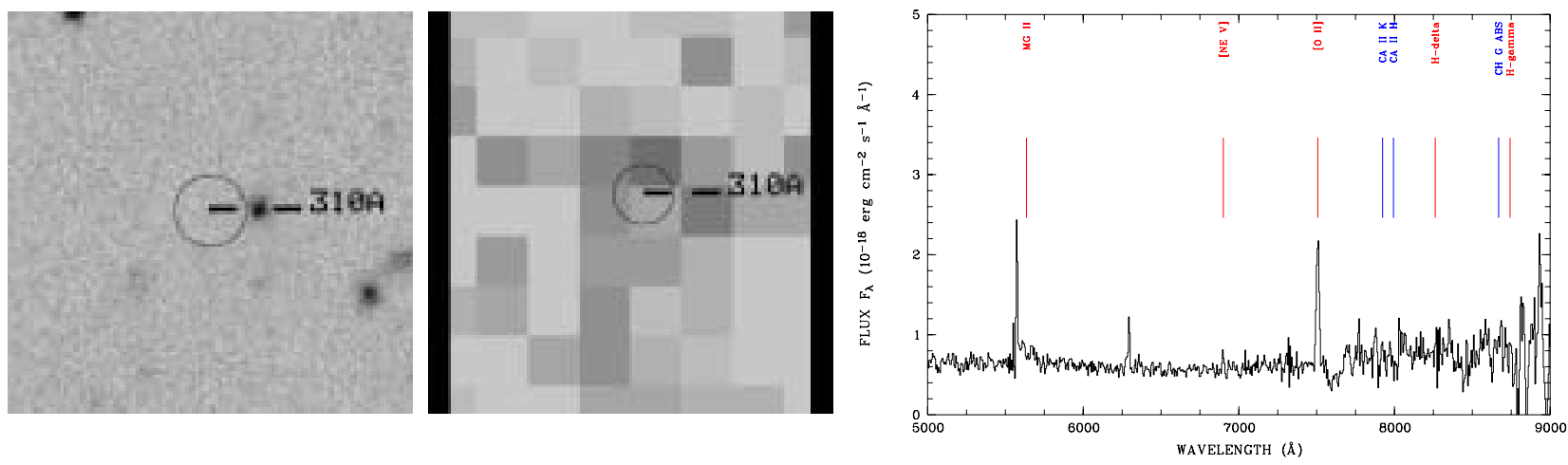

object $310 \mathrm{~A}, \mathrm{z}=1.01$
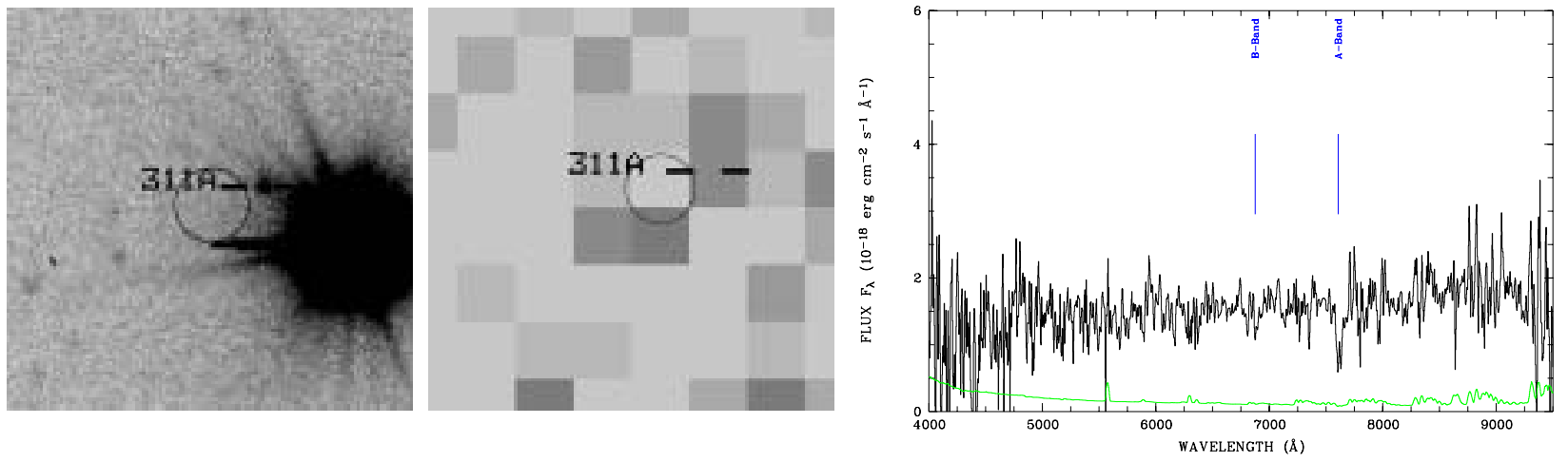

object 311A, no redshift, spectrum too noisy
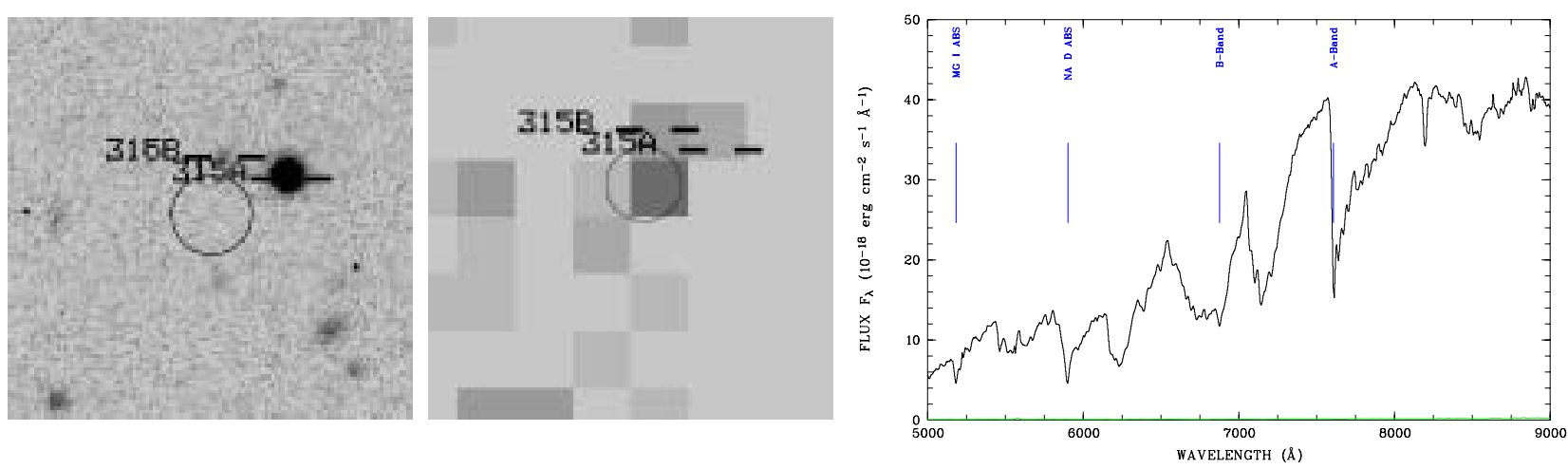

object $315 \mathrm{~A}, \mathrm{z}=0.000$
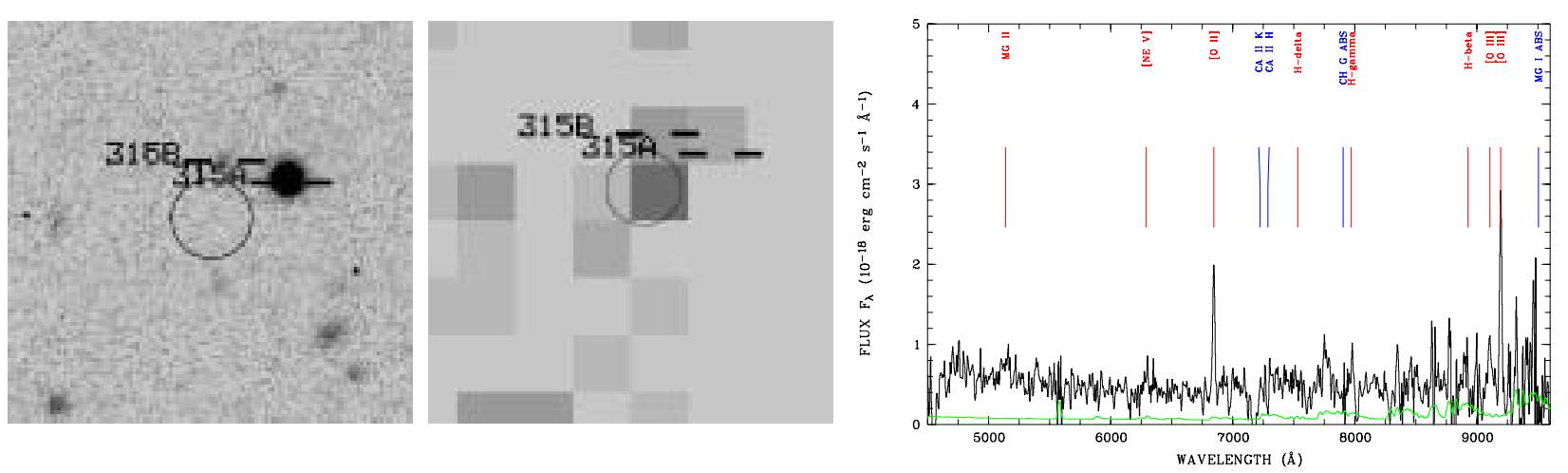

object $315 \mathrm{~B}, \mathrm{z}=0.836$ 
M. Krumpe et al.: The XMM-Newton survey in the Marano field. I., Online Material p 46
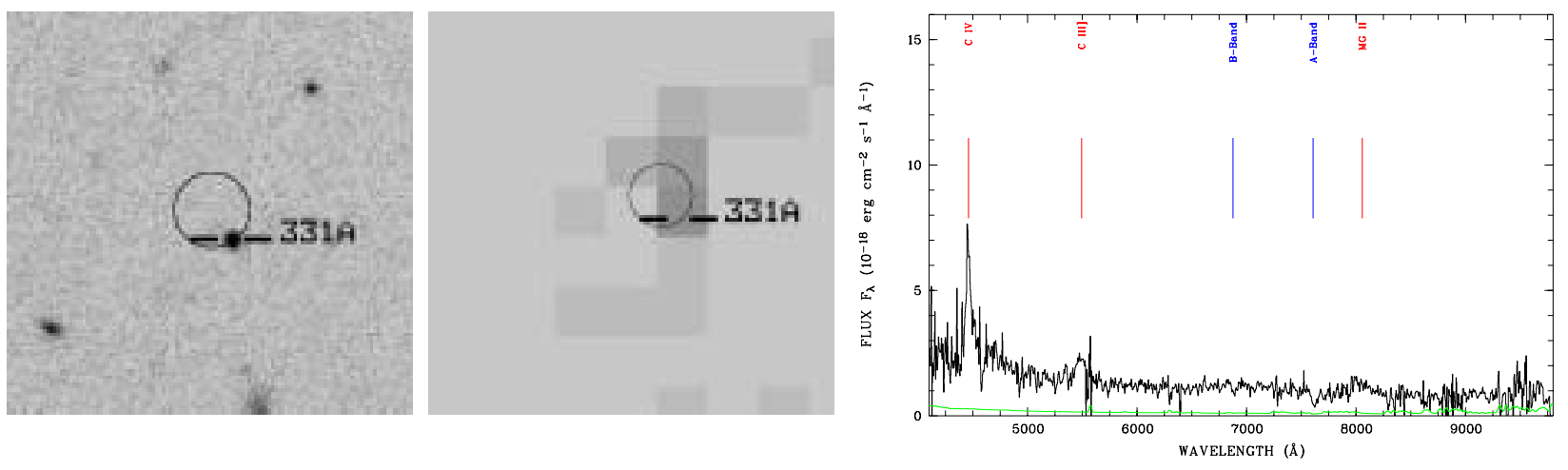

object $331 \mathrm{~A}, \mathrm{z}=1.876$
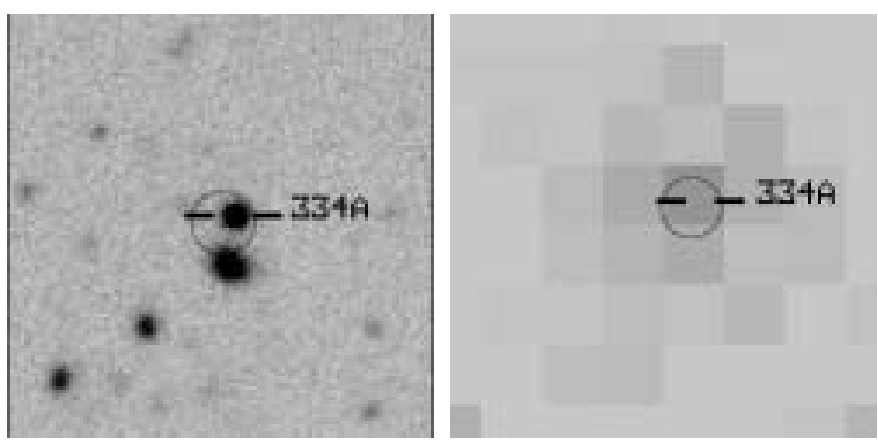

object $334 \mathrm{~A}, \mathrm{z}=2.536$
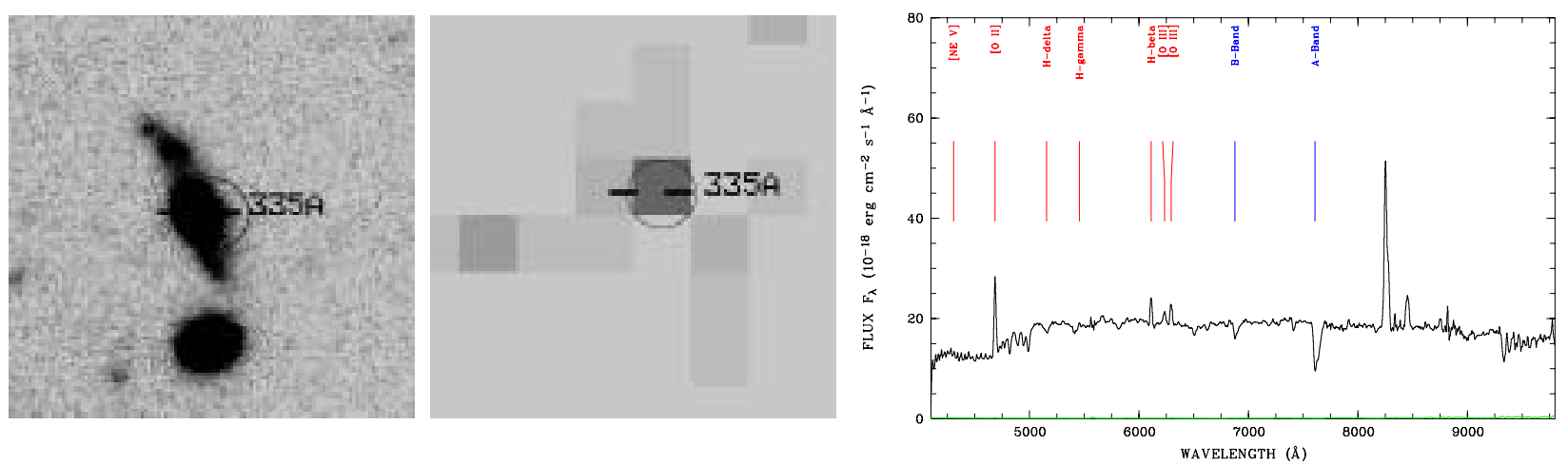

object $335 \mathrm{~A}, \mathrm{z}=0.257$
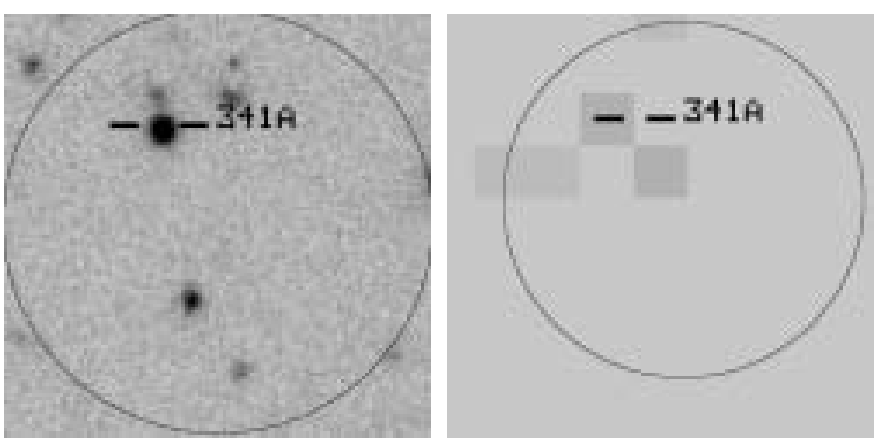

object $341 \mathrm{~A}, \mathrm{z}=2.784$ 
M. Krumpe et al.: The XMM-Newton survey in the Marano field. I., Online Material p 47
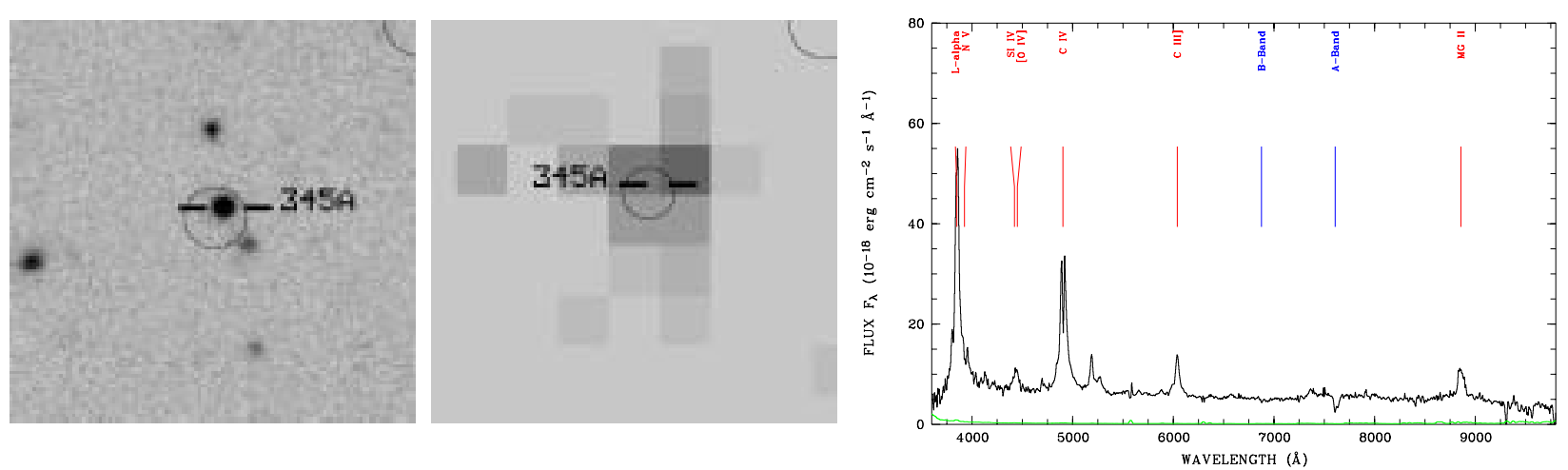

object $345 \mathrm{~A}, \mathrm{z}=2.166$
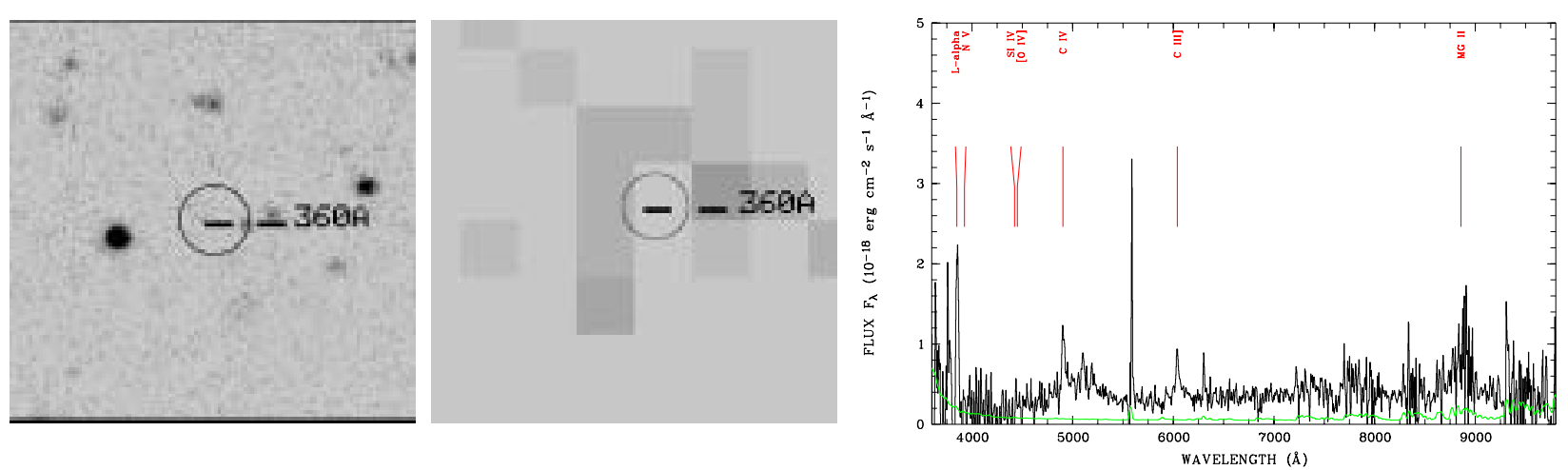

object $360 \mathrm{~A}, \mathrm{z}=2.166$
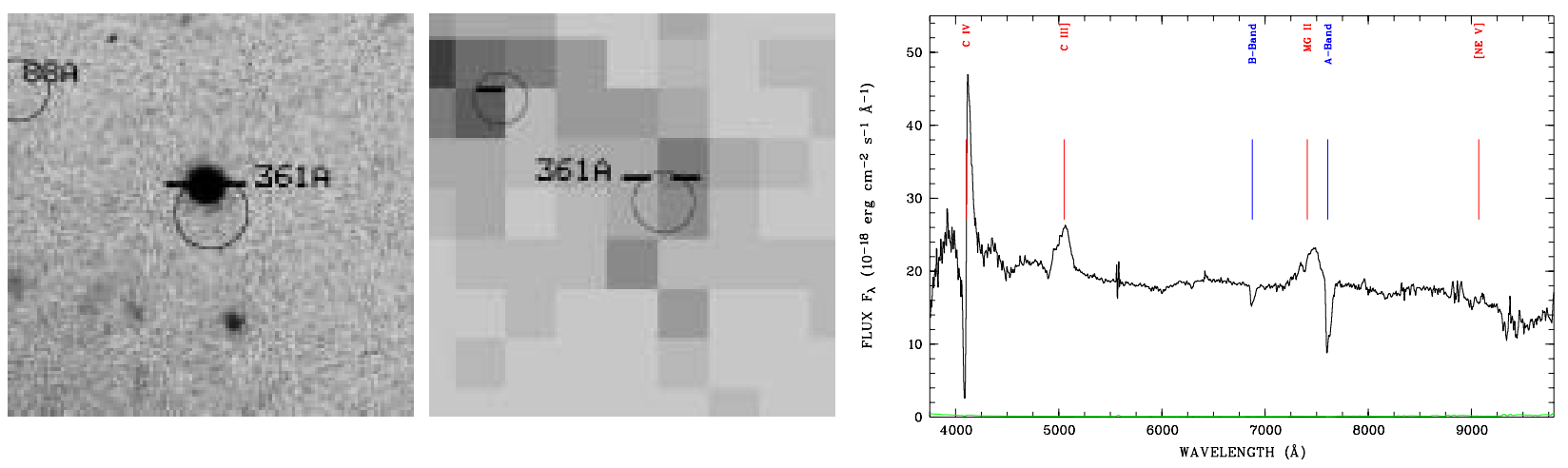

object $361 \mathrm{~A}, \mathrm{z}=1.648$
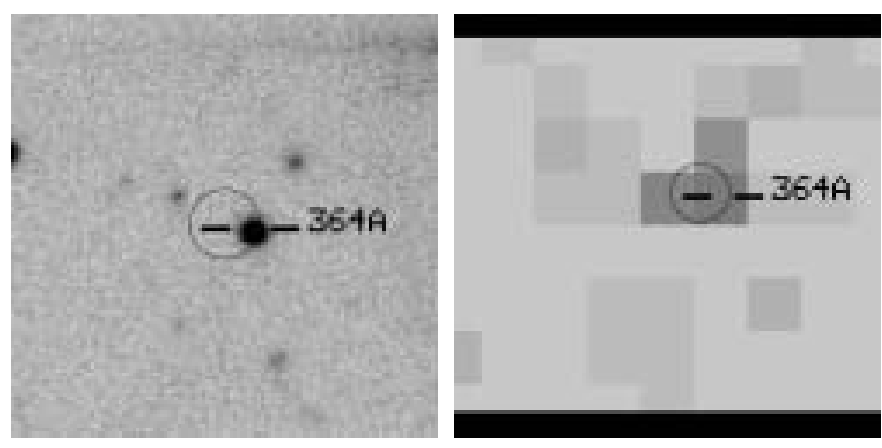

object $364 \mathrm{~A}, \mathrm{z}=1.821$ 

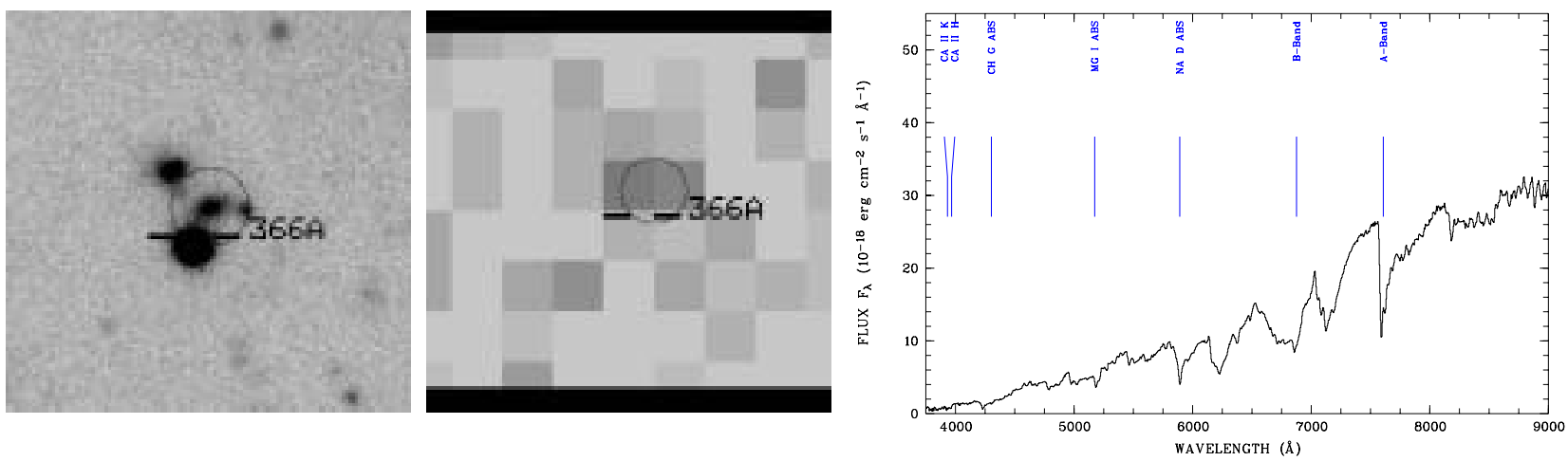

object $366 \mathrm{~A}, \mathrm{z}=0.00$
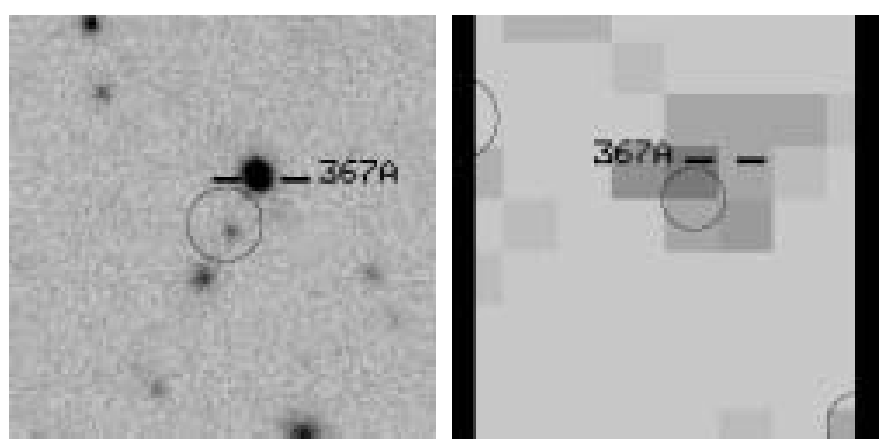

object 367A, z $=0.981$
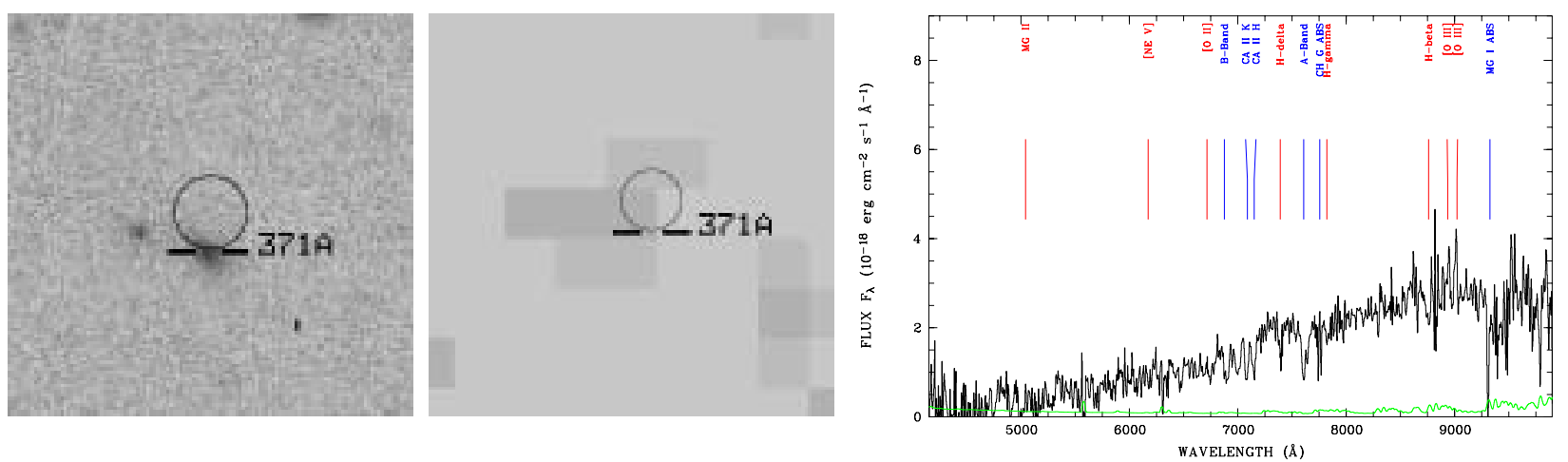

object $371 \mathrm{~A}, \mathrm{z}=0.802$
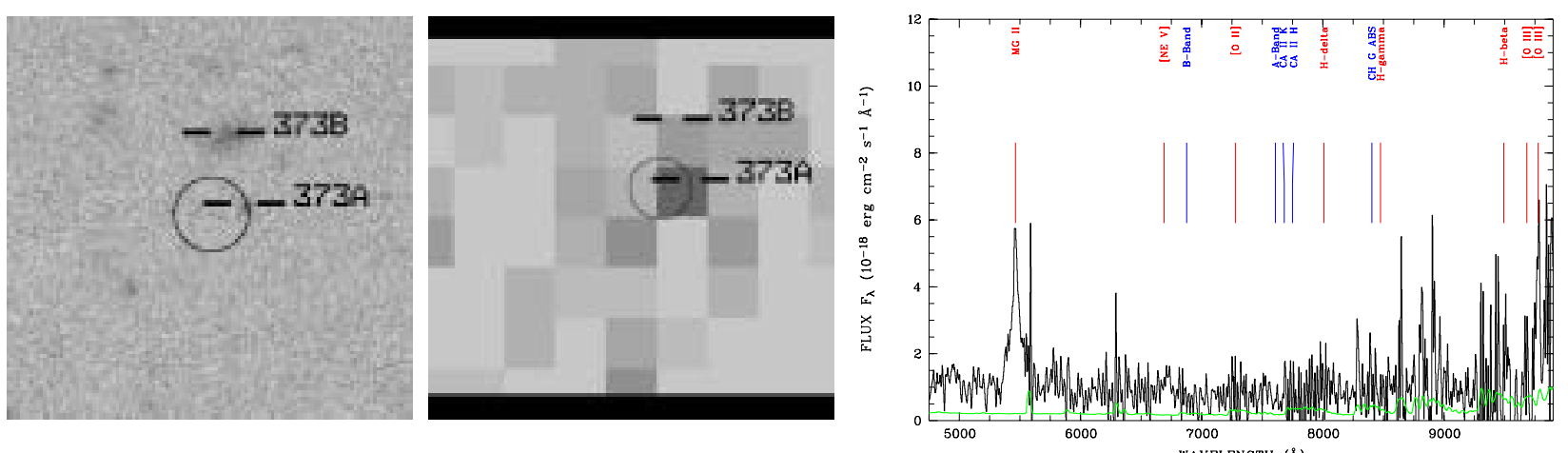

object 373A, z=0.953:@MgII 
M. Krumpe et al.: The XMM-Newton survey in the Marano field. I., Online Material p 49
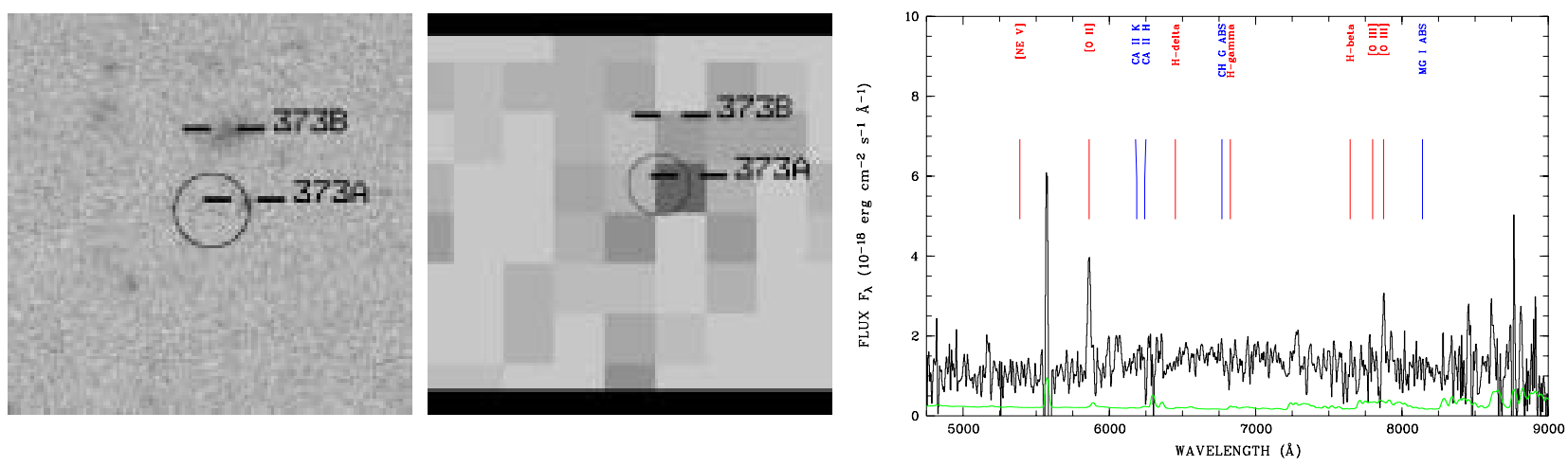

object $373 B, z=0.573$
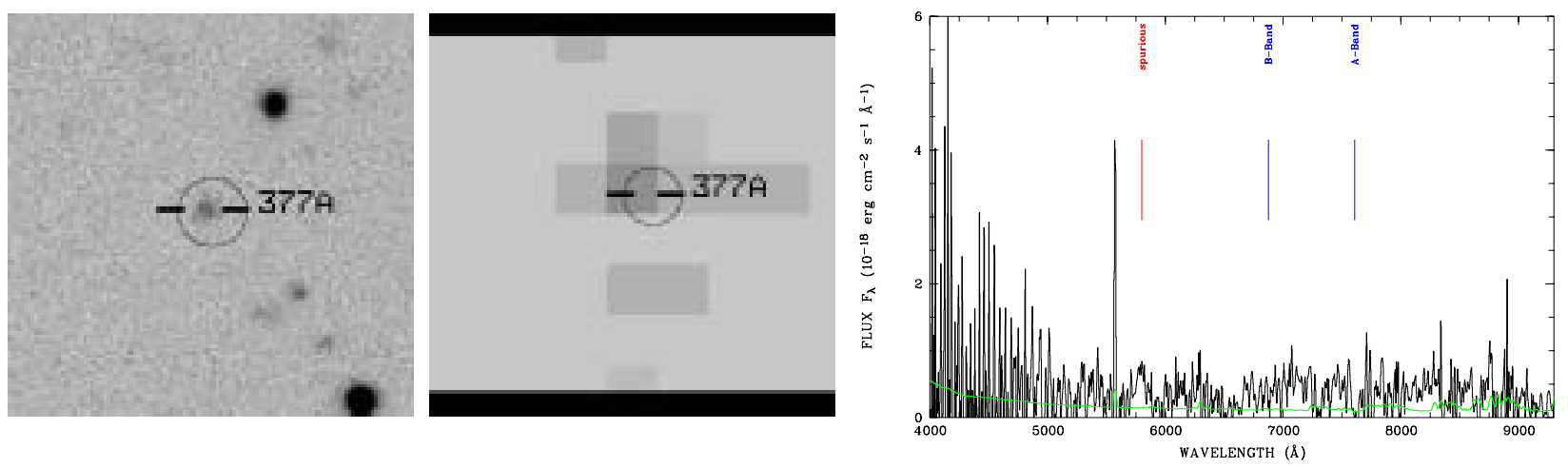

object 377A, no redshift, spectrum too noisy
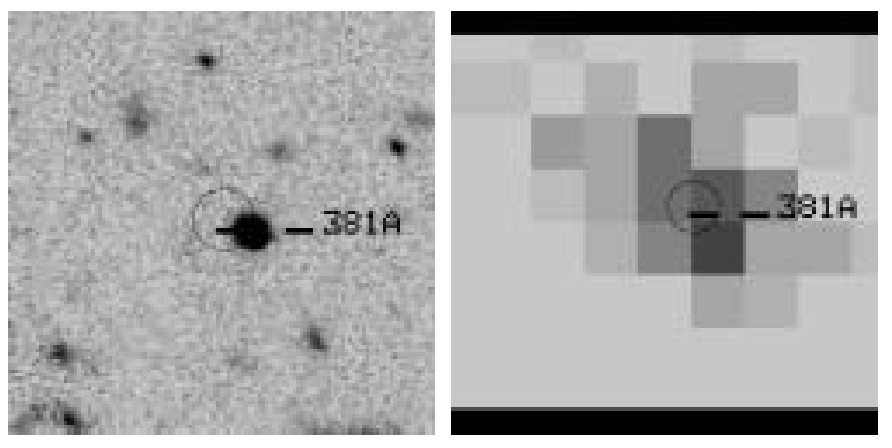

object $381 \mathrm{~A}, \mathrm{z}=1.879$
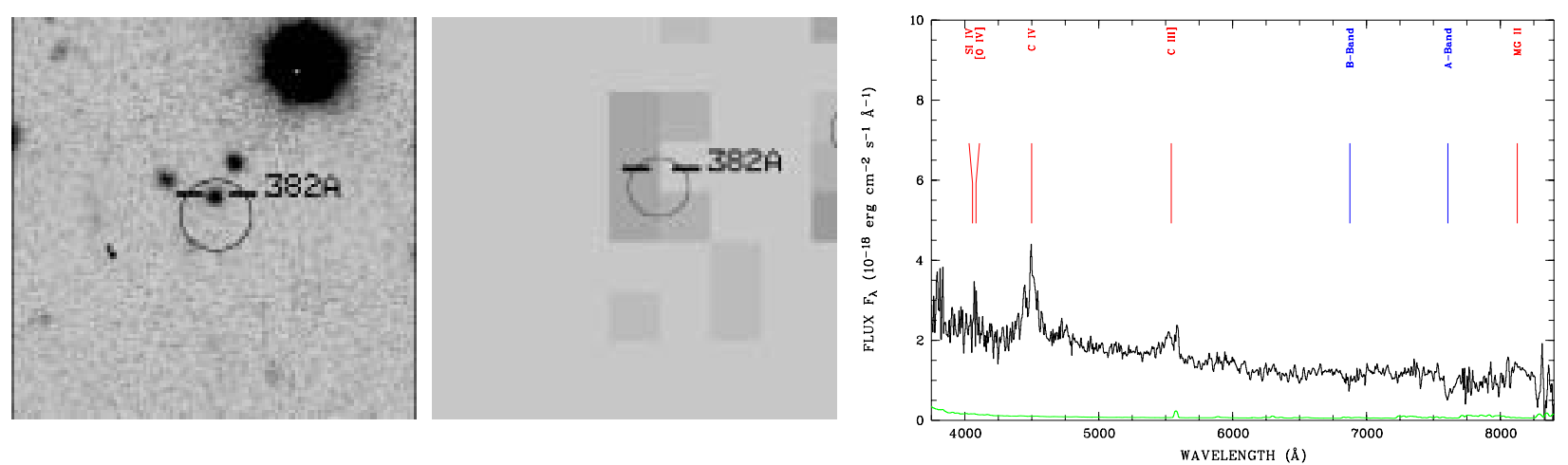

object $382 \mathrm{~A}, \mathrm{z}=1.904$ 
M. Krumpe et al.: The XMM-Newton survey in the Marano field. I., Online Material p 50
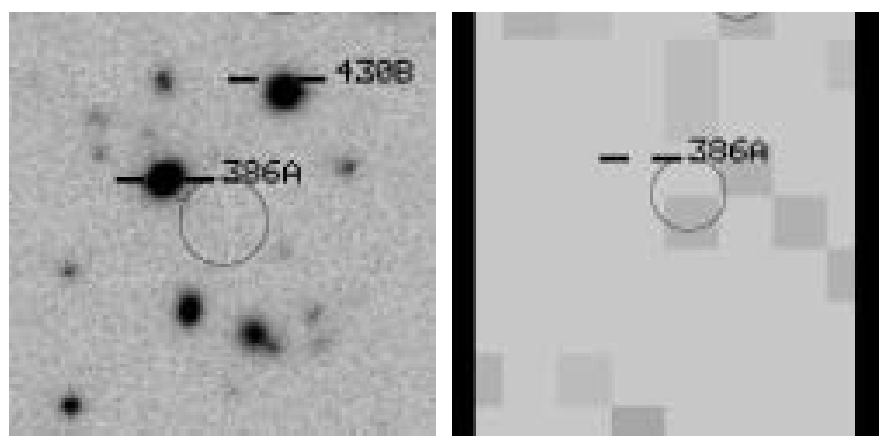

object $386 \mathrm{~A}, \mathrm{z}=0.189$
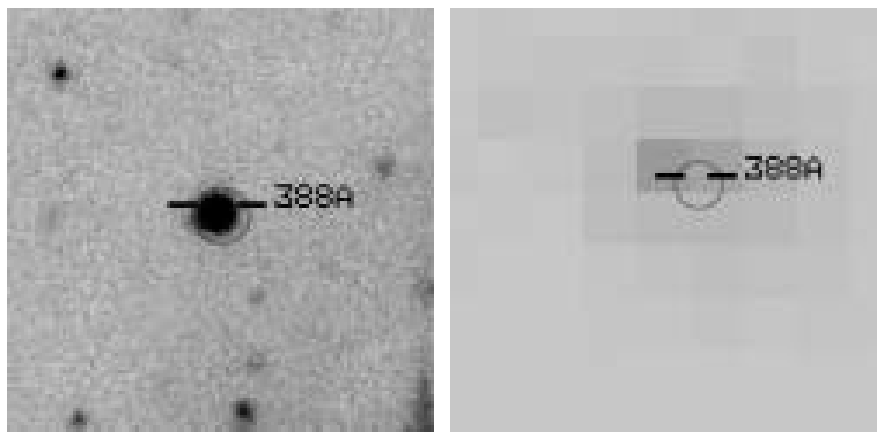

object $388 \mathrm{~A}, \mathrm{z}=0.841$
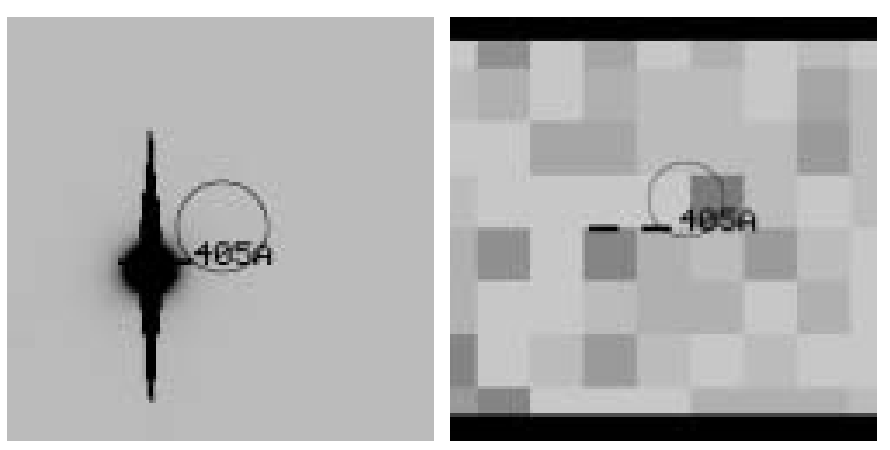

object $405 \mathrm{~A}, \mathrm{z}=0.000$
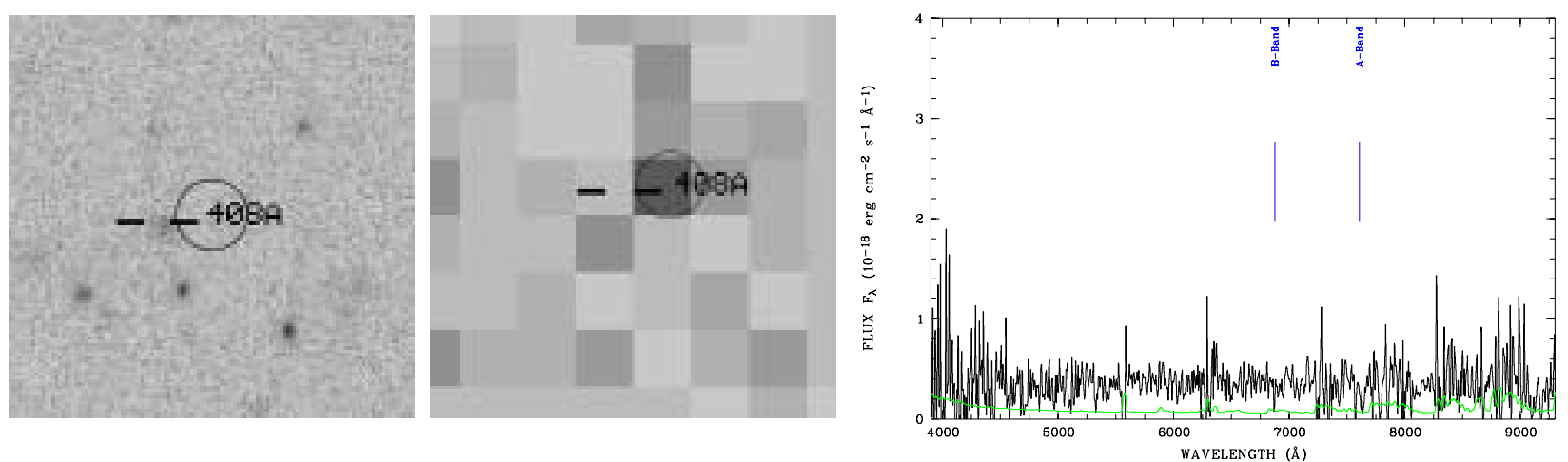

object 408A, no redshift, spectrum too noisy 
M. Krumpe et al.: The XMM-Newton survey in the Marano field. I., Online Material p 51

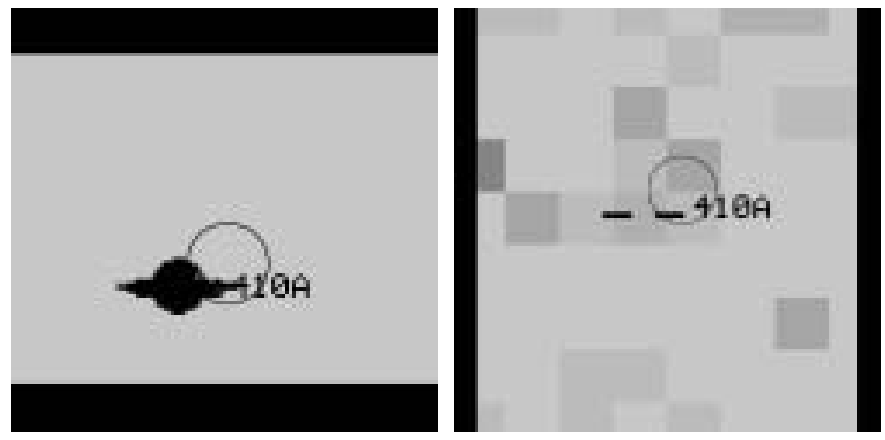

object $410 \mathrm{~A}, \mathrm{z}=0.000$
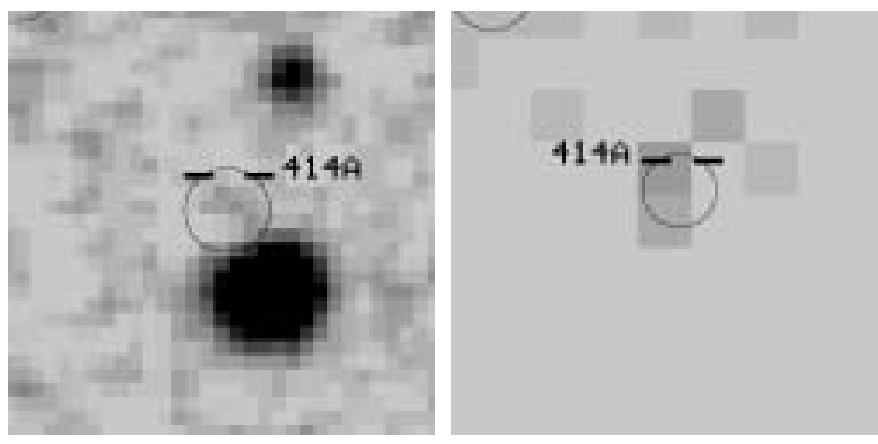

object $414 \mathrm{~A}, \mathrm{z}=2.058$
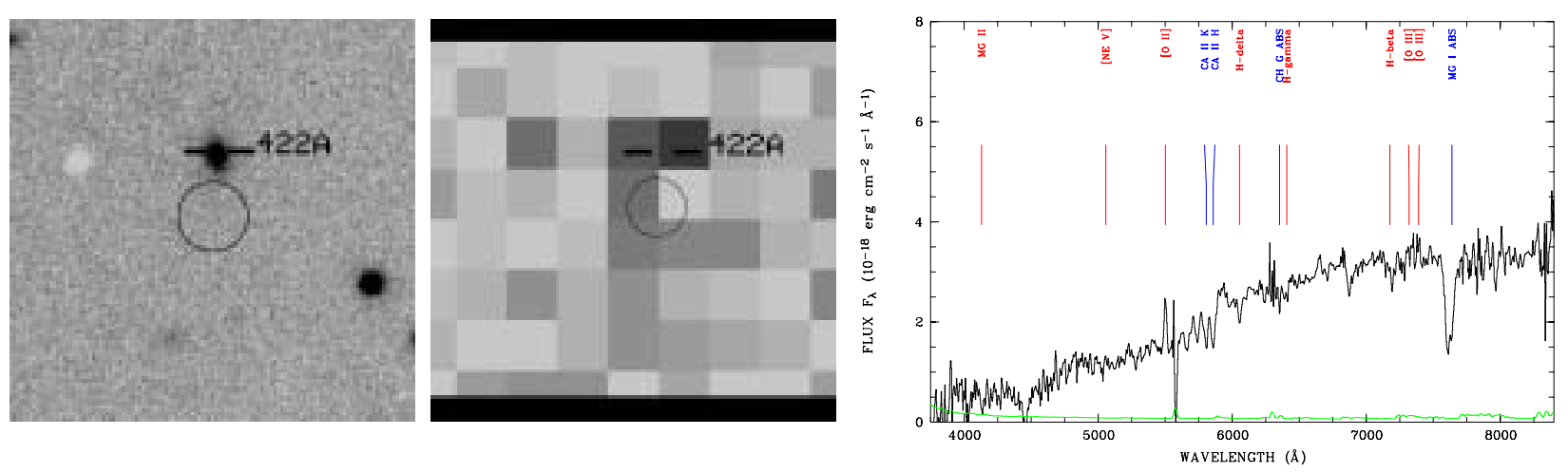

object $422 \mathrm{~A}, \mathrm{z}=0.476$
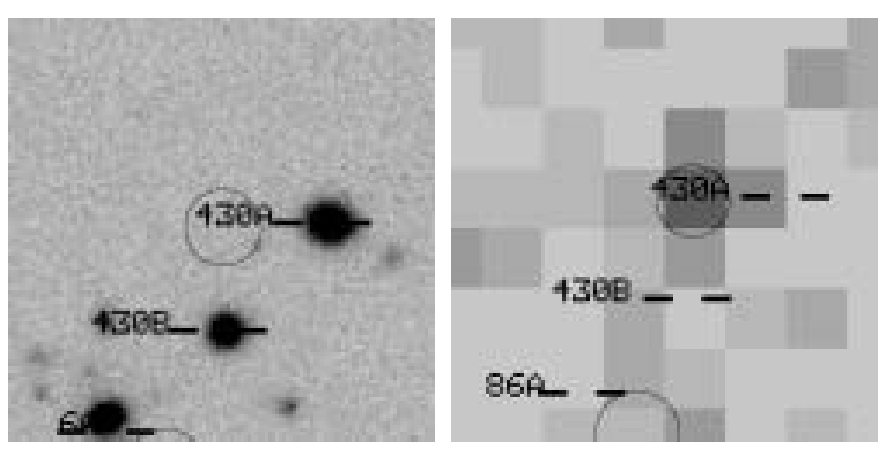

object $430 \mathrm{~A}, \mathrm{z}=0.390$ 

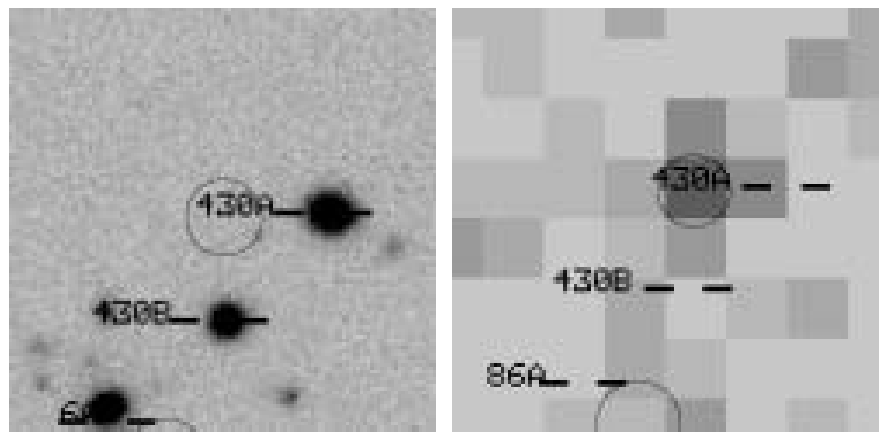

object $430 \mathrm{~B}, \mathrm{z}=0.391$
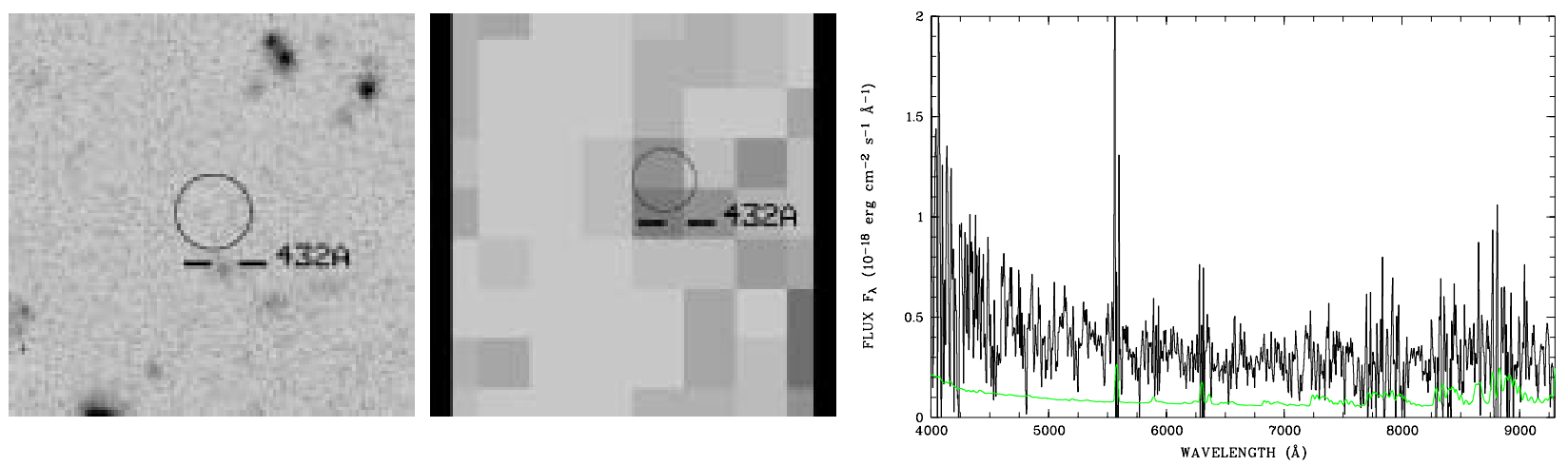

object 432A, no redshift, spectrum too noisy
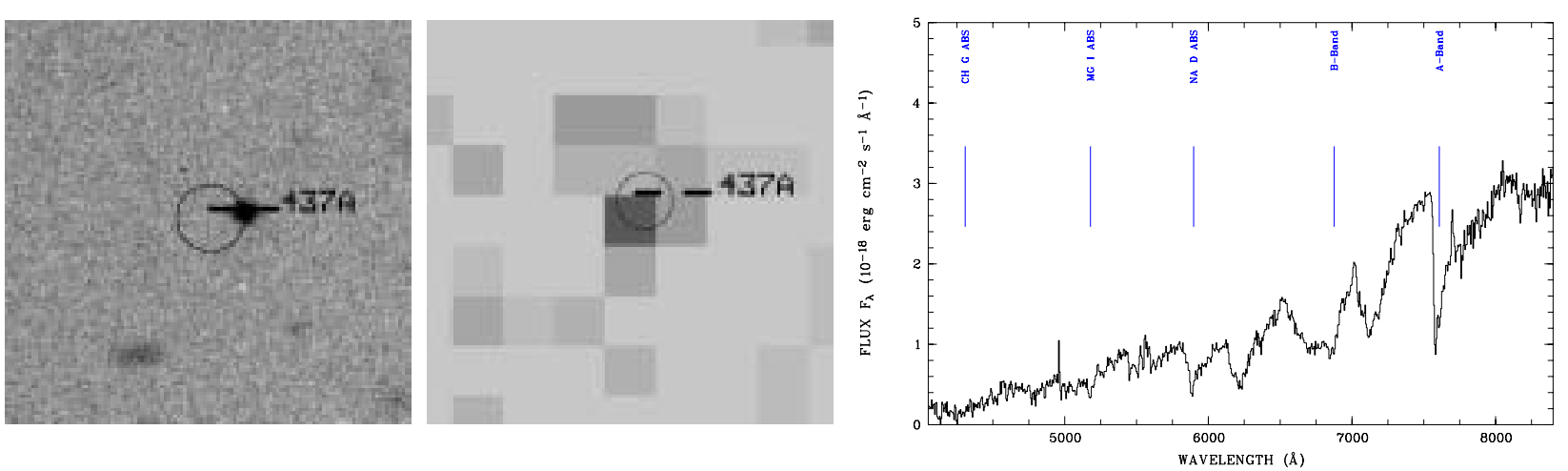

object $437 \mathrm{~A}, \mathrm{z}=0.00$
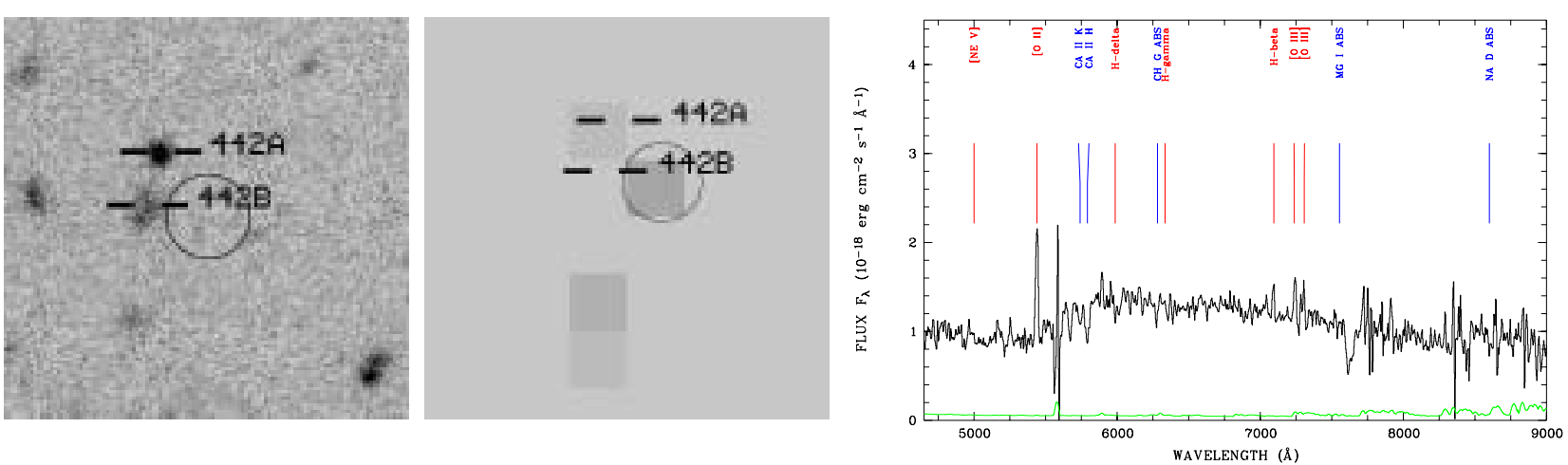

object $442 \mathrm{~A}, \mathrm{z}=0.460$ 
M. Krumpe et al.: The XMM-Newton survey in the Marano field. I., Online Material p 53
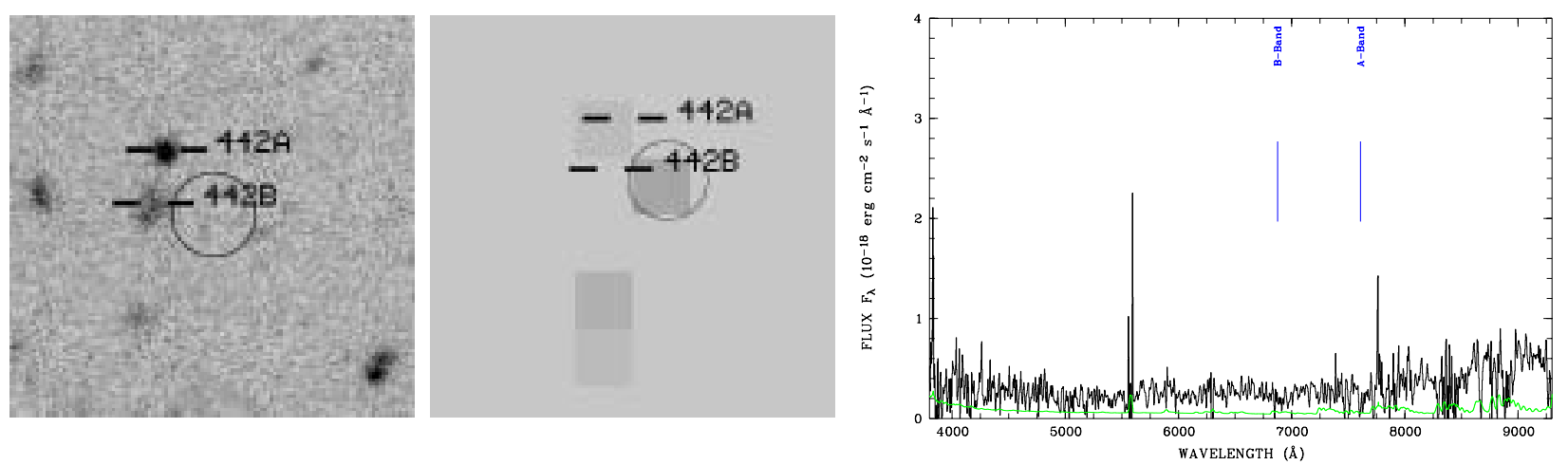

object 442B, no redshift, spectrum too noisy
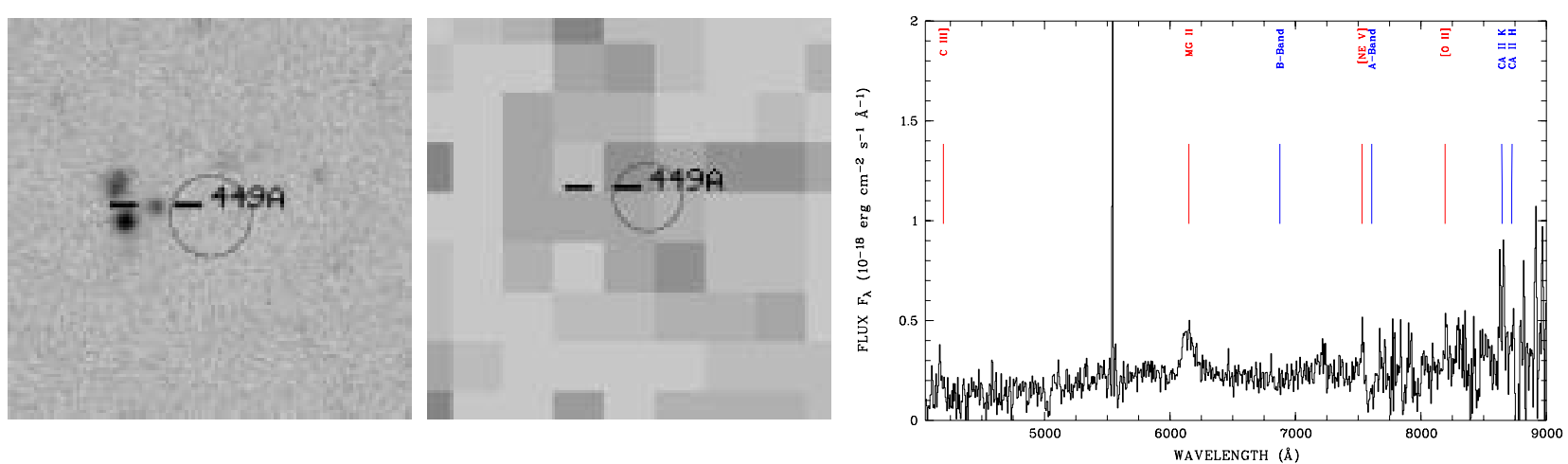

object $449 \mathrm{~A}, \mathrm{z}=1.204$
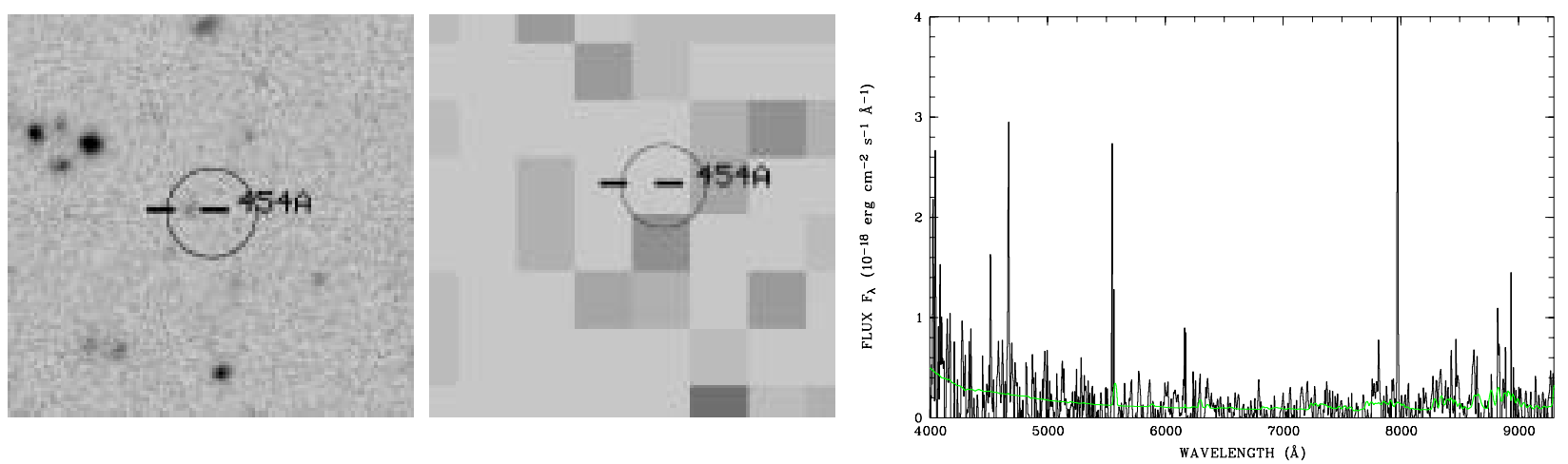

object 454A, no redshift, spectrum too noisy
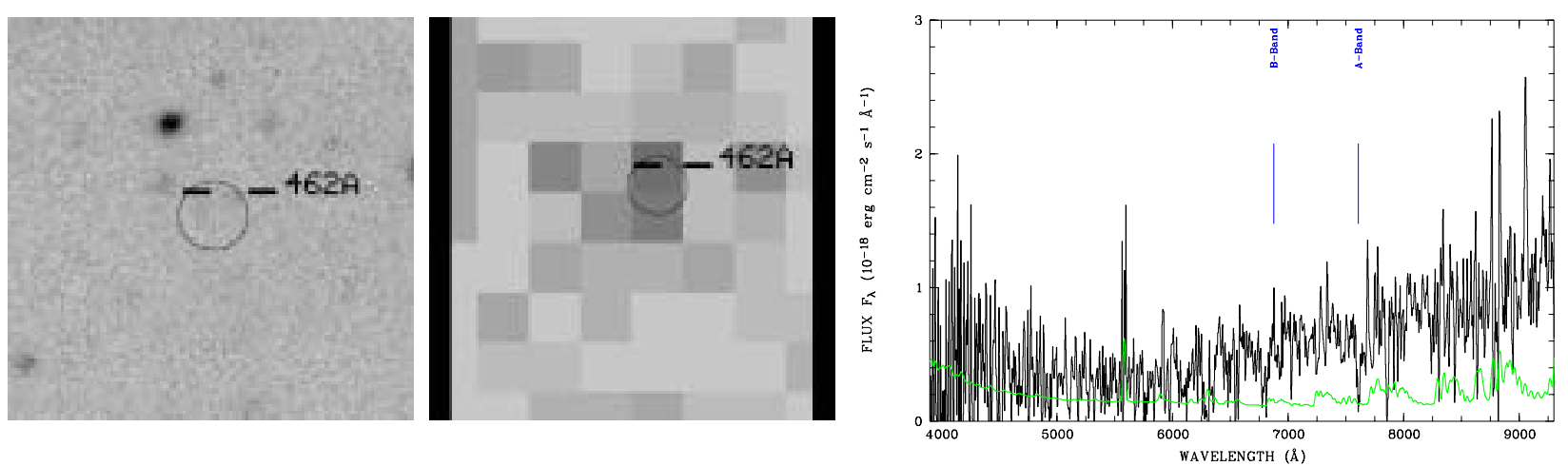

object 462A, no redshift, spectrum too noisy 
M. Krumpe et al.: The XMM-Newton survey in the Marano field. I., Online Material p 54
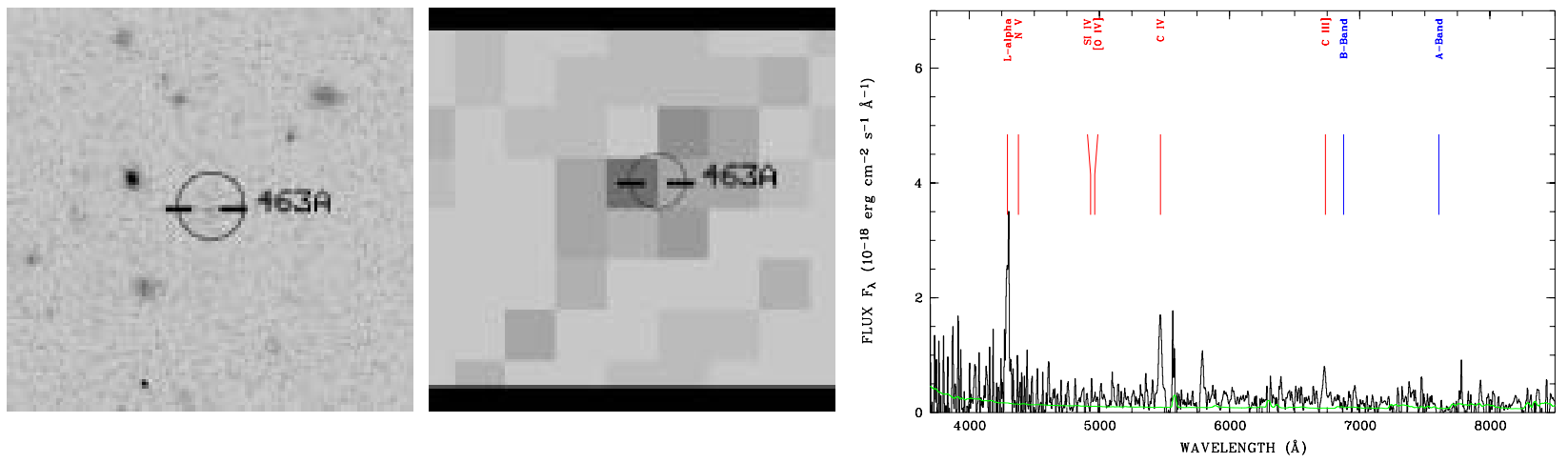

object $463 \mathrm{~A}, \mathrm{z}=2.531$
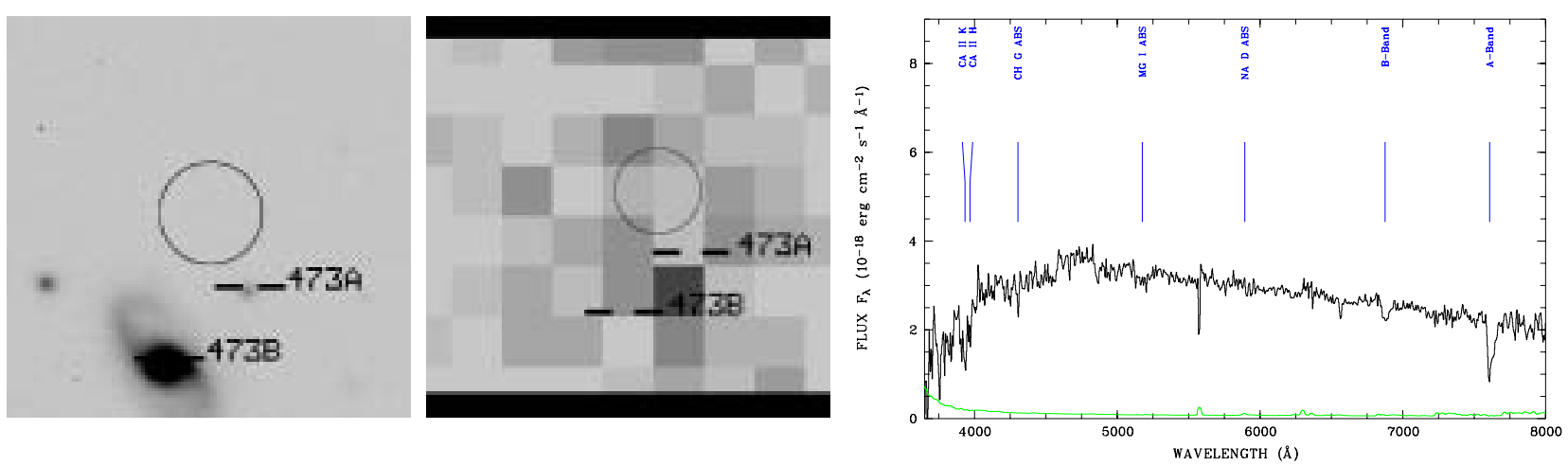

object $473 \mathrm{~A}, \mathrm{z}=0.000$
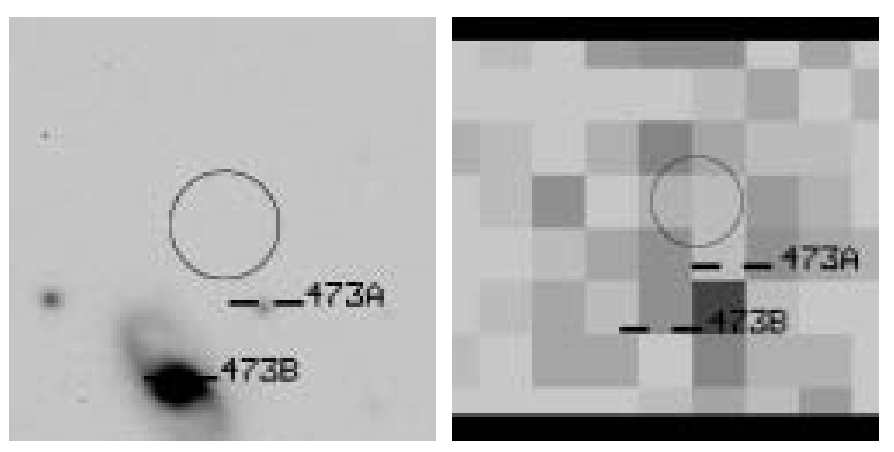

object $473 \mathrm{~B}, \mathrm{z}=0.154$
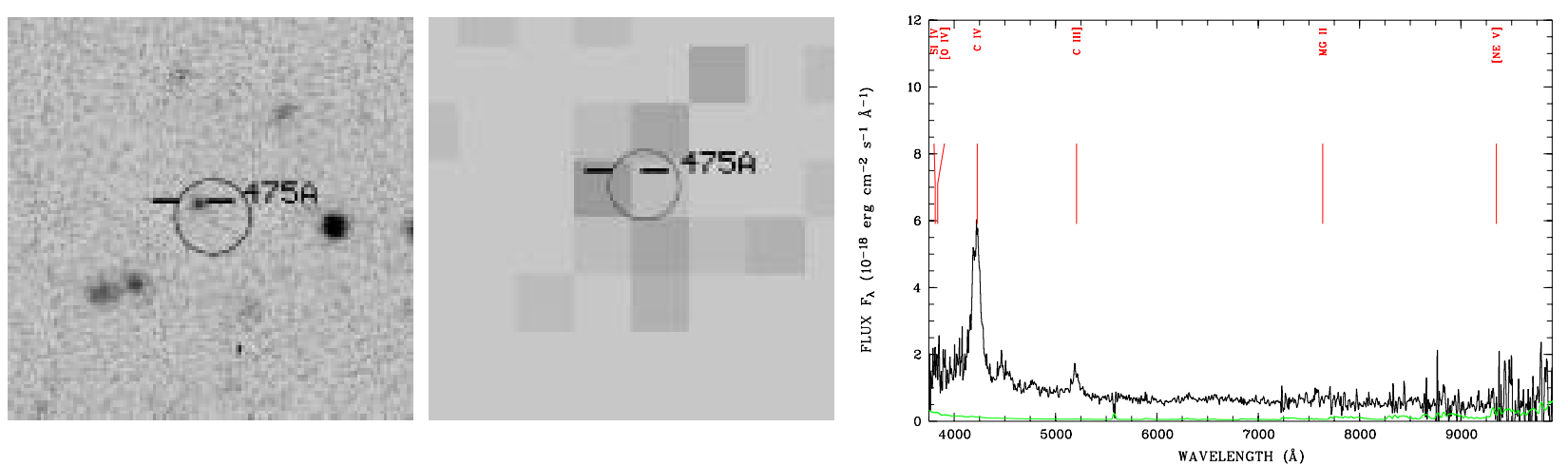

object $475 \mathrm{~A}, \mathrm{z}=1.729$ 
M. Krumpe et al.: The XMM-Newton survey in the Marano field. I., Online Material p 55
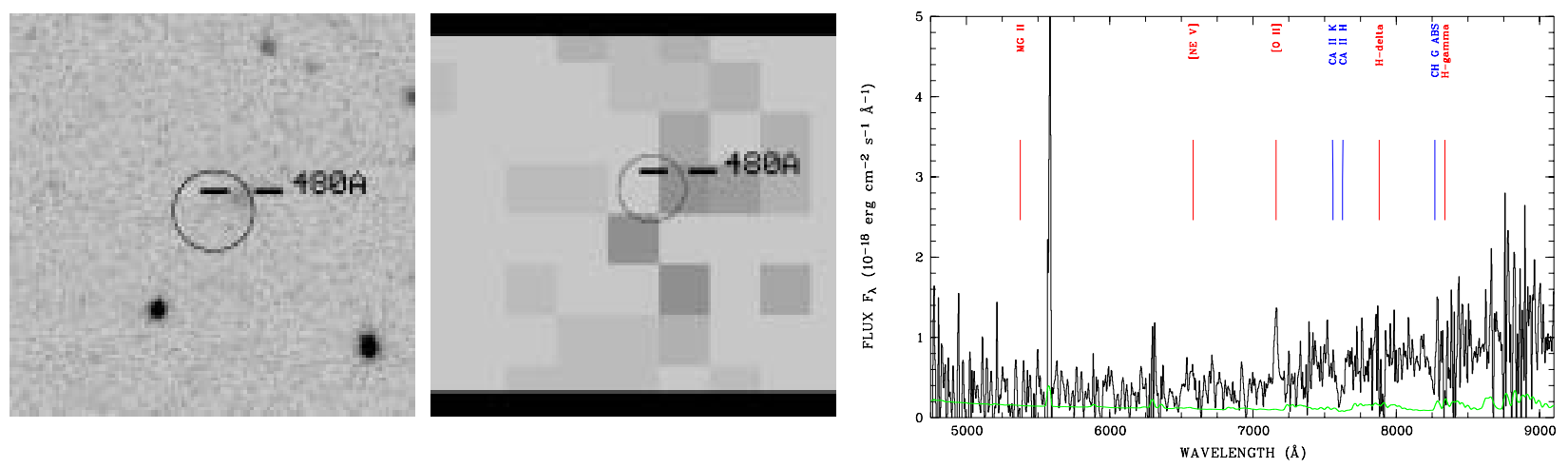

object 480A, z=0.921:@OII
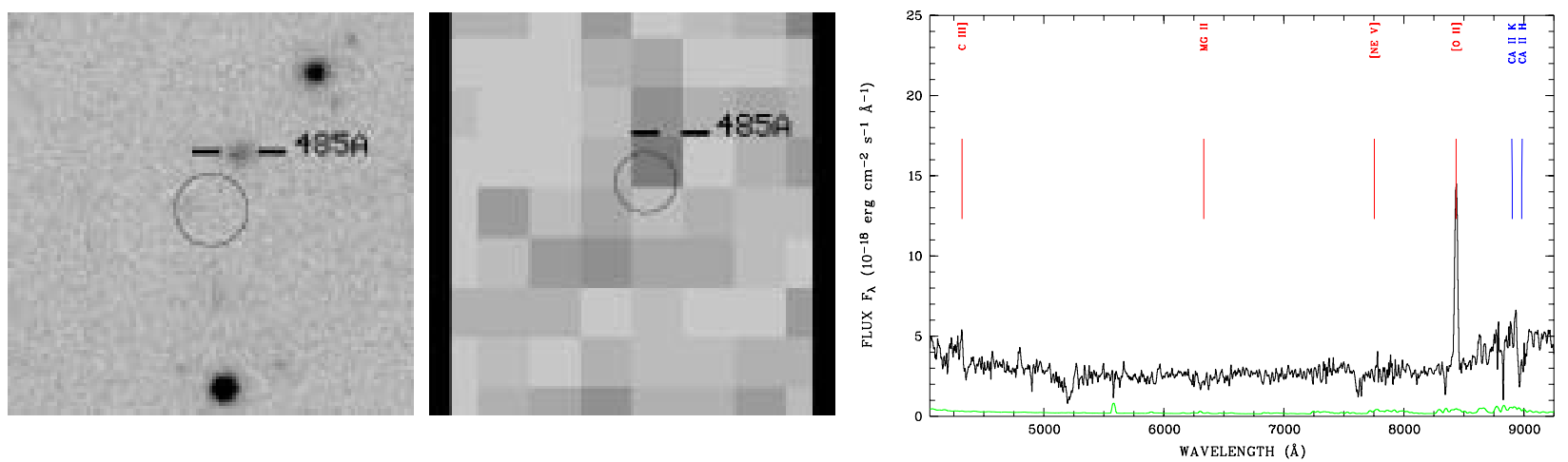

object 485A, z=1.263:@OII
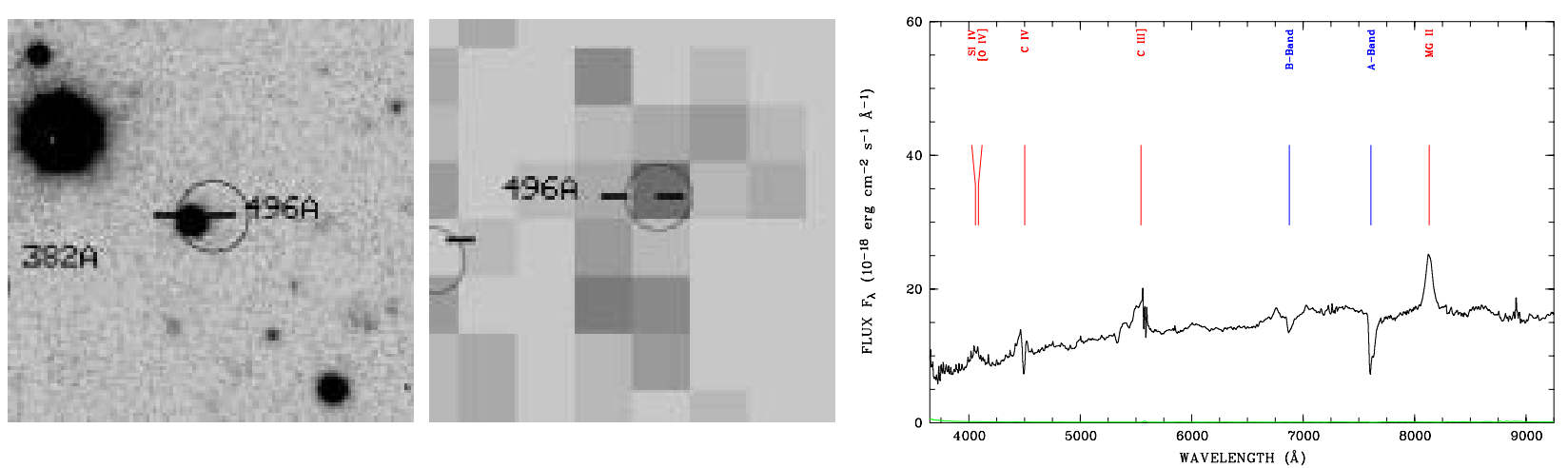

object $496 \mathrm{~A}, \mathrm{z}=1.906$
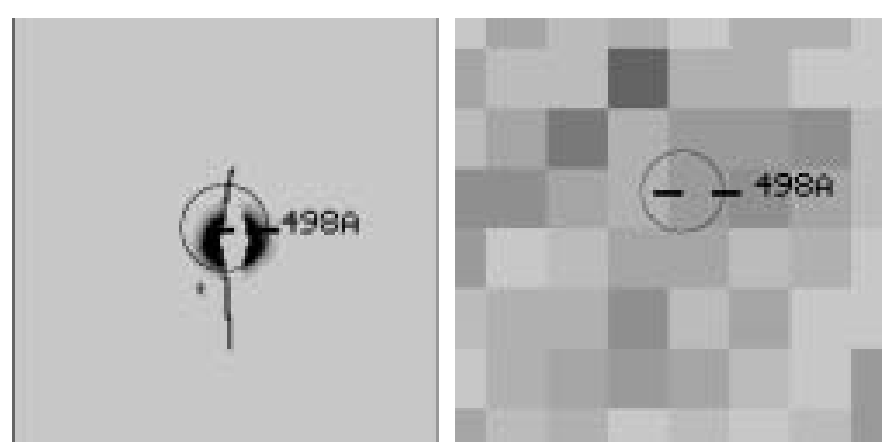

object 498A, $\mathrm{z}=0.00$ 
M. Krumpe et al.: The XMM-Newton survey in the Marano field. I., Online Material p 56
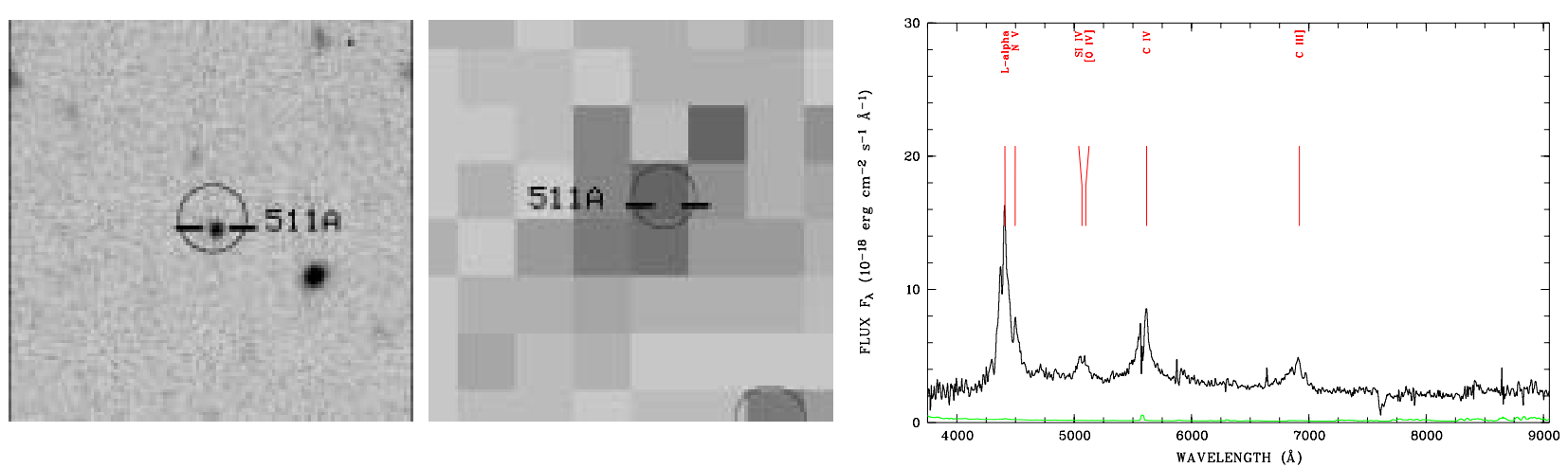

object $511 \mathrm{~A}, \mathrm{z}=2.623$
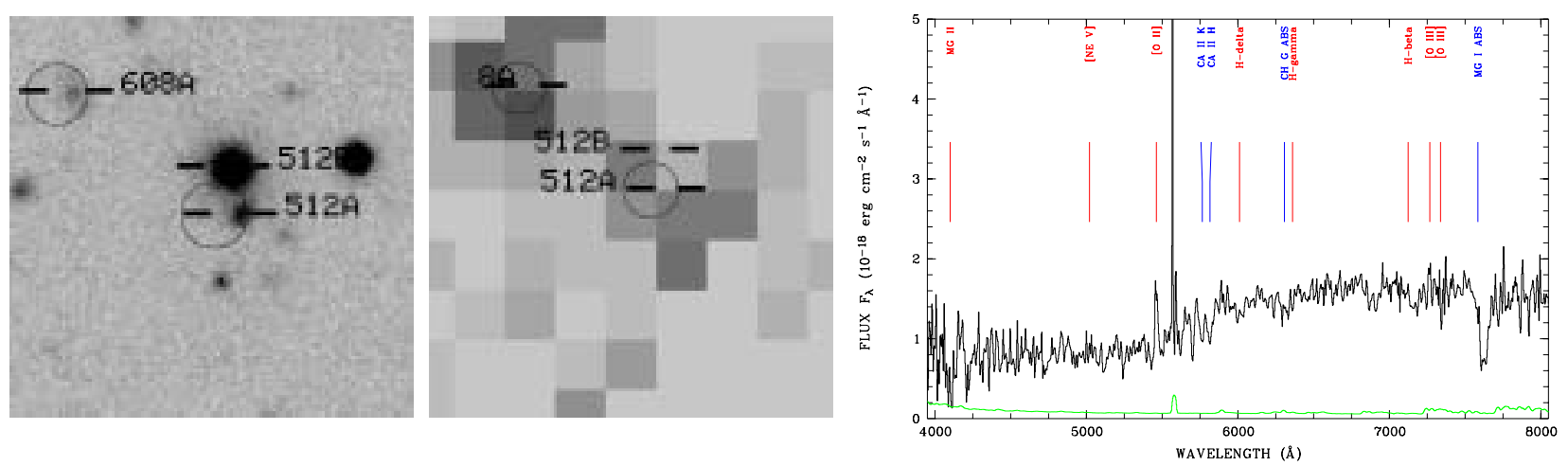

object $512 \mathrm{~A}, \mathrm{z}=0.465$
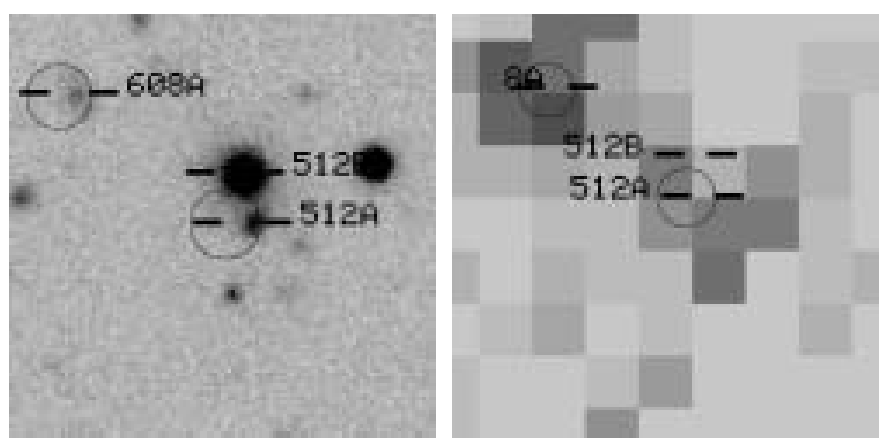

object $512 \mathrm{~B}, \mathrm{z}=0.584$
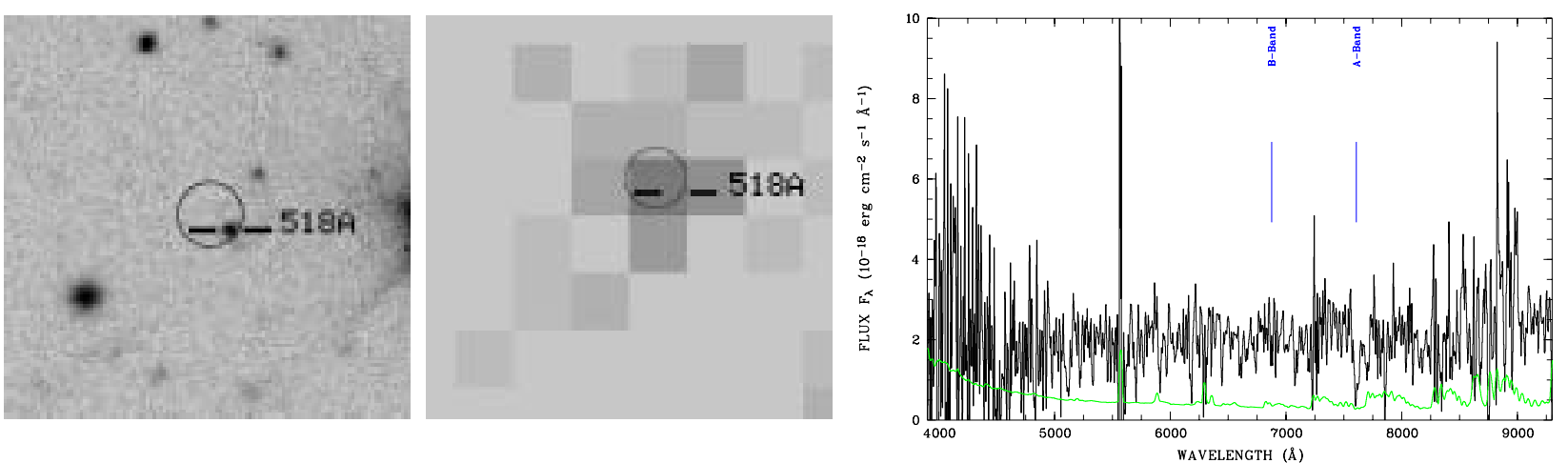

object 518A, no redshift, spectrum too noisy 
M. Krumpe et al.: The XMM-Newton survey in the Marano field. I., Online Material p 57
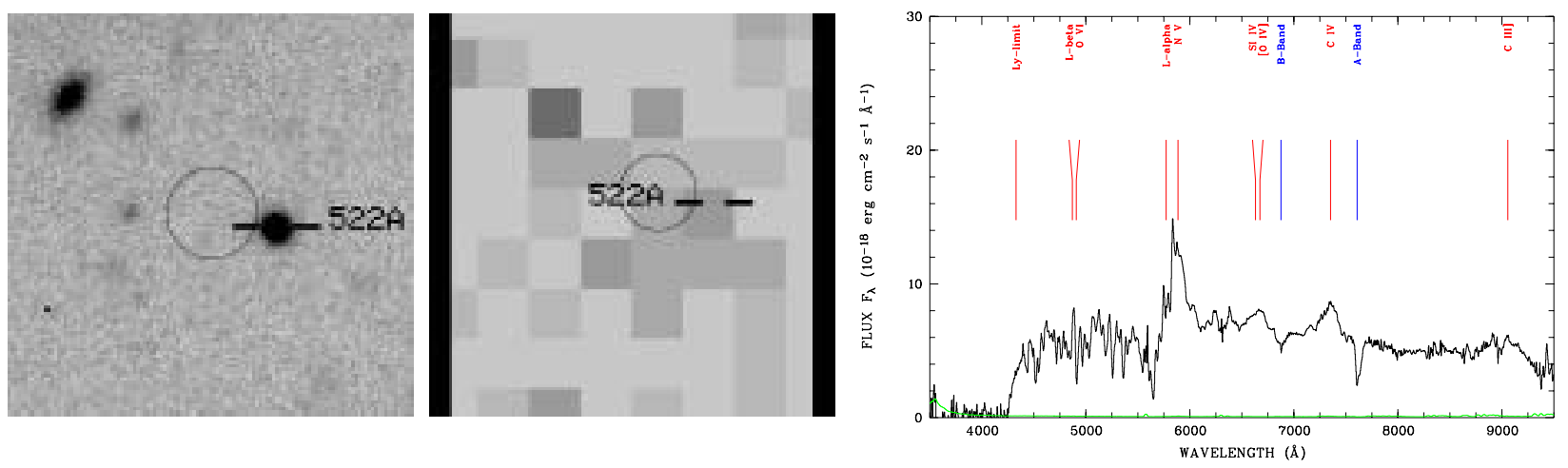

object $522 \mathrm{~A}, \mathrm{z}=3.747$
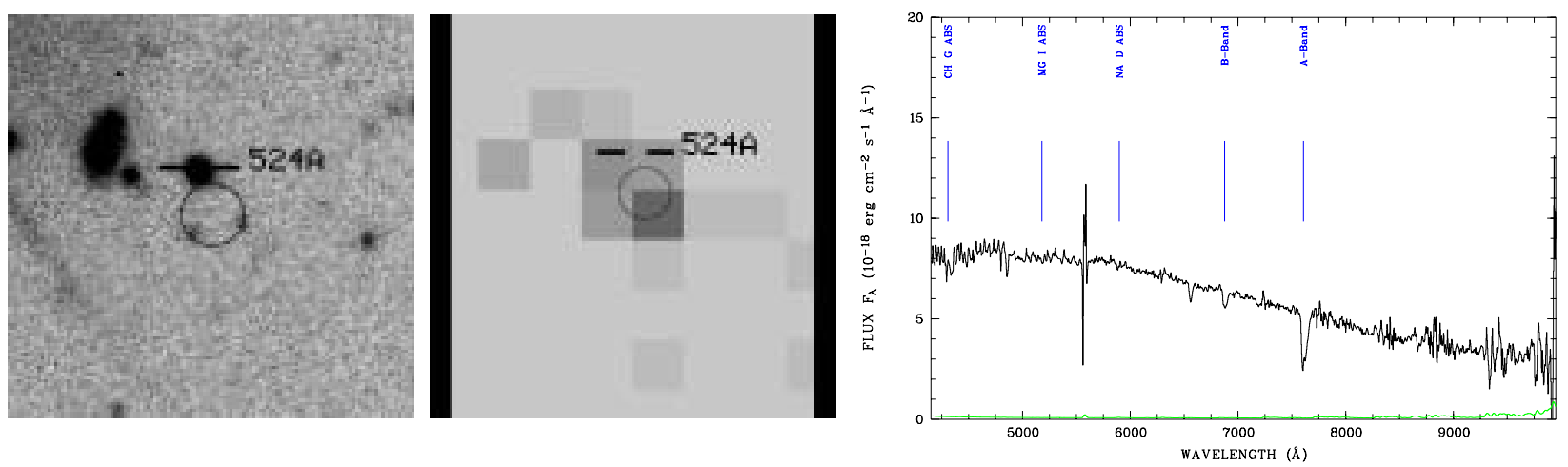

object 524A, z $=0.000$
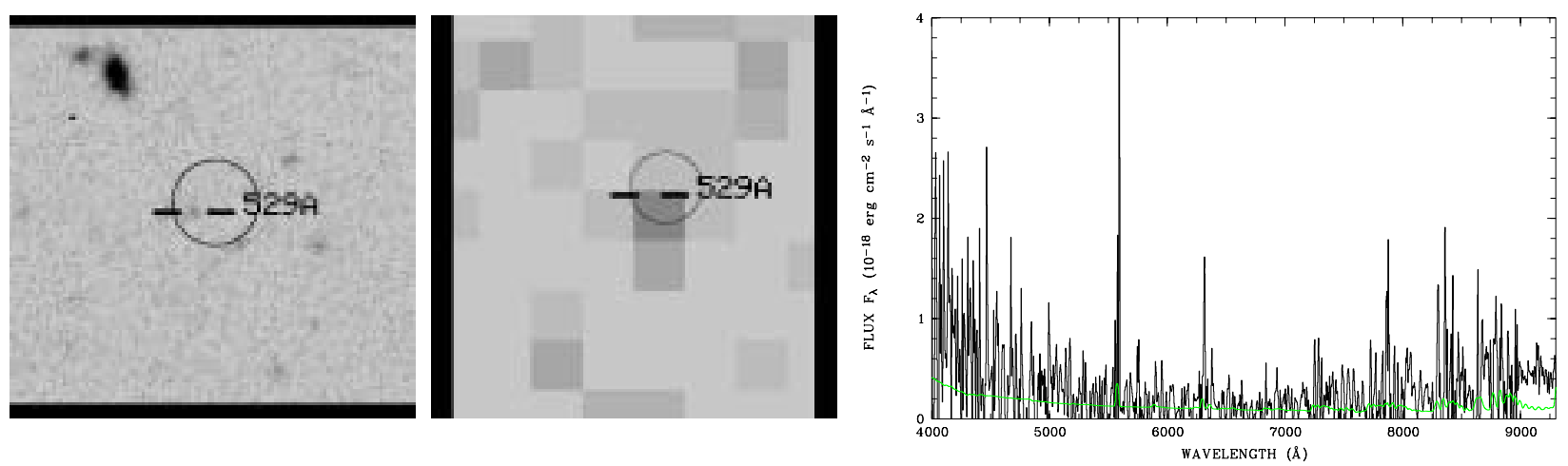

object 529A, no redshift, spectrum too noisy
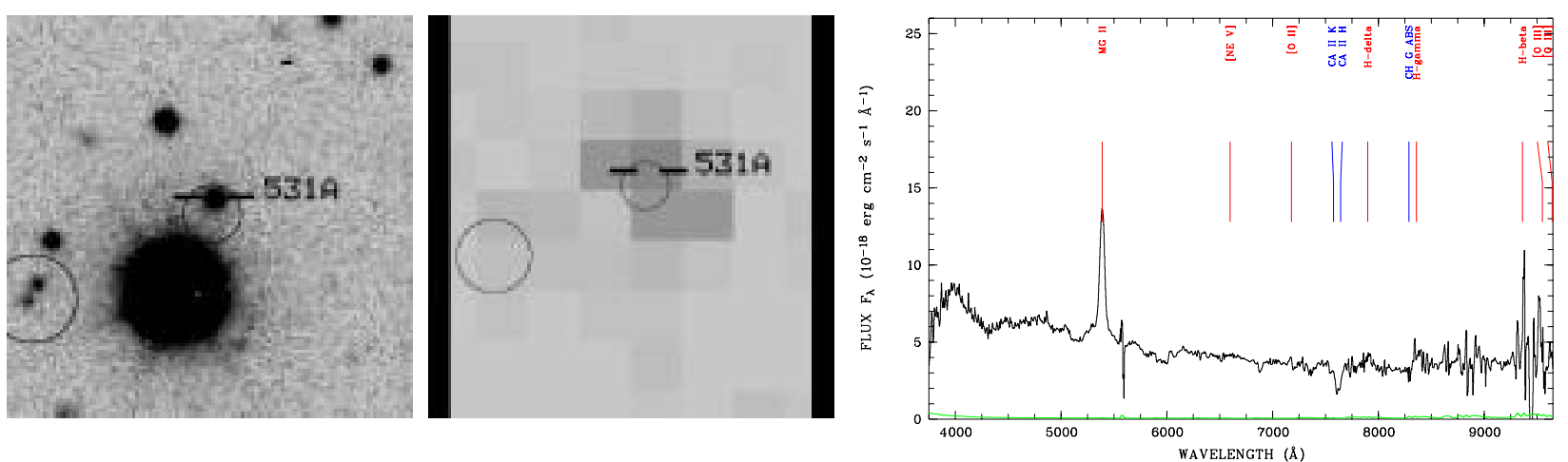

object $531 \mathrm{~A}, \mathrm{z}=0.926$ 

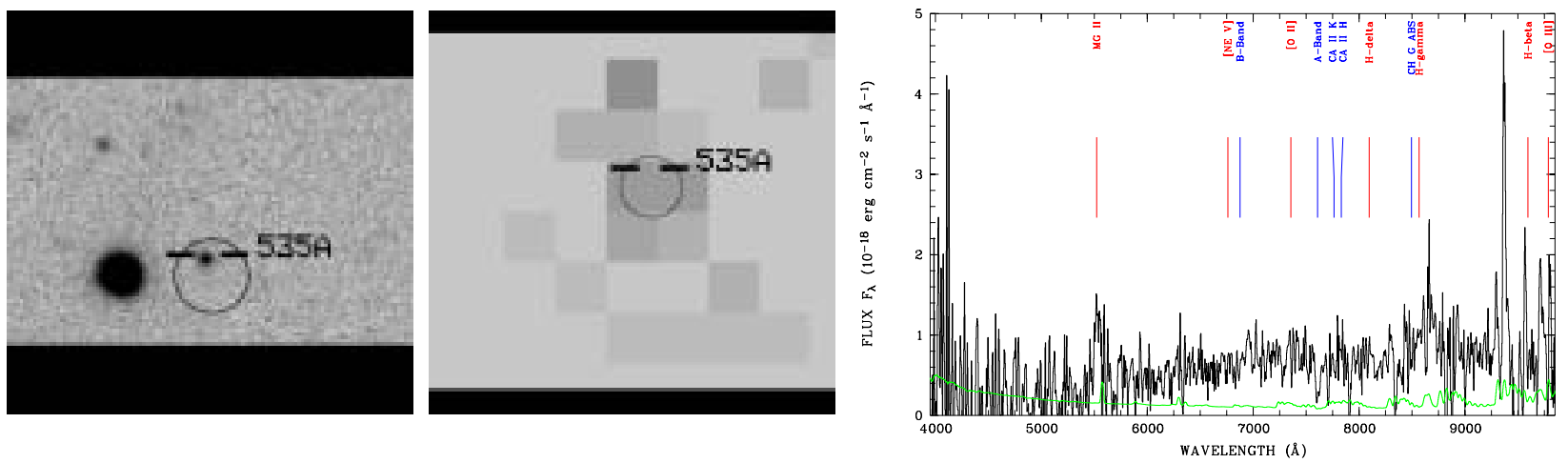

object 535A, z =0.974:@MgII
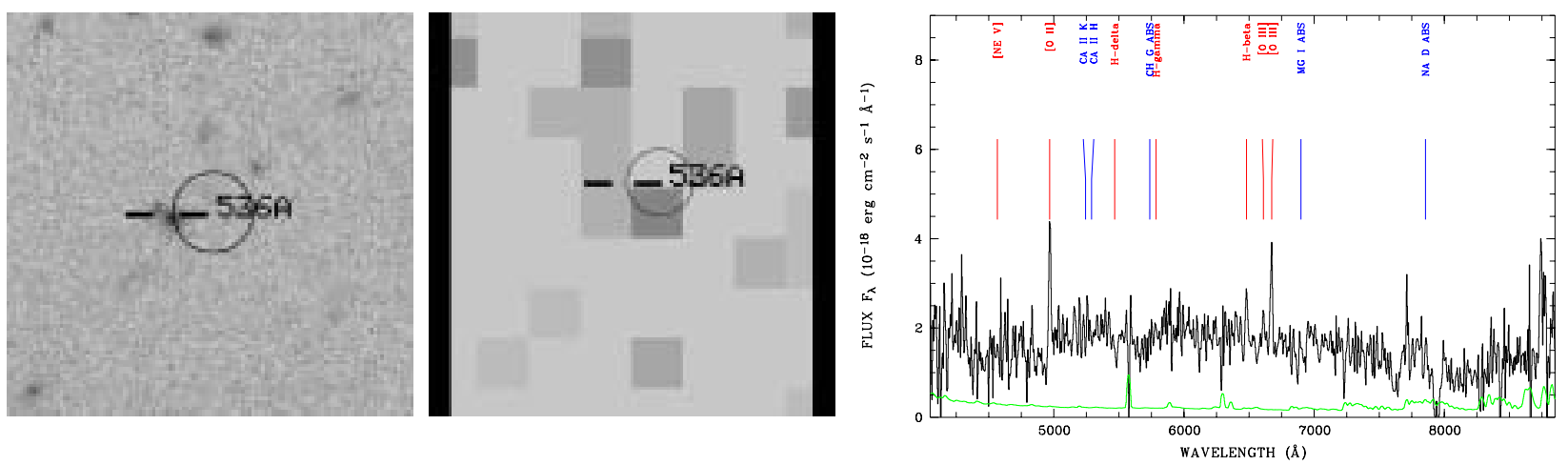

object 536A, z $=0.333$
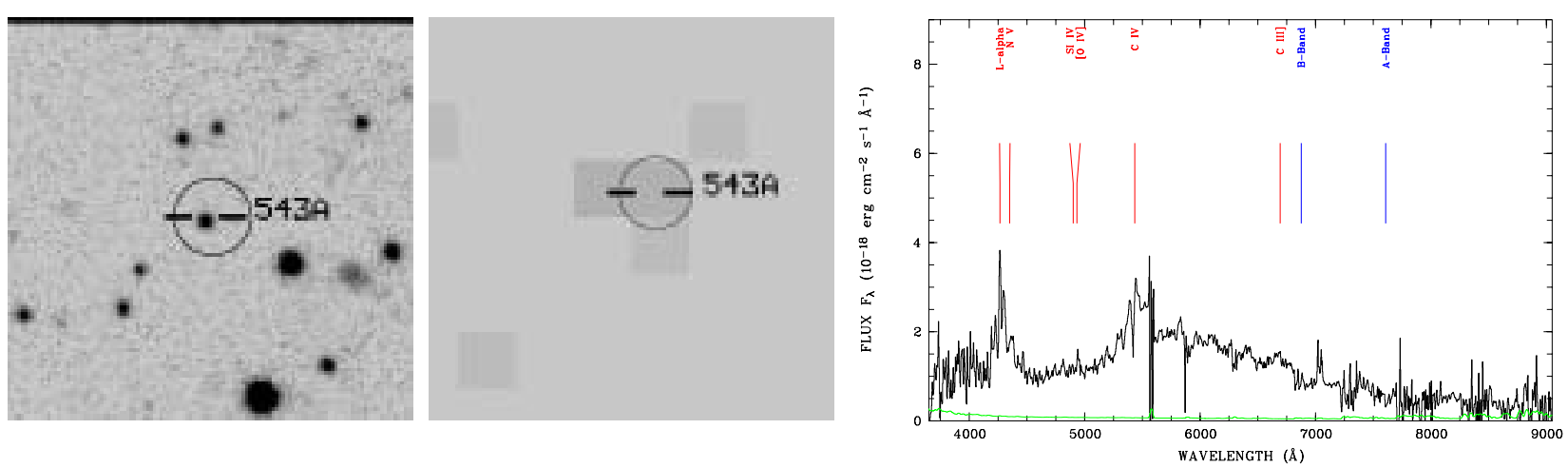

object $543 \mathrm{~A}, \mathrm{z}=2.511$
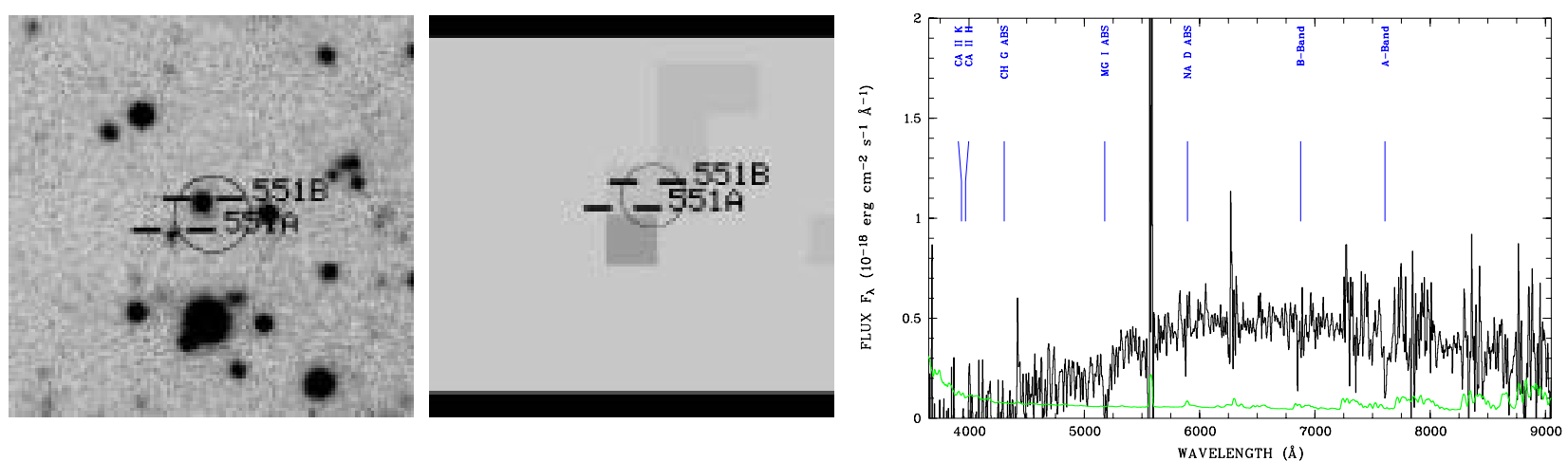

object $551 \mathrm{~A}, \mathrm{z}=0.000$ 
M. Krumpe et al.: The XMM-Newton survey in the Marano field. I., Online Material p 59
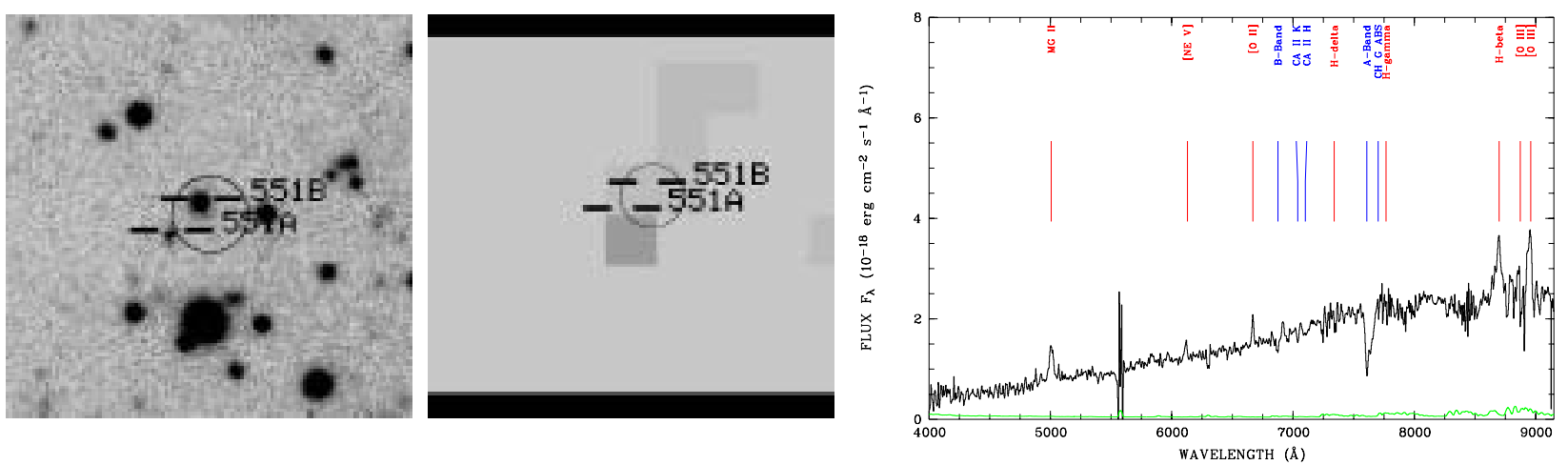

object $551 \mathrm{~B}, \mathrm{z}=0.789$
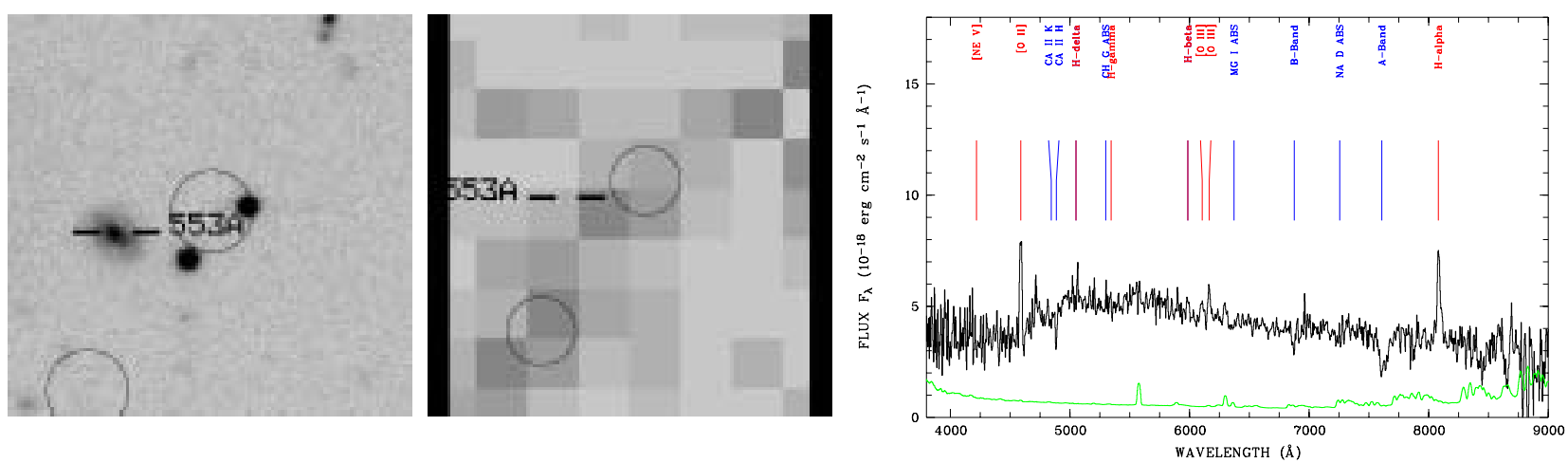

object 553A, $\mathrm{z}=0.231$
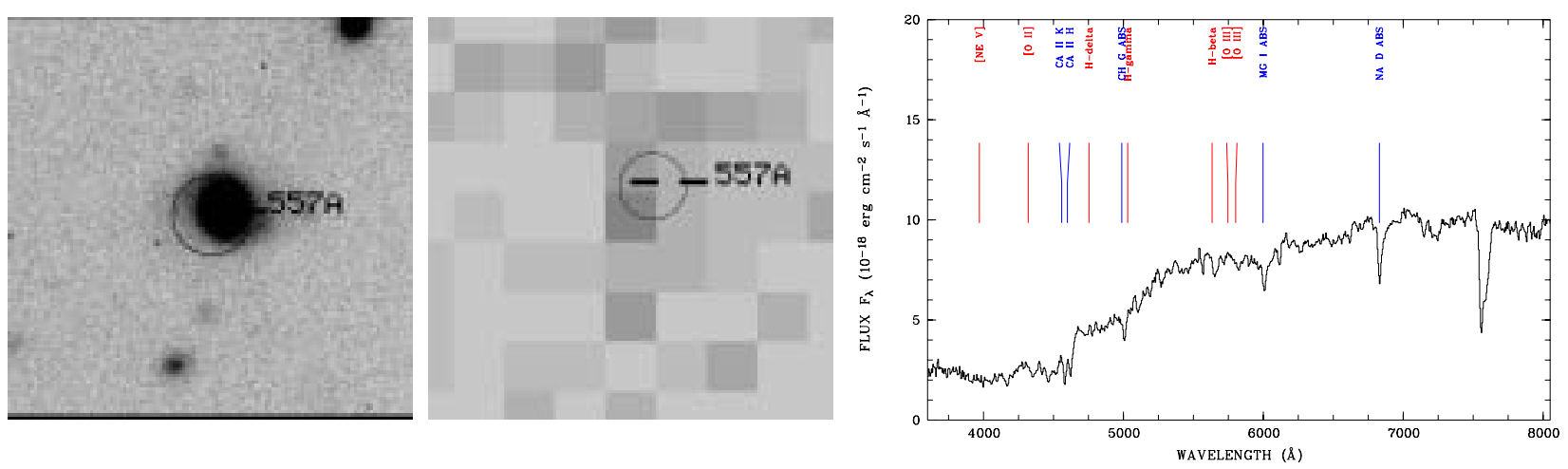

object 557A, $\mathrm{z}=0.16$
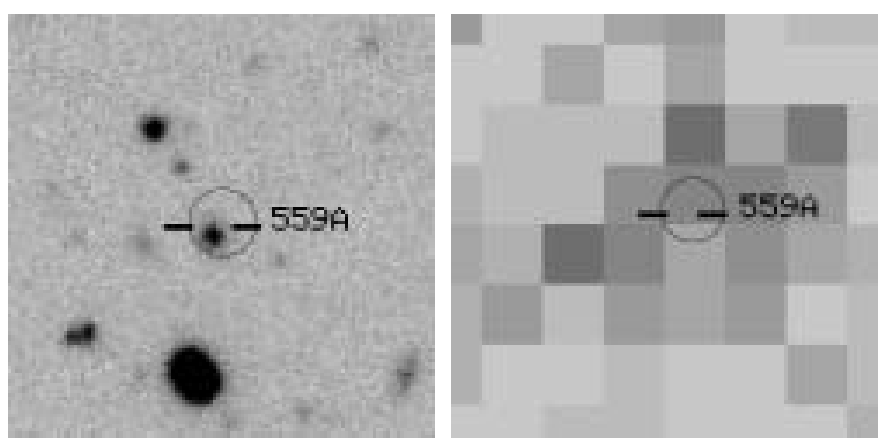

object 559A, $\mathrm{z}=1.180$ 
M. Krumpe et al.: The XMM-Newton survey in the Marano field. I., Online Material p 60
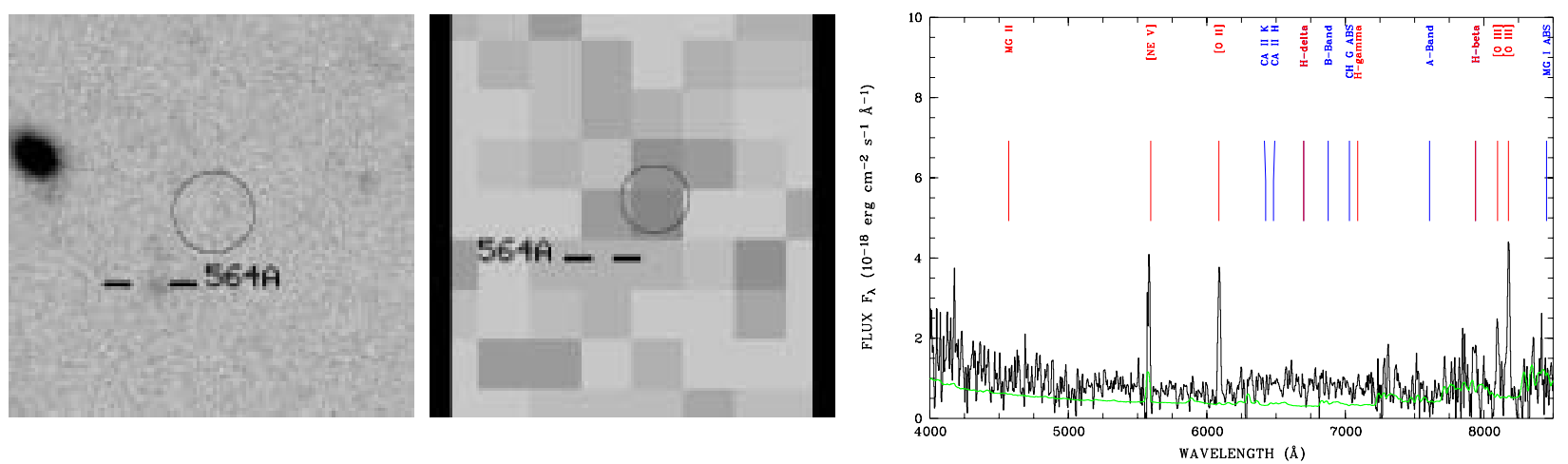

object $564 \mathrm{~A}, \mathrm{z}=0.633$
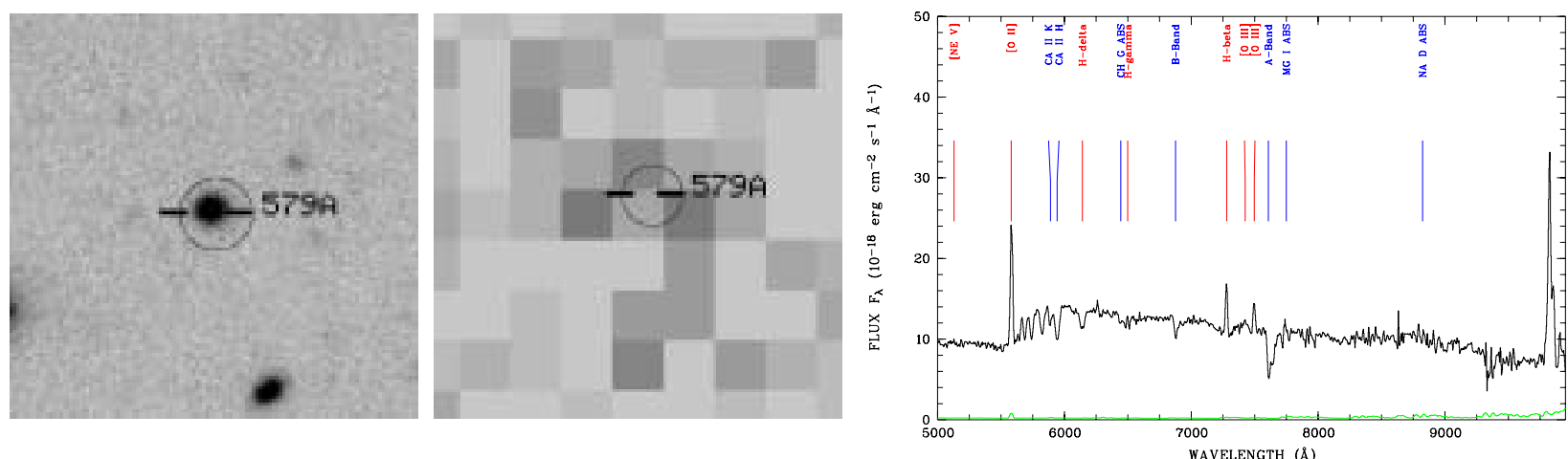

object 579A, z $=0.497$
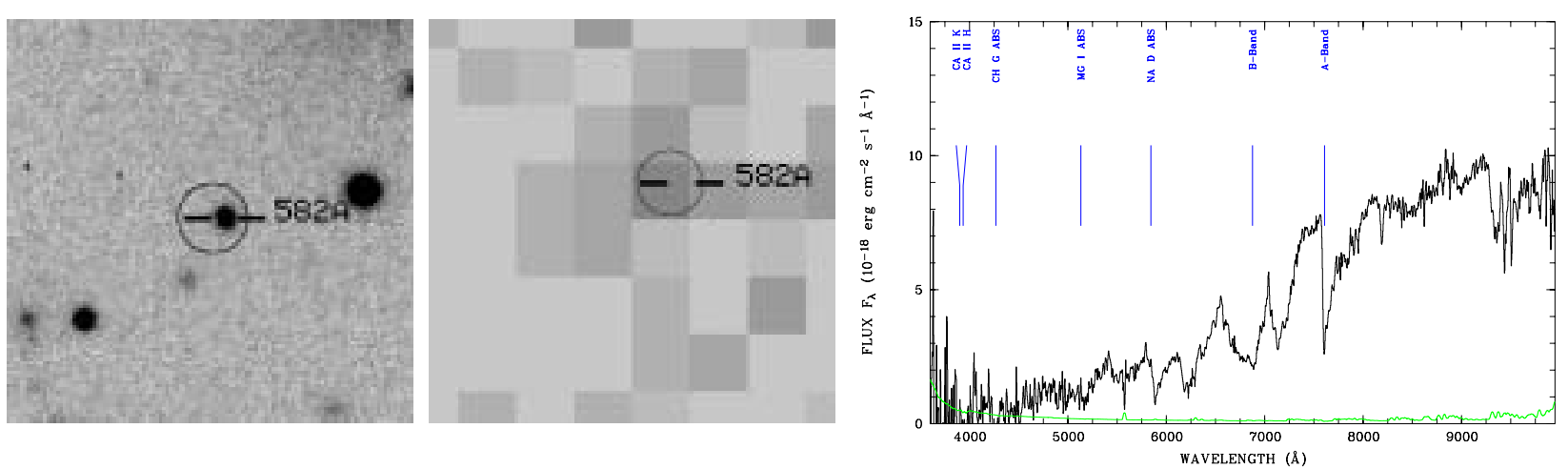

object $582 \mathrm{~A}, \mathrm{z}=0.000$
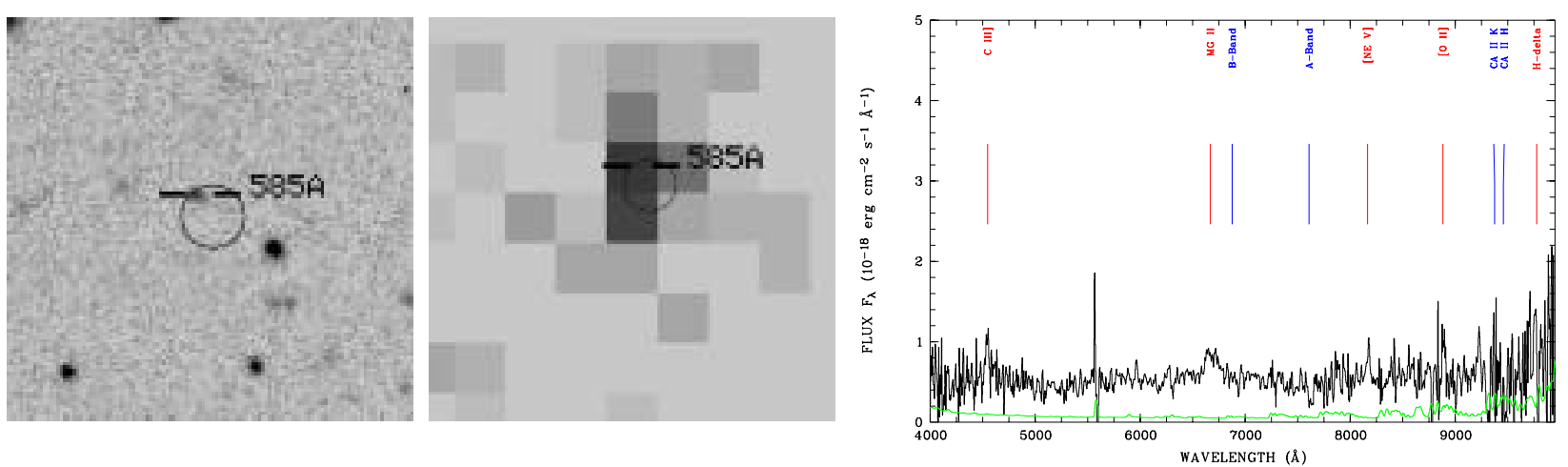

object $585 \mathrm{~A}, \mathrm{z}=1.383$ 
M. Krumpe et al.: The XMM-Newton survey in the Marano field. I., Online Material $p 61$
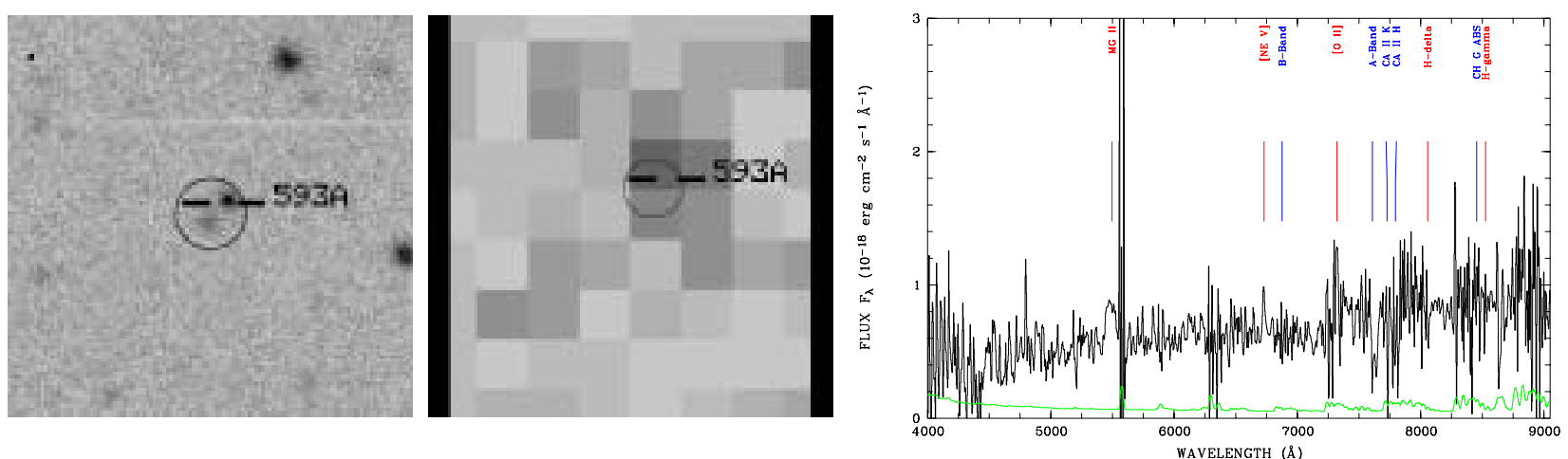

object $593 \mathrm{~A}, \mathrm{z}=0.964$
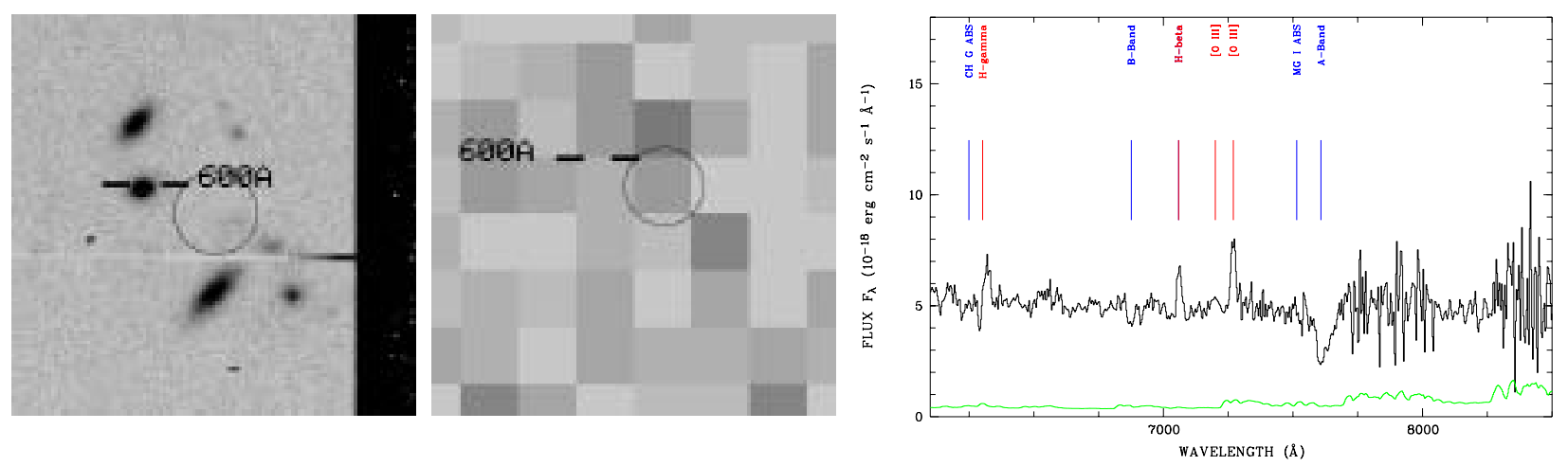

object $600 \mathrm{~A}, \mathrm{z}=0.452$
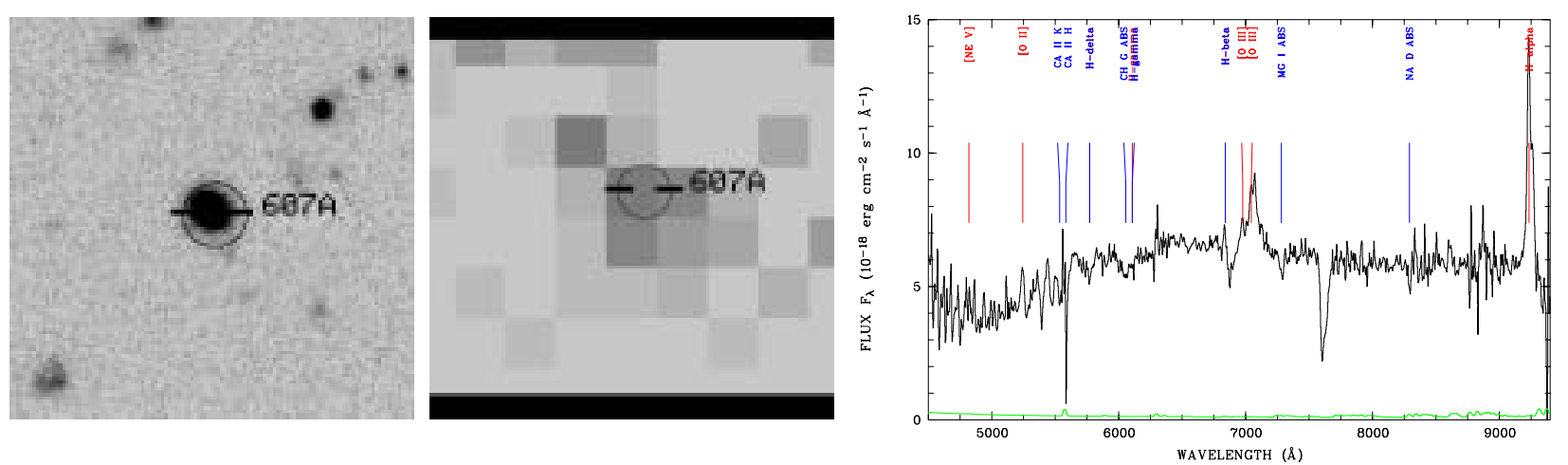

object $607 \mathrm{~A}, \mathrm{z}=0.407$
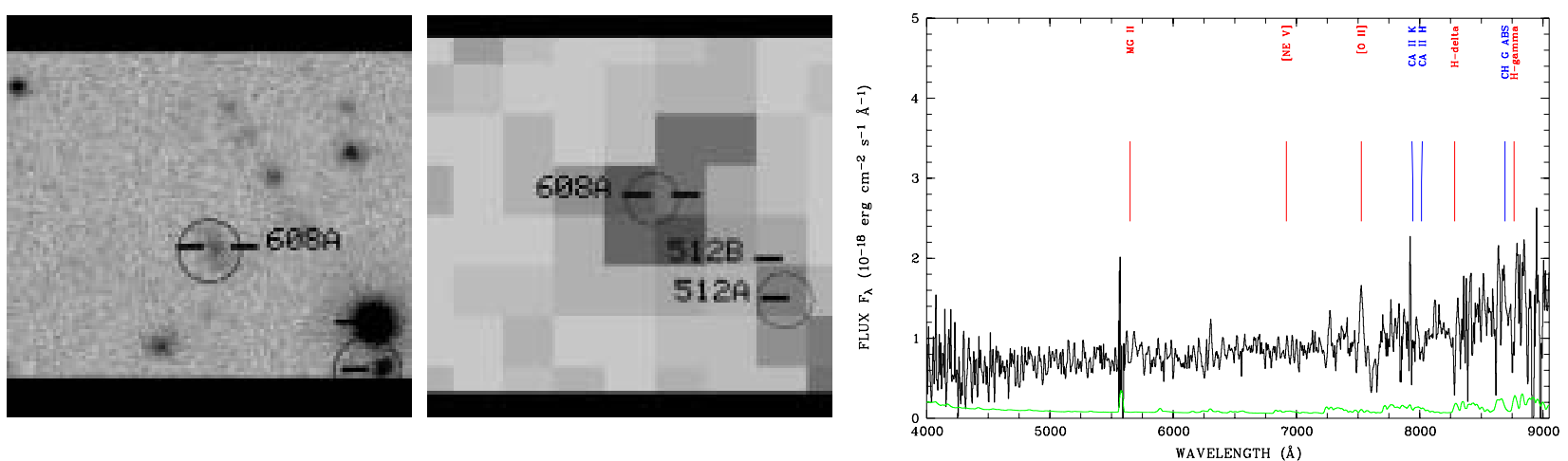

object 608A, z=1.019:@OII \& Ca-Break 
M. Krumpe et al.: The XMM-Newton survey in the Marano field. I., Online Material p 62
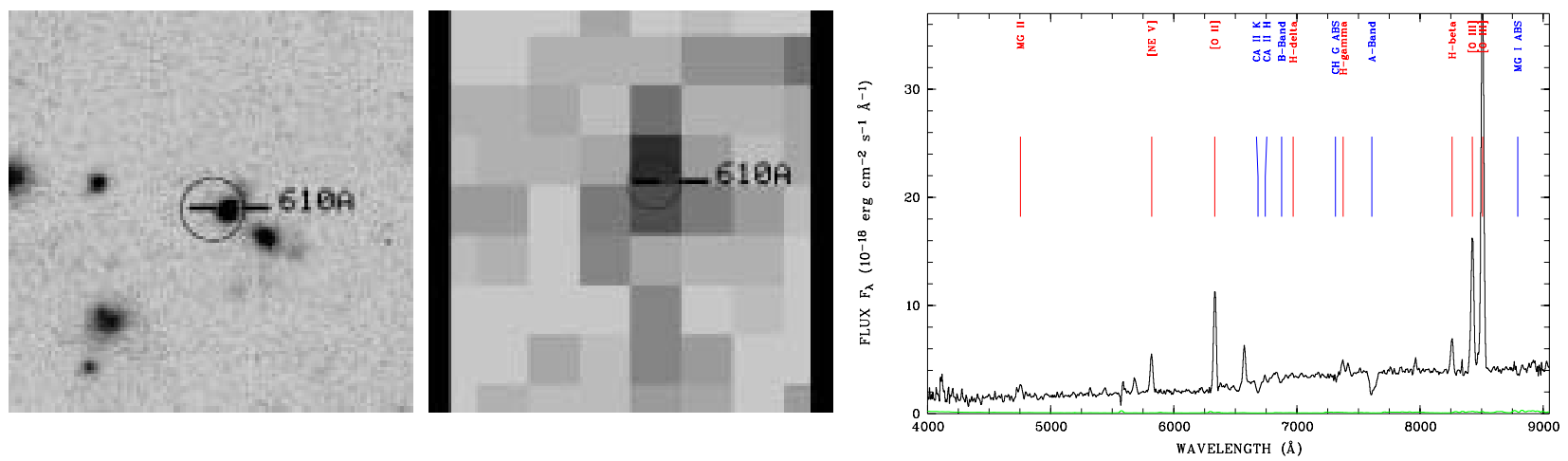

object $610 \mathrm{~A}, \mathrm{z}=0.699$
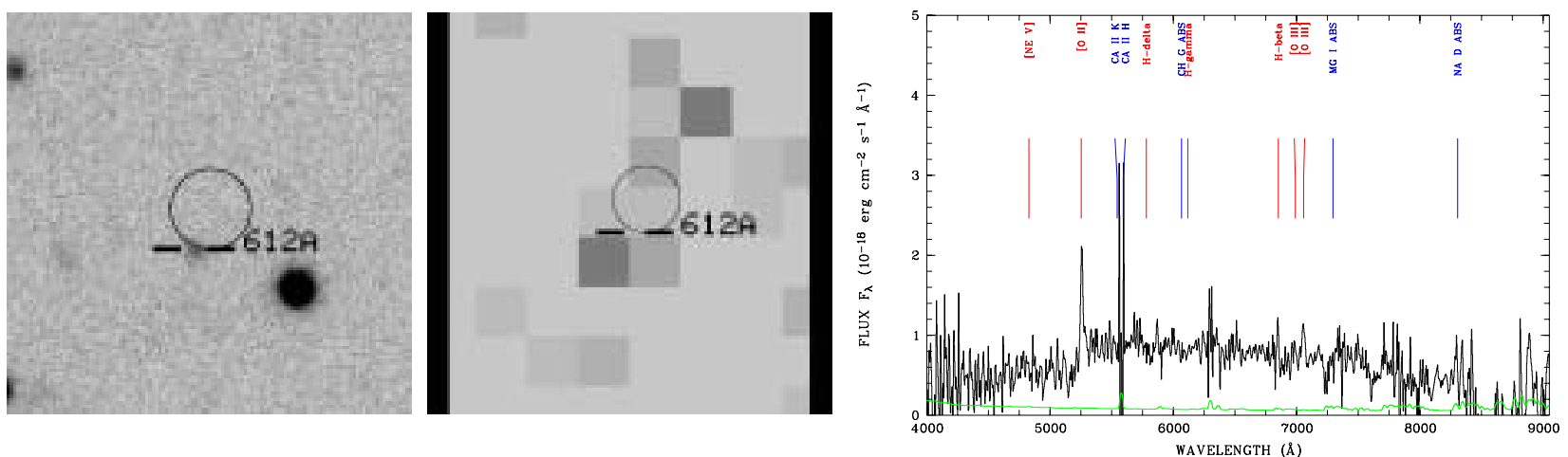

object $612 \mathrm{~A}, \mathrm{z}=0.409$
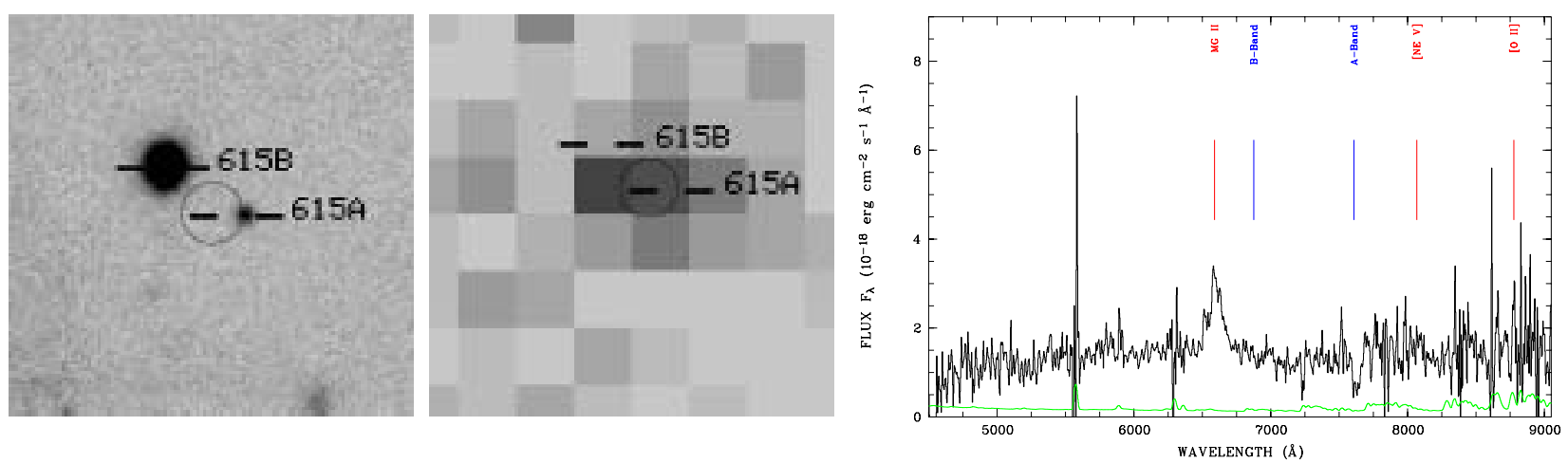

object 615A, z=1.355:@MgII
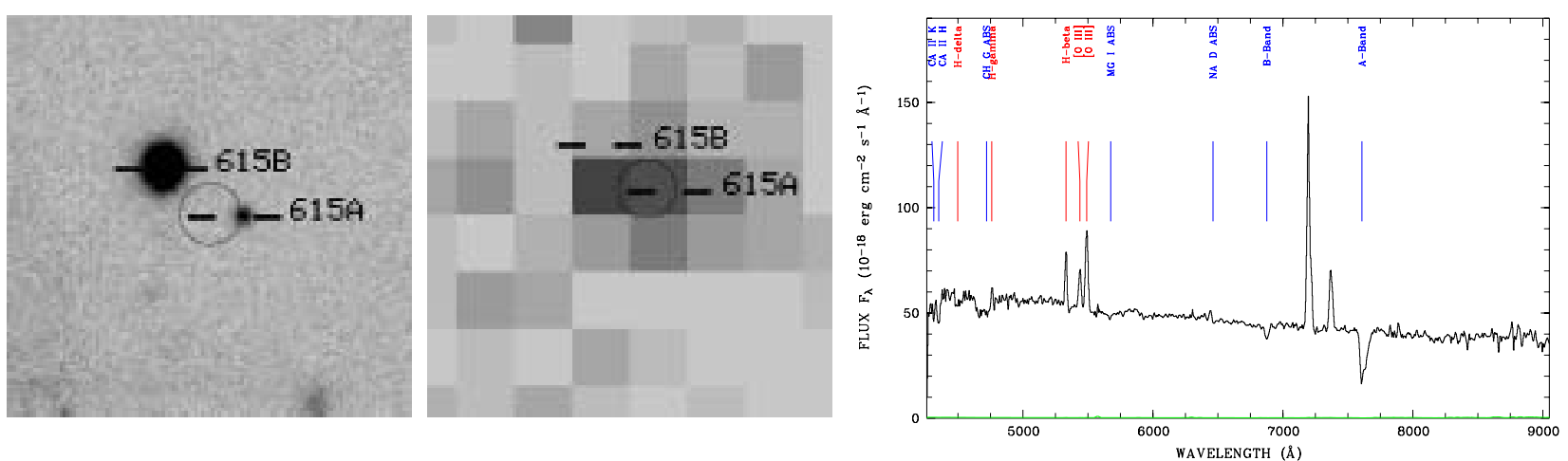

object $615 \mathrm{~B}, \mathrm{z}=0.096$ 
M. Krumpe et al.: The XMM-Newton survey in the Marano field. I., Online Material p 63
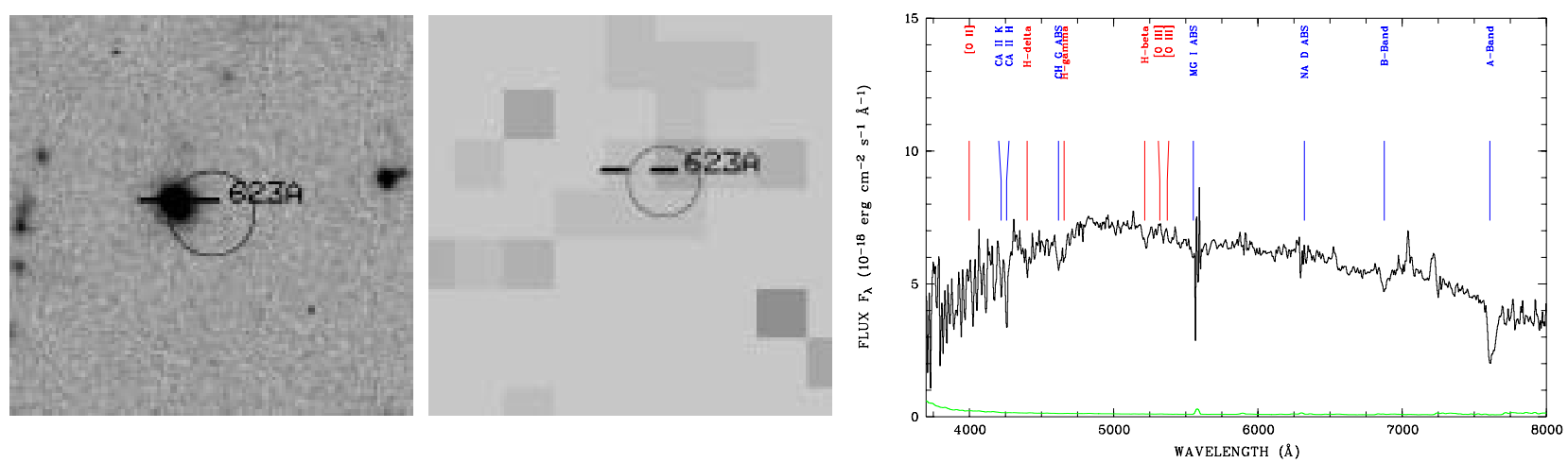

object $623 \mathrm{~A}, \mathrm{z}=0.073$
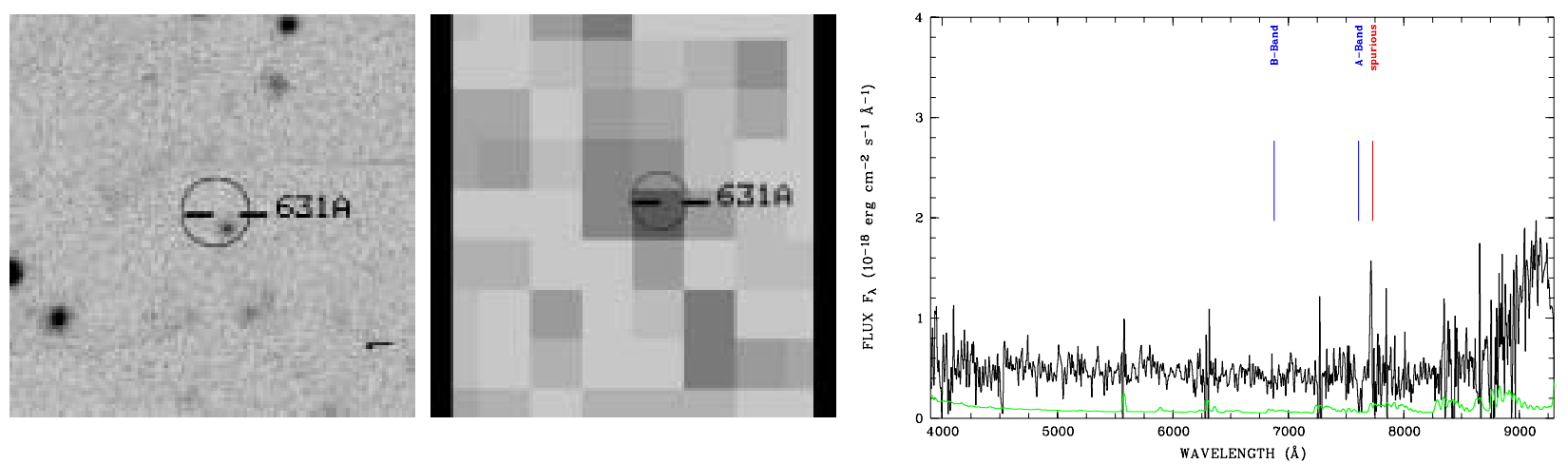

object 631A, no redshift, spectrum too noisy
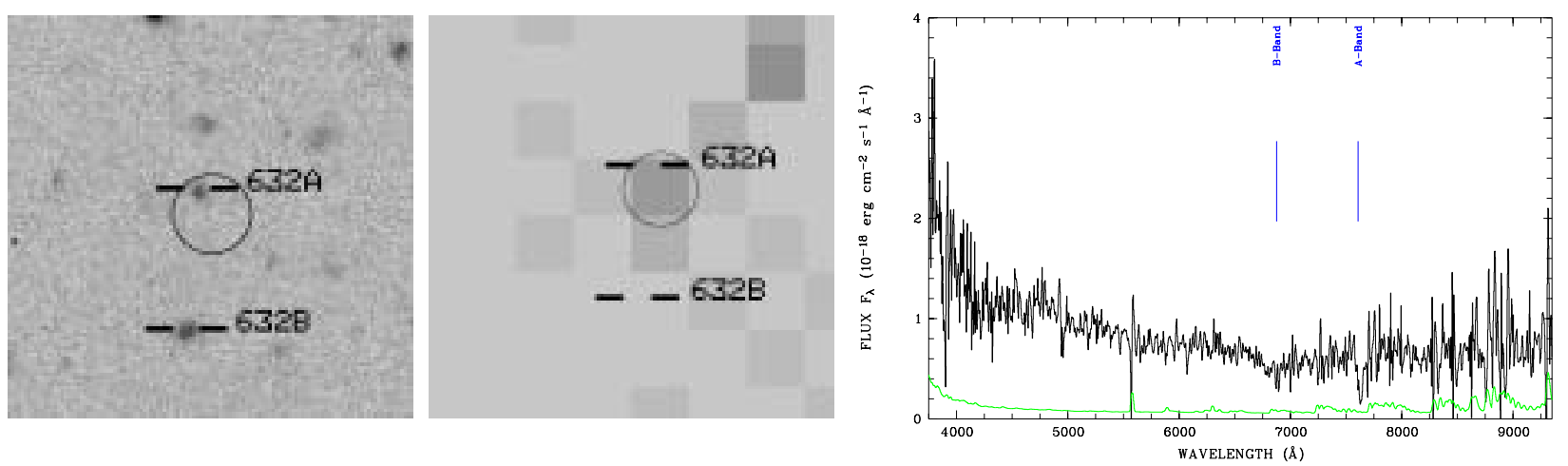

object $632 \mathrm{~A}$, no redshift
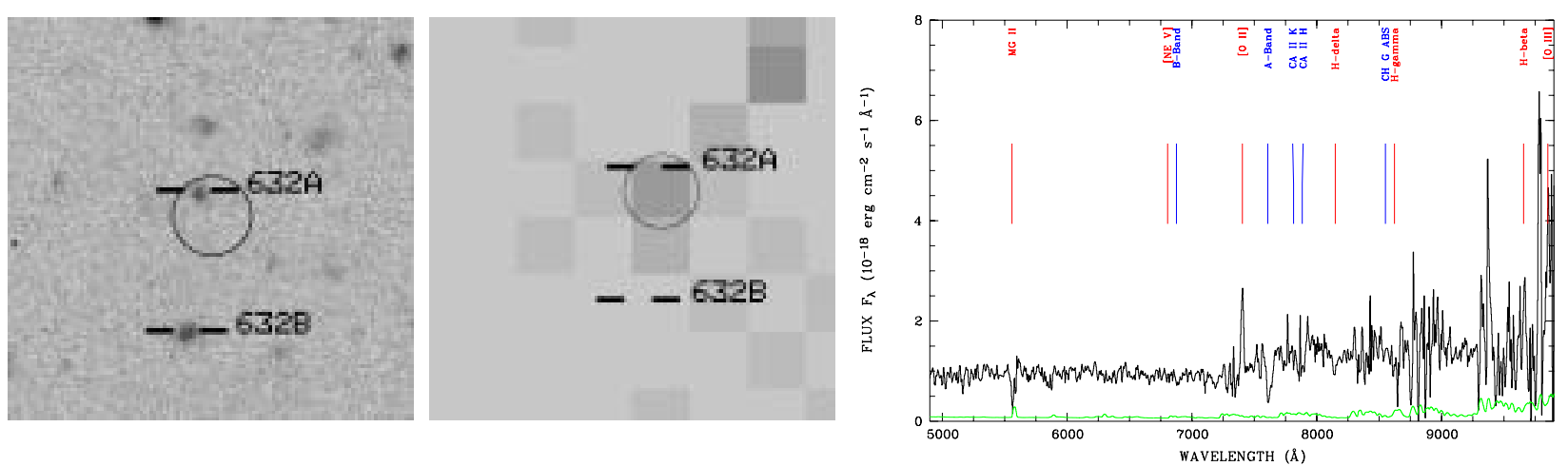

object $632 \mathrm{~B}, \mathrm{z}=0.987$ 

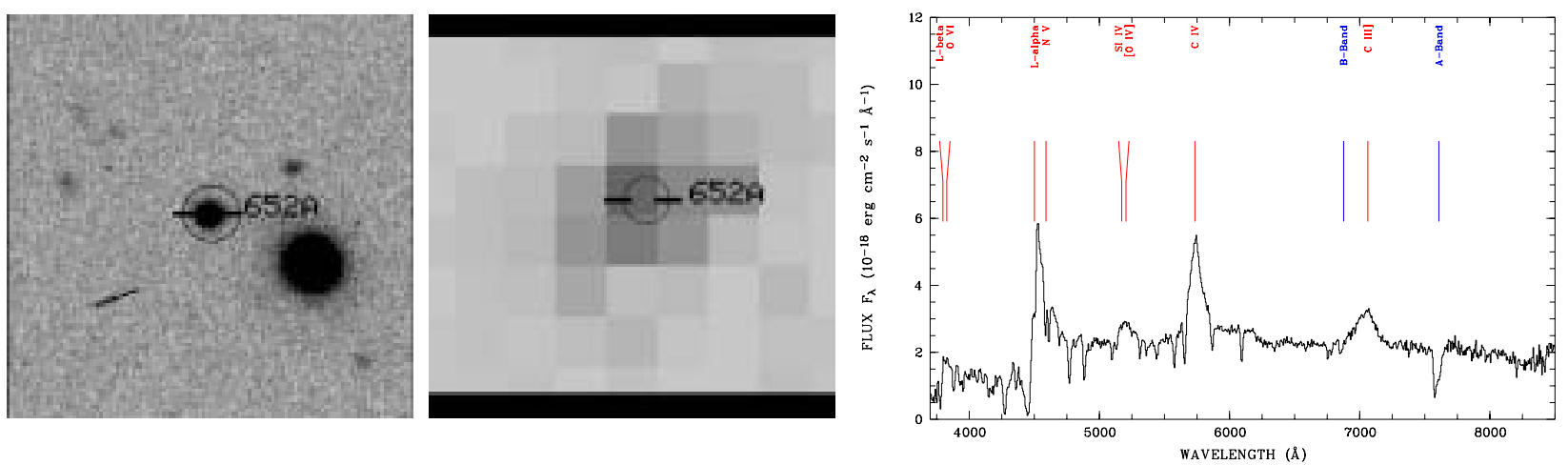

object $652 \mathrm{~A}, \mathrm{z}=2.710$
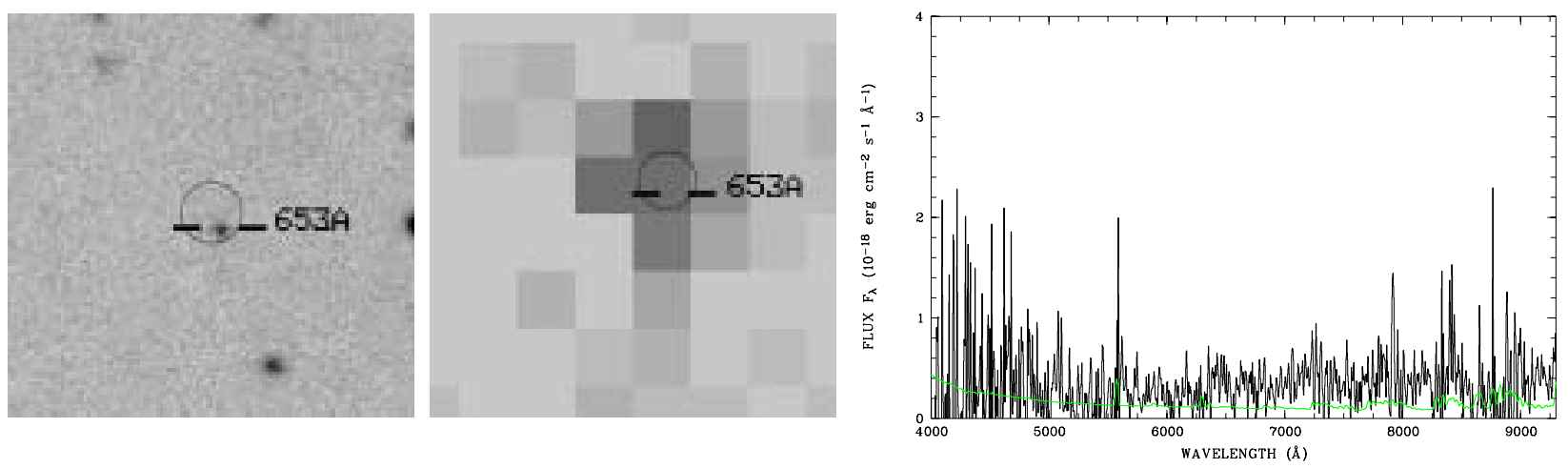

object 653A, no redshift, spectrum too noisy
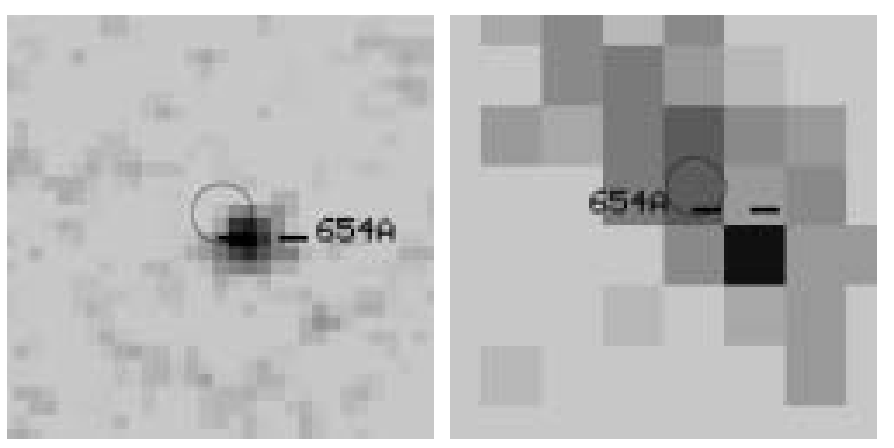

object $654 \mathrm{~A}, \mathrm{z}=2.105$
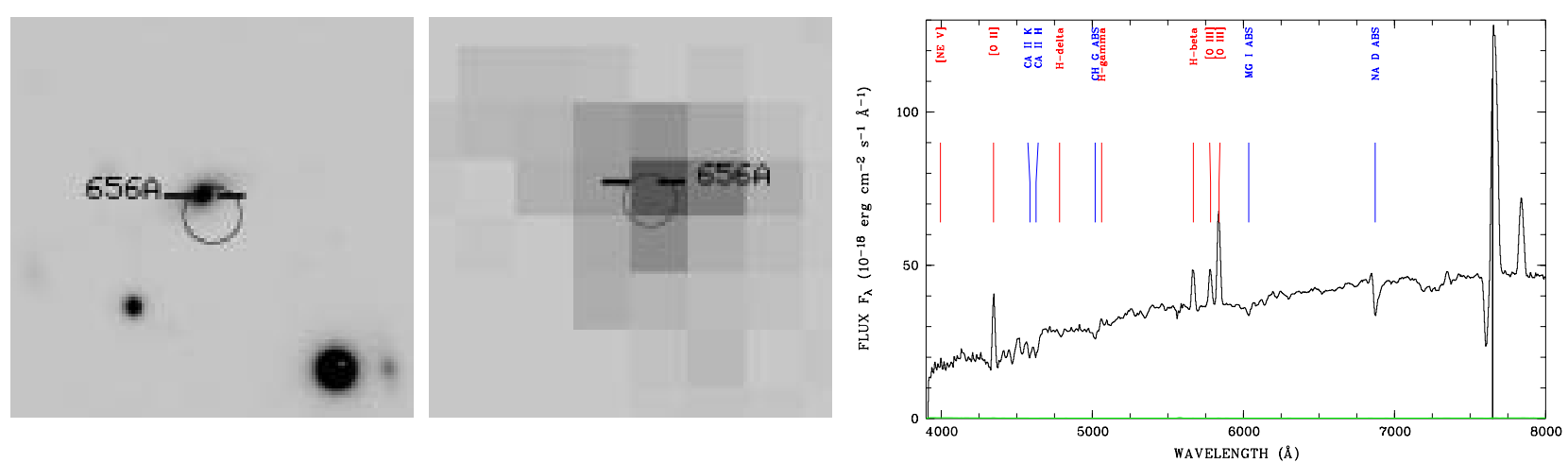

object $656 \mathrm{~A}, \mathrm{z}=0.166$ 
M. Krumpe et al.: The XMM-Newton survey in the Marano field. I., Online Material p 65
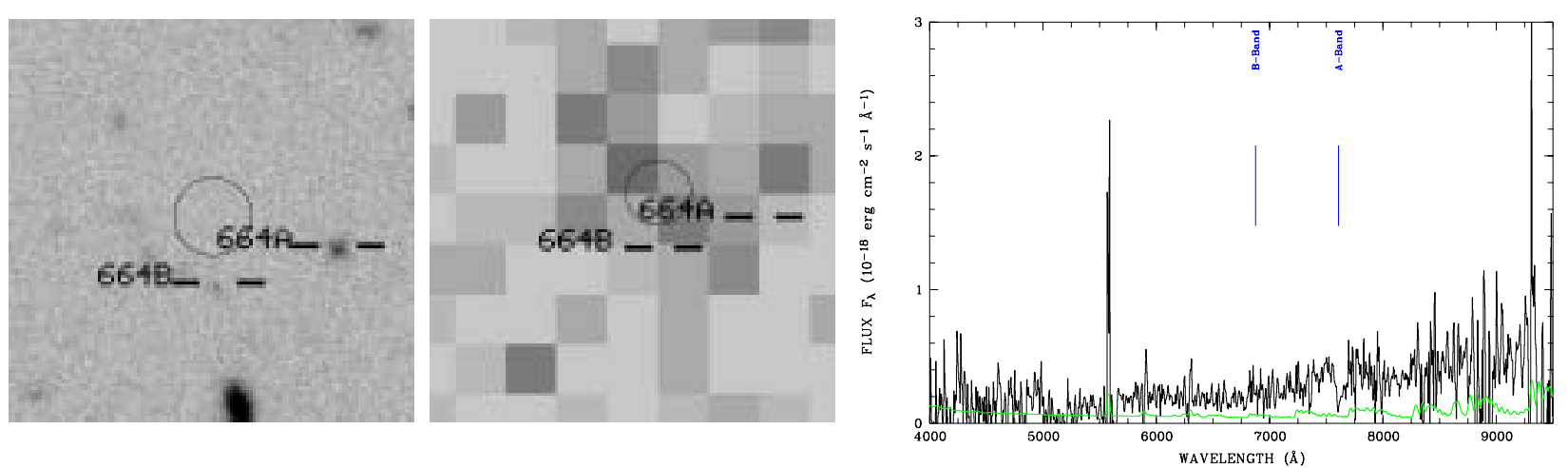

object 664A, no redshift, spectrum too noisy
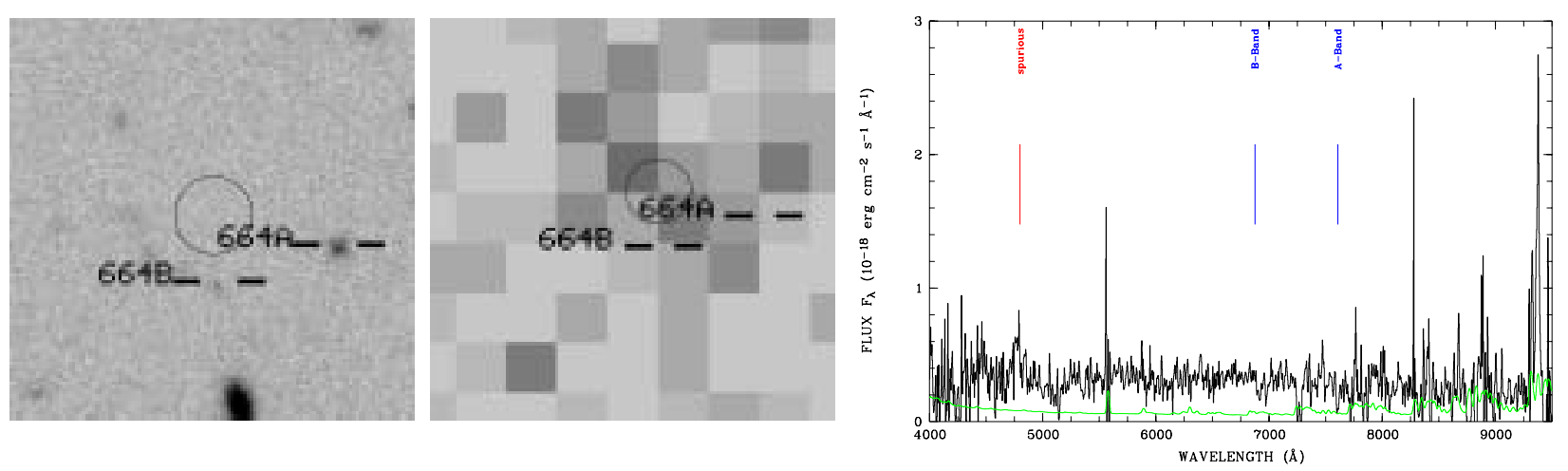

object 664B, no redshift, spectrum too noisy
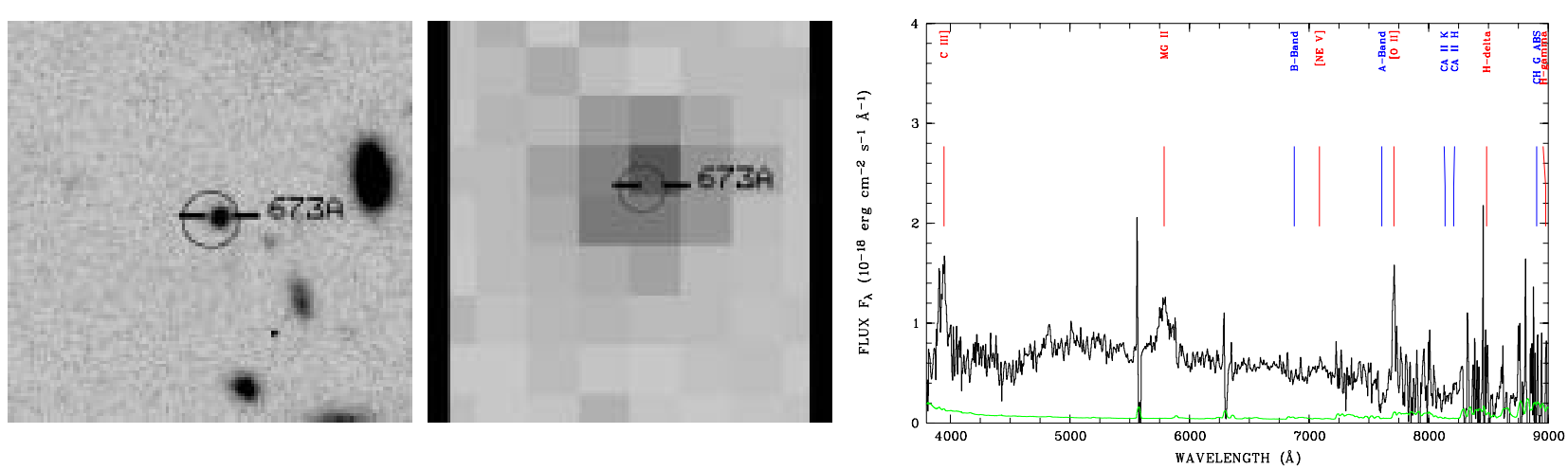

object $673 \mathrm{~A}, \mathrm{z}=1.062$
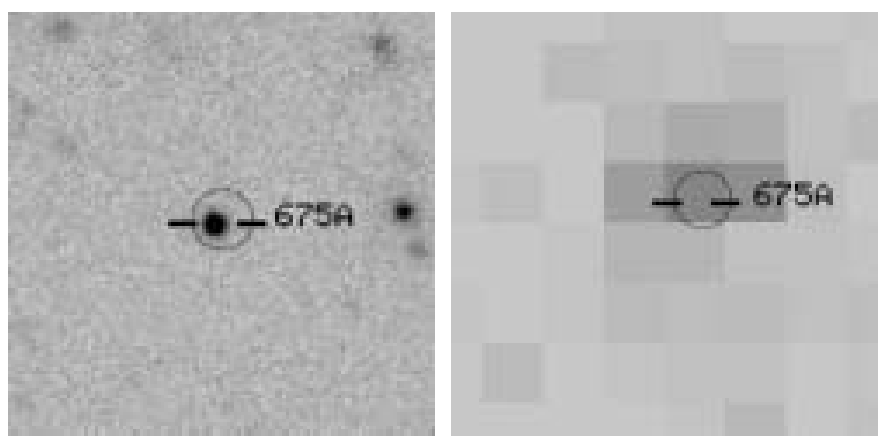

object $675 \mathrm{~A}, \mathrm{z}=1.140$ 

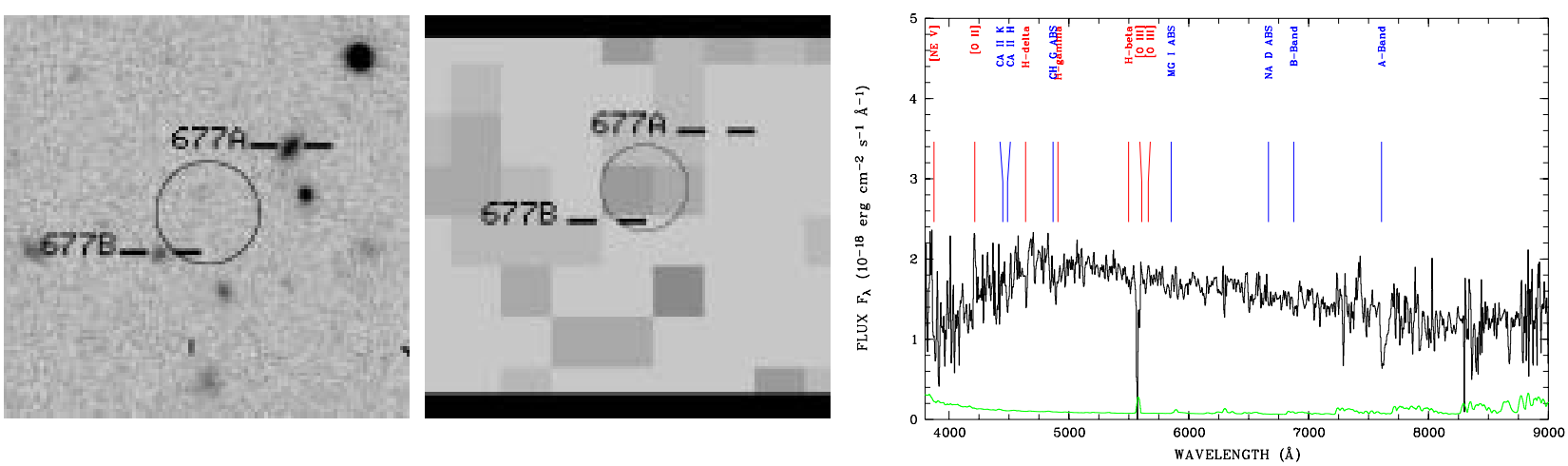

object $677 \mathrm{~A}, \mathrm{z}=0.131$
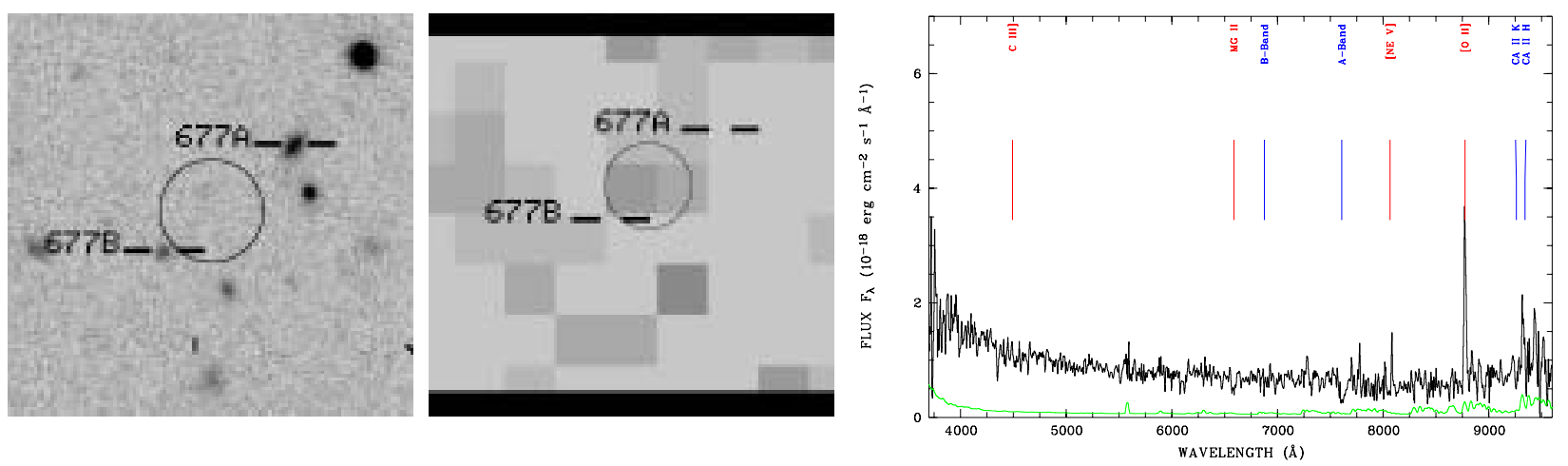

object 677B, z=1.354:@OII
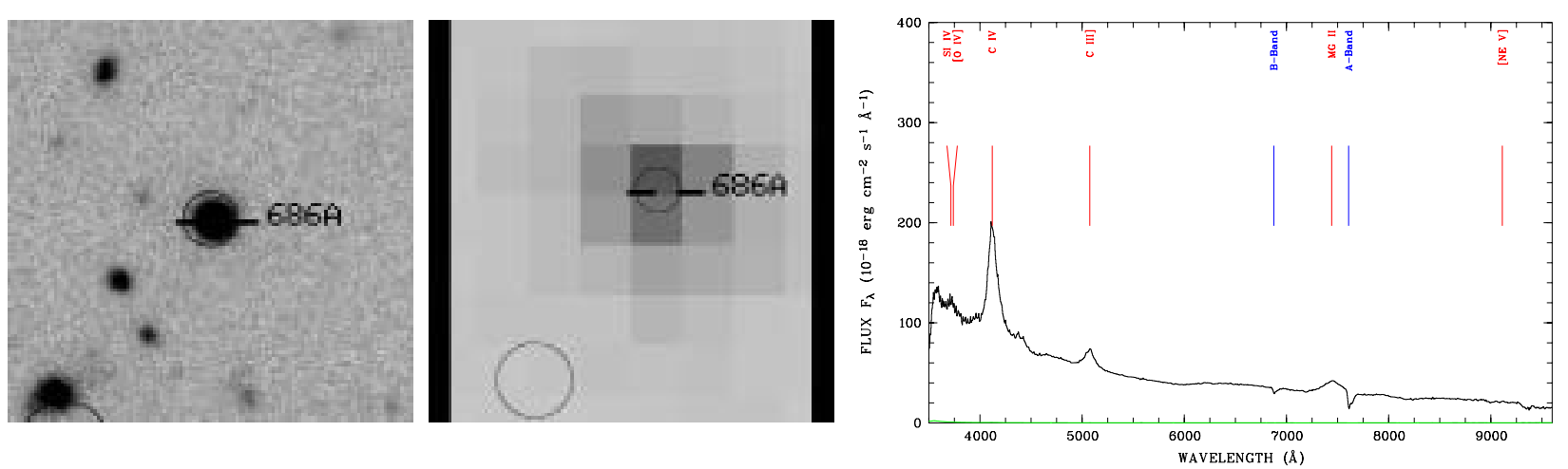

object $686 \mathrm{~A}, \mathrm{z}=1.663$
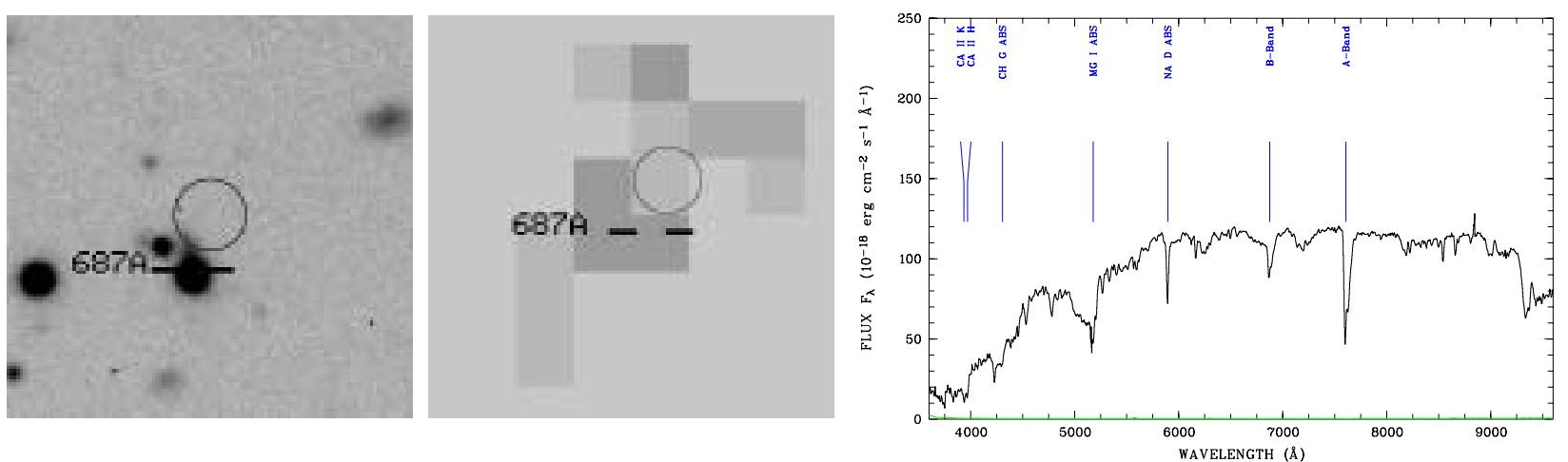

object $687 \mathrm{~A}, \mathrm{z}=0.000$ 
M. Krumpe et al.: The XMM-Newton survey in the Marano field. I., Online Material p 67

\section{Appendix D: Optical table of additional objects}

(1) No

Continuously arbitrary identification number of additional spectroscopically classified sources in the Marano Field. These sources are labelled with the suffix " $M A$ ".

(2) $R A$ [hh:min:s] and (3) Dec [degmin:s]

Right ascension and declination of the spectroscopically classified object.

(4) $z$

Spectroscopic redshift of the classified object.

(5) $K$

SOFI $K$-band magnitude of the spectroscopically classified object, whenever possible.

(6) $R$

WFI $R$-band magnitude of the spectroscopically classified object, whenever possible.

\section{(7) Class}

Classification of the optical identified object. S - star, G - normal galaxy (no emission lines), and $\mathrm{N}$ - narrow emission line galaxy (unresolved emission lines).

Table D.1. Optical data of additional objects.

\begin{tabular}{|c|c|c|c|c|c|c|}
\hline (1) & (2) & (3) & (4) & (5) & (6) & $\overline{\overline{(7)}}$ \\
\hline No & RA & Dec & $z$ & $K$ & $R$ & Class \\
\hline $1 \mathrm{MA}$ & 31608.4 & -552306 & 0.486 & 17.51 & 20.41 & $\bar{N}$ \\
\hline $2 \mathrm{MA}$ & 31619.0 & -551734 & 1.027 & 18.77 & 22.52 & G \\
\hline $3 \mathrm{MA}$ & 31621.8 & -551742 & 0.000 & - & 22.64 & $\mathrm{~S}$ \\
\hline $4 \mathrm{MA}$ & 31626.8 & -551830 & 1.199 & 20.23 & 22.12 & $\mathrm{~N}$ \\
\hline $5 \mathrm{MA}$ & 31635.0 & -551840 & 1.374 & - & 23.89 & $\mathrm{~N}$ \\
\hline $6 \mathrm{MA}$ & 31639.6 & -551850 & 0.491 & 19.02 & 21.84 & $\mathrm{~N}$ \\
\hline 7MA & 31626.1 & -550814 & 0.158 & 19.52 & 20.14 & $\mathrm{~N}$ \\
\hline $8 \mathrm{MA}$ & 31633.8 & -551250 & 0.486 & 20.08 & 22.01 & $\mathrm{~N}$ \\
\hline 9MA & 31607.9 & -550507 & 0.786 & 18.70 & 21.36 & $\mathrm{~N}$ \\
\hline 10MA & 31627.2 & -550423 & 0.235 & 18.56 & 18.47 & $\mathrm{~N}$ \\
\hline $11 \mathrm{MA}$ & 31616.2 & -550238 & 0.420 & - & 21.28 & $\mathrm{~N}$ \\
\hline $12 \mathrm{MA}$ & 31623.8 & -550856 & 0.000 & 17.57 & 22.10 & $\mathrm{~S}$ \\
\hline $14 \mathrm{MA}$ & 31626.5 & -550735 & 1.070 & 18.83 & 21.94 & $\mathrm{~N}$ \\
\hline $15 \mathrm{MA}$ & 31624.6 & -550725 & 0.972 & 17.99 & 21.02 & $\mathrm{~N}$ \\
\hline $16 \mathrm{MA}$ & 31331.0 & -551356 & 0.000 & - & 21.87 & S \\
\hline $17 \mathrm{MA}$ & 31336.7 & -551446 & 0.579 & 18.09 & 21.96 & B \\
\hline $18 \mathrm{MA}$ & 31342.3 & -551431 & 0.583 & 17.61 & 21.24 & $\mathrm{~N}$ \\
\hline 19MA & 31344.3 & -551434 & 0.583 & 17.68 & 21.30 & $\mathrm{~N}$ \\
\hline $20 \mathrm{MA}$ & 31348.8 & -551410 & 0.000 & 18.95 & 20.07 & $\mathrm{~S}$ \\
\hline $21 \mathrm{MA}$ & 31350.4 & -551320 & 0.552 & 18.26 & 22.40 & $\mathrm{~N}$ \\
\hline $22 \mathrm{MA}$ & 31352.8 & -551337 & 0.000 & 19.82 & 22.29 & S \\
\hline $23 \mathrm{MA}$ & 31306.8 & -551304 & 0.254 & - & 20.09 & G \\
\hline $24 \mathrm{MA}$ & 31311.4 & -551139 & 0.000 & - & 23.10 & $\mathrm{~S}$ \\
\hline $25 \mathrm{MA}$ & 31318.2 & -551304 & 0.865 & - & - & $\mathrm{N}$ \\
\hline $26 \mathrm{MA}$ & 31319.0 & -551137 & 0.000 & - & 20.81 & $\mathrm{~S}$ \\
\hline $27 \mathrm{MA}$ & 31324.5 & -550311 & 0.000 & 18.19 & 22.54 & $\mathrm{~S}$ \\
\hline $28 \mathrm{MA}$ & $\begin{array}{ll}31328.3 \\
\end{array}$ & -550138 & 0.800 & 19.46 & 22.14 & $\mathrm{~N}$ \\
\hline 30MA & 31514.6 & -551033 & 0.000 & - & 22.79 & S \\
\hline $31 \mathrm{MA}$ & 31545.7 & -551249 & 0.628 & 17.12 & 21.31 & G \\
\hline $32 \mathrm{MA}$ & 31543.7 & -550857 & 0.771 & 19.42 & 23.17 & G \\
\hline
\end{tabular}


M. Krumpe et al.: The XMM-Newton survey in the Marano field. I., Online Material p 68

Table D.1. continued.

\begin{tabular}{rrrrrrrr}
\hline \hline (1) & & $(2)$ & $(3)$ & $(4)$ & $(5)$ & $(6)$ & $(7)$ \\
No & RA & Dec & $z$ & $K$ & $R$ & Class \\
\hline 33MA & 31414.4 & -551938 & 0.000 & - & 23.08 & $\mathrm{~S}$ \\
34MA & 31415.2 & -552143 & 0.813 & 18.16 & 22.62 & $\mathrm{~N}$ \\
35MA & 31411.5 & -552118 & 0.869 & 18.36 & 22.43 & $\mathrm{~N}$ \\
36MA & 31408.5 & -552024 & 0.000 & 18.77 & 22.64 & $\mathrm{~S}$ \\
37MA & 31311.5 & -552113 & 0.907 & - & 22.25 & $\mathrm{~N}$ \\
38MA & 31314.2 & -552632 & 0.554 & - & 21.98 & $\mathrm{~N}$ \\
39MA & 31300.8 & -552556 & 0.818 & - & 21.03 & $\mathrm{~N}$ \\
40MA & 31301.8 & -552545 & 0.816 & - & 20.85 & $\mathrm{~N}$ \\
41MA & 31305.5 & -552528 & 0.552 & - & 21.11 & $\mathrm{~N}$ \\
42MA & 31330.3 & -552442 & 0.613 & - & 22.89 & $\mathrm{~N}$ \\
43MA & 31331.1 & -552429 & 0.000 & - & - & $\mathrm{S}$ \\
44MA & 31333.3 & -552415 & 0.526 & - & 21.51 & $\mathrm{~N}$ \\
45MA & 31441.4 & -550434 & 0.704 & - & 22.15 & $\mathrm{~N}$ \\
46MA & 31448.1 & -550304 & 0.702 & 16.86 & 20.67 & $\mathrm{~N}$ \\
47MA & 31452.7 & -550549 & 0.460 & 16.40 & 19.32 & $\mathrm{G}$ \\
48MA & 31452.8 & -552439 & 0.228 & - & 18.27 & $\mathrm{G}$ \\
49MA & 31449.0 & -552424 & 0.159 & - & 17.43 & $\mathrm{G}$ \\
50MA & 31524.2 & -551404 & 0.774 & - & 22.16 & $\mathrm{~N}$ \\
51MA & 31532.4 & -551419 & 0.700 & - & 20.32 & $\mathrm{~N}$ \\
52MA & 31540.1 & -551234 & 0.631 & 19.22 & 21.66 & $\mathrm{~N}$ \\
53MA & 31544.6 & -551418 & 0.275 & 18.94 & 21.27 & $\mathrm{~N}$ \\
54MA & 31546.3 & -551355 & 0.815 & 19.17 & 22.89 & $\mathrm{~N}$ \\
55MA & 31548.6 & -551357 & 0.475 & 19.91 & 22.48 & $\mathrm{~N}$ \\
56MA & 31514.0 & -551710 & 0.813 & - & 20.99 & $\mathrm{~N}$ \\
57MA & 31516.9 & -551707 & 0.000 & - & 22.09 & $\mathrm{~S}$ \\
58MA & 31520.7 & -551417 & 0.500 & - & 19.87 & $\mathrm{~N}$ \\
59MA & 31521.8 & -551411 & 0.495 & - & 22.97 & $\mathrm{G}$ \\
60MA & 31516.9 & -550613 & 0.840 & 18.38 & 21.81 & $\mathrm{~N}$ \\
61MA & 31608.3 & -552317 & 0.071 & 15.23 & 17.50 & $\mathrm{~N}$ \\
62MA & 31503.4 & -551637 & 0.000 & 15.55 & 17.35 & $\mathrm{~S}$ \\
\hline & & & & & &
\end{tabular}

\section{Appendix E: Optical charts and spectra of additional optical classifications}

For explanation see Appendix C. The only difference is the centring to the optical position, since no X-ray detection is quantified.
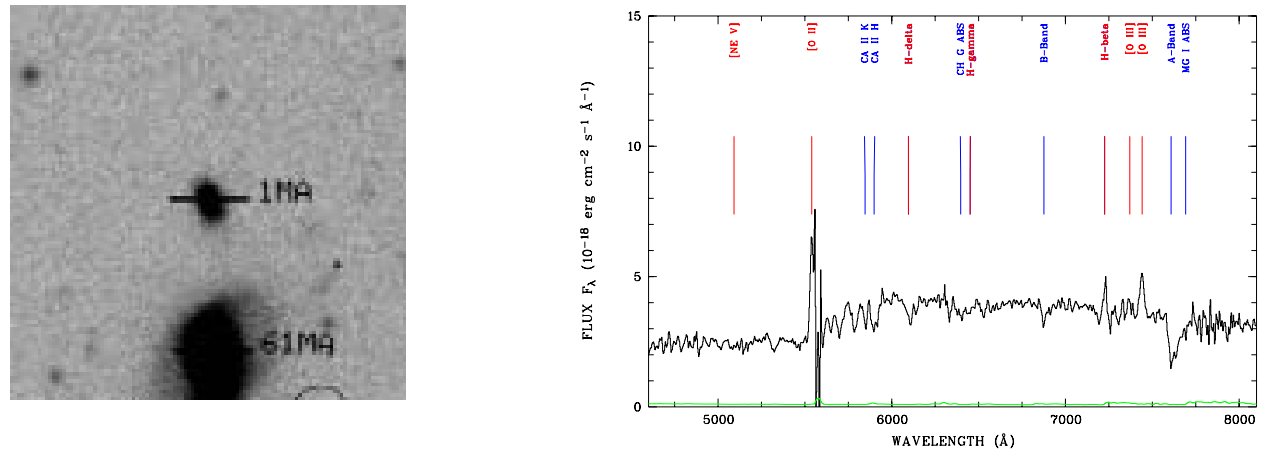

object $1 \mathrm{MA}, \mathrm{z}=0.486$
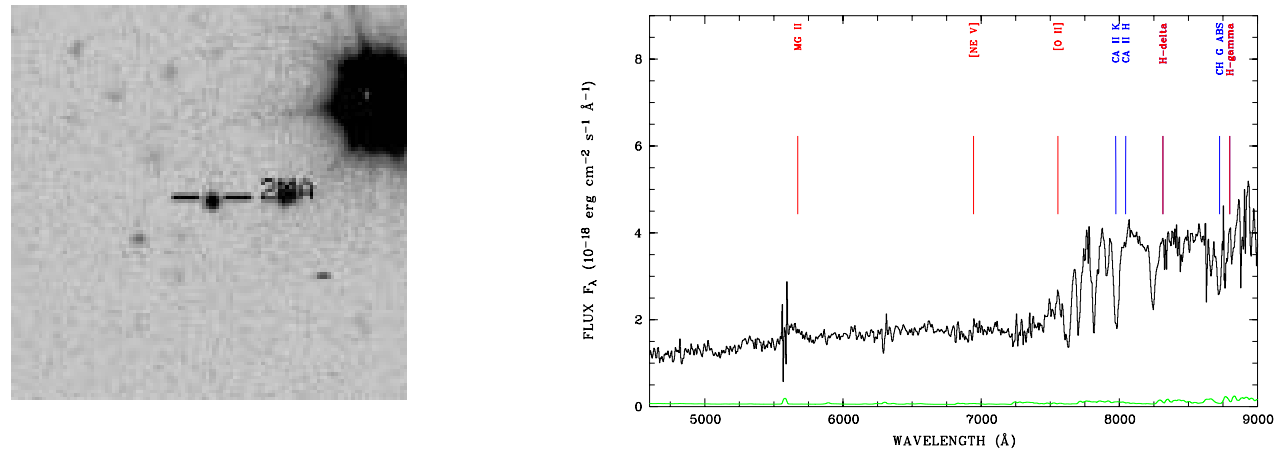

object 2MA, $\mathrm{z}=1.027$ 

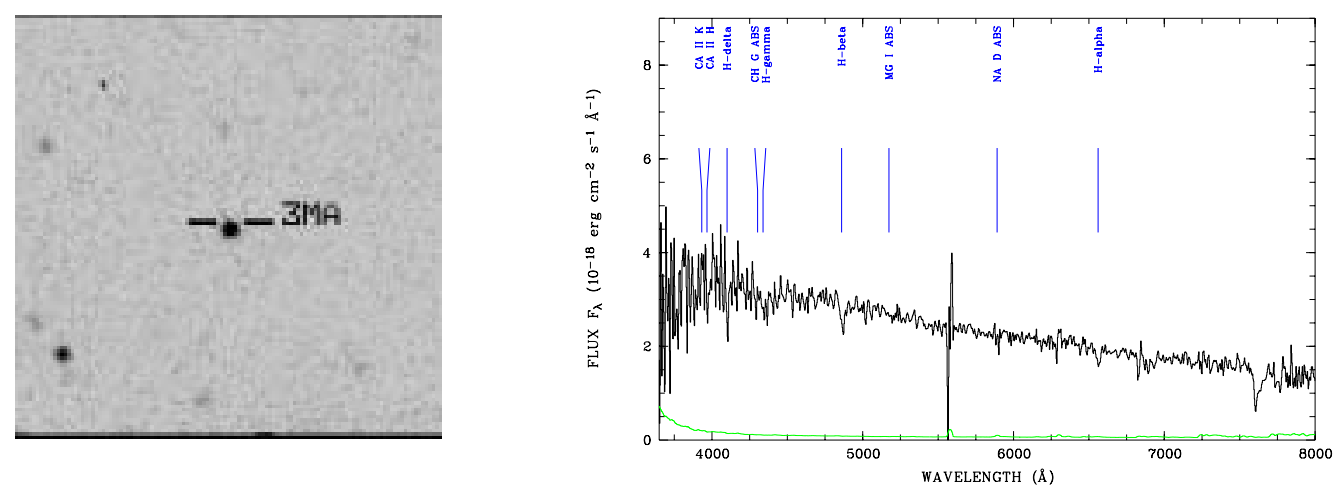

object $3 \mathrm{MA}, \mathrm{z}=0.000$
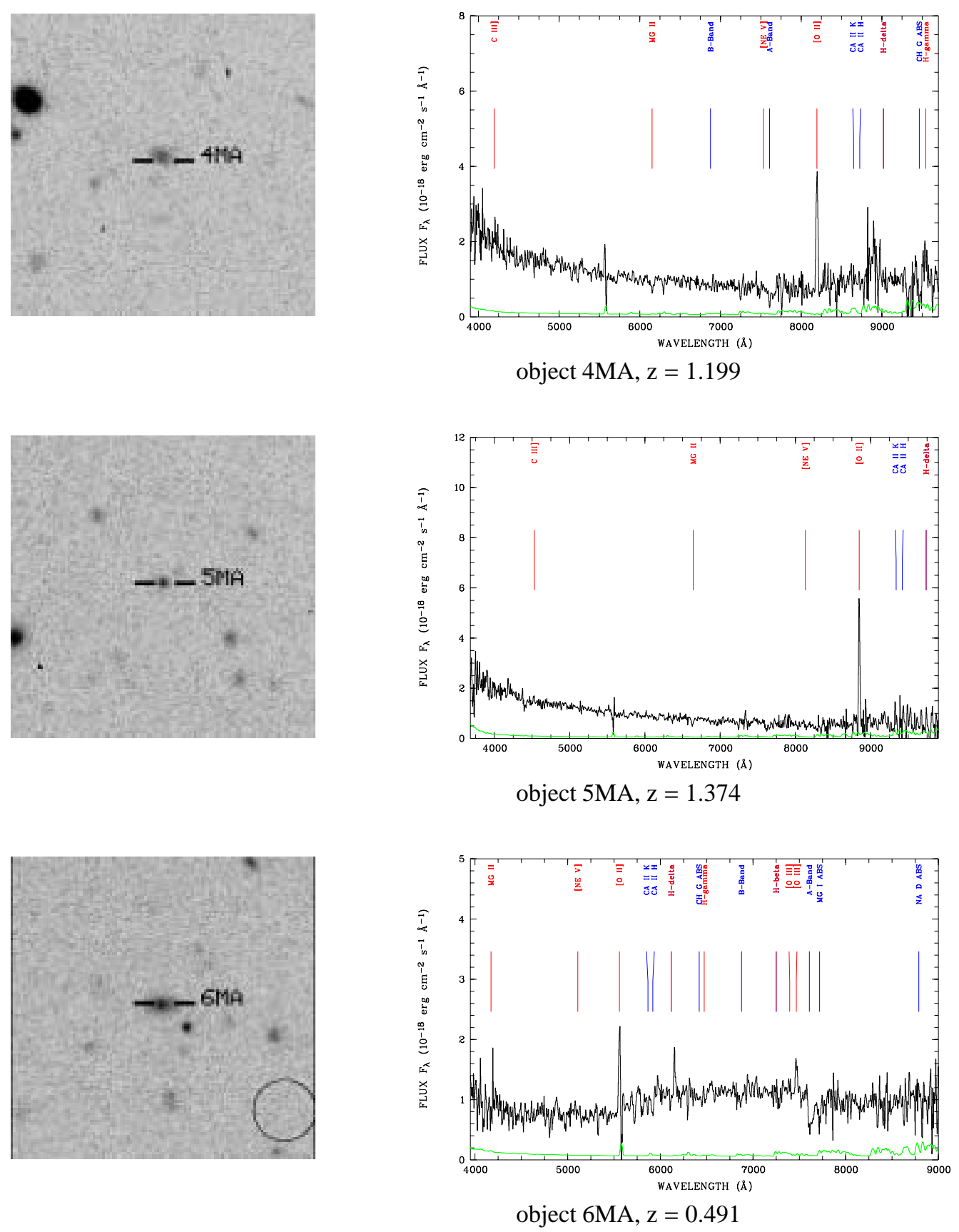

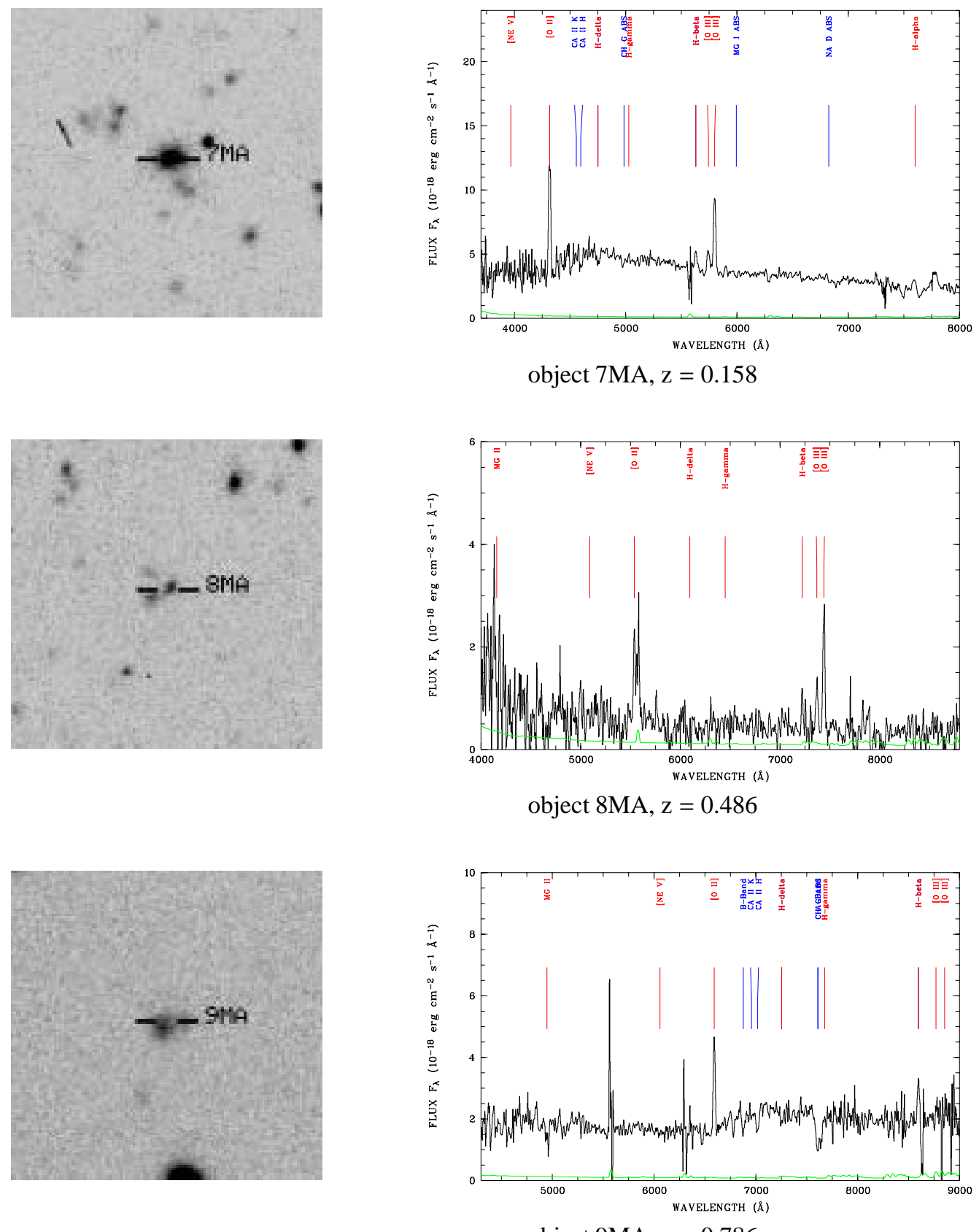

object 9MA, $\mathrm{z}=0.786$
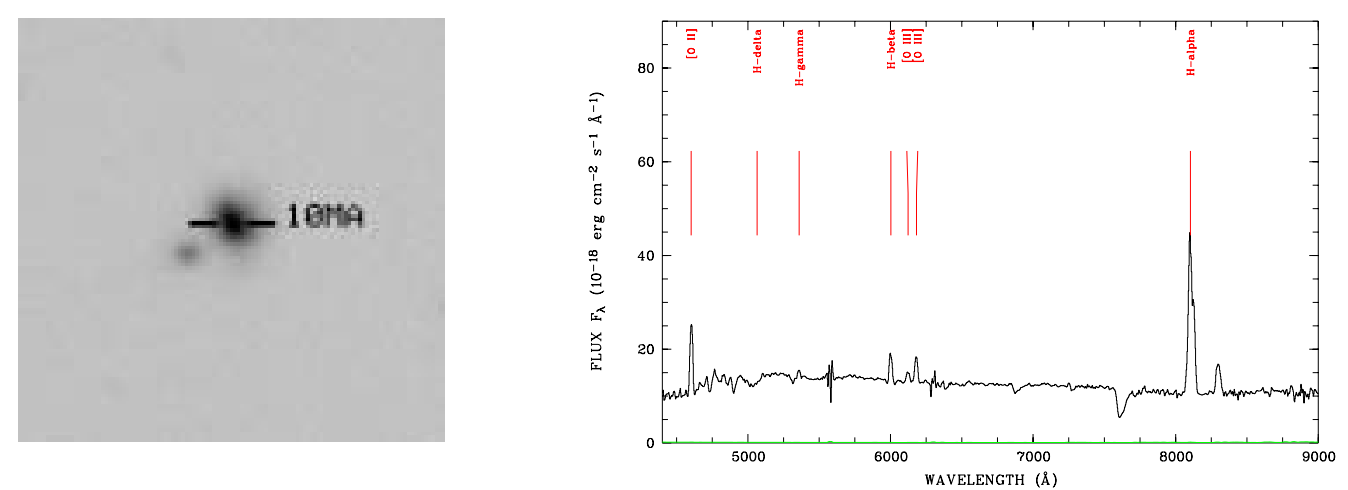

object 10MA, $\mathrm{z}=0.235$ 
M. Krumpe et al.: The XMM-Newton survey in the Marano field. I., Online Material $p 71$
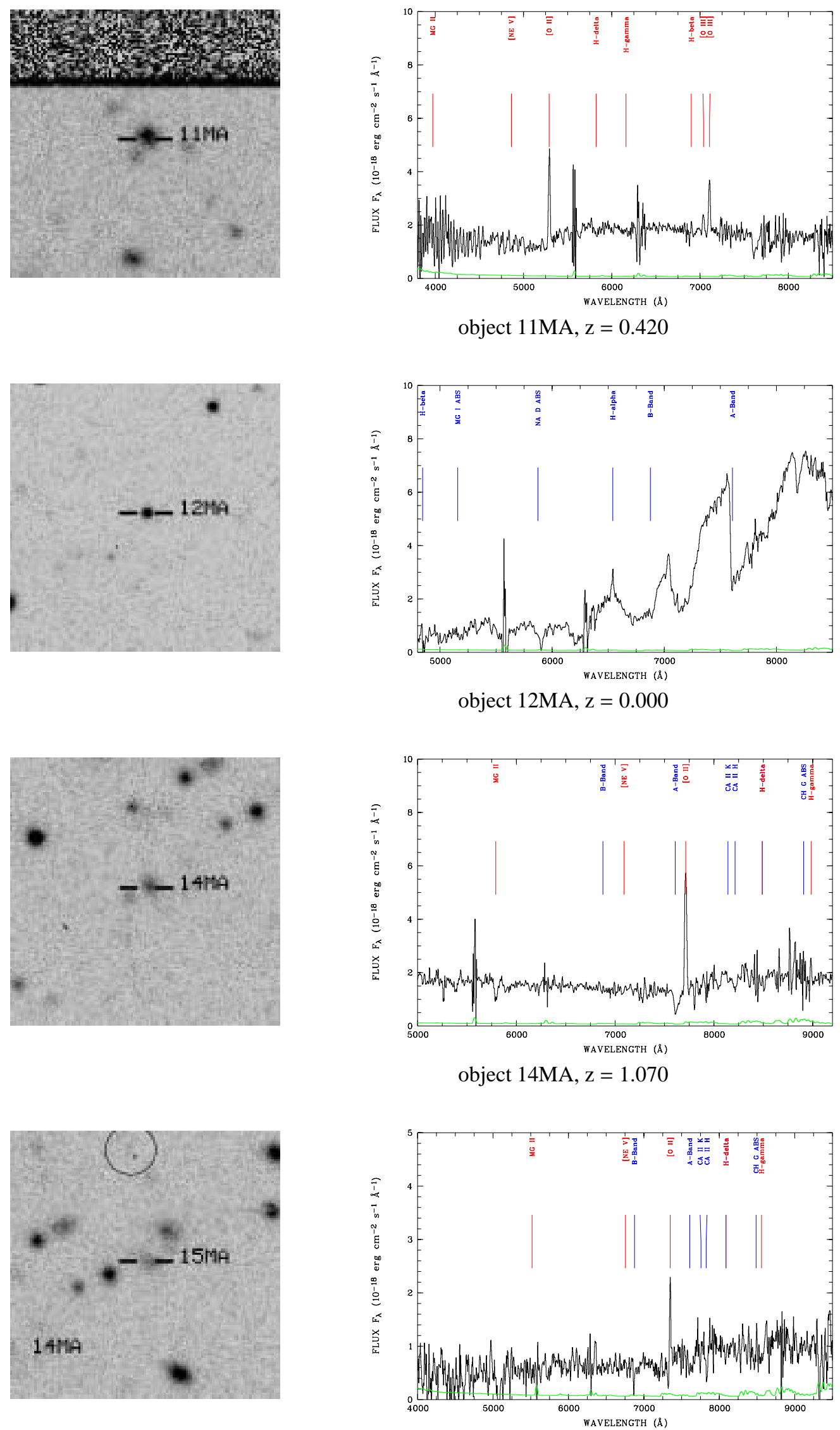

object $15 \mathrm{MA}, \mathrm{z}=0.972$ 

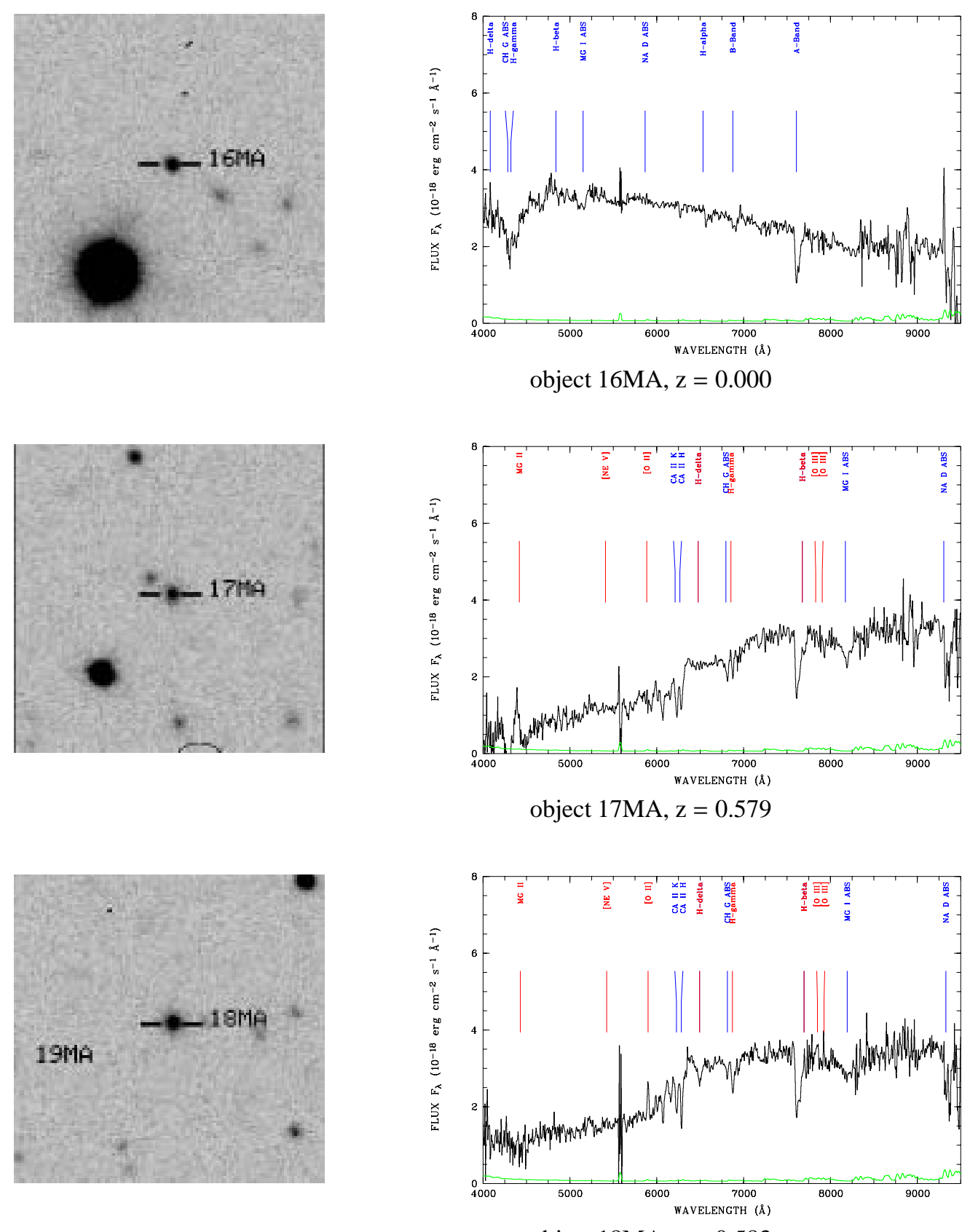

object 18MA, $\mathrm{z}=0.583$
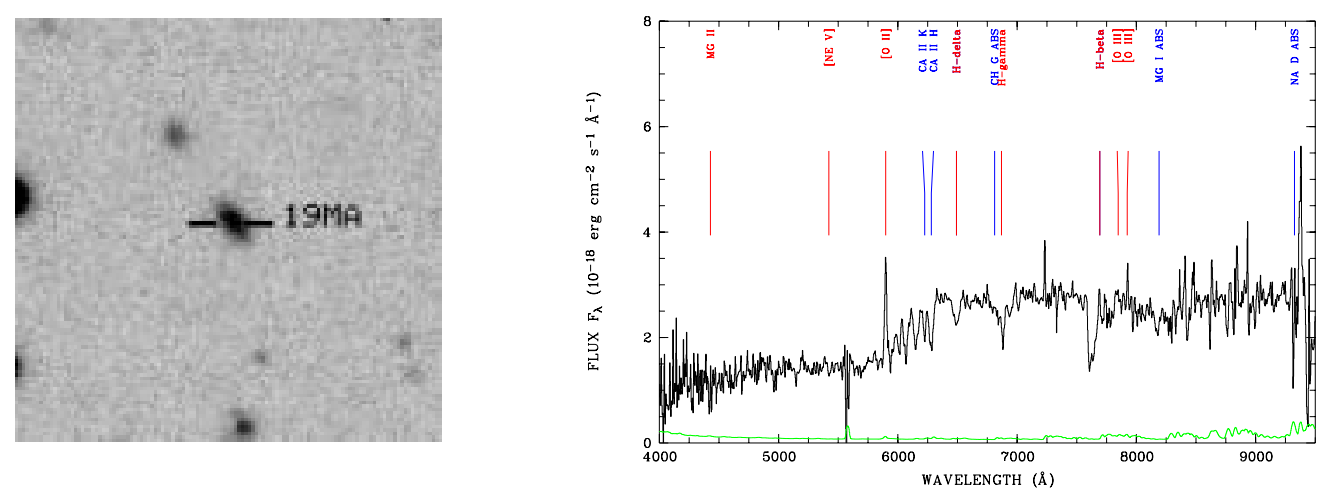

object 19MA, $\mathrm{z}=0.583$ 

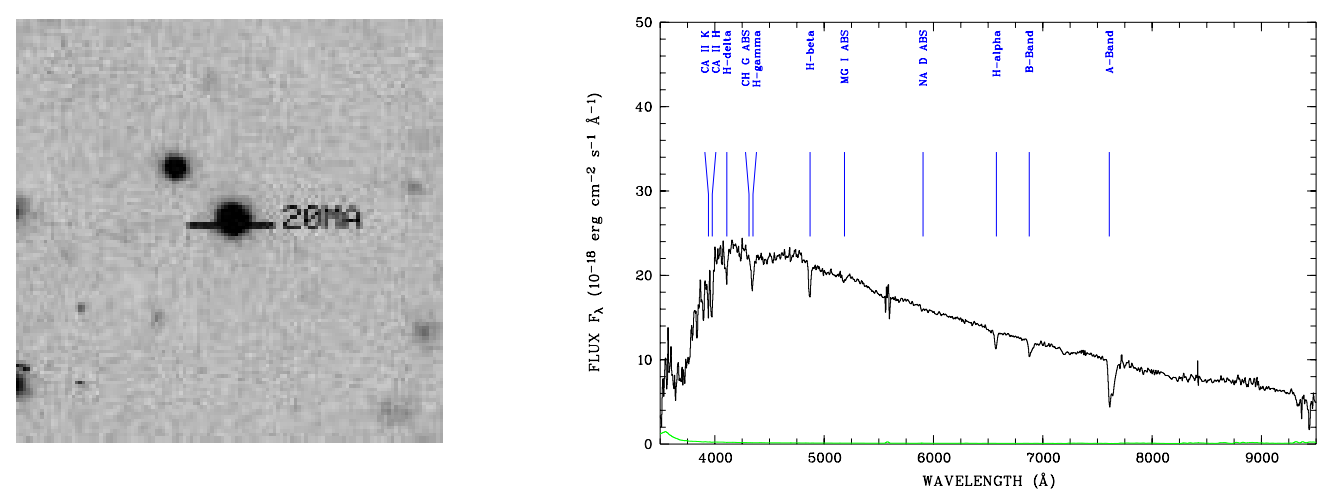

object 20MA, $\mathrm{z}=0.000$
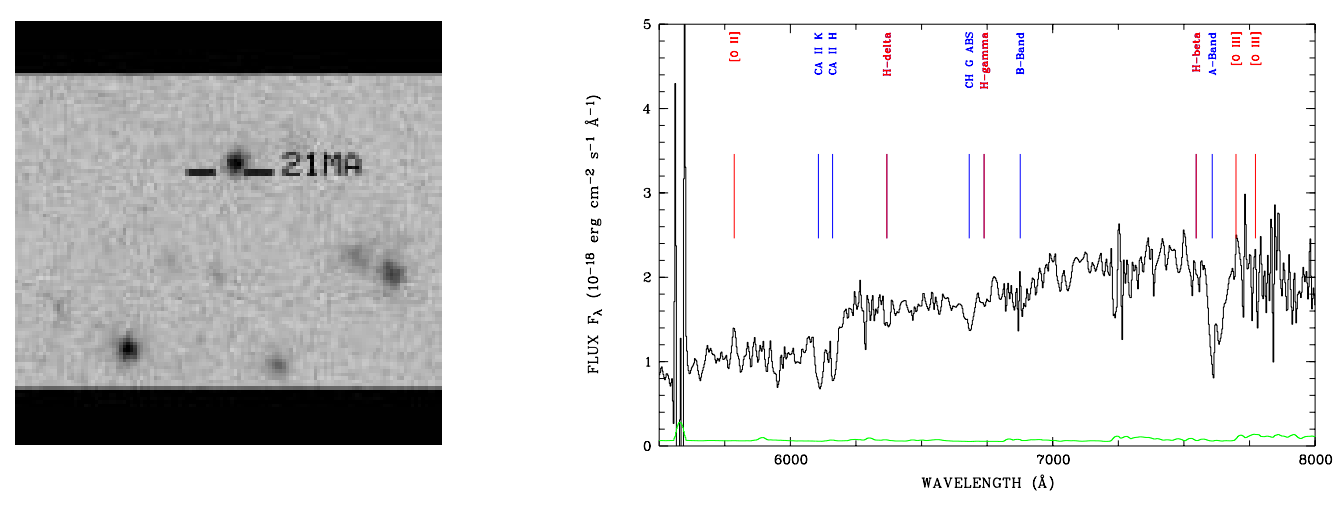

object 21MA, $\mathrm{z}=0.552$
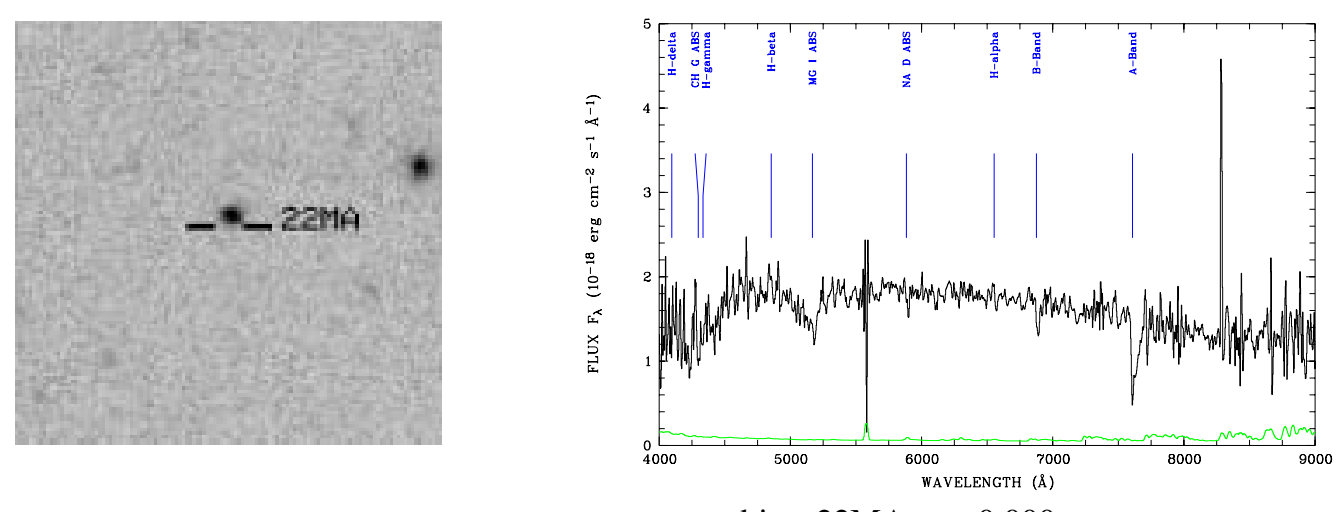

object 22MA, $\mathrm{z}=0.000$
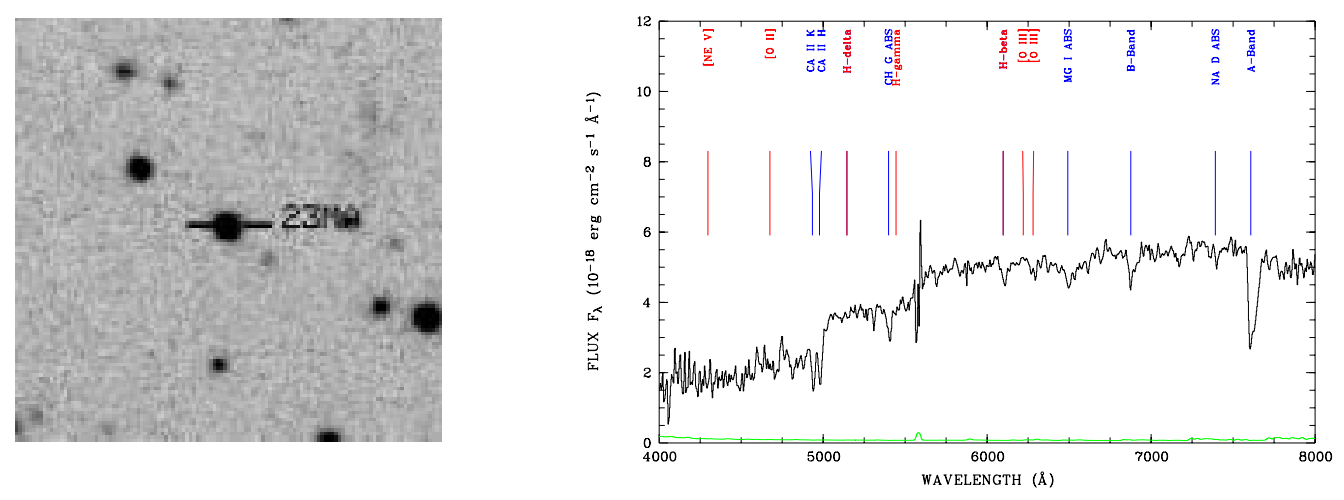

object 23MA, $\mathrm{z}=0.254$ 

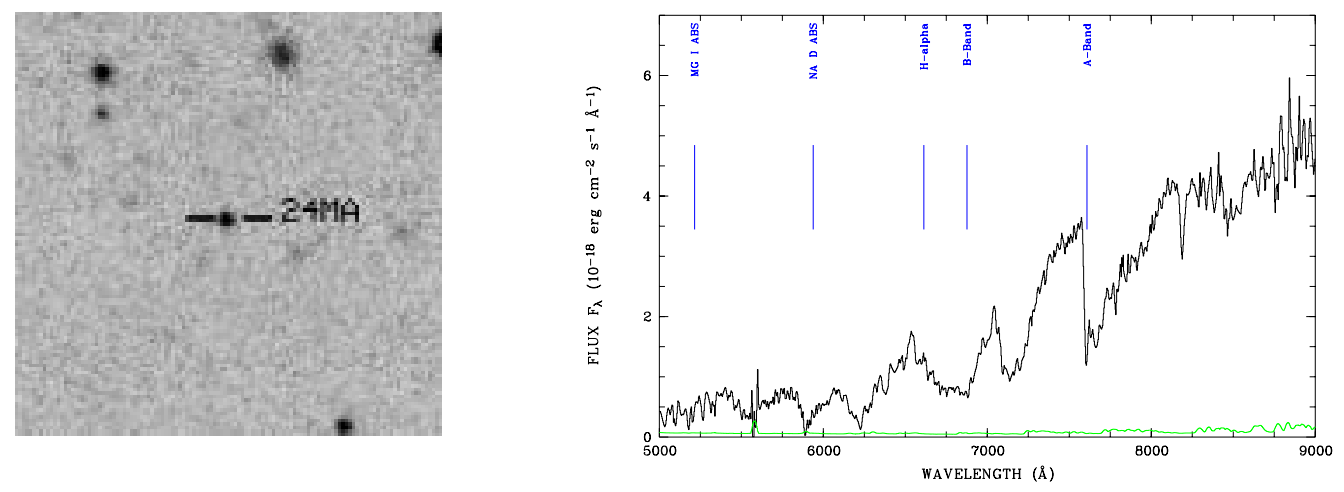

object 24MA, $\mathrm{z}=0.000$
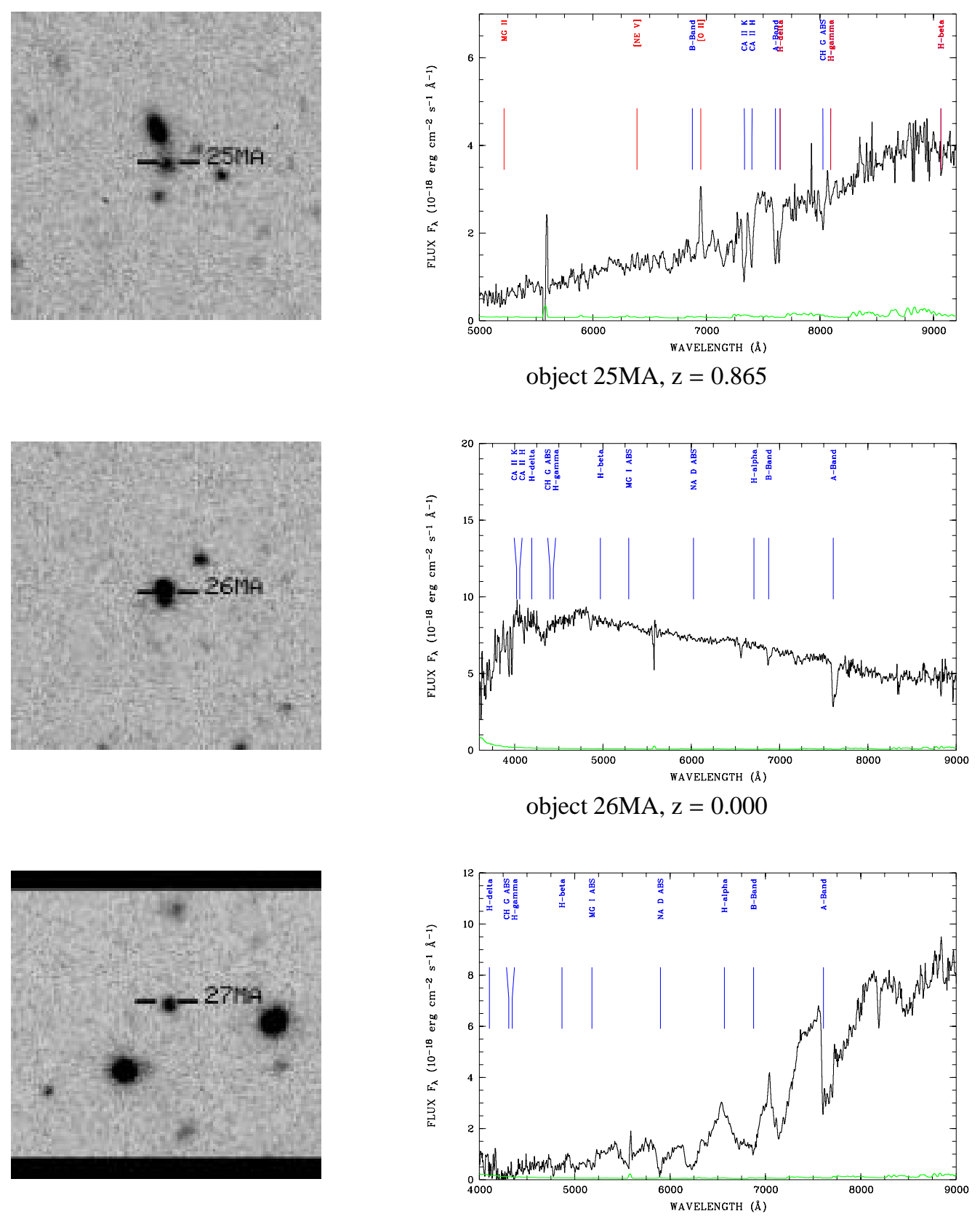

object 27MA, $\mathrm{z}=0.000$ 

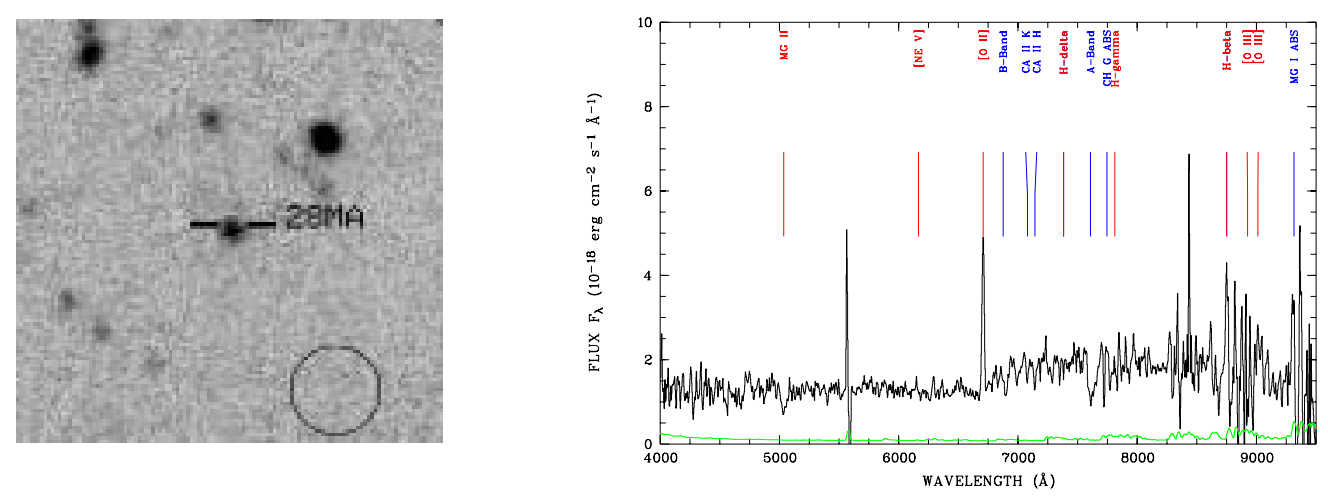

object 28MA, $\mathrm{z}=0.800$
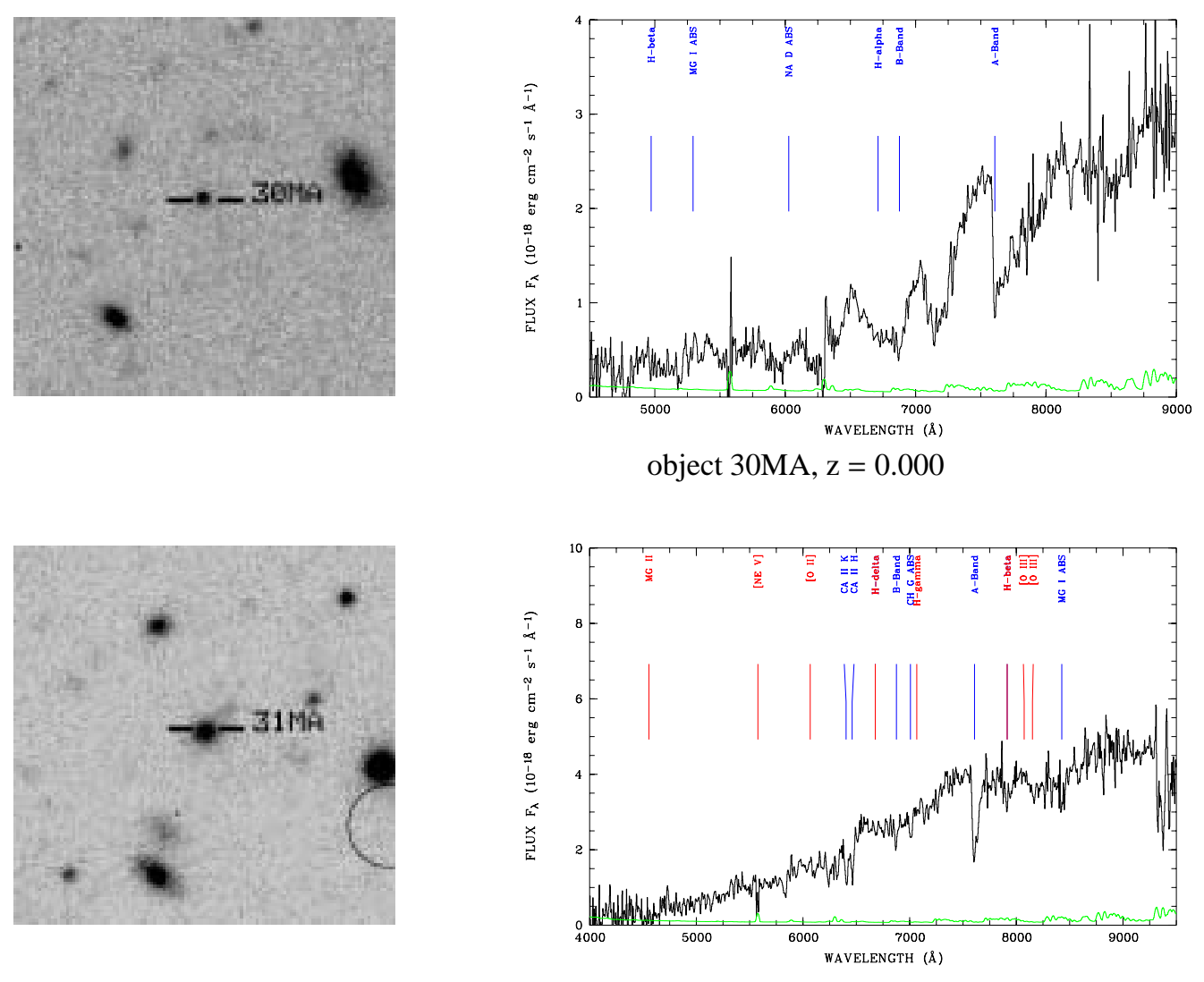

object $31 \mathrm{MA}, \mathrm{z}=0.628$
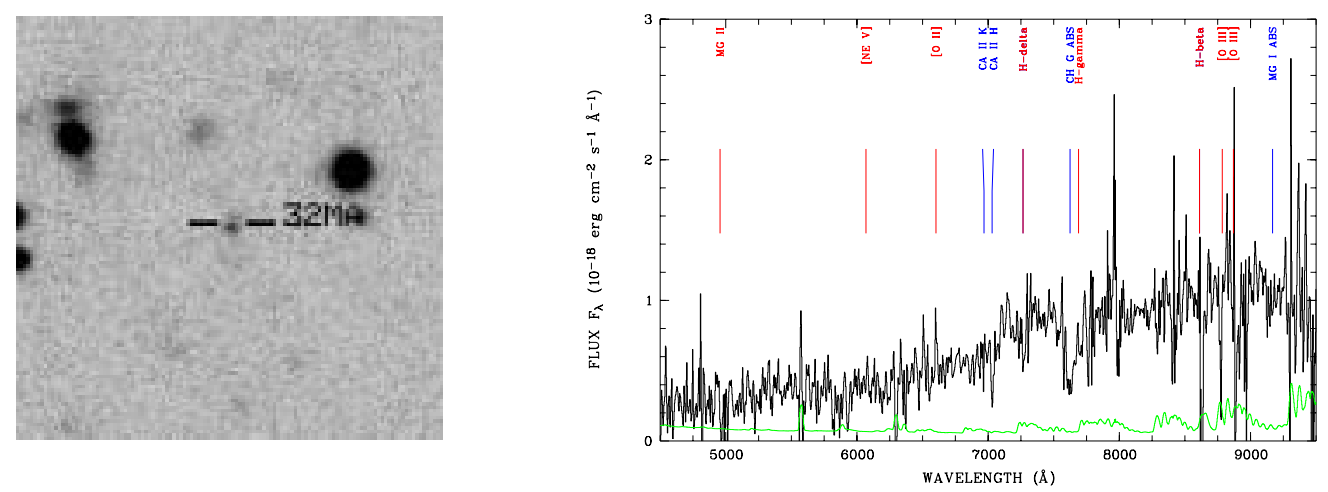

object 32MA, $\mathrm{z}=0.771$ : 

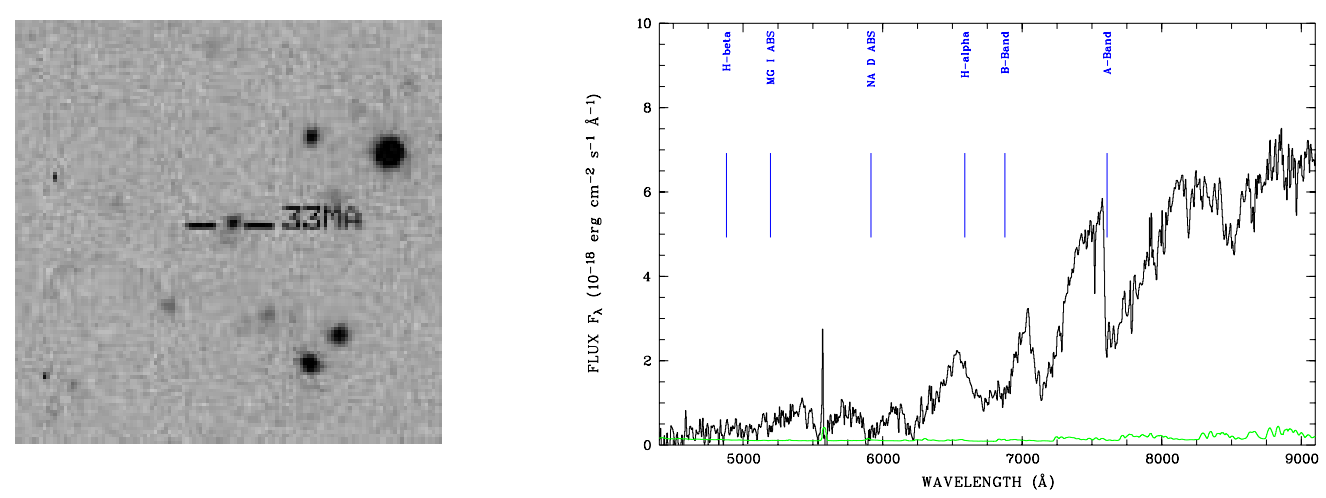

object 33MA, $\mathrm{z}=0.000$
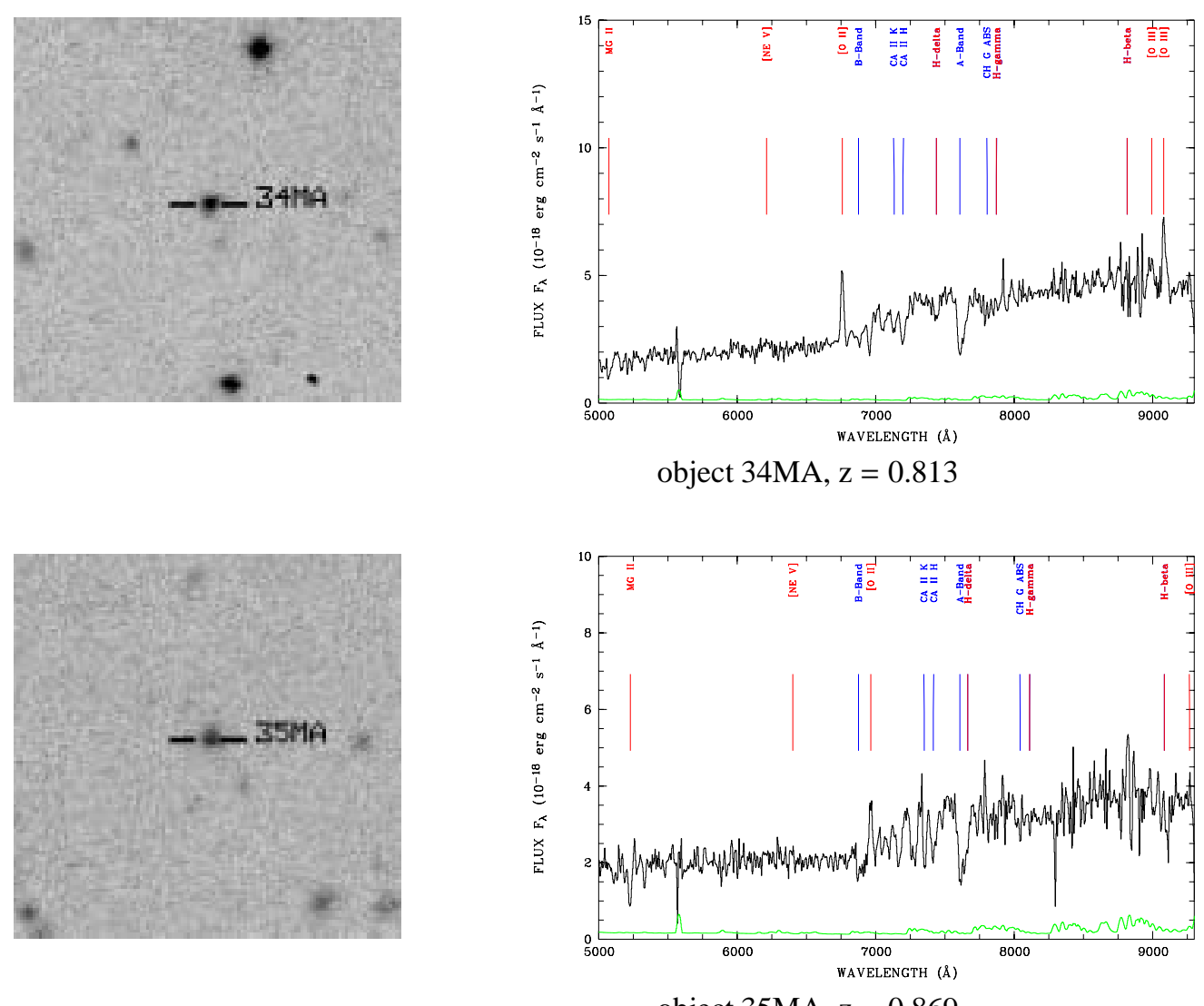

object 35MA, $\mathrm{z}=0.869$
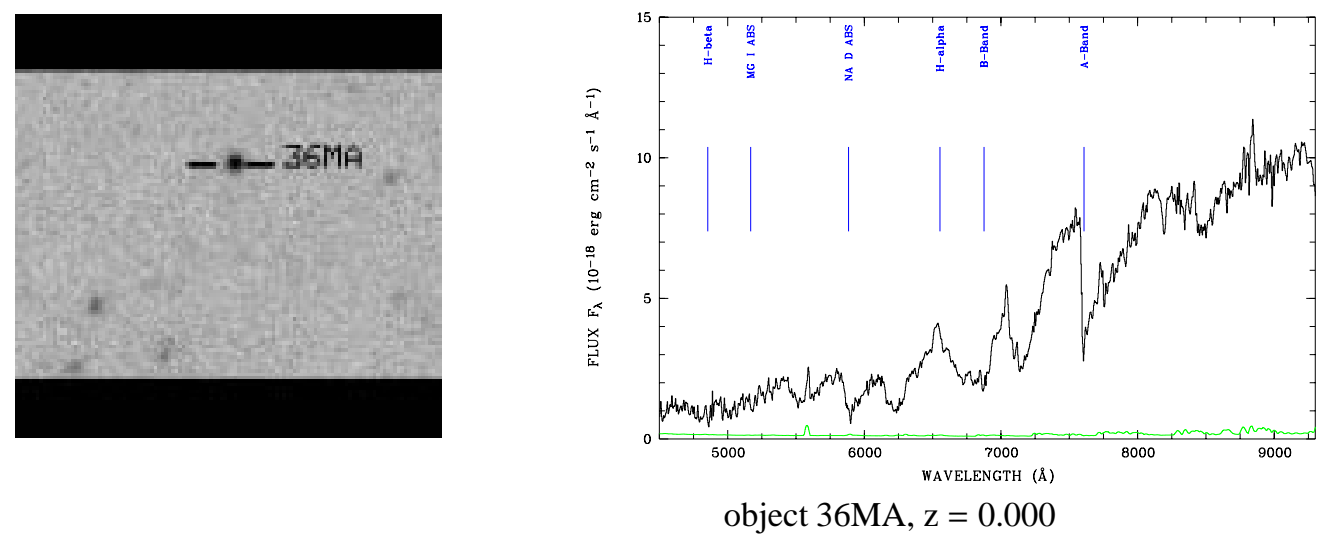

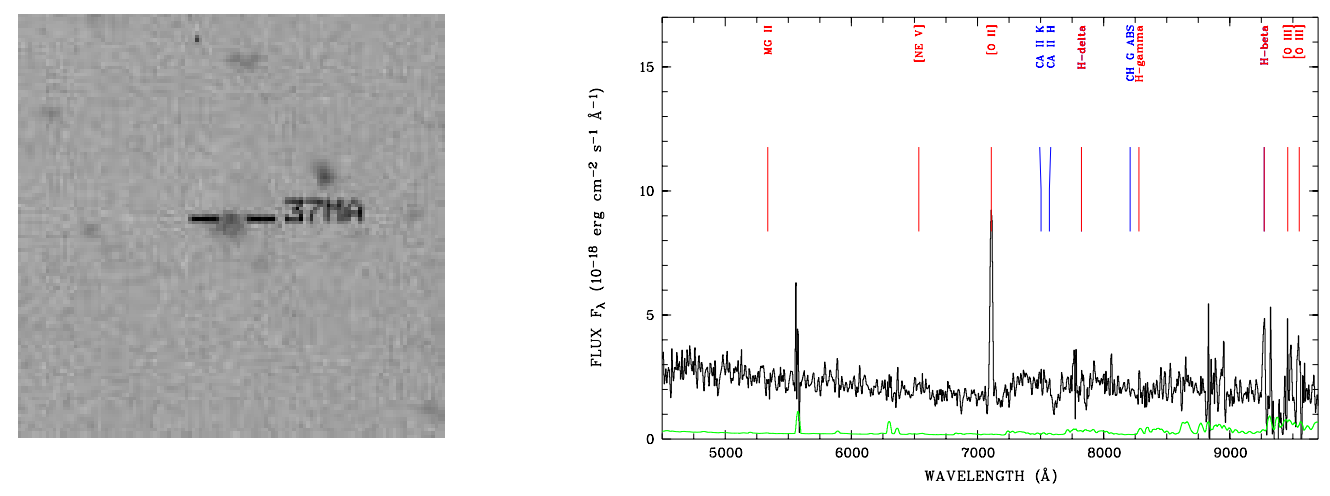

object 37MA, $\mathrm{z}=0.907$
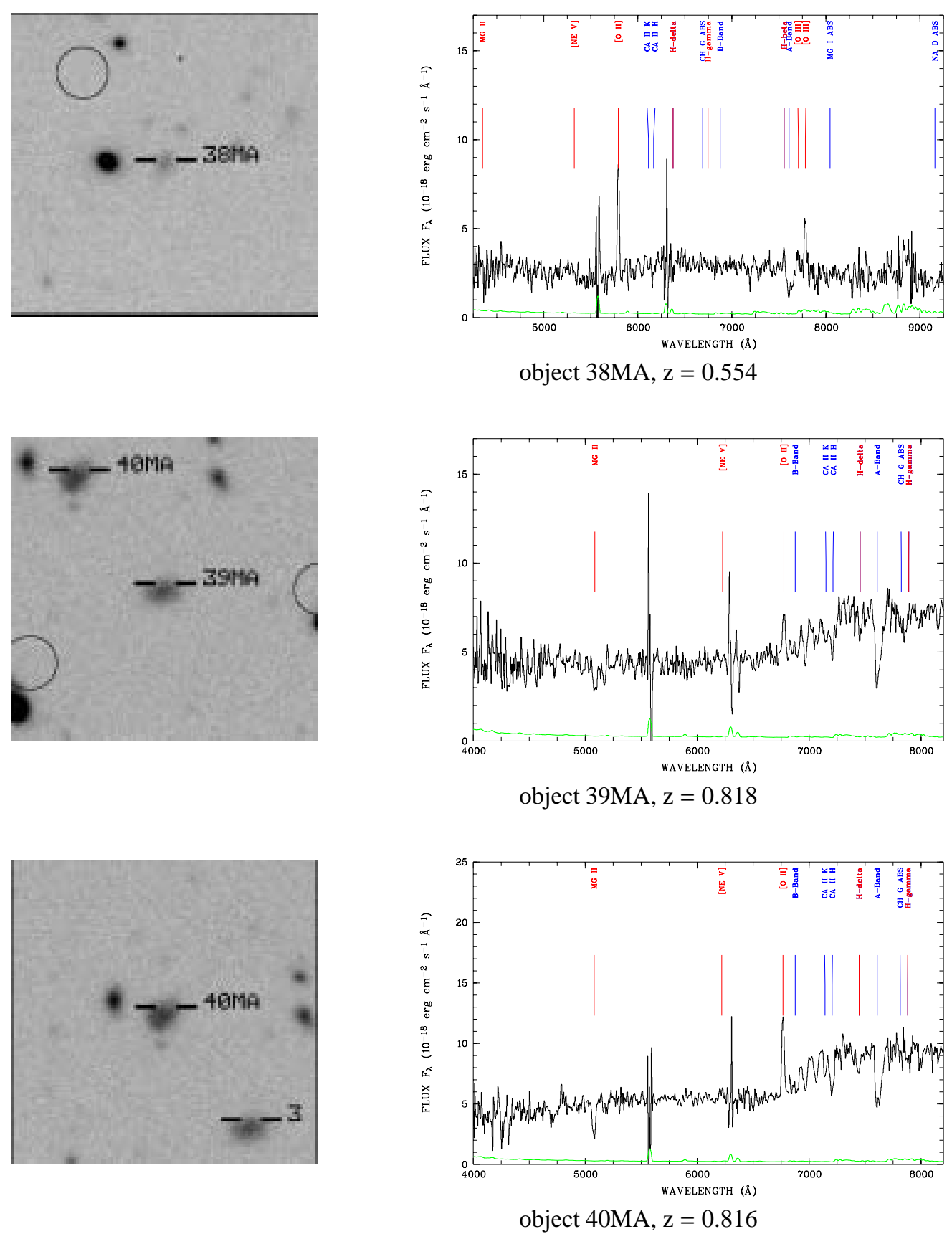
M. Krumpe et al.: The XMM-Newton survey in the Marano field. I., Online Material $p 78$
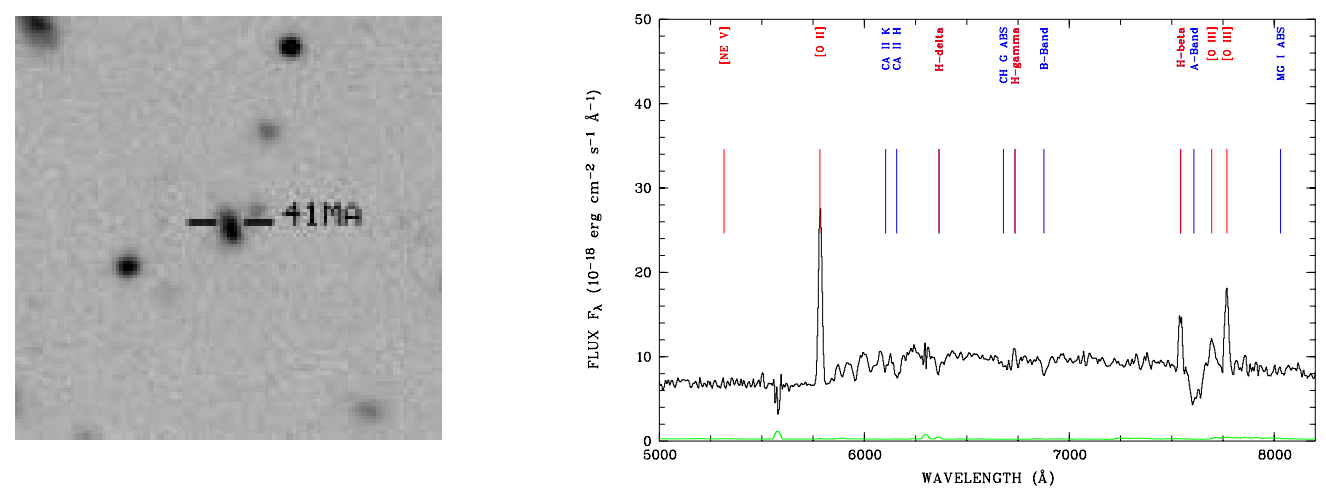

object 41MA, $\mathrm{z}=0.552$
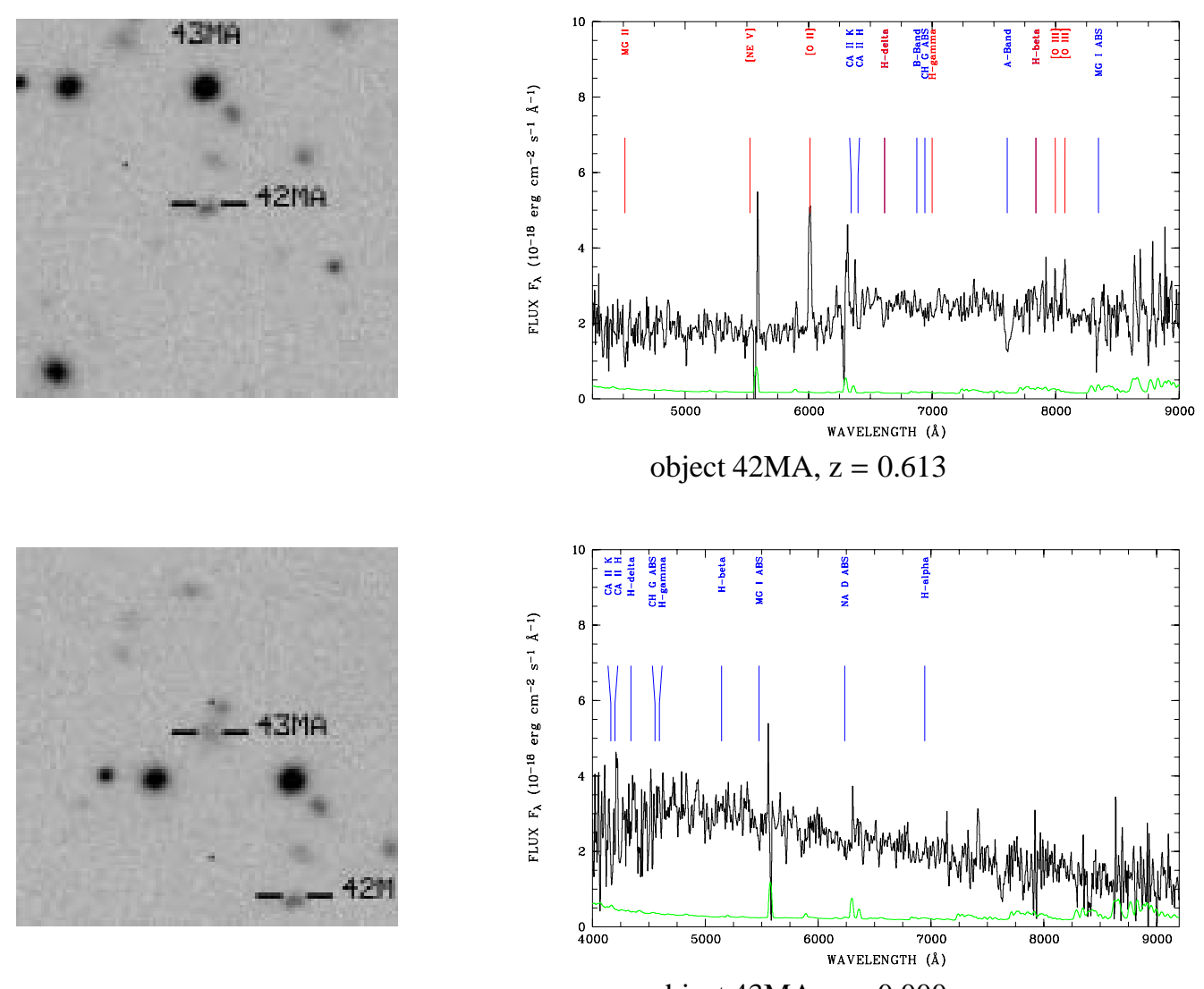

object 43MA, $\mathrm{z}=0.000$
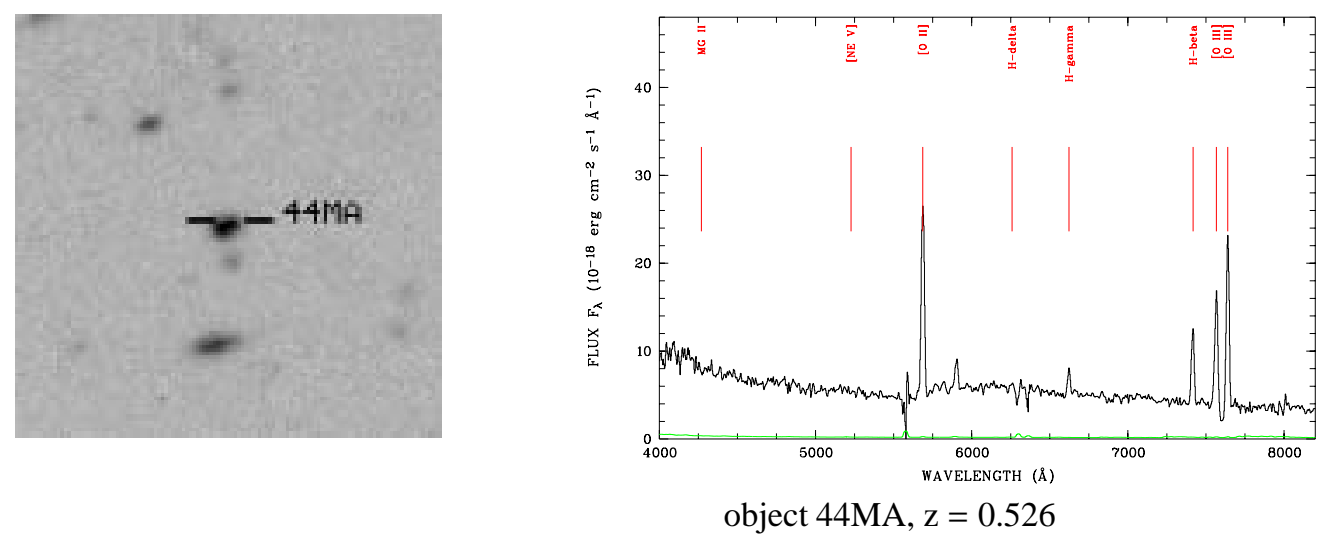

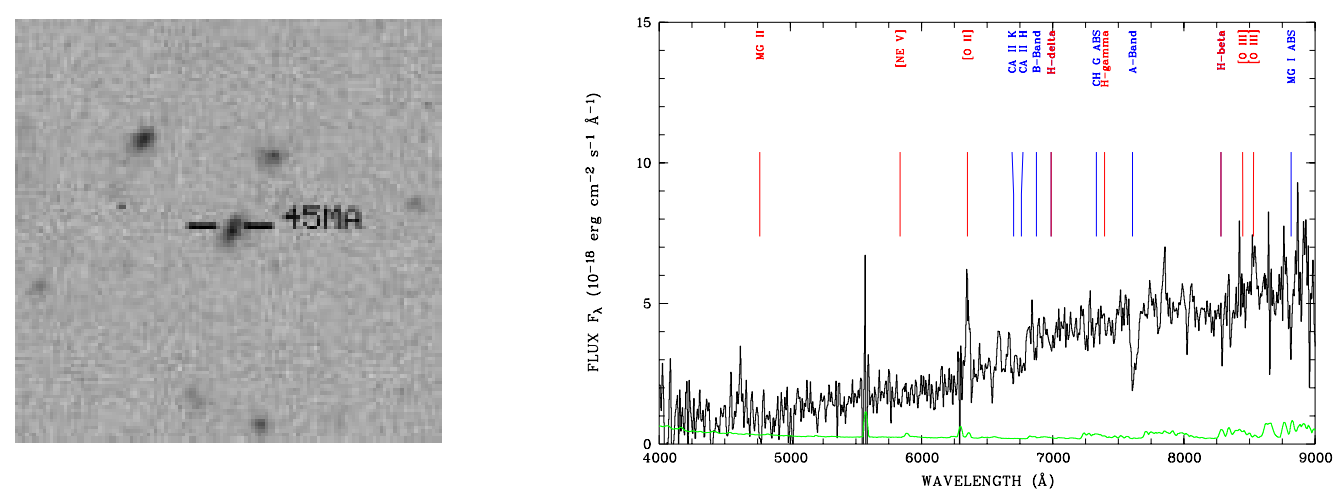

object 45MA, $\mathrm{z}=0.704$
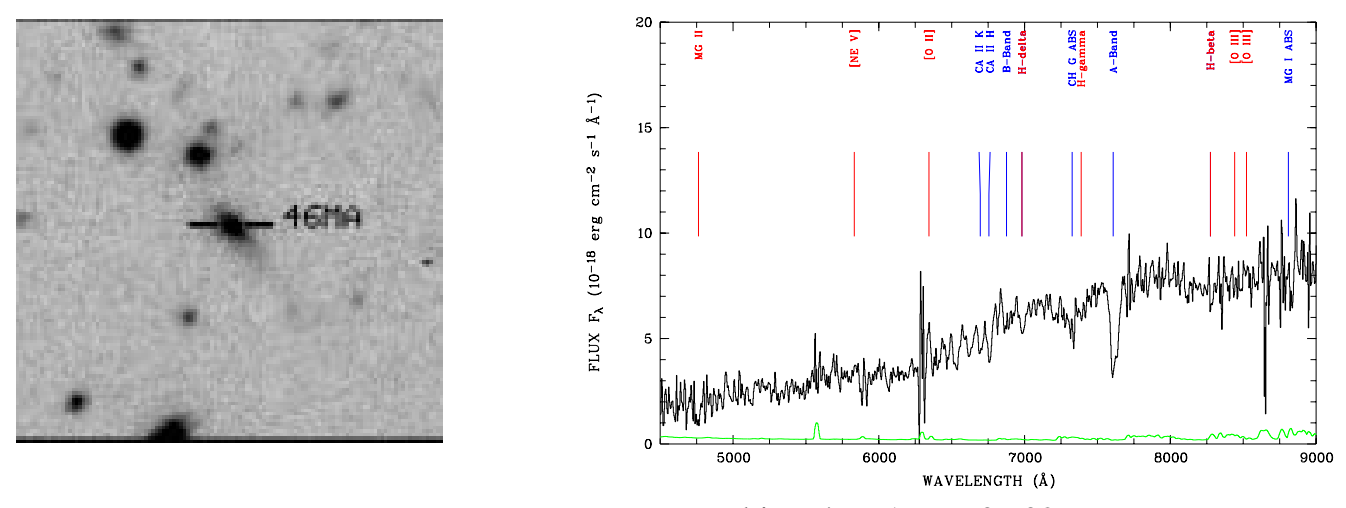

object 46MA, $\mathrm{z}=0.702$
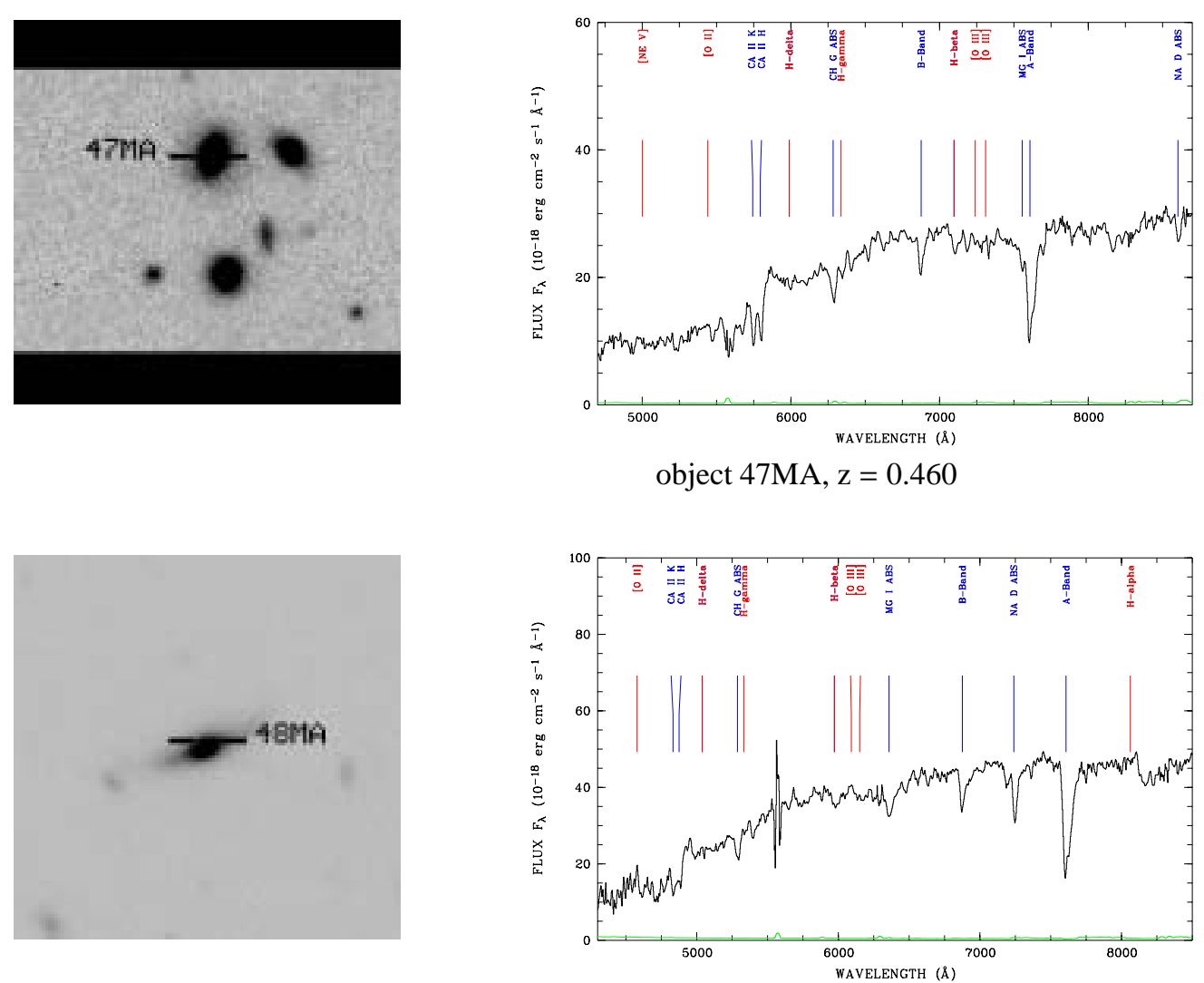

object 48MA, $\mathrm{z}=0.228$ 

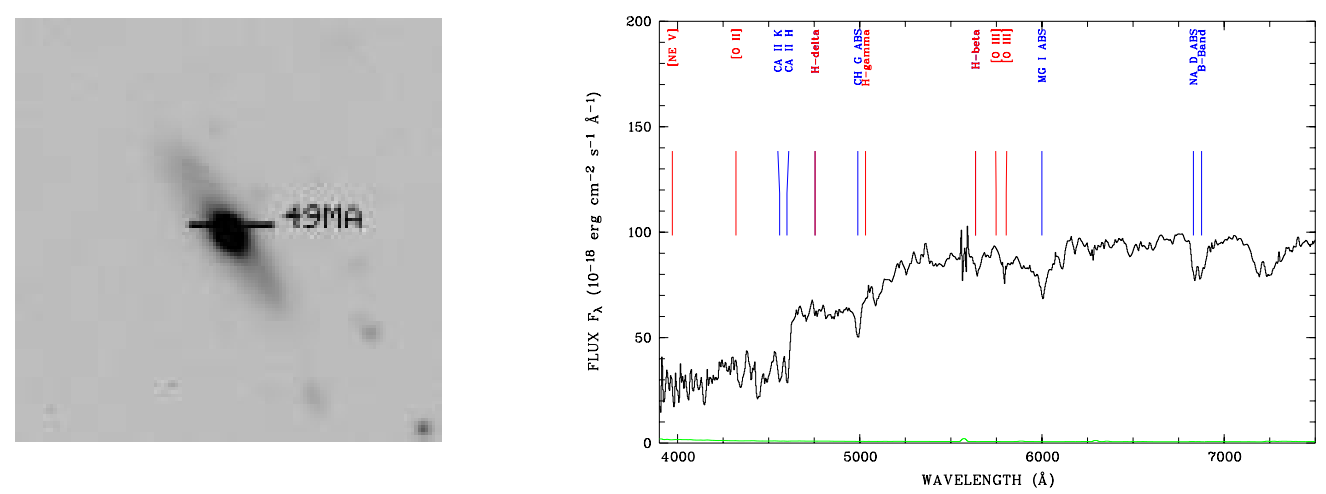

object 49MA, $\mathrm{z}=0.159$
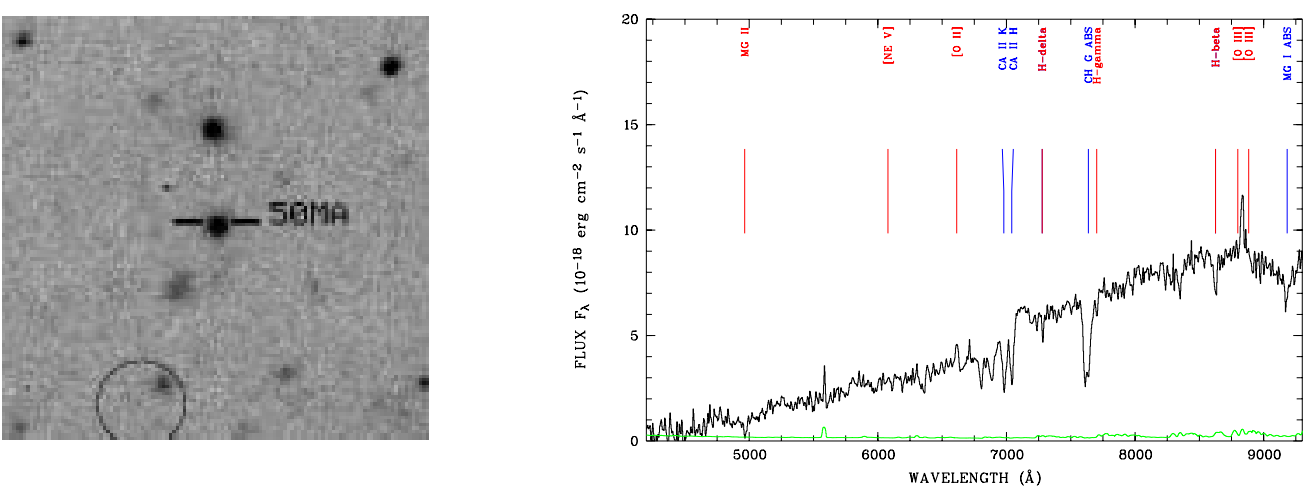

object 50MA, $\mathrm{z}=0.774$
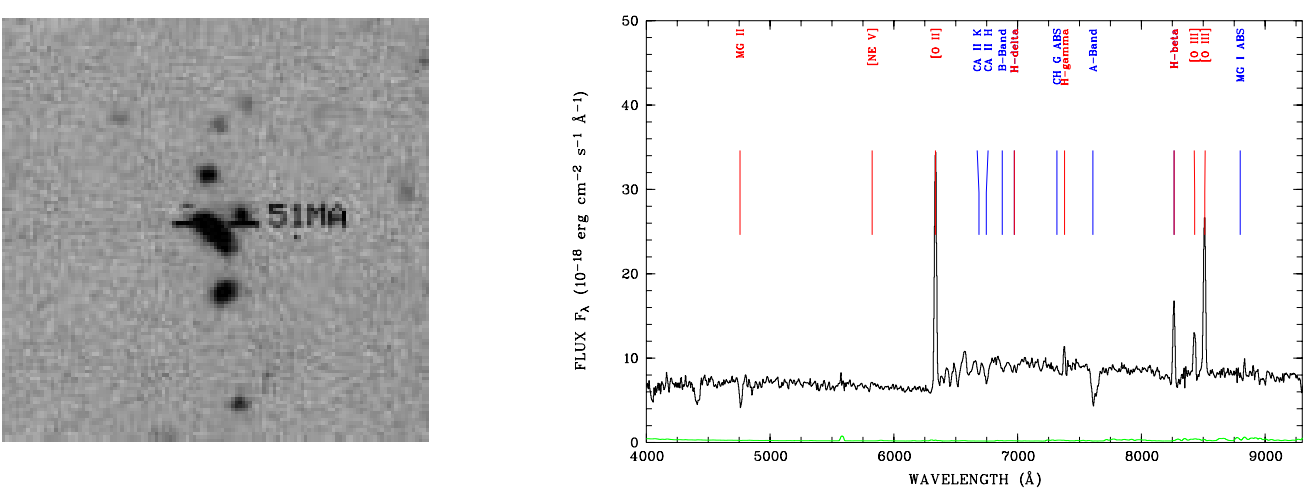

object 51MA, $\mathrm{z}=0.700$
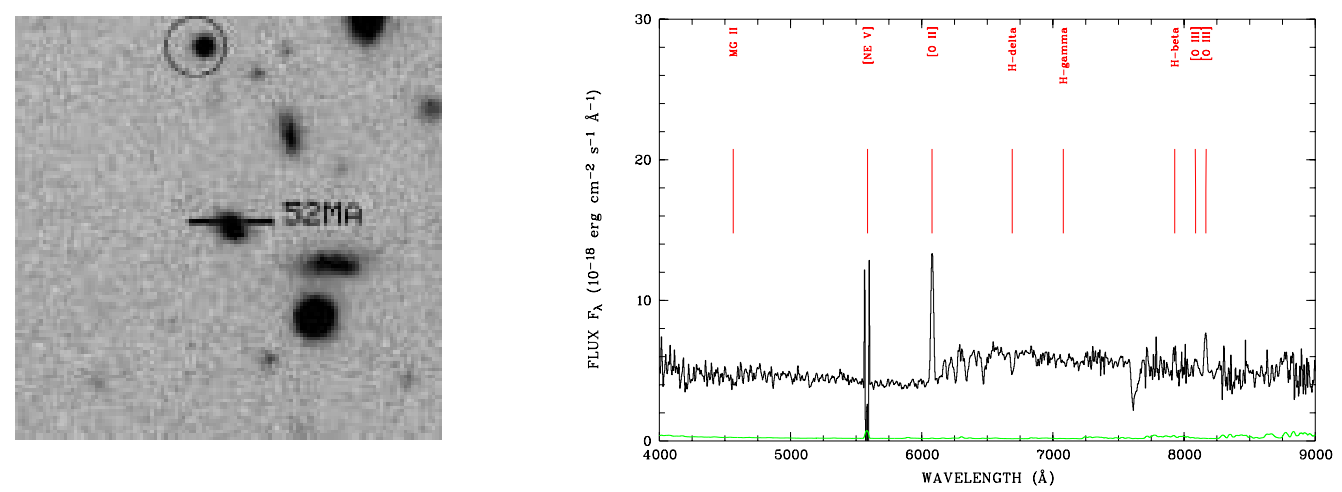

object 52MA, $\mathrm{z}=0.631$ 

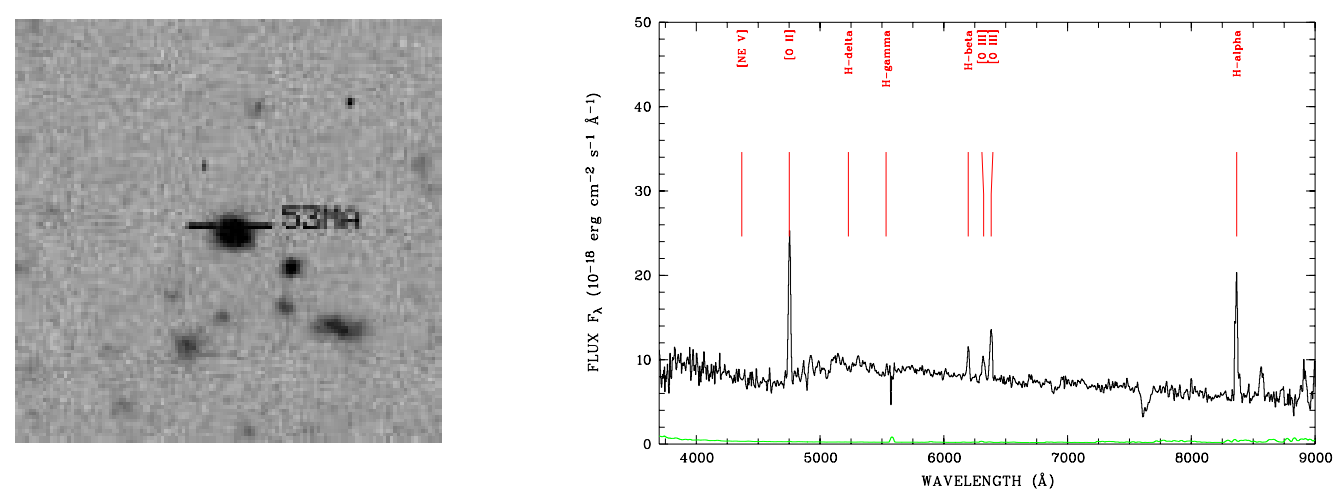

object 53MA, $\mathrm{z}=0.275$
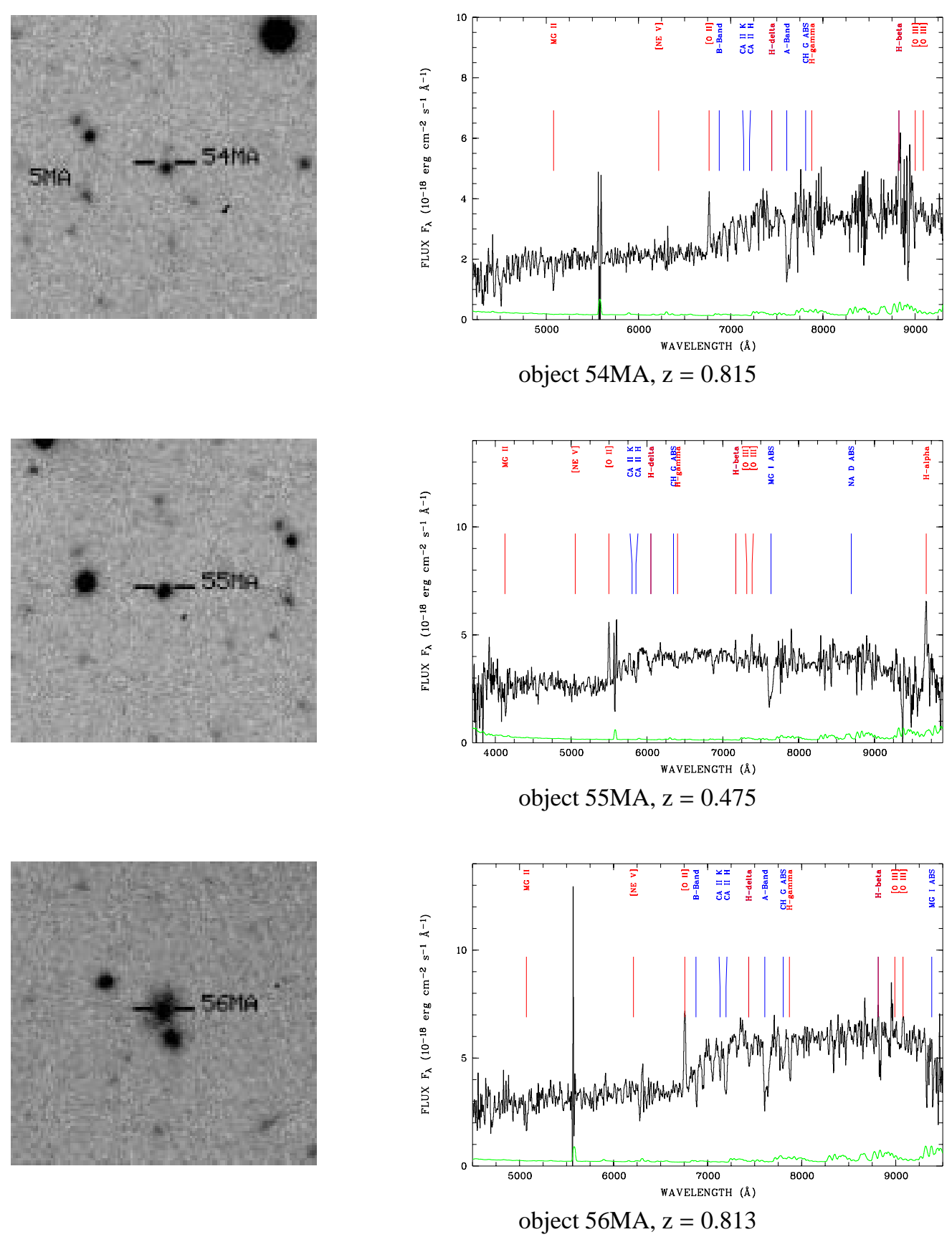

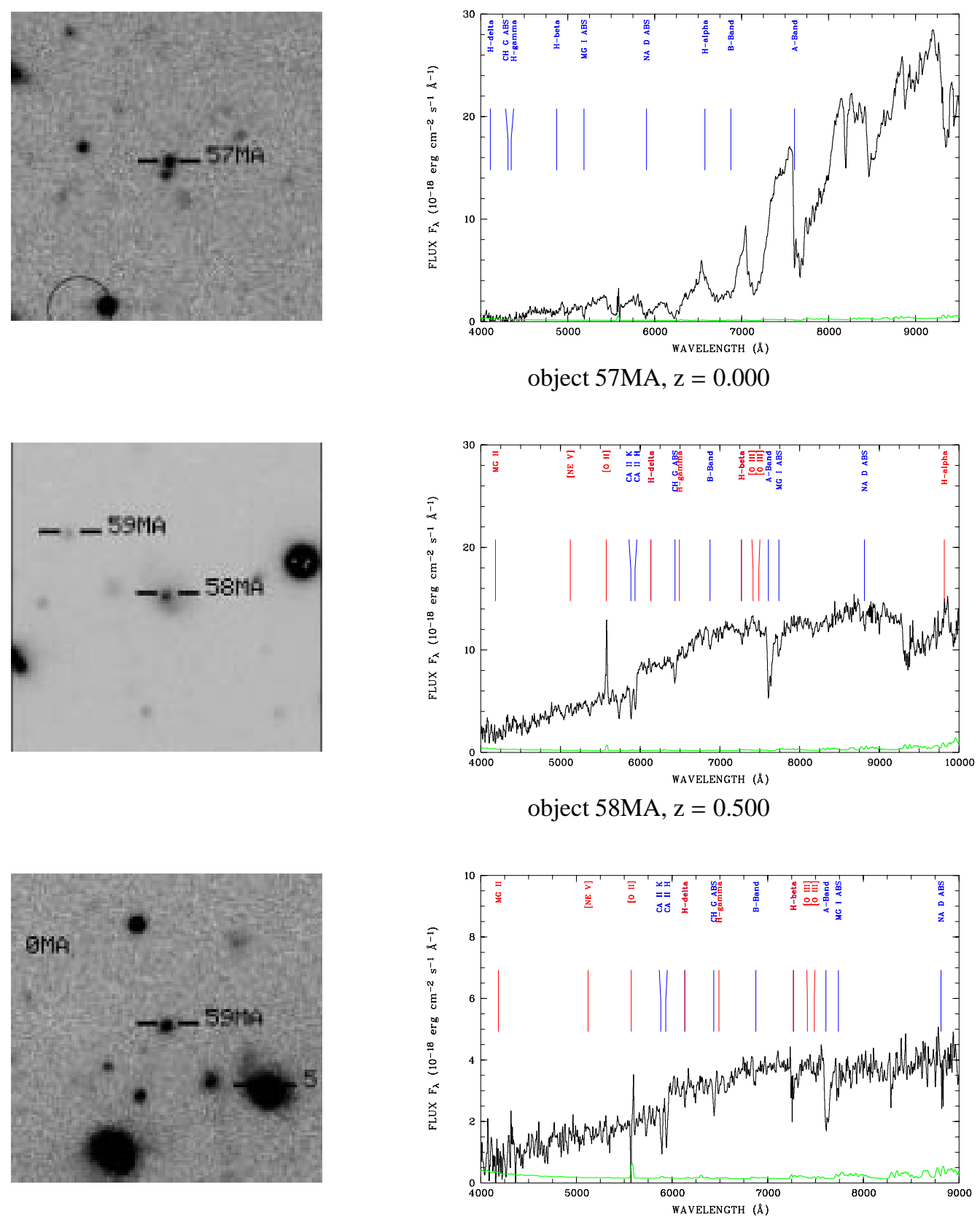

object 59MA, z $=0.495$
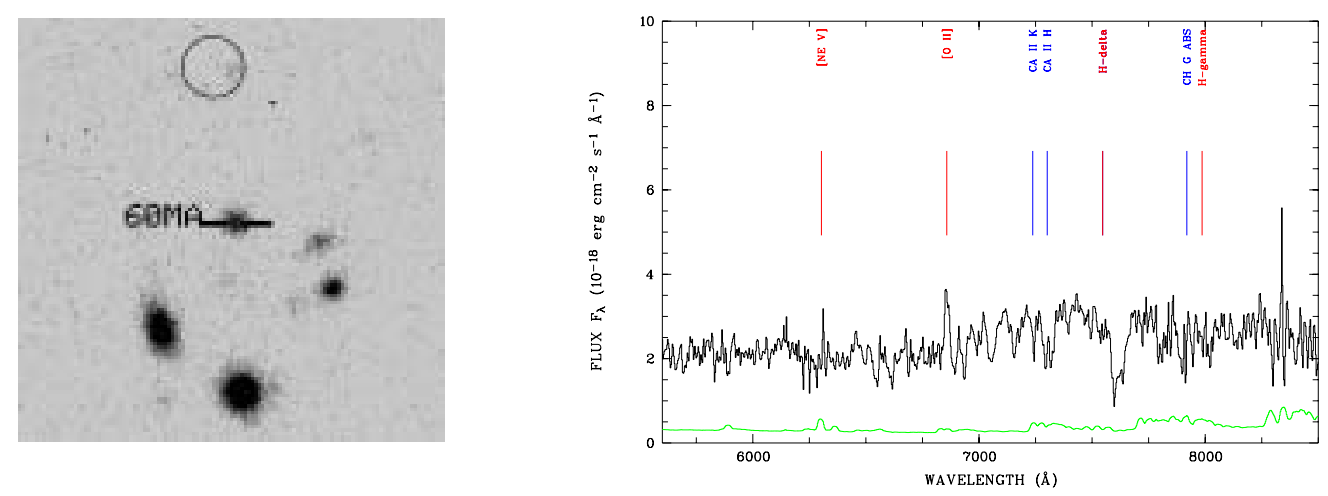

object 60MA, $\mathrm{z}=0.840$ 
M. Krumpe et al.: The XMM-Newton survey in the Marano field. I., Online Material $p 83$
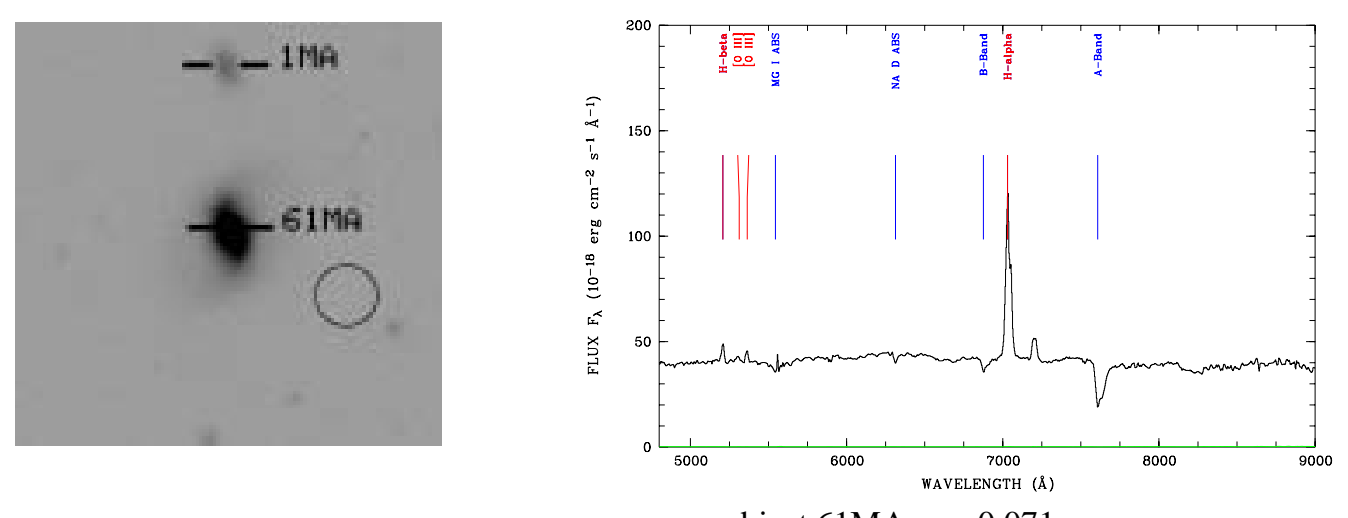

object 61MA, $\mathrm{z}=0.071$
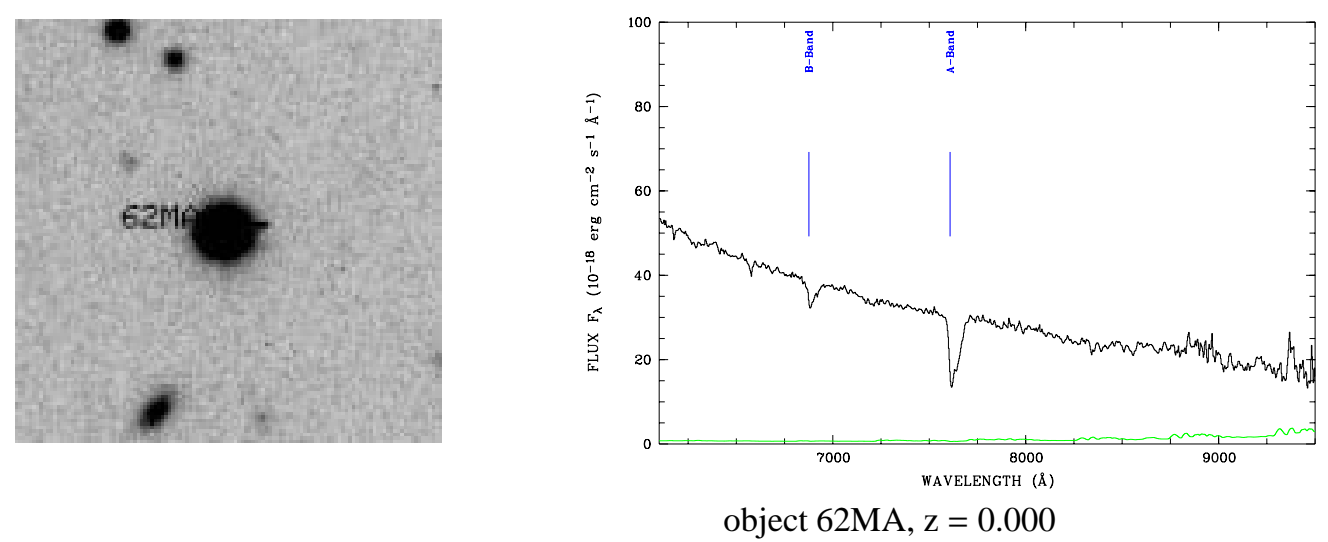